\title{
Aus der Geschichte der Biologischen Anstalt Helgoland (BAH) ab 1945
}

von

Erik Hagmeier

Biologische Anstalt Helgoland (Meeresstation Helgoland),

Postfach 180, D-27483 Helgoland 
Titelfoto:

"100 Jahre Biologische Anstalt Helgoland“, gemalt von Peter Wirsing (Glückstadt). Bild im Besitz der BAH.

Alle Rechte, insbesondere die des Nachdrucks, der Übersetzung und der Verwendung von Abbildungen, bleiben vorbehalten.

ISSN 0174-3597 


\section{EINFÜHRUNG}

\section{Prof. Dr. Wilhelm Nultsch, Direktor der Biologischen Anstalt Helgoland}

Im September 1992 feierte die Biologische Anstalt Helgoland ihr 100jähriges Jubiläum mit einem internationalem Festsymposium mit dem Titel "The Challenge to Marine Biology in a Changing World" unter dem damaligen Direktor Prof. Dr. Wilfried Gunkel. Dieser Geburtstag war zugleich der Anlaß, eine Geschichte der Biologischen Anstalt Helgoland herauszugeben. Frau Dr. Petra Werner übernahm die Bearbeitung des ersten Teiles, der den Zeitraum von der Gründung der Königlich Preußischen Biologischen Anstalt Helgoland bis zum Jahr 1945 abdeckt (Helgoländer Meeresuntersuchungen, Vol. 47 [Suppl.], 1-182, 1993).

Heute liegt nun der Fortsetzungsteil vor, der von Dr. Erik Hagmeier, dem Sohn des ehemaligen Direktors (von 1933 bis 1953) der Biologischen Anstalt Helgoland, verfaßt wurde. Ihm gebührt unser aller Dank dafür, daß er sich dieser mühevollen Aufgabe unterzogen hat. Dies ist um so erfreulicher, als er nicht nur Autor, sondern für viele Ereignisse auch Zeitzeuge ist. Daß infolgedessen auch persönliche Erlebnisse und subjektive Auffassungen in den Text eingeflossen sind, werte ich eher als Vorteil, liest er sich dadurch doch lebendiger und wirklichkeitsnäher. Besonders begrüßenswert ist, daß Herr Hagmeier unter Mitwirkung des Herausgebers Prof. Dr. Klaus Lüning auch zahlreiche Anregungen derzeitiger bzw. ehemaliger Angehöriger der BAH berücksichtigt hat, die an einigen Stellen fehlende persönliche Erfahrungen ergänzen.

Hat die Biologische Anstalt Heigoland als Institut, das vorrangig meeresbiologische Grundlagenforschung betreibt, somit zwei Weltkriege und die mehrfach wechselnde Zuordnung zu verschiedenen Trägern überstanden, so wird in diesem Jahrzehnt ihr Status gravierende Änderungen erfahren. Die Insel Helgoland gehörte seit 1890 zur preußischen Provinz Schleswig-Holstein, die im Jahr 1946 von der britischen Militärregierung zum Land Schleswig-Holstein umgeformt wurde. Nach Auflösung des Staates Preußen durch das Gesetz des Alliierten Kontrollrates vom 25.2.1947 wurde die BAH zunächst der Kultusverwaltung des Landes Schleswig-Holstein unterstellt, ab 1.4. 1948 der Verwaltung für Ernährung, Landwirtschaft und Forsten des Vereinigten Wirtschaftsgebietes und schließlich ab 1.4.1949 dem Bundesernährungsministerium, und zwar in dessen Ressort als Forschungsinstitut an der Bundesanstalt für Fischerei. Bei diesem Trägerwechsel verlor die BAH den Landespartner, der als Träger oder zumindest als mitbeteiligter Träger für Institute der Grundlagenforschung in Deutschland unerläßlich ist. Fast 50 Jahre später wurde dieser Formfehler korrigiert, allerdings nicht mehr mit SchleswigHolstein als Hauptträger (siehe unten). Im Zeitraum 1949-1997 bereitete der Umstand, daß die $\mathrm{BAH}$ sich keineswegs vorrangig mit Fischereiwissenschaft befaßte, sondern überwiegend mit meeresbiologischer Grundlagenforschung, und daß sie darüber hinaus auch noch "Unterricht" in Form der meeresbiologischen Kurse betrieb, der ministeriellen Bürokratie große Probleme.

So wurde die BAH, eben wegen ihrer Tätigkeiten für die Universitäten, 1971 dem Bundesministerium für Bildung und Wissenschaft zugeordnet. Aber bereits 1972 wurde die BAH, wohl weil ihr zweiter Schwerpunkt eben die Forschung war, dem Bundesministerium für Forschung und Technologie (BMFT) unterstellt, das nach Zusammenlegung der beiden Ministerien am 17.11.1994 als Bundesministerium für Bildung, Wissenschaft, Forschung und Technologie (BMBF) firmierte. 
Man sollte meinen, daß damit die Odyssee der BAH bezüglich der Trägerschaft ein Ende gefunden habe. Dies trifft jedoch nicht zu, weil, wie erwähnt, der Landespartner als Träger fehlte. Die BAH war und ist die einzige Bundesanstalt, die überwiegend Grundlagenforschung betreibt und keine hoheitlichen Aufgaben erfüllt. Außerdem hat der Status einer direkt nachgeordneten Behörde auch gewisse Nachteile für ein Grundlagenforschungsinstitut, auf die hier nicht im einzelnen eingegangen werden kann. Aus diesem Grund kam das BMBF (ex BMFT) Anfang der 90er Jahre zu der Entscheidung, die BAH als nachgeordnete Behörde des Ministeriums aufzulösen und ihr eine andere Struktur zu geben. Auf Wunsch des Ministeriums setzte der Wissenschaftsrat eine Kommission ein, die die BAH im März 1994 begutachtete und ihren Erhalt unter Beibehaltung ihres traditionsreichen Namens befürwortete. Hinsichtlich der künftigen Struktur schlug sie vor, die BAH entweder in ein Blaue-Liste-Institut (Finanzierung: $50 \%$ Bund, $50 \%$ Land bzw. Länder) mit Schleswig-Holstein und gegebenenfalls anderen Ländern umzuwandeln oder sie als eigenständiges, zweites Institut unter dem Dach der Großforschungseinrichtung "Stiftung Alfred-Wegener-Institut" (AWI) anzusiedeln, einer Stiftung des öffentlichen Rechts der Freien Hansestadt Bremen. Nachdem die Verhandlungen des BMBF mit den Ländern Hamburg und Schleswig-Holstein nicht zu einem das BMBF befriedigenden Ergebnis geführt haben, wird jetzt ab 1. Januar 1998 der zweite Vorschlag umgesetzt, also die Eingliederung der BAH in das AWI (Finanzierung: $90 \%$ Bund, $8 \%$ Bremen, $1 \%$ Schleswig-Holstein, $1 \%$ Brandenburg). Das Land Bremen hat die Verlegung der BAH-Zentrale von Hamburg nach Bremerhaven zur Voraussetzung für seine Zustimmung gemacht, eine für viele BAH-Mitarbeiter gravierende Entscheidung.

Ob diese Entscheidung weise war, und ob die Eigenständigkeit unserer über 100 Jahre alten Institution unter diesen Umständen erhalten bleibt, wird die Zukunft erweisen. Ich kann dies sowohl im Interesse der BAH als auch der Universitäten und der einschlägigen Forschungsinstitute nur wünschen.

Hamburg, im Dezember 1997

Wilhelm Nultsch 


\section{INHALTSVERZEICHNIS}

1. Das Ende des Zweiten Weltkriegs auf Helgoland $\ldots \ldots \ldots \ldots \ldots \ldots \ldots \ldots$

2. Der Neuanfang auf dem Festland (Wedel, Altona, Cuxhaven) $\ldots \ldots \ldots \ldots \ldots 8$

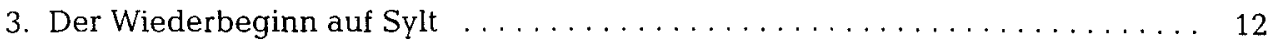

4. Zur Finanzierung und Trägerschaft der BAH $\ldots \ldots \ldots \ldots \ldots \ldots \ldots \ldots \ldots \ldots$

5. Die Wiedergewinnung von Helgoland $\ldots \ldots \ldots \ldots \ldots \ldots \ldots \ldots \ldots \ldots \ldots \ldots$

6. Professor Bückmann und die "Kopfstation" der BAH ............. 26

7. Mitarbeit der BAH in der Deutschen Wissenschaftlichen Kommission für

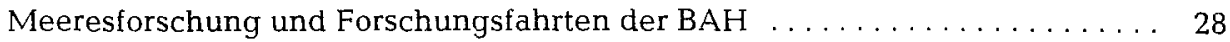

8. Wiederaufbau auf Helgoland, Mitarbeiter, Gäste und Kurse . . . . . . . . . . 32

9. Koordination der Meeresforschung im Nachkriegs-Deutschland . . . . . . 36

10. Professor Kinne und die BAH im Jahr $1962 \ldots \ldots \ldots \ldots \ldots \ldots \ldots \ldots \ldots$

11. Das Schwerpunktprogramm ab $1963 \ldots \ldots \ldots \ldots \ldots \ldots \ldots \ldots \ldots \ldots \ldots$

12. Symposien und Arbeitstreffen an der BAH bis $1983 \ldots \ldots \ldots \ldots \ldots \ldots \ldots .44$

13. Schiffe und Forschungsfahrten $1962-1983 \ldots \ldots \ldots \ldots \ldots \ldots \ldots \ldots$

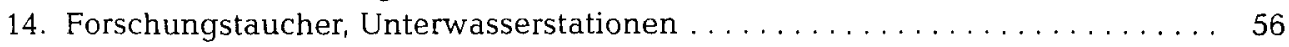

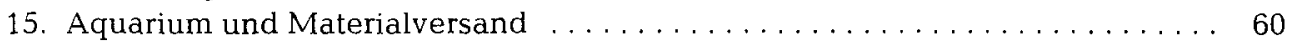

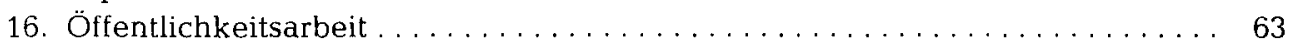

17. Gastforscher, Kurse und Studenten $\ldots \ldots \ldots \ldots \ldots \ldots \ldots \ldots \ldots \ldots \ldots$

18. Die BAH 1972: Abteilungen, interdisziplinäre Forschung, Kollegium und

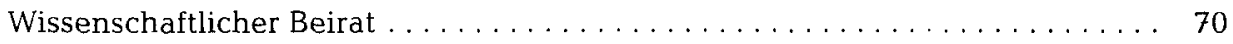

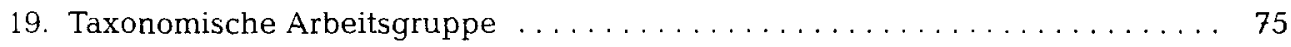

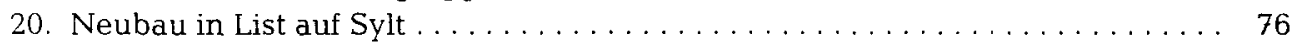

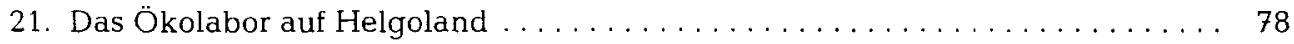

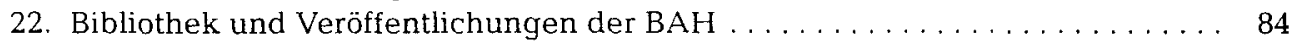

23. Neubau der Zentrale Hamburg und Verabschiedung von Prof. Kinne . . . . . 86

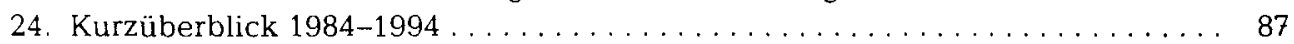

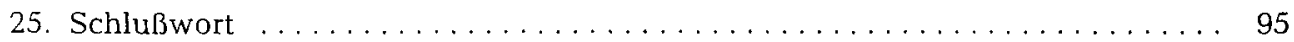

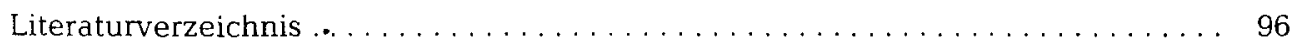

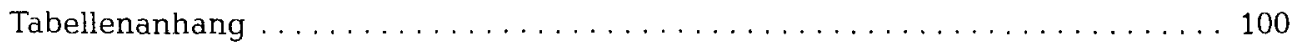

Errata in Helgoländer Meeresuntersuchungen 47 (Suppl.), 1-182 (1993) (Petra Werner: Die Gründung der Königlichen Biologischen Anstalt auf Helgoland und ihre

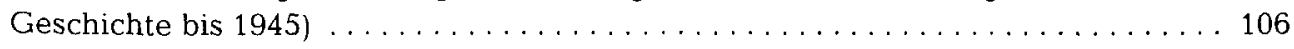




\section{Das Ende des Zweiten Weltkriegs auf Helgoland}

Auf der Insel Helgoland kam es in den letzten Kriegsmonaten für die Zivilbevölkerung regelmäßig zu nächtlichen Bunkeraufenthalten bei Überflügen starker Bomberverbände und zum Aufgebot des "Volkssturms", zu dem auch der Direktor der BAH, Prof. Arthur Hagmeier, dienstverpflichtet war. Sein Rundbrief an Betriebsangehörige und Freunde vom 15.11.1944 berichtete: „Bei dem Bombenangriff und Brand des Unterlandes am 15.10.44 brannten das Museum und das Studentenheim nieder, ohne daß etwas gerettet werden konnte. Das Hauptgebäude war in großer Gefahr, auch vom Feuer erfaßt zu werden. Es gelang jedoch dem Selbstschutz, den Brand abzuhalten. Durch Bomben sind die Glaskammer, der große Kurssaal und die Hochbehälter für das Aquarium mitsamt den verschiedenen Leitungen zerstört. Der Schaden wird jetzt so repariert, daß das Gebäude wieder benutzt werden kann. Wir arbeiten also unverdrossen weiter...".

Der Rundbrief des Direktors vom 28. Februar 1945 berichtete von Schicksalen der zum Teil weit verstreuten, eingezogenen Mitarbeiter. Dr. Ulrich Schmidt und Dr. Adolf Kotthaus waren in Küstenstellungen, Dr. Johannes Lundbeck und Dr. Horst Aurich an der Westfront. Hinrich Kanje fuhr auf einem Rheinschiff und Nickels Wichers auf der Oder. Andreas Holtmann war zur Ausheilung seiner Verwundung einige Wochen auf Helgoland, Prof. Rudolf Drost war in Northeim (Wehrmeldeamt), Dr. Peter Kornmann in Greifswald (Marineobservatorium), Dr. Bernhard Werner bei Wiesbaden. Dr. Erich Wohlenberg, Dr. Rudolf Kändler und Dr. Hubert Caspers arbeiteten im wissenschaftlichen Marinedienst. Herr Birkenhagen war noch in Norwegen, Willi Hamkens in Kurland, Heinrich Herzog in Holland, Max Dähn in Rendsburg, Herr Bub in der Slowakei. Dr. Clemens Künne war auf Walcheren in Gefangenschaft geraten, Artur Krüß wurde im Osten vermißt, Henry Bartz lag in einem französischen Lazarett, Jürgen Barnbeck war schwer verwundet. Friedrich Reimers wurde auf der Elbe durch Tiefflieger getötet. Auf Helgoland verstarben in hohem Alter der Vater und Onkel des Fischmeisters Jacob Holtmann, Andreas Holtmann und dessen Bruder Reincke Holtmann, wobei Reincke Holtmann viele Jahre mit dem Nordseemuseum und der BAH verbunden war. Auf Helgoland waren noch folgende Mitarbeiter als Soldaten: Paul Claasen, Rudolf Fichte, John Herzog, Paul-Heinz Sahling, Peter Singer. Beim Volkssturm auf Helgoland waren eingezogen: Dr. Adolf Bückmann, Prof. Hagmeier, Jacob Peter Holtmann, Peter Krüß, Reimer Rickmers und Peter Singer II. Am Ende des Rundbriefs des Direktors wurde schließlich von guten Dorschfängen im milden Winter und vom "Franzosendorsch" Gadus luscus berichtet, der im Dezember und Januar in der Tiefen Rinne gefangen wurde und als "Portionsfisch " bei der Massenverpflegung auf Helgoland sehr beliebt war.

Am 18. 4. $1945 \mathrm{kam}$ es zu Unruhen auf der Insel. SS-Einheiten waren vom Festland gekommen, um Bestrebungen einer Gruppe von Soldaten und Zivilisten zu unterdrücken, die die Insel kampflos übergeben wollte. Landesverrat nannte es die Obrigkeit und vereitelte und bestrafte grausam diese einzig vernünftige Handlung in diesem Stadium des Krieges. Am Mittag des gleichen Tages wurden Insel und Düne von Bomberverbänden angegriffen und nahezu zwei Stunden lang mit einem dichten Bombenteppich belegt. Von der Ortschaft blieben Ruinen und ein mit Trümmern übersätes Kraterfeld. Die Zivilbevölkerung, die im Felsen überlebte, hatte keine Bleibe mehr und wurde, nur mit dem Nötigsten versehen, zum Festland gebracht. 
Die militärische Besatzung sowie die dienstverpflichteten Helgoländer richteten sich in den Bunkern ein, und die Versorgung wurde wieder geregelt. Es gab noch einige Male Fliegeralarm und schwere Bomben, aber auch täglich Fortschritte bei den Aufräumungsarbeiten und die Hoffnung, das Ende des Krieges zu erleben. Dann kam die Nachricht von der Kapitulation, auf einmal brauchte man keine Furcht vor Angriffen mehr zu haben, man konnte den Frühling außerhalb des Bunkers wahrnehmen und dachte an ein Weiterleben auf der Insel und den Wiederaufbau. Diese Hoffnung auf eine glückliche Zukunft auf Helgoland wurde durch den Befehl der Besatzungsmacht zur Räumung bitter enttäuscht. Wie schon bei der Evakuierung des ersten Teils der Helgoländer durfte man nur soviel mitnehmen, wie man tragen konnte. Zurück blieb aus den Trümmern geborgene und zum großen Teil in den Bunkern untergestellte Habe, so im schwer beschädigten Gebäude der BAH (Abb. 1) viel wertvolles Inventar und Material von jahrzehntelanger Arbeit.

\section{Der Neuanfang auf dem Festland (Wedel, Altona, Cuxhaven)}

Die Mehrheit der Helgoländer wurde zunächst in dem für sie zuständigen Kreis Pinneberg behelfsmäßig untergebracht. Viele blieben in Schulau oder Wedel, wo der Transport am 13.5.1945 landete. Prof. Hagmeier und Dr. Bückmann fanden mit ihren Angehörigen am Stadtrand von Wedel in Baumschulbetrieben Unterkunft. Von dort ver-

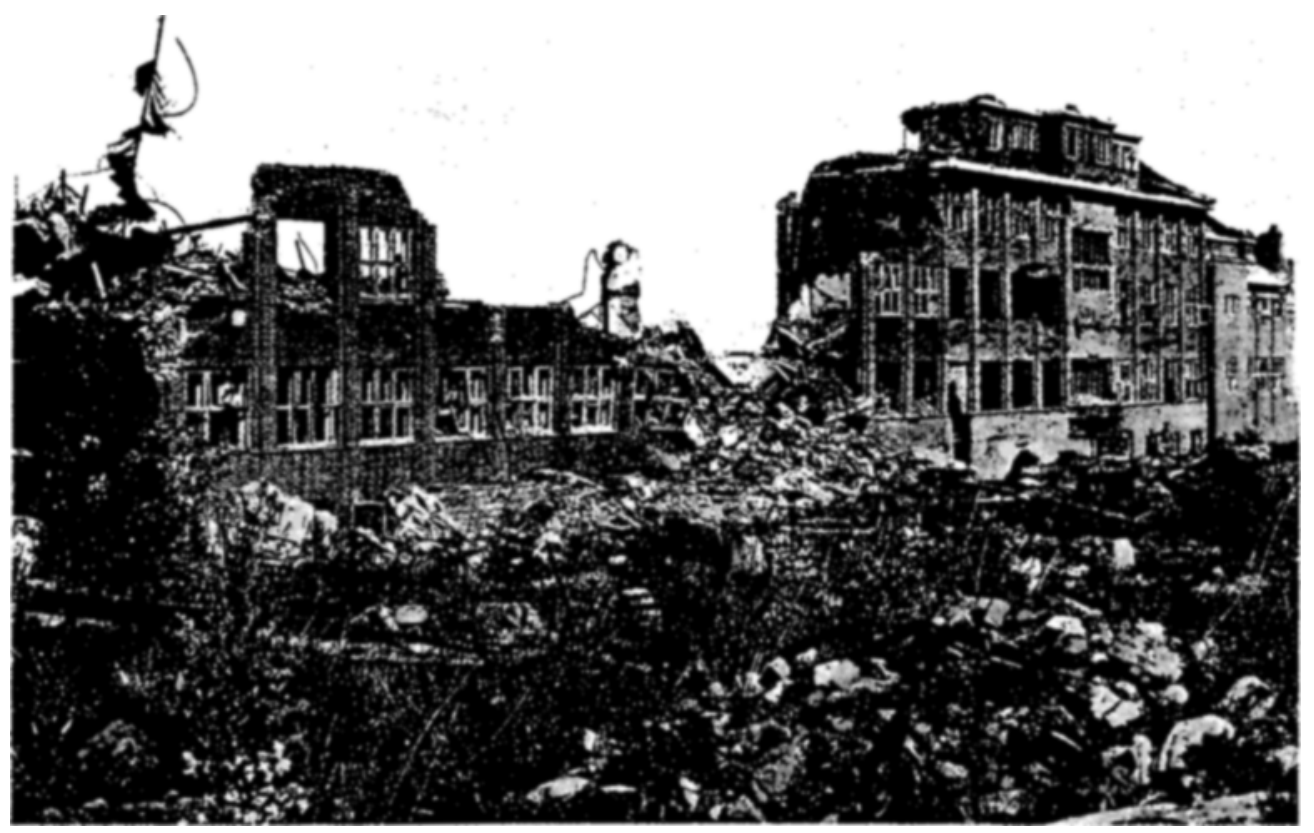

Abb. 1. Die Ruine der BAH im Juni 1946. Photo: Archiv der BAH. 
suchte Prof. Hagmeier, das zu retten und neu zu gestalten, was von der BAH übrig geblieben war.

Ein Raum in einem Schuppen der Gärtnerei Hermann Cordes wurde zur "Geschäftsstelle der Biologischen Anstalt Helgoland, Wedel (Holst.), Lülanden 4“. Von dort wurde die Verbindung mit der Verwaltung der Provinz Schleswig-Holstein hergestellt, die für die ehemalig preußische BAH zuständig war. In Wedel meldeten sich die Mitarbeiter aus den verschiedenen Orten, in denen sie mit ihren Familien Aufnahme gefunden hatten oder wenn sie aus der Kriegsgefangenschaft zurückkamen. Die Beziehungen zu den Hamburger Kollegen wurden wieder aufgenommen, die biologisch-statistischen Untersuchungen der BAH an den Fischmärkten in Hamburg und Cuxhaven organisiert. Seit Juni 1945 gab es die Fischereibiologische Arbeitsstelle der BAH am Altonaer Fischmarkt sowie die Fischereibiologische Arbeitsstelle und Versandstation der BAH in der früheren Fischlehrküche am Fischmarkt Cuxhaven, Halle VII. Die amtliche Anlandestatistik wurde wieder eingerichtet, und die Übernahme der ehemaligen Austernstation auf dem Ellenbogen der Insel Sylt wurde in die Wege geleitet. Zur Hilfe bei der umfangreichen Korrespondenz kam Paul-Heinz Sahling unter schwierigen Bedingungen an vielen Tagen von Esingen (bei Tornesch) nach Wedel.

Es galt zunächst, die Bedeutung Helgolands für die Wissenschaft bei Besatzungsmächten und deutschen Behörden deutlich zu machen und eine weitere Zerstörung der Insel zu verhindern. Eine erste Denkschrift von Prof. Hagmeier, "Die Weiterführung der Biologischen Anstalt auf Helgoland" vom 15.9. 1945, beschrieb die bisherigen Forschungen und Erfolge und betont die Dringlichkeit der Arbeiten für Meeresbiologie, Fischerei und Lehre sowie für eine rationelle Ausnutzung der Nahrungsreserven, für die es an der Biologischen Anstalt erfahrene Fachleute gab. „Kein anderes Institut könnte diese Aufgaben mit gleicher Erfolgsaussicht übernehmen“. Die Kollegen an den Universitäten, die früher als Gastforscher und mit Studenten nach Helgoland gekommen waren, wurden gebeten, das Überleben der BAH zu unterstützen. Besonders aktiv reagierten Prof. Berthold Klatt in Hamburg sowie Prof. Wolfgang von Buddenbrock in Mainz, der seit 1924 durch Sommerkurse mit der BAH verbunden war. In Hamburg wurde schon im Dezember 1945 von Prof. Hagmeier eine Erklärung über Rektor und Senat der Universität an die Schulverwaltung eingereicht. „Es liegt im dringenden Interesse der deutschen Universitäten insgesamt, daß den Wiederaufbaubestrebungen der Biologischen Anstalt Helgoland alle Unterstützung gewährt wird. Die Studierenden der Biologie sollten unbedingt das Meeresleben an Ort und Stelle studieren ...". Prof. von Buddenbrock richtete 1946 ein Rundschreiben an die deutschen Universitäten mit der dringenden Bitte um finanzielle Hilfe in der Aufbauphase der BAH. Es fiel den Verwaltungen nach den schweren Kriegsschäden im Lande sehr schwer, Geld dafür zu erübrigen. Aber es kamen Beiträge von den Universitäten Hamburg, Heidelberg, Marburg, Freiburg, Gießen, von der Anschütz-Kämpfer-Stiftung in München und, als größter Betrag von 10000 RM im Mai 1948, von der Forschungsgemeinschaft des Landes Nordrhein-Westfalen für die Anschaffung eines neuen Motorboots.

Der Leiter der ornithologischen Abteilung, Prof. Drost, war nach Göttingen verschlagen worden und suchte eigene Wege, die vorher zur BAH gehörige Vogelwarte Helgoland zu retten. Unterstützt von seinem Vorgänger Dr. Hugo Weigold, fand Prof. Drost zum 1.4.1946 einen neuen Träger mit dem "Oberpräsidium der Provinz Hannover, Hauptabteilung Kultus" und erhielt damit ein selbständiges "Institut für Vogelfor- 
schung “, das nach behelfsmäßigen Unterkünften 1947 ein ehemaliges Marinegebäude in Wilhelmshaven übernehmen konnte (Vauk, 1977).

Die Forschungsarbeiten der BAH wurden im Juni 1945 an den Fischmärkten durch die Untersuchung der Anlandungen fortgesetzt (Bückmann, 1948; Lundbeck, 1949). Im Herbst 1945 wurde mit Muscheluntersuchungen im nordfriesischen Wattenmeer zur Erfassung der Bestände und mit Messungen des Wachstums zur Förderung von Kultivierung und Nahrungsproduktion begonnen. Mies- und Klaffmuscheln (Mytilus edulis und Mya arenaria) waren als zusätzliche Nahrung sehr begehrt und wurden an vielen Stellen der deutschen Nordseeküste geerntet (Kühl, 1950). Andreas Holtmann registrierte neben seiner Tätigkeit am Fischmarkt die Muschelvorkommen im Cuxhavener Watt und beobachtete ein Mya-Feld am Wattweg Duhnen-Neuwerk, an dem Muschelgräber der Firma Lohmann \& Co., Cuxhaven, von März bis Dezember 1946 tätig waren. Im Hochsommer wurde dabei zeitweise ein Raupenfahrzeug zum Abtransport eingesetzt. Im Laufe des Jahres konnten an 160 Arbeitstagen von durchschnittlich 19 Mann etwa $213 \mathrm{t}$ Rohmuscheln ausgegraben werden, die $60 \mathrm{t}$ Fleisch enthielten. Die Hamburger Firma Eduard Ehlert \& Sohn verarbeitete diese Muscheln wie auch die seit September 1945 aus dem Büsumer Watt gelieferten Klaffmuscheln zu Konserven für die menschliche Ernährung. Zu Untersuchungen von Protein-, Glykogen- und Fettgehalt wurden Proben aus dem Sylter und Cuxhavener Watt an Dr. Johannes Henschel geschickt, der die Analysen am Hygienischen Institut in Hamburg ausführte (Henschel, 1952).

Der Abbau der Mya-Bestände, den es bei Cuxhaven auch schon nach dem 1. Weltkrieg gegeben hat, führte zu Anfragen des Fischereiamts Altona (Dr. Heidrich) und des schleswig-holsteinischen Landesfischereiverbandes (Dr. Meschkat) an die BAH, ob die Verordnung von Schonzeiten sinnvoll wäre. Prof. Hagmeier antwortete, daß nach bisherigen Kenntnissen die Klaffmuscheln sehr langsam wüchsen, die Ernte sich erst nach 7-8 Jahren lohne und ein Mindestmaß von etwa $7 \mathrm{~cm}$ für die Nutzung empfehlenswert sei. Zugleich bot Prof. Hagmeier in seinem Brief vom 16.5.1946 an den Fischereiverband die Durchführung von Untersuchungen über die Muschelbestände gegen eine Erstattung der Kosten an und nannte den heute bescheiden anmutenden Betrag von $1000 \mathrm{RM}$. Er schlug auch vor, die Verwertung der Klaffmuscheln zu prüfen sowie zu optimieren und damit eine entsprechende Untersuchung der Herzmuschel zu verbinden. Im November 1946 schickte Prof. Hagmeier an das Zentralamt für Ernährung und Landwirtschaft in der britischen Zone (Dr. Gerhard Meseck) zwei Zusammenstellungen über die Verwendung von Mies- und Klaffmuscheln im Haushalt nach Untersuchungen der Biologischen Anstalt, die gerne zur Verbreitung angenommen wurden. Die Schriften enthielten Empfehlungen für die Gewinnung und Reinigung von Muscheln, für die Zubereitung als gekochte, gebackene, geräucherte und zu Klößen, Paste und Suppen verarbeitete Muscheln mit passenden Beigaben. Dafür wurden viele Rezepte aus einer Flugschrift aus dem 1. Weltkrieg verwandt, an der Prof. Ehrenbaum mitgearbeitet hatte.

In einer späteren Stellungnahme von Prof. Hagmeier im Oktober 1948 hieß es: „Bei der Miesmuschel haben unsere Untersuchungen ergeben, daß eine ausschließliche Fischerei die Naturbänke in kurzer Zeit so verarmt, daß sich eine weitere Befischung nicht mehr lohnt. ... Die im Wattenmeer vorkommenden Miesmuscheln können nur dann auf die Dauer ein wertvolles Nahrungsmittel für die Bevölkerung werden, wenn man sie als Rohmaterial für die Kultur benutzt, also sortierte Saatmuscheln auslegt, pflegt und sie erntet, wenn sie die richtige Beschaffenheit erlangt haben. ... Dann gibt es Erträge, die 
von keiner Landwirtschaft und keiner Gartenkultur erreicht werden, nämlich 1200 bis $1500 \mathrm{dz}$ je ha".

Im August 1946 hatten endlich die Eingaben zur Bergung der auf Helgoland verbliebenen Gegenstände Erfolg. Eine erste Fahrt zur Sichtung des Vorhandenen fand mit Lt. Commander Konow als Verbindungsoffizier statt. Da das Peilboot "Ahne" des Deutschen Hydrographischen Instituts wegen schlechten Wetters nicht nach Cuxhaven kommen konnte, fuhr man zunächst am 11. 8. 1946 mit dem britischen Minensucher "290" bis zum Feuerschiff "Elbe II“, wo auf Anraten des Lotsen wegen des Seegangs umgekehrt wurde. Am 13.8. 1946 glückte die Überfahrt auf dem Minensucher "224“, und das Boot konnte an der "Wasserstelle" neben dem unzerstörten U-Boot-Bunker anlegen.

Die ganze Ortschaft war dicht bewachsen, das Unterland lag unter mannshohem Honigklee. Man kam nur langsam zwischen Trichtern und Trümmem voran. An den übriggebliebenen Häusern und ihrem Inventar waren große Schäden durch Plünderungen und Feuer entstanden. Auch im Institutsgebäude der BAH war es erschütternd zu sehen, wie Schränke und Möbel aufgebrochen und zerschlagen waren und unersetzliche biologische Proben, Manuskripte sowie wertvolle Bücher mutwillig und sinnlos vernichtet am Boden lagen. Im Aquarium waren die großen Scheiben durch Schüsse zersplittert. Von den im Bunker aufbewahrten, besonders wertvollen Aufzeichnungen, die im Mai 1945 zurückgelassen werden mußten, fanden sich nur noch wenige Journale von Fahrten der "Poseidon". Mittags mußte das Minensuchboot bei stark auffrischendem Wind schon wieder auslaufen. Es reichte nur zu einem flüchtigen Überblick!

Am 23. 8.1946 begann eine echte Bergungsfahrt mit dem Vermessungsschiff "Teutoburger Wald" des Deutschen Hydrographischen Instituts. Die BAH-Mitarbeiter Paul Claasen, John Herzog, Jacob und Andreas Holtmann, Hinrich Kanje, Peter Krüß, Peter Singer und Prof. Hagmeier wurden von der Besatzung unterstützt, und das kleine Schiff kehrte voll beladen zurück. Aber immer noch mußte vieles für die Arbeit der BAH Nützliche wie Möbel, Motoren, Installationen und Sammlungen zurückgelassen werden.

Verständnisvoller Ansprechpartner bei der Besatzungsmacht war damals Dr. James Norman Carruthers (1895-1973). In seiner Festrede zum 75. Geburtstag der BAH schildert er seinen ersten Kontakt mit Helgoland und Prof. Mielck kurz nach dem 1. Weltkrieg. Carruthers wurde durch seine Arbeiten über die Strömungen in der Nordsee bekannt. Im Zweiten Weltkrieg war er Ozeanograph in der britischen Admiralität und danach beauftragt, über Arbeiten und Fortschritte der Ozeanographie in Deutschland zu berichten und Vorschläge zur Reorganisation der deutschen Meeresforschung zu machen (Ramster, 1975). Dank seiner Bemühungen wurde das Deutsche Hydrographische Institut (DHI) 1945 zur Weiterführung der deutschen hydrographischen Dienste gegründet, mit Dr. Böhnecke (1896-1981) als erstem Präsidenten.

Der BAH wurde das Hilfspeilboot "Uthörn" des DHI zur Nutzung zugesprochen. Zur gleichen Zeit bekam das Institut für Meereskunde in Kiel das Schwesterschiff "Südfall". Im November 1946 konnte Prof. Hagmeier von List aus die "Uthörn" an ihrem Liegeplatz Tollerort im Hamburger Hafen besichtigen. Umbau und Ausrüstung des Kriegsfischkutters erforderten noch einige Mühe, verursachten Kosten von 102000 RM und zogen sich bis zum Frühjahr 1948 hin. Die Abrechnung der Dienstreise vom Ellenbogen zu dieser Besichtigung, verbunden mit Etatsbesprechungen bei der Kieler Landesregierung und Dienstgeschäften in Hamburg, ist erhalten. Die Dienstreise begann mit der Fahrt des kleinen Stationsbootes "Liuv" nach List am 11.11.1946 nachmittags, enthielt eine wei- 
tere Schiffsreise Rendsburg-Kiel und eine zeitaufwendige Rückreise am 19.11.: Wedel ab 5.24, Altona ab 6.30, Westerland an 15.15, Ellenbogen an 21.00 nach zweistündigem Fußmarsch. Die Auslagen betrugen an Fahrtkosten 49,40 RM und an Nebenausgaben, einschließlich Übernachtungen, 23,23 RM.

Das bei Kriegsbeginn noch neue Forschungsschiff der Biologischen Anstalt, die "Makrele", mußte beschädigt auf Helgoland zurückgelassen werden und wurde von der Besatzungsmacht beschlagnahmt. Im Schriftwechsel zwischen Andreas Holtmann und Arthur Hagmeier 1947 wird die "Makrele" noch erwähnt. Sie lag einige Jahre ohne Verwendung in Cuxhaven. Für die BAH durfte sie nicht wieder in Betrieb genommen werden. Eine Notiz im Hamburger Abendblatt vom 13.5. 1950 besagte, daß das Schiff zu der Zeit auf den Howaldtswerken in Kiel für die norwegische Regierung zu einem Ausbildungsschiff für Seefahrtsschulen umgebaut wurde und den Namen „Sjøveien " tragen sollte.

Ende Mai 1949 wurde die Dienststelle Cuxhaven der BAH mit Fischereiforschung und Versandstelle aufgelöst. Die Fischereiforschung bekam ein Labor in Bremerhaven, und der Versand von biologischem Material wurde von List aus durchgeführt. Der Oberpräparator John Hinrichs, schon über 70 Jahre alt, behielt einen Arbeitsplatz bei Dr. Heinrich Kühl in der Außenstelle des Instituts für Küsten- und Binnenfischerei in Cuxhaven. Dort beobachtete er bis zu seinem Tode im Oktober 1951 die täglichen Planktonproben und half mit seinem großen Wissen. Für die Institutsmitglieder war er ein lieber, väterlicher Freund. Leider brachte er die Erinnerungen aus seinem langen und reichen Leben mit der BAH nicht mehr zu Papier.

\section{Der Wiederbeginn auf Sylt}

Im Jahr 1946 konnte man wieder von einem arbeitenden Institut sprechen. Die Austernfischereianlage ("Austerngehöft") auf dem Ostteil des Ellenbogens der Insel Sylt (Abb. 2) war im Jahr 1936 von der Deutschen Luftwaffe unter Mitwirkung des Hochbauamtes Flensburg als Ersatz für die größere fiskalische Austernanlage im Dorf List erbaut worden, deren Gelände und Gebäude für die Einrichtung des Fliegerhorstes List beansprucht wurden. Das neue Austerngehöft wurde der Domänenverwaltung in Schleswig zur Verfügung gestellt und diente dem Pächter der im nördlichen Sylter Wattenmeer gelegenen fiskalischen Austernbänke als Wohn- und Betriebsstätte. Außerdem war das Austerngehöft der Sitz des Zweiglaboratoriums List der BAH auf Helgoland und von 1941 an gleichzeitig der Sitz der Muschelforschungsstelle Sylt der Reichsanstalt für Fischerei. Die BAH führte hier 1937 bis zum Kriegsanfang ihre Versuche zur künstlichen Austernzucht durch, die bis zum Beginn der praktischen Ausnutzung gediehen waren.

Nach besuchsweisen Aufenthalten der Biologen im Jahr 1945 wurde das Laboratorium auf dem Ellenbogen im März 1946 wieder in Betrieb genommen und weiter ausgebaut, nachdem die Landesverwaltung Schleswig-Holstein der BAH das ganze Austerngehöft überlassen hatte und der frühere Pächter Dethlefs seinen Teil räumen mußte. Seit Ende Juli 1946 standen die Gebäude und Anlagen des Austerngehöftes wie folgt zur Verfügung. Das frühere Wohngebäude des Austernpächters, der drei Gasträume als "Austernprobierstuben“ im Erdgeschoß eingerichtet hatte, enthielt neben der gemeinsamen Küche zwei als Laboratorien eingerichtete, nach Norden und Osten gelegene 


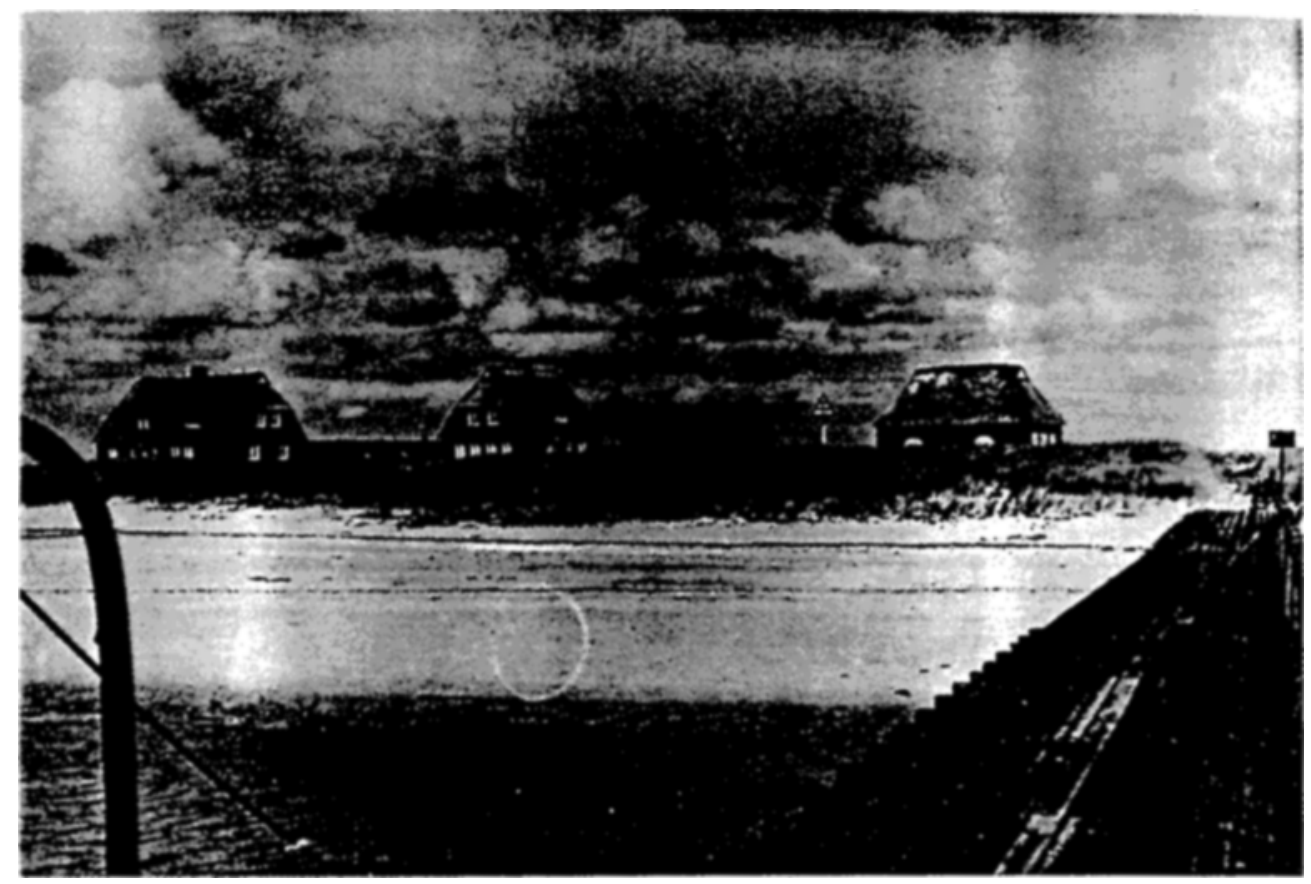

Abb. 2. Das "Austerngehöft" auf dem Ellenbogen (Sylt). Photo: Andreas Holtmann.

Räume, das Verwaltungszimmer und das große, nach Süden gelegene gemeinsame Eßzimmer, in dem auch Teile der Bibliothek untergebracht waren. Die übrigen sechs Zimmer dienten als Wohn- und Schlafzimmer für die Mitarbeiter und in beschränktem Umfang für Gäste, die an der Station arbeiteten.

Das Wirtschaftsgebäude enthielt im Erdgeschoß das mit See- und Frischwasser versorgte Laboratorium mit einem Sterilisierraum für Seewasser, ferner den früheren "Packraum", der als Sortierraum und zeitweise behelfsmäßig als Kursraum eingerichtet war. Das Obergeschoß enthielt noch vier Zimmer, die als Wohn- und Schlafzimmer für die Mitarbeiter dienten und von denen zwei bei Bedarf als Laboratorien eingerichtet werden konnten. Im Keller befanden sich die Heizung, ein Aquarienraum mit auch im Sommer niedriger Temperatur und der frühere Aufbewahrungsraum für Konsum-Austern, dessen geräumige Becken zur Hälterung von Versand- und Kursmaterial dienten. Direkt verbunden mit dem Erdgeschoß des Wirtschaftsgebäudes war das gewächshausartige "Austernhaus" mit einer Seewasser-Filteranlage und großen Becken für die Austernzucht (Abb. 3). Behälter, Zucht- und Futterbecken waren mit säurefesten Kacheln ausgekleidet. Im Keller standen die Kolbenpumpe für die Seewasserzuleitung, eine Kreiselpumpe für das Füllen des Hochbehälters, ein Kompressor für die Belüftung der Aquarien, die Süßwasserpumpe mit Druckkessel und schließlich das Aggregat mit Akkumulatoren zur Versorgung der ganzen Anlage mit Strom.

Vom Wirtschaftsgebäude führte ein Feldbahngleis an einem geräumigen Fischereischuppen vorbei bis zur Spitze der Landungsbrücke. Unter deren Balken verlief die aus 


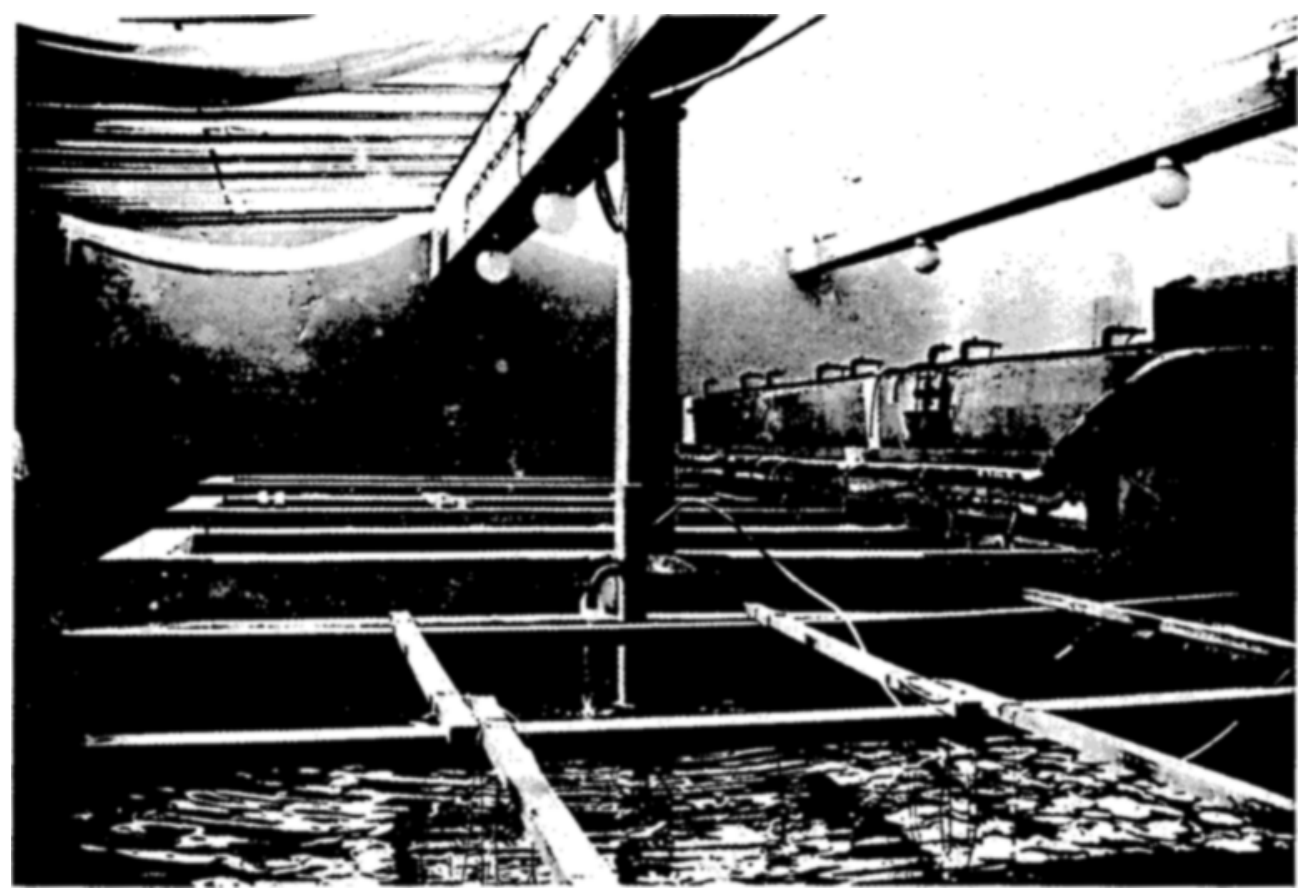

Abb. 3. Austernzuchtbecken im Austernhaus. Photo: Andreas Holtmann, etwa 1938.

Eternitrohren hergestellte Seewasser-Saugleitung. Für den Stationsbetrieb wurde das Seewasser kurz vor oder bei Tidenhochwasser zunächst über Filter in einen Tiefbehälter vor dem Austernhaus gepumpt. Zum Hochbehälter lief das Wasser in Steinzeugrohren, zu den Becken und Zapfstellen in Leitungen aus Mipolam (PVC-Rohr), eine große Verbesserung gegenüber den vorher verwandten Zelluloidrohren. „Die Lage zur See ist hier äußerst günstig, “ so schrieb Prof. Hagmeier (1951) im Zoologischen Anzeiger. "Die Station liegt geschützt in einer Nordseebucht ohne nennenswerte Süßwasserzuflüsse, gegen das Wattenmeer durch die zum Festland führenden Dämme von Röm und Sylt abgeschlossen und mit der freien Nordsee durch das breite und tiefe Lister Tief verbunden. Wassereigenschaften und Planktongehalt erinnem daher eher an die offene Nordsee als an das Wattenmeer und heben sich deutlich von denen der übrigen Nordseeküste ab. Die Seewasserversorgung der Station gestattet Züchtungen von empfindlichen Meeresorganismen, wie sie selbst auf Helgoland früher nicht möglich waren. “

Für die Unterkunft der Mitarbeiter und einen Institutsbetrieb war natürlich noch vieles zu beschaffen. Möbel, Betten und Decken kamen im April 1946 von der Marine-Intendanturstelle Sylt, sogenannte "wehrmachtseigene Unterkunftgeräte" mit einem Zeitwert von RM 1601 und einer monatlichen Miete von RM 13,35. In List waren in einem Unterkunftsgebäude des ehemaligen Seefliegerhorstes, dem "Strohdachhaus ", bescheidene Wohnräume für einige Mitarbeiter und deren Familien (Krüß, Rickmers, Sahling) angemietet worden (Abb. 4). In ein Zimmer zog die Verwaltung ein. Olaf Goemann, der Enkelsohn des Verwaltungsleiters Peter Krüß, erinnert sich an einen Winterabend. Die 


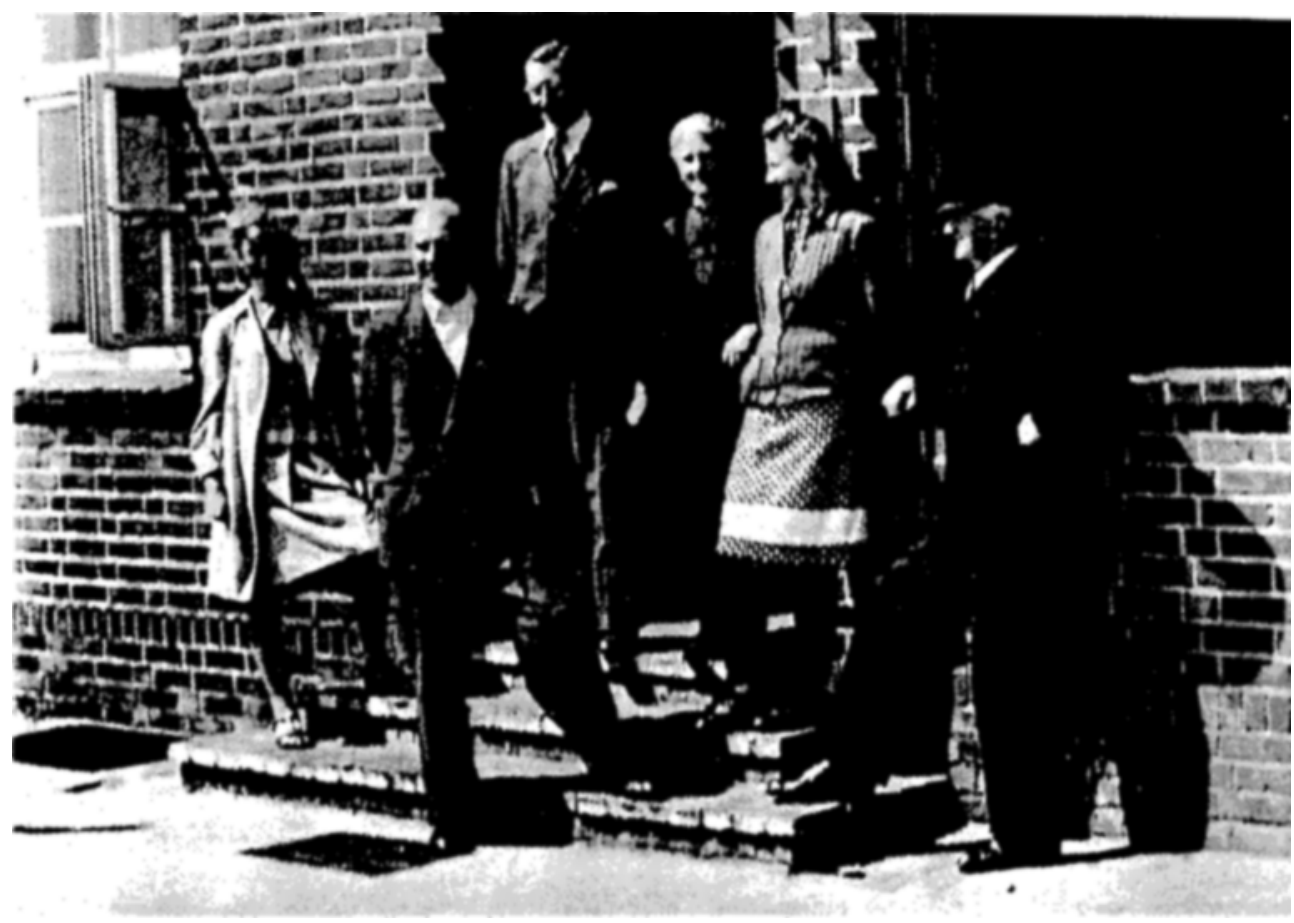

Abb. 4. Mitarbeiter der BAH und Angehörige vor dem Eingang des "Strohdachhauses", List, Hafenstraße. Von rechts nach links: Peter Krüß, Helene Goemann (Tochter von Peter Krüß), Johanna Krüß (Ehefrau von Peter Krüß), Paul-Heinz Sahling, Henry Bartz, Waltraud Herzog (Tochter von Heinrich Herzog). Photo: etwa 1950, im Besitz der Familie Goemann.

Kinder wurden in die Betten geschickt, die mit einem heißen Stein angewärmt waren, und die Erwachsenen beschäftigten sich im einzigen geheizten (Wohn-)Zimmer der Familie Krüß mit dem Schneiden und Kleben von Umschlägen aus gebrauchtem Papier für den Verwaltungsbetrieb.

Prof. Hagmeier, Dr. Werner und Heinrich Herzog wohnten in der Station auf dem Ellenbogen. Dort begeisterte die Natur die Biologen, und Dr. Wemer machte dort seinen täglichen Strandrundgang. Prof. Hagmeier schrieb am 29.5.1946 an seinen Sohn: „... Große Flächen der Wiesen in den Dünentälern sind rosa überzogen von blühenden Grasnelken, zwischen dem Helm der Sanddünen blühen Blumen mit herrlichen Farben, wie ich es früher nicht für möglich gehalten hätte ... Der Himmel zeigt am Abend und Morgen wundervolle Farben, bei klarem Wetter geht die Abendröte jetzt in die Morgenröte über, es wird kaum richtig dunkel in der Nacht. Vorhin war ich einige Zeit auf der Austernbrücke. Das Wasser stieg und war ganz klar, ein Neerstrom ging unter der Brücke weg und führte schöne Haarquallen mit sich, blaue und eine tellergroße rote, wie man sie selten sieht. Am Grunde waren kleine Schollen und Flundern zahlreich zu sehen, gegen den Strom standen große Scharen von Spierlingen, welche über den Schatten der Brücke nicht hinausschwammen ..." Am 8. 2. 1947 heißt es in einem Brief an Prof. Ernst Küster (Gießen): „Zur Zeit ist hier eine großartige Polarlandschaft. Das ganze Kö- 
nigshafengebiet ist mit Eisschollen und kleinen Eisbergen bedeckt. Im Hause ist es, da die Zentralheizungen stillgelegt sind und nicht genügend Heizmaterial für den Ofen zur Verfügung steht, allerdings sehr kalt, zwischen 4 und 10 Grad im geheizten Zimmer - es werden aber manche noch mehr frieren müssen. Wir sind nur in Sorge, daß mit dem später abgehenden Eis auch unsere Versuchsbeete und die Muschelbänke stark geschädigt werden." Nahe beim Institut und bei Niedrigwasser leicht zu erreichen lag eine reichhaltige Miesmuschelbank, die wesentlich zum Speisezettel der Biologen beitragen konnte. Mittags wurde auch für die Mitarbeiter gekocht, die jeden Tag von List zur Station kamen. Abrechnungen der "Ökonomiekasse" zeugen von sehr bescheidenen Aufwendungen.

Als erster Kurs kamen im September 1946 acht Studenten mit Dr. Karl Grell (1912-1994) aus Bonn auf den Ellenbogen, erster Gastforscher war in diesem Jahr Prof. von Buddenbrock aus Mainz. Im Sommer 1947 gab es schon 80 Arbeitsplatzbeleger, und als erster Besucher im Frühjahr kam im März 1948 Prof. Beutler aus München. Nach dem Bonner Kurs im Mai 1948 mußten jedoch wegen der Währungsreform zahlreiche Gastforscher und Kurse ihre Anmeldungen zurückziehen, und auch der für Juli angekündigte erste Anstaltskurs konnte nicht stattfinden. Trotzdem gab es 1948 über 90 Kursteilnehmer und Arbeitsplatzbeleger auf dem Ellenbogen, und der zurückgekehrte Andreas Holtmann war eine willkommene Verstärkung für die Betreuung (Abb. 5). Im Jahr 1949 kamen sogar 170 Studenten.

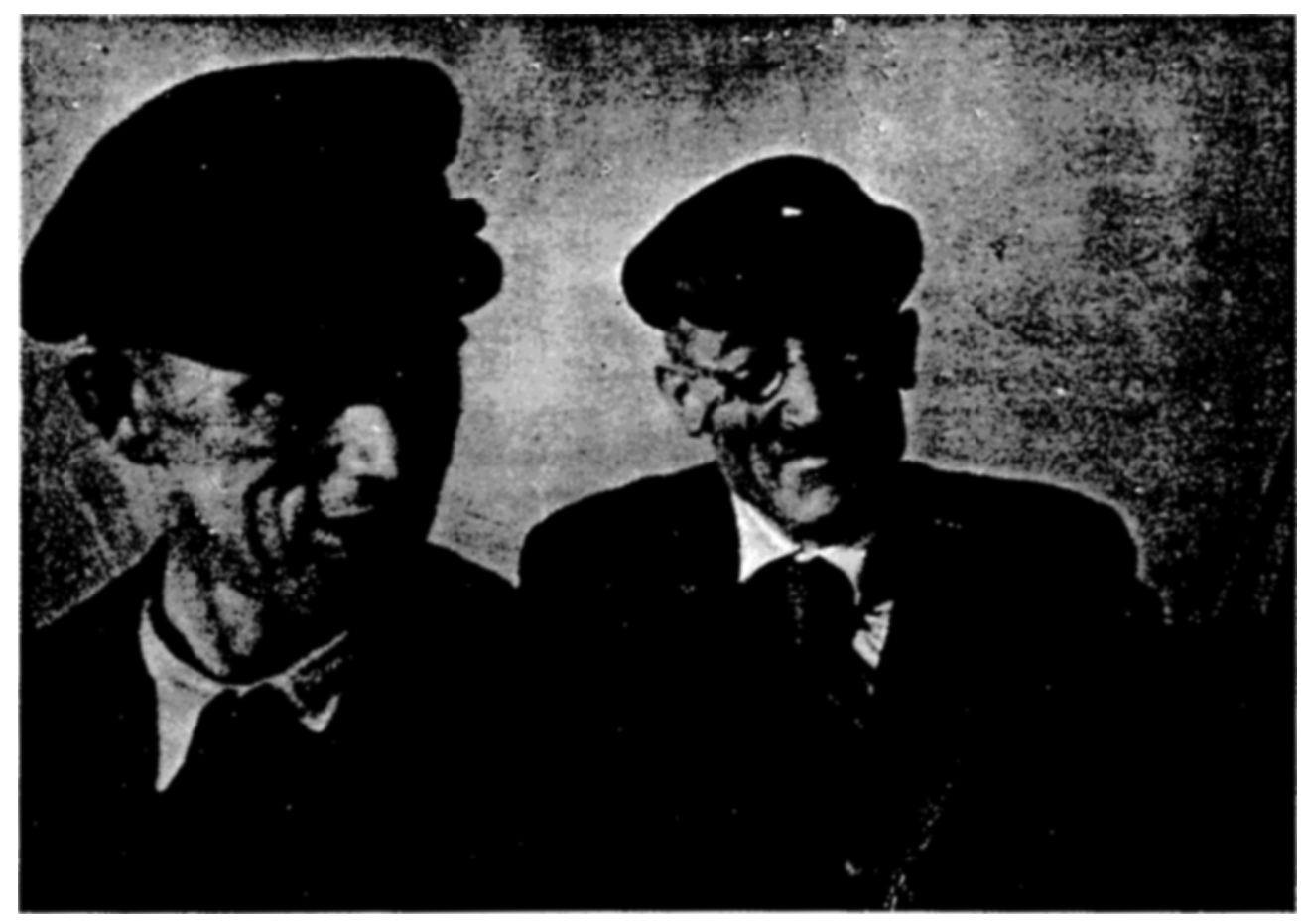

Abb. 5. Andreas Holtmann (links) und Prof. Arthur Hagmeier, Sommer 1948, Photo: E. Dorn. 


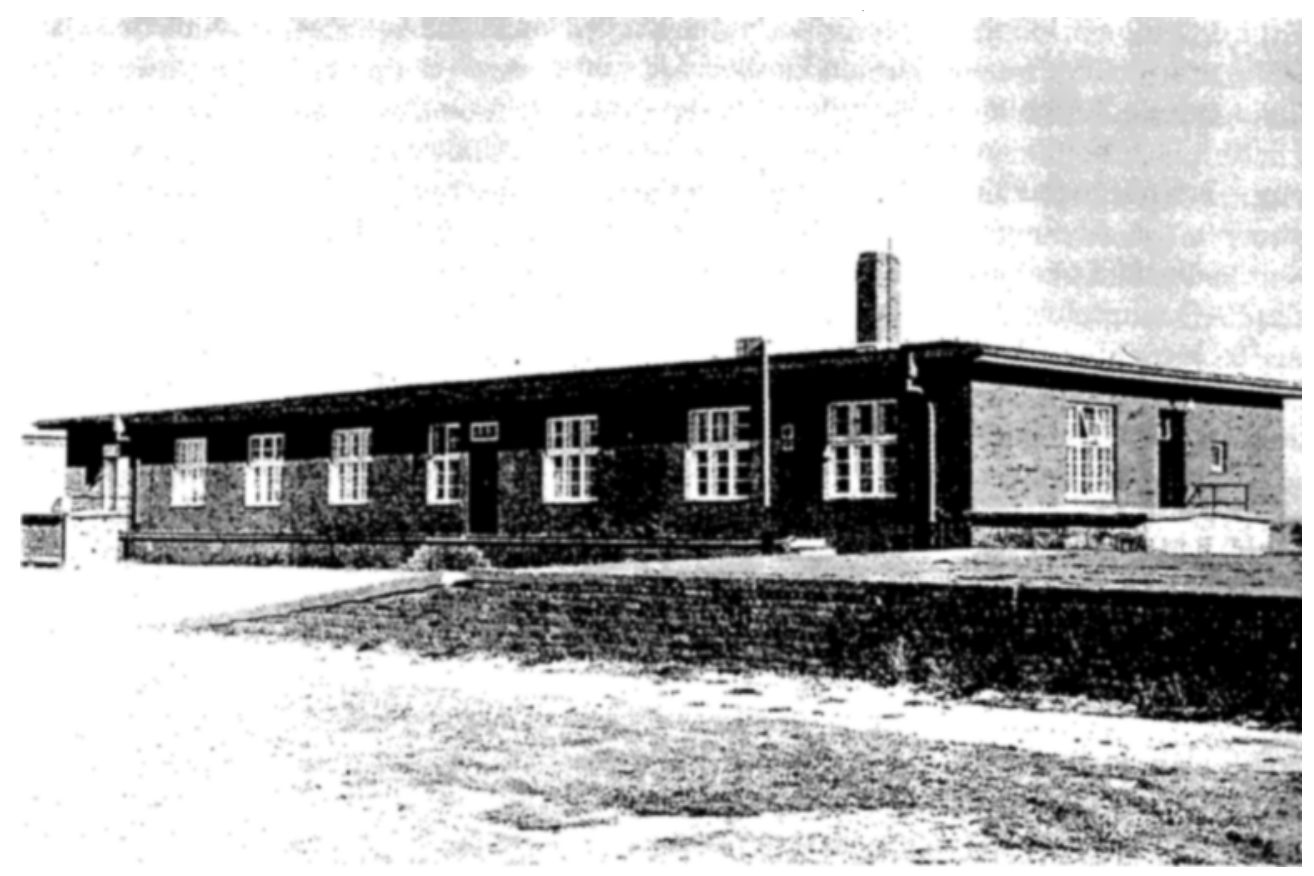

Abb. 6. Altes Laborgebäude ("Bombenbäckerei") in List. Photo: Dr. Erich Ziegelmeier.

Das Programm des BAH-Kurses für Lehrer vom 13,-27.7.1950 auf dem Ellenbogen ist erhalten (Tabelle 5). Die Dozenten waren Dr. Aurich, Prof. Hagmeier, Dr. Kornmann, Dr. Lundbeck, Dr. Werner und Dr. Erich Ziegelmeier. Angeboten wurden Ausfahrten, Exkursionen in das Watt, an den Strand und über die Insel bis zum Morsumkliff sowie zum Rantumbecken, Untersuchungen von Plankton, Krebsen, Stachelhäutern, Mollusken, Coelenteraten und Fischen, Algenstudien, Einführungen in Fischereiwissenschaft und Hydrographie, künstiiche Befruchtung bei Echinodermen, Polychaeten (Sabellaria) und Muscheln (Barnea candida) mit der Absicht, eine Vorstellung von Formen, Funktionen, Lebensweisen von Pflanzen und Tieren sowie ökologischen Zusammenhängen zu vermitteln.

Ab 1951 konnte die Station auf dem Ellenbogen etwas entlastet werden. In einem 1936/37 errichteten früheren Wehrmachtsgebäude am Lister Hafen, in dem man Betonkörper für Übungszielwürfe im Wattenmeer hergestellt hatte (deshalb "Bombenbäckerei "genannt, Abb. 6), wurden Laboratorien und zwei Kursräume für je 15 Teilnehmer eingerichtet. Dank der Mitarbeit von Prof. Carl Schlieper, Kiel, konnte bei den Anstaltskursen auch die Physiologie der Meerestiere berücksichtigt werden. Prof. Wilhelm Josef Schmidt, Gießen, hielt 1952 einen Kurs zur Einführung in die Polarisationsmikroskopie.

Auch die Ausbildung von Laboranten wurde wieder aufgenommen. Zu den ersten Lehrlingen gehörten Martin Söhl (bis 1990 an der BAH), Hanna Schmidt und Peter Thomsen. Später begannen die Helgoländer Jacob K. Holtmann, Olaf Goemann, Eva-Maria 
Krüß und Inge Schmolke. Diese Mitarbeiter, die im Hause und auf See gründlich und vielseitig ausgebildet waren, wurden für die BAH sehr wertvoll und übernahmen später zum Teil leitende Funktionen. Nach dem Bundes-Angestelltentarifvertrag (BAT) wurde aber ein für die Arbeiten an der BAH genau passend ausgebildeter Laborant niedriger eingestuft als ein schulmäßig und kürzer ausgebildeter Technischer Assistent, der dann für bestimmte Aufgaben angelernt werden mußte und nicht so universell einzusetzen war. Es wurde deshalb seit den 70er Jahren den Helgoländer Interessenten an einer Tätigkeit bei der BAH empfohlen, in eine entsprechende Schule auf dem Festland zu gehen und sich als Technischer Assistent ausbilden zu lassen. Es verblieben bei der BAH nur die Ausbildungsplätze für "biologische Fischer", später "Fischwirte” genannt, mit deren Hilfe die BAH tüchtige Nachwuchskräfte erhielt. Ein wesentlicher Teil der Ausbildung erfolgte in den 80er Jahren durch unseren ehemaligen Netzmacher Nickels Wichers.

Im Jahr 1950 konnte Dr. Kornmann auf die wieder eingerichtete Botanikerstelle der BAH zurückkehren. Das Feld an der Küste der Insel Sylt war nicht so reich an Algen wie die Umgebung Helgolands (Kornmann, 1952), bot aber genügend Arten für wertvolle Arbeiten über ihre Morphologie und Entwicklung, und auch Phaeocystis wurde kultiviert und beobachtet. Von Dr. Paul Kuckuck (1866-1918) nachgelassene Aufzeichnungen über die Ectocarpaceen, deren Bearbeitung Dr. Kornmann schon vor dem Kriege vorbereitet hatte, waren wie durch ein Wunder in Kopien erhalten und konnten in List ergänzt und mit der Hilfe von Paul-Heinz Sahling herausgegeben werden. Die von Dr. Kuckuck für einige Notizen gebrauchte veraltete Stenographie mußte erst mühselig entschlüsselt werden.

In diese Zeit fallen auch Entwurf und Gestaltung zweier Schautafeln: (1) "Nahrung und Nahrungserwerb der Seefische" von Prof. Hagmeier und Dr. Ziegelmeier, mit einer Darstellung des Meeres von der Oberfläche bis zum Boden mit Algen, Seeigeln, Seesternen, Krebsen, Quallen, Futter- und Nutzfischen (Titelbild bei Werner, 1993) und (2) "Die Fanggründe der deutschen Hochseefischerei in den nordeuropäischen Meeren, ihre Ergiebigkeit und wichtigsten Nutzfische" von Dr. Lundbeck. Die Ausführung der Darstellung beider Schautafeln erfolgte durch den Worpsweder Maler Willi Ohler (1888-1975), der vor dem letzten Krieg gerne auf Helgoland gemalt und viel für die Ausschmückung von Institut und Studentenheim getan hatte.

\section{Zur Finanzierung und Trägerschaft der BAH}

Das Fortbestehen der BAH und anderer staatlicher Forschungseinrichtungen war nach Kriegsende sehr ungewiß. In einem Brief von Prof. Hagmeier, Wedel, an Dr. E. Wohlenberg, Husum, vom 2. 3. 1946 heißt es: "An der Anfrage von Herrn Dr. Fehling, “ (Kieler Kultusministerium) "ob die Biologische Anstalt Ihr Institut" (gemeint ist die Forschungsstelle des Marschenbauamts in Husum) „übernehmen könnte, bin ich vollständig unbeteiligt und habe einen derartigen Gedanken auch nicht geäußert. Es liegt aber auf der Hand, daß die Provinz Schleswig-Holstein auf Sparmaßnahmen aus ist, da sie jetzt sehr viele Institute zu betreuen hat. Nun besteht auch die Gefahr, daß ich selbst entlassen werde; für diesen Fall hatte ich mir schon überlegt, daß Sie die Biologische Anstalt übernehmen könnten." Prof. Hagmeier, der somit neben der Suche nach einem Träger für die BAH auch nach einem Nachfolger für sich selbst Ausschau hielt, schrieb am 12.9.1946 an Prof. von Buddenbrock: „Die Lage ist noch immer nicht geklärt. Wir sollten zunächst 
an das Deutsche Hydrographische Institut angeschlossen werden, das aus der früheren Deutschen Seewarte hervorgegangen ist und unter der Kontrolle der britischen Kriegsmarine arbeitet. Mit Dr. Böhnecke zusammen habe ich die Aufgaben und den Etat ausgearbeitet, aber die Direktorenkonferenz" (der vier Großmächte) "kam auf die Ansicht, $\mathrm{da} B$ die Anstalt vorwiegend Fischereiwissenschaft betrieben habe und daher dem Fishery Control Board und dem Zentralamt für Emährung und Landwirtschaft unterstellt werden müsse. Der Fischereireferent beim Zentralamt verhielt sich zunächst ablehnend und sagte, er müsse zunächst für die früher dem Reichslandwirtschaftsministerium unterstellten Institute sorgen und könne daher die Anstalt, trotz hervorragender Verdienste auf dem Fischereigebiet, auch nach dem Zusammenbruch nicht ausreichend dotieren. Auch jetzt, bei den Etatsverhandlungen, soll ich mich auf ein Minimum beschränken. Aber ohne ausreichenden Etat kann der Betrieb in keiner Hinsicht erfolgreich sein. ... Die Provinz Schleswig-Holstein hat alle Anerkennung verdient, daß sie uns bis jetzt über Wasser gehalten hat. Ich kann es ihr aber nicht verdenken, wenn sie uns jetzt wieder los sein will, sobald sich eine Zentralstelle unser annehmen kann. ... Der Friedensetat der Anstalt betrug gegen 250000 RM, der Notetat der Provinz Schleswig-Holstein gegen 100000 RM, die aber nicht ganz verwendet wurden, da noch Beamte in Kriegsgefangenschaft sind, deren Stellen im Etat bleiben müssen. Der normale Etat für eine im Personal auf weniger als die Hälfte reduzierten Anstalt ist von mir jetzt auf $160000 \mathrm{RM}$ berechnet. Ich habe aber fast keine Hoffnung, daß das Zentralamt für Ernährung und Landwirtschaft diese Summe bewilligt. Ich werde wohl weiter mit dem Notetat arbeiten müssen bzw. froh sein, wenn ich das überhaupt kann."

Ab 1. 4. 1947 sollte die Anstalt vom Zentralamt für Ernährung und Landwirtschaft betreut werden, was nicht gleich reibungslos funktionierte. In einem Brief von Andreas Holtmann, Cuxhaven, am 30. 5. 1947 an Prof. Hagmeier, Ellenbogen, heißt es. „Es ist noch kein Gehalt für April und Mai eingegangen!“ Auf Anordnung der Finanzabteilung der Militärregierung mußten Anfang Oktober 1947 die Forschungsinstitute des Ernährungsund Landwirtschaftsrates für das amerikanische und britische Besatzungsgebiet wieder von den einzelnen Ländern übernommen werden. Schleswig-Holstein, 1946 als Land aus der preußischen Provinz gebildet, wurde wieder zuständig, ohne daß in dem laufenden Jahr noch Gelder für die BAH vorgesehen waren. In diesen finanziellen Engpaß fielen gerade die hohen Rechnungen für den Forschungskutter "Uthörn" und das neue Motorboot "Ellenbogen".

Am 25. 10. 1948 konnte Prof. Hagmeier seinen Mitarbeitern bekanntgeben, daß die BAH rückwirkend ab 1.4.1948 der Verwaltung für Ernährung und Landwirtschaft des Vereinigten Wirtschaftsgebietes unterstellt war, als selbständiges Institut der Zentralanstalt für Fischerei in Hamburg. Deren Verwaltung befand sich damals in Hamburg, Klockmannhaus, und Verwaltungsleiter war Henry Bartz (Abb. 4), ein Helgoländer und früherer Mitarbeiter der BAH. Nach Umwandlung des Zentralamts für Ernährung und Landwirtschaft in das Bundesministerium für Ernährung, Landwirtschaft und Forsten im Jahr 1949 wurde die Zentralanstalt zur Bundesanstalt und 1952 zur Bundesforschungsanstalt für Fischerei. Zur Aufnahme der BAH als ein im wesentlichen mit der Grundlagenforschung befaßtes Institut in diese Bundesforschungsanstalt paßt das Wort Friedrich Heinckes: "Es gibt nur eine Wissenschaft, deren beide Teile, der theoretische und der angewandte, sich gegenseitig befruchten müssen". In vielen Briefen und Stellungnahmen kann man aber lesen, daß es schwierig war, die Selbständigkeit und den Personalbestand 
gegenüber den anderen Instituten der Bundesforschungsanstalt für Fischerei zu behaupten. So waren im März 1953 drei wissenschaftliche Beamtenstellen der BAH durch Kollegen anderer Institute der Bundesforschungsanstalt für Fischerei besetzt.

Es war auch nicht selbstverständlich, daß ein "Bundesinstitut" die traditionellen Stationsaufgaben der BAH weiterführte, wie die Betreuung von Gastforschern und Kursen und den Materialversand für die den Ländern unterstehenden Universitäten. Prof. Hagmeier bemühte sich um Verständnis bei den Bundesbehörden und um Zuschüsse bei den Universitäten. In den Akten des Hamburger Staatsarchivs gibt es darüber einige Belege. Von der Universität Hamburg wurden 1947 und 1948 je 3000 RM und 19492000 DM für den Unterrichtsbetrieb an der BAH bewilligt. Als 1950 die Nutzung zusätzlicher Gebäude in List finanziert werden sollte und die Hamburger Station auf Spiekeroog aufgegeben wurde, meldete der Dekan der mathematisch-naturwissenschaftlichen Fakultät, Prof. Remy, über den Rektor der Hamburger Schulbehörde sein Interesse an einem weiteren Ausbau der für Studenten bestimmten Einrichtungen der BAH in List an. Die Antwort der Schulbehörde (Dr. von Heppe) war der Hinweis auf den geplanten Aufbau des Max-Planck-Instituts für Meeresbiologie. Dazu war schon 1948 in Kiel überlegt worden, ob dieses Institut vielleicht auf Sylt im Rahmen einer Erweiterung der BAH verwirklicht werden könnte. Nach der Übernahme der Finanzierung der BAH im Rahmen der Zentralanstalt für Fischerei durch die Frankfurter Wirtschaftsverwaltung und die Entscheidung der Max-Planck-Gesellschaft für Wilhelmshaven verlief die Entwicklung jedoch anders. Jetzt wollte man Überschneidungen beim Ausbau der beiden Institute vermeiden und wegen der hohen Zuschüsse von der Ländergemeinschaft zur Finanzierung überregionaler Forschungseinrichtungen die BAH nicht noch zusätzlich fördern. Der Hinweis von Prof. Klatt, daß eine Ausbildung von Studenten im Max-Planck-Institut nicht möglich wäre, nützte nichts. Eine Anfrage der Schulverwaltung bei den Kultusministern der Länder, deren Universitäten in List Kurse abhielten, ergab, daß es von dort keine Unterstützungen für die BAH gäbe. Dr. von Heppe entschied also, daß von der Hamburger Universität nur Zuschüsse für Kurse und den Ankauf von Unterrichtsmaterial aus Fakultätsmitteln gezahlt werden dürften.

Die seit dem 1.11. 1934 bestehende Arbeitsgemeinschaft zwischen der BAH und der Mathematisch-Naturwissenschaftlichen Fakultät der Hamburger Universität wurde 1948 "wiederbelebt". Es wurden Vorträge von Mitarbeitern der BAH im Wintersemester in Hamburg vereinbart, wobei "clearance certificates" (Entlastungszeugnisse) vorgelegt werden mußten. Beispiele für derartige Vorträge sind: 17.1.1949 Kustos Dr. Künne: „Verbreitung der Planktontiere durch Meeresströmungen"; 31.1.1949: Wiss. Asssistent Dr. Werner: "Einschleppung und Biologie der amerikanischen Pantoffelschnecke in das deutsche Wattenmeer“; 7. 2. 1949 Wiss. Assistent Dr. Aurich: "Die Fischbrut als Bestandteil des Meeresplanktons". Zugleich wurden Kurse im Sommersemester in List verabredet. Ein Brief dazu von Prof. Hagmeier vom 28.5.1948 beleuchtet die noch immer schwierigen Lebensbedingungen. "Für das nächste Sommersemester (1949) kann ein meeresbiologisches Praktikum," (der BAH auf dem Ellenbogen) "ganztägig, mit gelegentlichen Exkursionen angekündigt werden. Die Zahl der Teilnehmer muß vorläufig auf drei beschränkt werden, hauptsächlich wegen der Unterbringung und der Verpflegung. Bis zu drei Studierende können in einem Zimmer der Hauptstation wohnen und dort auch essen, wenn sie Lebensmittelmarken und die Kartoffeln und außerdem die Gemüsekarte mitbringen. Die Kosten für Wohnen und Zubereitung der Speisen werden 
sich in geringen Beträgen halten. Solange kein zweites Zimmer zur Verfügung steht, können keine Studentinnen aufgenommen werden."

Zur ideellen und finanziellen Unterstützung des Wiederauf- und Ausbaus der Einrichtungen für Studenten und Gastforscher wurde Mitte 1951 zur Gründung der "Gesellschaft der Freunde der Biologischen Anstalt Helgoland" aufgerufen. Unterzeichner waren die Professoren Wolfgang von Buddenbrock (Mainz), Wulf Emmo Ankel (Darmstadt), Berthold Klatt (Hamburg), Wilhelm Josef Schmidt und Ernst Küster (Giessen), Wilhelm Kuhl (Frankfurt/Main), W. Zimmermann (Tübingen), H. Kappert und E. Tiege (Berlin-Dahlem) sowie Kurt Hoffmann (Kiel). Der Aufruf wurde zusammen mit der "Notiz über die BAH nach dem Kriege" von Prof. Hagmeier verschickt.

Auch Prof. Hase (Berlin) schrieb im Namen der Deutschen Zoologischen Gesellschaft und der Deutschen Botanischen Gesellschaft einen Aufruf zur Unterstützung der BAH. Sein Amtsnachfolger als Vorsitzender der Deutschen Zoologischen Gesellschaft, Prof. Ankel (Darmstadt), wandte sich deswegen im Dezember 1951 an die Kultusminister-Konferenz in Wiesbaden und ein Jahr später sogar an den Bundespräsidenten, Prof. Heuss. Prof. Ankel wertete die zukünftige BAH auf Helgoland als ein "Neapel des Nordens". Er war besonders um den Fortbestand der Grundlagenwissenschaft unter dem Ernährungsministerium besorgt, und er sah das Gleichgewicht zwischen Grundlagen- und Zweckforschung bedroht durch die Berufung eines Fischereibiologen als Direktor der Biologischen Anstalt, während die Abteilung für Grundlagenwissenschaft nur von einem Oberregierungsrat geleitet werden sollte (Geus \& Querner, 1990). Prof. Heuss antwortete umgehend, daß er in Ressortauseinandersetzungen über die Gestaltung einer naturwissenschaftlichen Forschungsanstalt eigentlich nicht gut eingreifen könne. Die Bedeutung der Grundlagenforschung wäre ihm nicht fremd, aber man könne sich auch vorstellen: "Wenn der Oberregierungsrat wissenschaftlich etwas taugt, könnte er einen in der anderen Besoldungs- oder Titelordnung stehenden Direktor durch Leistung glatt überspielen". Auch Prof. Hagmeier konnte sich nicht mit den Vorstellungen von Prof. Ankel befreunden. Er wünschte wohl mehr Selbständigkeit innerhalb der Bundesforschungsanstalt für Fischerei oder gar eine Trennung von ihr, aber nicht von dem zuständigen Ministerium.

\section{Die Wiedergewinnung von Helgoland}

Nach der Vertreibung der Helgoländer und der Beschlagnahme der Insel durch Großbritannien gab es immer neue Bemühungen auf vielen Ebenen um eine Wiederbesiedlung Helgolands. Schleswig-Holstein hatte schon 1946 die Militärregierung gebeten, Helgoland zurückzugeben, und 1947, die Insel nicht zu sprengen, aber beides blieb vergeblich. Am 18. 4. 1947 erfolgte die Sprengung der Insel. Ab 1949 gab es Helgoland-Debatten im Bundestag und im Britischen Unterhaus, und im Mai 1950 konnte man offenbar so hoffnungsvoll sein, daß der Schleswig-Holsteinische Landtag beschloß, den Wiederaufbau Helgolands vorzuplanen. Besonders eindrucksvoll für die Öffentlichkeit war die "Aktion Helgoland“ zum Jahreswechsel 1950/51, die "friedliche Invasion" der Studenten Georg von Hatzfeld und René Leudesdorff, politisch unterstützt durch den einflußreichen Prinzen zu Löwenstein (Leudesdorff, 1987).

Im Februar 1951 gab es schließlich eine verbindliche Zusage der britischen Regierung für die Freigabe, und in diesem Jahr wurden erstmalig Besuche auf Helgoland im Zusammenhang mit der Aufbauplanung erlaubt. Auch folgenden Mitgliedern der BAH 
wurde vom Land Commissioner Schleswig-Holstein gestattet, am 2. Juni 1951 in Ausübung ihres Dienstes die Insel zu betreten: Kapitän Jacob Holtmann, Steuermann Tönnies Hornsmann, Maschinist Peter Singer, Matrose Werner Krühs als Besatzung der "Uthörn" und als Mitfahrende: Prof. Hagmeier, Dr. Peter Kornmann, Peter Krüss und Andreas Holtmann. Bei Sonnenaufgang wurde die Insel angelaufen. Auf dem alten "Flakturm" wehte die Europaflagge, und Helgoländer Hummerfischer waren im Hafen, deren Auslaufen für die Wochenschau aufgenommen wurde. Die Biologen führten eine botanische Exkursion mit dem Beiboot durch, setzten von der "Uthörn" die Dredge ein und gingen nachmittags auf Felswattexkursion. Die Besichtigung der Ruine der Biologischen Anstalt am Nachmittag ergab "Aquariumsteil: Keller und zwei Geschosse noch brauchbar, Mittelteil und Bibliotheksteil weitgehend zerstört" (Quelle: handschriftliche Aufzeichnungen von Prof. Hagmeier). Landrat Schinkel vom Kreis Pinneberg kam an Bord der „Uthörn“, und auch Herr von Hatzfeld war auf der Insel. Auf der Rückfahrt wurde noch Plankton gefischt, gekurrt und auf dem Amphioxusgrund gedredgt. Man spürt aus den nüchternen Aufzeichnungen von Prof. Hagmeier die Begeisterung über den Formenreichtum der Flora und Fauna bei Helgoland.

Am 1. 12. 1951 wurde durch die Kreisverwaltung Pinneberg ein Ideenwettbewerb "zur Erlangung von Entwürfen für den Wiederaufbau der Insel" ausgeschrieben (Anonymus, 1951). "Die Biologische Anstalt", so heißt es darin, "war und soll wieder ein repräsentatives Bauwerk im allgemeinen städtebaulichen Bild darstellen." Aber: "Die geschlossene Bauform der alten Anstalt paßte sich in das städtebauliche Bild des früheren Helgoland nicht gerade günstig ein", und man sollte eine stärkere Gliederung des Baukörpers, eine funktionale Trennung oder gar einen anderen günstigen Standort einplanen. Dazu meinte Prof. Hagmeier allerdings, daß die alte Lage des Instituts am offenen Nordoststrand des Unterlandes im Interesse der Forschung und des Betriebes beibehalten werden müsse.

Am 28. 2. $1952 \mathrm{gab}$ es eine Feierstunde der BAH in List, um den Wiederbeginn der Arbeit auf Helgoland selbst einzuleiten. Dabei wurde des ersten Direktors des Instituts, Prof. Friedrich Heincke, gedacht, der am 6. Januar 1952100 Jahre alt geworden wäre und dessen Arbeiten den Grund für die Fischereibiologie gelegt hatten. Am 1. März 1952 übernahm nach nächtlichen Freudenfeuern der Ministerpräsident von Schleswig-Holstein, Friedrich-Wilhelm Lübke, Helgoland wieder in die deutsche Verwaltung. Unter den vielen Schiffen, die zu dieser Feier gekommen waren, befand sich auch die "Uthörn" der Biologischen Anstalt. Sie brachte die beiden ersten wieder auf der Insel stationierten Mitarbeiter Reimer Rickmers und Andreas Holtmann, der später durch Heinrich Herzog abgelöst wurde. Die erste Liste der Daten über Wassertemperatur und Salzgehalt beginnt am 3.3.1952. Hinzu kamen tägliche Planktonfänge, die Beschaffung von Pflanzen und Tieren für Versand sowie Versuche und die Fortführung der Fangstatistik des Hummers. Es zeigte sich, daß sich der Hummerbestand entgegen den Beobachtungen nach dem 1. Weltkrieg 1952 nicht erholt hatte. Zur Inselübernahme waren auch Prof. Hagmeier und Peter Krüß auf die Insel gekommen.

Für Peter Krüß (Abb. 4) war der 1. März 1952 der erste Tag im Ruhestand nach nahezu 54jähriger Dienstzeit. Er hatte als Vierzehnjähriger Ostern 1898 begonnen, bei Prof. Ehrenbaum Fischeier und Fischlarven aus dem Plankton auszusuchen, im Büro zu arbeiten und bei Prof. Kuckuck die Meeresalgen kennenzulernen. Algenkunde und Botanik waren seitdem zu seiner Lieblingsbeschäftigung geworden. Als Leiter des Aquari- 


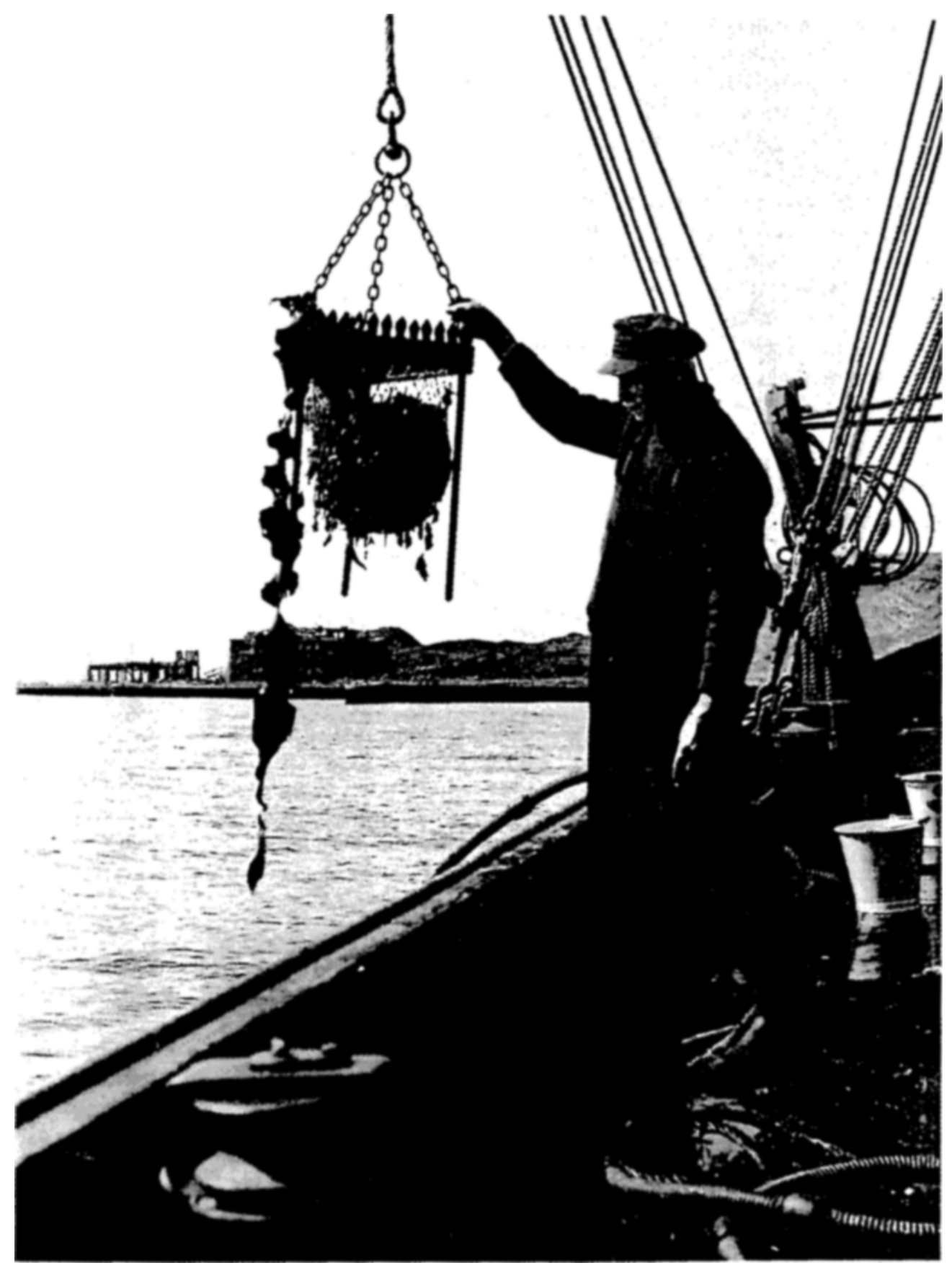

Abb. 7. Fischmejster und Kapitän Jacob Holtmann an Bord der "Uthörn" mit einer botanischen Dredge auf Helgoland-Reede, im Hintergrund die Ruinen von Kurhaus (links) und Biologischer Anstalt, etwa 1952. Photo: Andreas Holtmann. 
ums gelang es ihm später, die Becken so einzurichten, daß sie sich den Besuchern als lebensvolle und ästhetisch schöne Ausschnitte aus der Umgebung Helgolands darboten. Im Zweiten Weltkrieg und danach war Peter Krüß für den Direktor wieder eine wertvolle Hilfe im Büro. Im April 1948 erhielt Peter Krüß vorn damals zuständigen schleswig-holsteinischen Minister für Volksbildung eine schlichte Urkunde für 50jährige, mustergültige und treue Dienste. Nach seiner Pensionierung konnte Peter Krüß als Mitglied im Helgoland-Ausschuß für den Wiederaufbau seiner Heimatinsel weiterarbeiten.

Eine ähnlich lange und fruchtbare Dienstzeit an der BAH erlebte Jacob Holtmann (Abb. 7). Er begann bei der BAH am 1.4.1902 als Präparatorlehrling. Der junge Helgoländer mußte sich gleich auf vielen, weiten Reisen bis in die Barentssee auf dem Reichsforschungsdampfer "Poseidon" mit Planktonfängen und mit Messungen, Altersbestimmungen sowie Markierungen von Fischen beschäftigen. An Land kam zu den Laboruntersuchungen die Arbeit im Schauaquarium, dessen Verwalter er später wurde. Im Jahr 1921 wurde Jacob Holtmann Nachfolger des Fischereisachverständigen Uwe Jens Lornsen. Die Fischer kamen zu ihm, um sich bestätigen zu lassen, daß ihre Hummer bei Helgoland gefangen waren und somit zollfrei ins Deutsche Reich eingeführt werden durften. Jacob Holtmann wurde Kapitän der Forschungsschiffe "Augusta“. "Makrele" und "Uthörn“ und führte sie mit Umsicht und Erfolg für Wissenschaft und Unterricht.

Leider konnte Dr. Clemens Künne, der Kustos für Zoologie an der BAH seit 1941, den Wiederbeginn auf Helgoland nicht mehr erleben. Er starb im September 1951 kurz vor seinem fünfzigsten Geburtstag an einem Lungenleiden. Seine Mitarbeiter vermißten ihn sehr als freundlichen und hilfreichen Kollegen und hätten gerne sein reiches Wissen über das Zooplankton weiter genutzt, das er durch viele Forschungsfahrten und genaue Analysen gewonnen und als ausgezeichneter Lehrer in den Kursen vermittelt hatte (Bückmann, 1952).

Zum 65. Geburtstag von Prof. Hagmeier am 17.7.1951 und zur Vollendung von 40 Dienstjahren bei der BAH sind die Unterschriften der Mitarbeiter erhalten (Abb. 8). Es kamen Glückwünsche und Anerkennung vom Bundesminister für Ernährung, Landwirtschaft und Forsten, Prof. Niklas, und seinem Ministerialdirektor Dr. Tietmann. Die Dienstzeit von Prof. Hagmeier wurde verlängert, damit er weiter für die Planung des Aufbaus auf Helgoland zur Verfügung stand. Prof. Hagmeier leitete die BAH bis 1953, bis zu seinem 67. Lebensjahr. Bei der Benennung eines Nachfolgers gab es Schwierigkeiten. Prof. Kändler (Kiel) war zunächst bereit und schätzte sich glücklich, an seine frühere Wirkungsstätte als Nachfolger seines Mentors Arthur Hagmeier zurückkehren zu können, sagte dann aber im November 1952 ab. Im März 1953 war schließlich Prof. Adolf Bückmann, der gerade als Nachfolger von Prof. Willer auf das Ordinariat für Fischereibiologie in Hamburg berufen worden war, damit einverstanden, die BAH "ehrenamtlich" zu leiten. Damit hatte das Ministerium einen Wissenschaftler gewonnen, der die Anstalt schon seit 1923 als Assistent der Deutschen Wissenschaftlichen Kommission für Meeresforschung kannte und der seine Erfahrungen und seine Kräfte zum Wohle des Instituts einbringen sollte. Für die Verabschiedungsfeier von Prof. Hagmeier am 7. Mai 1953 in der Universität Hamburg kam die offizielle Benachrichtigung so spät in List an, daß Prof. Hagmeier nicht zu der Feier kam, in der er gemeinsam mit den Professoren Lücke und Schnakenbeck verabschiedet werden sollte. Prof. Hagmeier zog mit seiner Familie von Sylt nach Kiel, wo sein Sohn Erik Meereskunde studierte. Prof. Hagmeier starb 1957 in Kiel im Alter von 71 Jahren. 


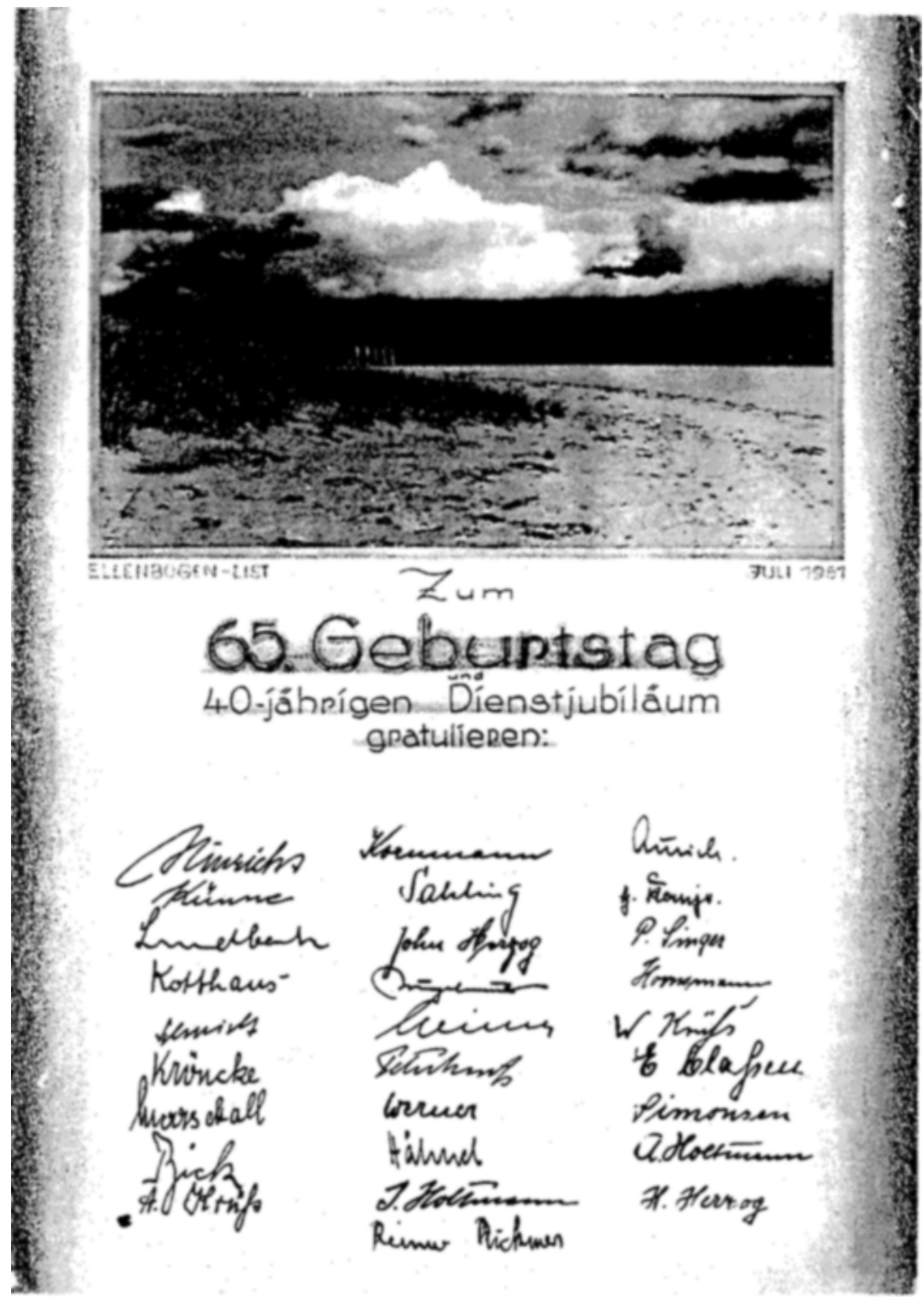

Abb. 8. Gratulation für Prof. Hagmeier zum 40jährigen Dienstjubiläum am 11.7. und zum 65. Geburtstag am 17.7.1951. Gestaltung: Dr. Erich Ziegelmeier. Unterschriften der Mitarbeiter. John Hinrichs (Oberpräparator), Dr. Clemens Künne, Dr. Johannes Lundbeck, Dr. Adolf Kotthaus, Dr. Ulrich Schmidt, Herr Kröncke, Jürgen Marschall (techn. Assistent und Zeichner), Herr Bick (Laborant), Anna Krüß (Hauspersonal), Dr. Peter Kormmann, Paul-Heinz Sahling (techn. Assistent, Botanik), John Herzog (Bootsführer), Dr. Erich Ziegelmeier, Erich Windt (Verwaltungsleiter), Peter Krüß, Dr. Bernhard Werner, Frau Hähnel (Bibliothek), Jacob Holtmann (Kapitän und Fischmeister), R. Rickmers (wissensch. Fischer), Dr. Horst Aurich, Hinrich Kanje (wissensch. Fischer), Peter Singer (Maschinist), Tönnies Hornsmann (Steuermann), Werner Krühs (Matrose), E. Claßen (Hauspersonal), Max Simonsen (Werkstatt und Fahrer), Andreas Holtmann (techn. Assistent), Heinz Herzog (techn. Assistent). 


\section{Professor Bückmann und die „Kopfstation“ der BAH}

Mit Prof. Bückmann (Abb. 9) wurde ein Wissenschaftler als Direktor der BAH ernannt, dessen Begeisterung für die Meeresbiologie schon während seiner Studienzeit in Hamburg geweckt wurde. Eine ausführliche Lebensbeschreibung, die Liste der Veröffentlichungen und die seiner Diplomanden und Doktoranden wurde von Schmidt (1970) publiziert. Adolf Bückmann promovierte 1923 bei Prof. Hans Lohmann in Hamburg über

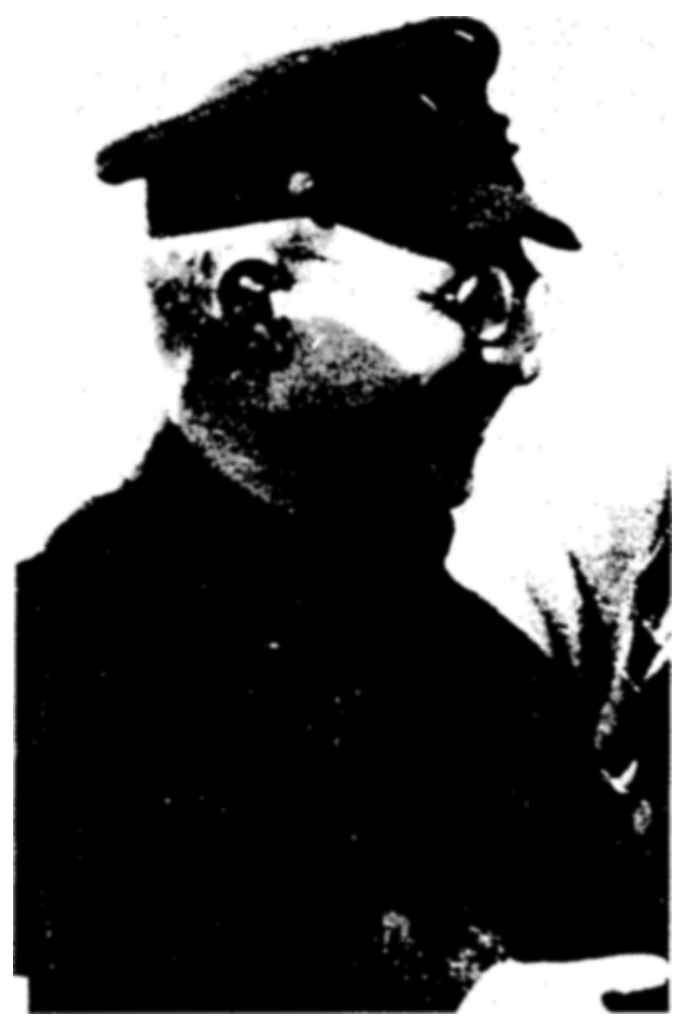

Abb. 9. Prof. Bückmann 1929 an Bord des RFD "Poseidon ". Photo: Archiv der BAH.

Appendicularien, eine Gruppe, die er auch für die "Tierwelt der Nord- und Ostsee " bearbeitete und die ihn nach seiner Emeritierung wieder beschäftigte. Am 16. Mai 1923 wurde er Assistent der Deutschen Wissenschaftlichen Kommission für Meeresforschung auf Helgoland, nachdem Dr. Werner Schnakenbeck von dort an das Fischereibiologische Institut in Hamburg gegangen war, und untersuchte zusammen mit Prof. Friedrich Heincke die Auswirkungen der Fischerei auf den Schollenbestand der Nordsee. Adolf Bückmann nahm an der 47. „Poseidon"-Fahrt teil und an sehr vielen weiteren Forschungsfahrten auf der "Poseidon", "Augusta“, "Makrele“, auf Fischereischutzbooten und Fischdampfern, später auf "Gauss", "Anton Dohr" " und "Meteor". Neben der Erledigung des großen Arbeitspensums auf derartigen Reisen sorgte er auch für den ge- 
selligen Teil, auf der alten "Gauss" im edlen Wettstreit mit Dr. Joachim Joseph, und für humoristisch-poetisch-philosophische Beiträge in den Gästebüchern der Forschungsschiffe. Aus seiner Feder stammen zahlreiche, grundlegende Arbeiten über das Wachstum der Fischbestände, die Befischung und den Ertrag sowie über fischereibiologische Untersuchungsmethodik.

Prof. Bückmann war durch seine Lehrverpflichtung und die Leitung des Instituts für Fischereiwissenschaft der Universität an Hamburg gebunden, und er setzte sich dafür ein, daß auch die BAH in Hamburg vertreten war. Im Jahr 1954 wurde eine "Kopfstation" der BAH in Hamburg eingerichtet, zunächst mit dem Direktor und einem Teil der Verwaltung im Paulsenhaus, Neuer Wall 72, wo die meisten Institute der Bundesforschungsanstalt für Fischerei untergekommen waren, ab Anfang 1955 in der angemieteten Villa Elbchaussee 199 (Abb. 10). Dort konnten auch experimentelle Arbeiten über die Rhythmik von Ruhe und Aktivität von Fischen aufgenommen werden. Zunächst wurde das Verhalten von Aalen geprüft, was für Nahrungsaufnahme und Fang bedeutsam ist. Die neu geschaffene Abteilung Tierphysiologie kam hinzu, und zwar unter der Leitung von Prof. Friedrich Krüger, der von der Universität Münster als außerplanmäßiger Professor nach Hamburg wechselte und ab 1.4.1956 zur BAH gehörte. Sein wissenschaftlicher Assistent war zunächst Dr. Jakovlev. Prof. Bückmann etablierte auch die Abteilung Meeresmikrobiologie an der BAH und sandte im Jahr 1959 Dr. Wilfried Gunkel als ersten Meeresmikrobiologen der BAH zur Ausbildung in die USA zu Prof. ZoBell in La Jolla, Kalifornien, und zu Prof. Carl H. Oppenheimer in Port Aransas, Texas.

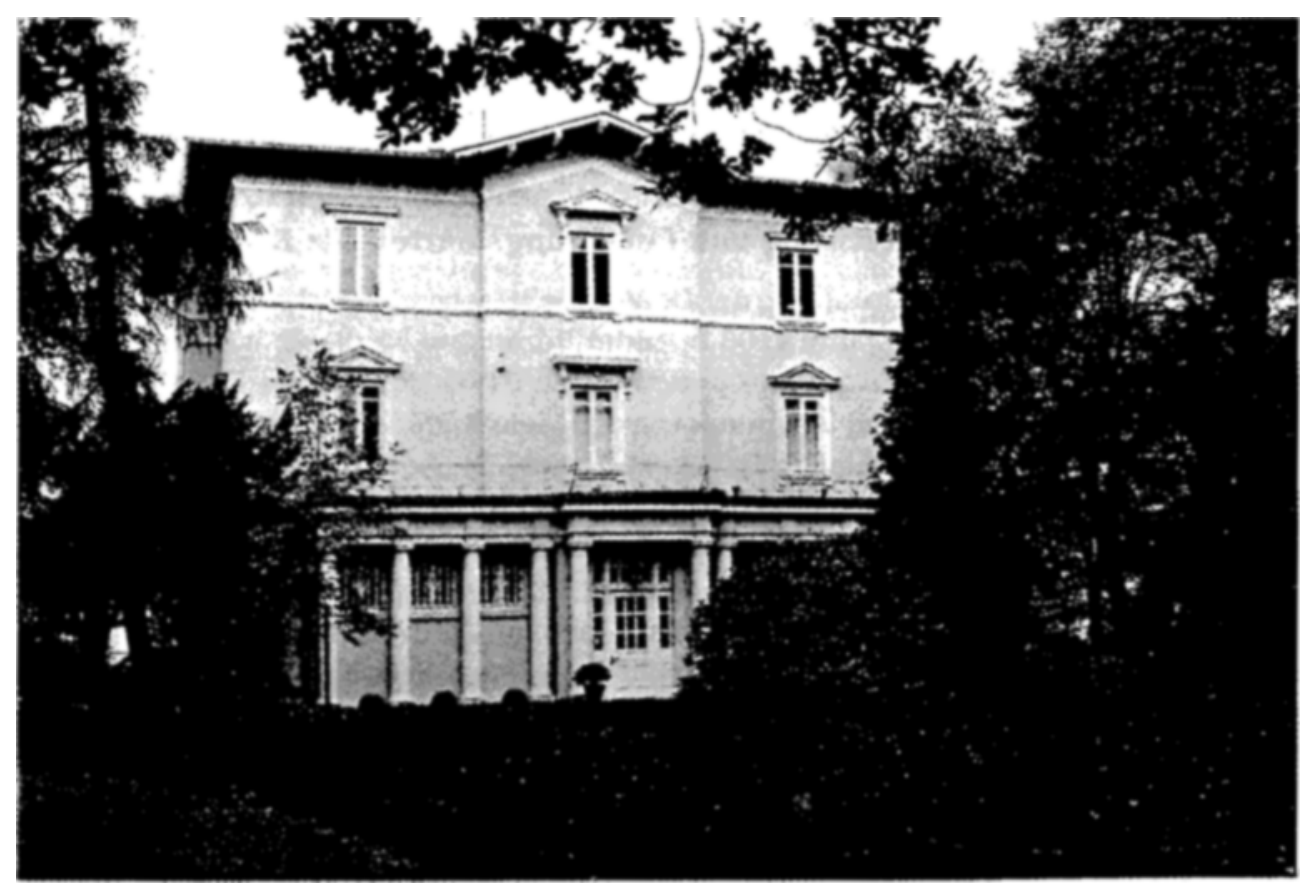

Abb. 10. Das Gebäude Elbchaussee 199, heutiger Zustand. Photo: Archiv der BAH. 
Verbindungen mit Hamburg waren übrigens nicht neu in der Geschichte der BAH. Nach 18jähriger Arbeit auf Helgoland wurde Dr. Ehrenbaum 1910 zum Leiter der neuen Fischereibiologischen Abteilung am Naturhistorischen Museum in Hamburg berufen. Während des Ersten Weltkriegs gab es in dieser Abteilung und im Museum Platz für die von Helgoland evakuierten Mitarbeiter der BAH. Im Jahr 1921 wechselte Dr. A. Wulff von Hamburg nach Helgoland, 1923 kam Dr. Schnakenbeck von Helgoland nach Hamburg und wurde 1932 Abteilungsdirektor und Professor. Aus dieser Abteilung entstand nach 1945 das Institut für Hochseefischerei der Bundesforschungsanstalt für Fischerei, deren Verwaltung, wie oben erwähnt, von dem Helgoländer Henry Bartz geleitet wurde.

Die Bemühungen von Prof. Hagmeier um eine möglichst selbständige Stellung der BAH wurden von Prof. Bückmann während seiner gesamten Amtszeit fortgesetzt. Dabei erfuhr er wirksame Unterstützung durch die Deutsche Forschungsgemeinschaft (DFG), die in einer Besprechung auf ministerieller Ebene im Januar 1954 und in ihrer "Denkschrift über den Wiederaufbau der Biologischen Anstalt für Meeresforschung in Helgoland" im März 1954 die Wünsche der deutschen Wissenschaft in Bezug auf die Zukunft der BAH deutlich machte. Gemäß der Denkschrift der DFG sollten die idealen Bedingungen auf Helgoland für die Beobachtung und Beschaffung von Meerespflanzen und Meerestieren durch einen festen Mitarbeiterstab und durch zeitweilige Gäste für die Grundlagenforschung genutzt werden. Außerdem wäre die Station besonders wichtig zur Ausbildung des Nachwuchses für moderne anatomische, physiologische und biochemische Arbeitsrichtungen. In der Denkschrift der DFG wurden die Herauslösung der $\mathrm{BAH}$ aus dem Haushalt der Bundesforschungsanstalt für Fischerei empfohlen, die geplante Berufung eines Beirats für die BAH durch das Ernährungsministerium begrüßt sowie die Einrichtung von zahlreichen, großen Räumen für Gastforscher und Kurse auf Helgoland gefordert.

\section{Mitarbeit der BAH in der Deutschen Wissenschaftlichen Kommission für Meeresforschung und Forschungsfahrten der BAH}

Die BAH stand seit der Gründung der Deutschen Wissenschaftlichen Kommission für Meeresforschung (DWK) im Jahre 1900 in enger Beziehung zu dieser Institution (Bahr, 1962). Prof. Friedrich Heincke gehörte zu den ersten DWK-Mitgliedern. DWK-Assistenten arbeiteten an der $\mathrm{BAH}$, und von ihnen wurden viele später als planmäßige Mitarbeiter der BAH übernommen, nämlich Dr. Horst Aurich, Dr. Adolf Bückmann, Dr. Arthur Hagmeier, Dr. Helmuth Hertling, Dr. Clemens Künne, Dr. Johannes Lundbeck, Dr. Wilhelm Mielck, Dr. Ulrich Schmidt, Dr. Hugo Weigold und Dr. Alfred Wulff. Im Jahr 1920 wurde für den Zweiten Vorsitzenden der DWK die Bezeichnung "Wissenschaftlicher Vorsitzender" eingeführt (Erster Vorsitzender war ein Ministerialbeamter), und das Ernährungsministerium berief Prof. Heincke in dieses Amt. Sein Nachfolger wurde 1929 Prof. Ehrenbaum, und diesem folgte im Jahr 1935 Prof. Hagmeier. Die DWK war außerdem für die Organisation und Finanzierung der "Poseidon"-Fahrten zuständig und sorgte für Verbindung und Abstimmung mit dem Internationalen Rat für Meeresforschung. Dr. Julius Claussen als Vorsitzender der DWK und Dr. Meseck als geschäftsführender Sekretär setzten sich nach dem Zweiten Weltkrieg für das Fortbestehen der DWK ein. Die DWK wurde zunächst in den Haushaltsplan des Zentralamtes für 
Ernährung und Landwirtschaft in der Britischen Besatzungszone aufgenommen und kam schließlich in den Zuständigkeitsbereich des Bundesernährungsministeriums. Für Assistentenstellen waren erst ab 1956 wieder Mittel vorhanden. Zu den ersten wissenschaftlichen Nachkriegs-Assistenten gehörte Dr. Gotthilf Hempel an der BAH. Vorher war bereits die Herausgabe der "Berichte der Deutschen Wissenschaftlichen Kommission für Meresforschung" möglich, die ab 1949 mit Band 11 wieder erscheinen konnten.

Im Jahr 1948 fand die erste Sitzung der DWK nach dem Kriege statt, mit Dr. Lundbeck als Vertreter der BAH. Am 1.11. 1952 konnte die deutsche Mitgliedschaft im Internationalen Rat für Meeresforschung (ICES) erneuert werden. In den "Annales Biologiques" für 1952 erschienen wieder ICES-Arbeitsberichte über die deutschen Untersuchungen, aus der BAH von Dr. Aurich, Dr. Kotthaus, Dr. Lundbeck und Dr. Erich Ziegelmeier. Eine Mitarbeit der deutschen Meeresforscher an größeren internationalen Untersuchungen wurde wieder möglich und auch verlangt, wie etwa bei der regelmäßigen Kontrolle der Fischbestände. Damit konnten sich die deutschen Wissenschaftler wieder an der Abfassung von Empfehlungen und an Beschlüssen beteiligen, die für Fischereikonventionen ausschlaggebend waren. Prof. Bückmann hatte schon seit 1928 an den Konferenzen des ICES teilgenommen. Im Jahr 1934 wurde er Sekretär der DWK, 1936 Mitglied und 1953 ihr Wissenschaftlicher Vorsitzender. Im Jahr 1959 gab Prof. Bückmann mit Rücksicht auf seine sonstigen vielfältigen Aufgaben den Vorsitz der DWK an Prof. Günter Dietrich $a b$, blieb aber ein sehr engagierter Mitarbeiter und Diskussionspartner der DWK und ein vieigefragter Fachmann. Wissenschaftler der BAH hatten stets Anteil an der Arbeit der DWK, wie Dr. Aurich, Dr. Max Gillbricht, Prof. Otto Kinne, Dr. Kotthaus, Dr. Harald Rosenthal und Dr. Friedrich-Wilhelm Tesch. Zur Zeit gehört Dr. Hein v. Westernhagen der DWK an.

Großen Auftrieb für die Arbeit der DWK und für die deutsche Beteiligung an internationalen Aufgaben gab im Jahr 1955 die Indienststellung des Forschungsschiffes "Anton Dohrn" (Abb. 11). Prof. Bückmann betonte beim Stapellauf in Cuxhaven am 16. 8. 1954 die Wichtigkeit einer Überwachung der Nutzfischbestände, wobei Forschungsschiffe das notwendige Material liefern. Er erinnerte an die vielen Fahrten der Meereskundler mit dem Reichsforschungsdampfer "Poseidon" im Zeitraum 1902-1938 sowie an die wertvollen Ergebnisse dieser Reisen für Wissenschaft und Praxis. In den von der Bundesforschungsanstalt für Fischerei herausgegebenen "Wissenschaftlichen Informationen für die Fischereipraxis" (später "Informationen für die Fischwirtschaft") sind Einzelheiten von den Fahrten der "Anton Dohrn" nachzulesen, von Teilnehmern, Arbeiten, Wind und Wetter sowie von der Begeisterung über das gute und praktische Schiff.

Auf der Erprobungsfahrt der "Anton Dohrn" im Februar 1955, mit einer Bewährungsprobe bei vollem Sturm, war Prof. Bückmann der Fahrtleiter. Fahrtteilnehmer seitens der BAH waren Dr. Reimer als Bordbiologe sowie Dr. Aurich, Dr. Ziegelmeier und der Kapitän der "Uthörn", Tönnies Hornsmann, die Netze und Bodengreifer erprobten. Im März 1955 gab es synoptische Fahrten von "Anton Dohrn" , "Gauss" und "Uthörn" in der südlichen Nordsee zur Untersuchung von Fischbrut, Plankton und Hydrographie mit Prof. Bückmann, Dr. Aurich, Dr. Gillbricht, Dr. Ziegelmeier, Heinrich Herzog und Andreas Holtmann. Damit wurden die 1928 aufgenommenen Arbeiten der BAH fortgesetzt. Es ging um den vermuteten Zusammenhang zwischen dem Erfolg des Laichens, also der Stärke der Nachwuchsjahrgänge, mit den Eigenschaften und Bewegungen des Wassers. In den Jahren 1952 und 1953 hatte man darüber nur mit der "Gauss" arbeiten können. 


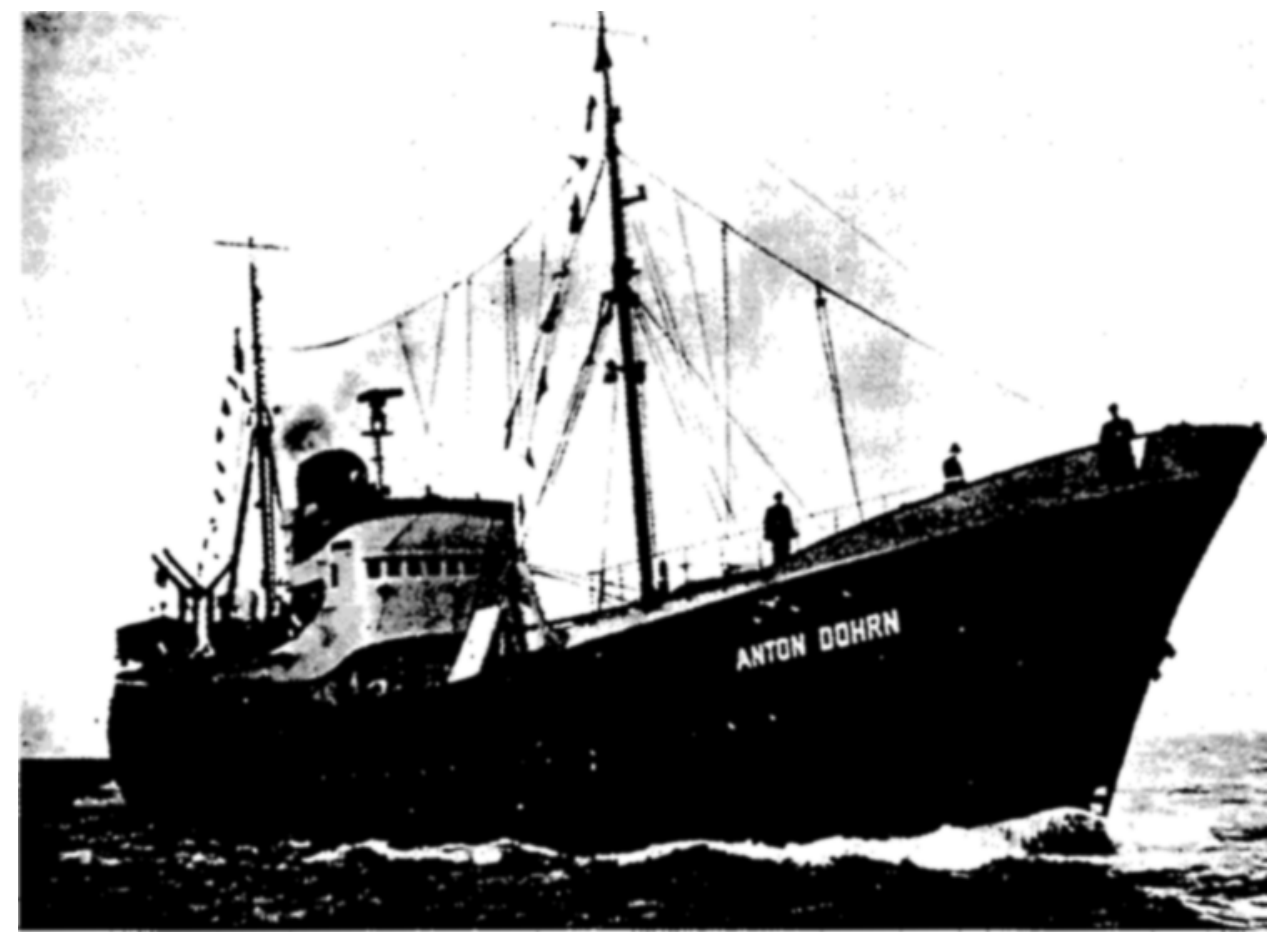

Abb. 11. „Anton Dohrn“, Länge 62,3 m, 999 BRT. Fischerei-Forschungsschiff 1955-1972. Photo: Archivbild der Bundesforschungsanstalt für Fischerei.

Im März 1955 dauerte der Winter lange. Die Wassertemperaturen waren niedrig, Laichen und Schlüpfen wurden verzögert. Erschwerend war, daß die "Uthörn “ Anfang März noch vom Eis in der Elbe festgehalten wurde.

Die folgende Reise der "Anton Dohrn" nach Island diente einer Erprobung der fischereilichen Ausrüstung. Die Fänge wurden für die laufenden Köhler- und Rotbarschuntersuchungen von Dr. Schmidt und Dr. Kotthaus ausgewertet. Die erste längere Reise der "Anton Dohrn“ ging im Sommer 1955 in die Irminger See. Eine Bank, mit reichem Rotbarschvorkommen, die zuvor "amtlich" nicht bekannt war, wohl aber den Fischern, wurde "Anton-Dohrn-Bank" benannt. Der Fahrtleiter Dr. Kotthaus bearbeitete zusammen mit Dr. Gerhard Krefft die Fischfauna. Dr. Aurich und Dr. Gillbricht befaßten sich mit dem Mikroplankton sowie seinen Beziehungen zu den Trübungsmessungen. Dabei wurde eine Zone parallel zur grönländischen Küste gefunden, in der die "Schaumalge" Phaeocystis vorherrschte. Dr. Ziegelmeier untersuchte die Bodengreiferproben und die Wirbellosen-Beifänge aus den Trawls.

Die "Anton Dohrn" wurde in der Folge mit Dr. Schmidt als Fahrtleiter regelmäßig im Winter zu den Untersuchungen der Köhlerbestände zwischen Norwegen und Island eingesetzt. Im November/Dezember 1958 ging es sogar bis in die Barentssee. Auf diesen Reisen wurden wichtige internationale Verbindungen geknüpft, gemeinsame Untersuchungen mit norwegischen Kollegen ausgeführt und unter harten Witterungsbedingun- 
gen Geräte getestet sowie Mitarbeiter geschult. Dr. Kotthaus fuhr mit der "Anton Dohrn" in jedem Frühjahr zur Untersuchung der Rotbarschvorkommen in den Nordatlantik zwischen Färöern und Island und im Juni/Juli zur Erforschung der Grundfischbestände in den südlichen Teil der Nordsee. Damit wurden die Untersuchungen der "Uthörn", die sich in den Jahren 1948-1955 auf die Deutsche Bucht beschränken mußten, wesentlich ergänzt. Es ging dabei auch um die Ernährung von Kliesche und Scholle. Hälterungen im Aquarium erlaubten Vergleiche über die Zeit der Nahrungsaufnahme sowie über die Geschwindigkeit der Verdauung.

Im Juni/Juli 1956 leitete Dr. Aurich die "Schollenreise” der „Anton Dohrn“. Er richtete bei Helgoland zur Untersuchung der Tagesperiodizität der Ernährung eine Dauerstation ein. Planktonfänge auf dieser Fahrt bestätigten das erstmals in den Nachkriegsjahren festgestellte, ausgedehnte und intensive Laichen von Sardine und Sardelle in den küstennahen Gebieten vor den west- und ostfriesischen Inseln sowie für die Sardelle außerdem noch vor der nordfriesischen Küste. Im Rahmen der norwegisch-deutschen Seelachsarbeiten wurde die "Anton Dohrn" unter Dr. Schmidt im November/Dezember 1956 und mit Fortsetzungen im Februar/März sowie November/Dezember 1957 eingesetzt. Dr. Kotthaus untersuchte auf einer Fahrt mit der "Anton Dohrn“ im März/April 1957 die Nutzfische in der Dänemarkstraße und im Juni/Juli 1957 in der südlichen Nordsee, wobei Vergleichsfänge mit der "Uthörn" ausgeführt wurden.

Im März/April und August/September 1958 war die BAH mit mehreren Mitarbeitern an den Untersuchungen im Rahmen des Internationalen Geophysikalischen Jahres beteiligt. Auf der "Anton Dohrn" war Prof. Bückmann Fahrtleiter der ersten Fahrt. Für das Phytoplankton war Dr. Gillbricht zuständig, für das Zooplankton waren es Dr. Schmidt und Dr. Ziegelmeier. Proben für Fischereibiologie und Benthos wurden von Jürgen Marschall und Martin Söhl genommen und für physiologisch-chemische Untersuchungen von Dr. Heinz Schaefer. Auf der "Gauss“ wurde das Phytoplankton von Dr. Aurich bearbeitet. Daraus ergaben sich Veröffentlichungen von Dr. Aurich über die Planktongemeinschaften verschiedener Wasserarten, von Prof. Bückmann mit den anderen Fahrtleitern (Prof. Dietrich, Dr. Joseph) über Programm und Verlauf der Fahrten, von Dr. Gillbricht über Phytoplankton und gelöste organische Substanz, von Dr. Kotthaus über den Rotbarsch und seine Larven, von Dr. Schaefer über Aminosäuren in der Rumpfmuskulatur des Rotbarsches und von Dr. Ziegelmeier über die Bodenfauna auf grönländischen und isländischen Schelfgebieten.

Im Januar 1959 fuhr die "Anton Dohrn" mit Dr. Hempel als Fahrtleiter in den Englischen Kanal. Diese Reise war ein Glied in der internationalen Kette von Forschungsfahrten, die von November bis März die Verteilung und Wanderungen der Heringslarven erfassen sollte. Es ging um die Frage, ob die Hauptmenge der Heringsbrut aus dem Englischen Kanal in der Deutschen Bucht aufwächst. Erstmalig wurde mit dem in den USA entwickelten "Hai" gearbeitet, einem quantitativen Planktonnetz, das vom fahrenden Schiff eingesetzt wird und gegen grobe See nicht empfindlich ist. Es wurde auch nach lohnenden Fangplätzen für die deutsche Heringsfischerei gesucht. Weiter konnten für das neue BAH-Aquarium Fische, große Krebse, Pilgermuscheln und ein Krake lebend nach Helgoland gebracht werden. Gleichzeitig begann Dr. Wilfried Gunkel, der Leiter der an der BAH neu eingerichteten Abteilung Mikrobiologie, an Bord der "Anton Dohrn" mit seiner Arbeit über die Verteilung der Bakterien in verschiedenen Wasserkörpern. 


\section{Wiederaufbau auf Helgoland, Mitarbeiter, Gäste und Kurse}

Im Jahr 1955 begann der Neubau von Institutsgebäude der BAH und Aquarium auf Helgoland (Abb. 12), nachdem der Baugrund nach Bomben und Minen durchsucht war. In den Helgoländer Wissenschaftlichen Meeresuntersuchungen wurde der Neubau beschrieben (Bückmann, 1959). Ein Nachbau des zerstörten Gebäudes hätte nicht in das durch einen Architektenwettbewerb bestimmte, völlig neugestaltete Ortsbild gepaßt. Im Vergleich zum alten Institutsgebäude wurde der ausgeführte Entwurf von Prof. G. Hassenpflug niedriger, länger und horizontal stärker gegliedert.

Das Aquarium wurde nur durch den Keller mit dem übrigen Institutsgebäude verbunden. Der Haupteingang sowie das offene Seehundsbecken sollten für einen ersten Blickkontakt der Besucher mit den Meeresbewohnern dienen. Die Verse aus Goethes "Faust" von der Giebelwand des ersten Aquariumsbaus wurden in der neuen Eingangshalle angebracht: "Alles ist aus dem Wasser entsprungen, alles wird durch das Wasser erhalten, Ozean gönn' uns dein ewiges Walten". Der Schauraum erinnerte sehr an das alte Aquarium, wurde jedoch von $170 \mathrm{~m}^{2}$ auf $210 \mathrm{~m}^{2}$ vergrößert. Hinter den Becken gab es jetzt wesentlich mehr Platz für die Pflege der Tiere und Pflanzen. Die Besucher fühlten sich wieder in die Helgoländer Unterwasserwelt versetzt. Der Natur nachgebildete Fels- und Sandböden mit ihren Bewohnern wurden gezeigt, und auch das bewährte "Arenabecken" wurde neu erbaut. Zur Schau gestellt waren wie früher auf einer ortsfremden Basalttribüne die farbprächtigen Seenelken und Seerosen. Das „Bootswrack“ im Knurrhahnbecken war wiedererstanden, und ebenso gab es, wie früher, die Reihe der Hummer verschiedenen Alters. Für die Wasserversorgung von Aquarium und Institut wurden große Tiefbehälter zum Sedimentieren der Trübstoffe sowie zwei Hochbehälter gebaut, die nicht mehr im Hause lagen, sondern, vor Temperaturschwankungen ge-

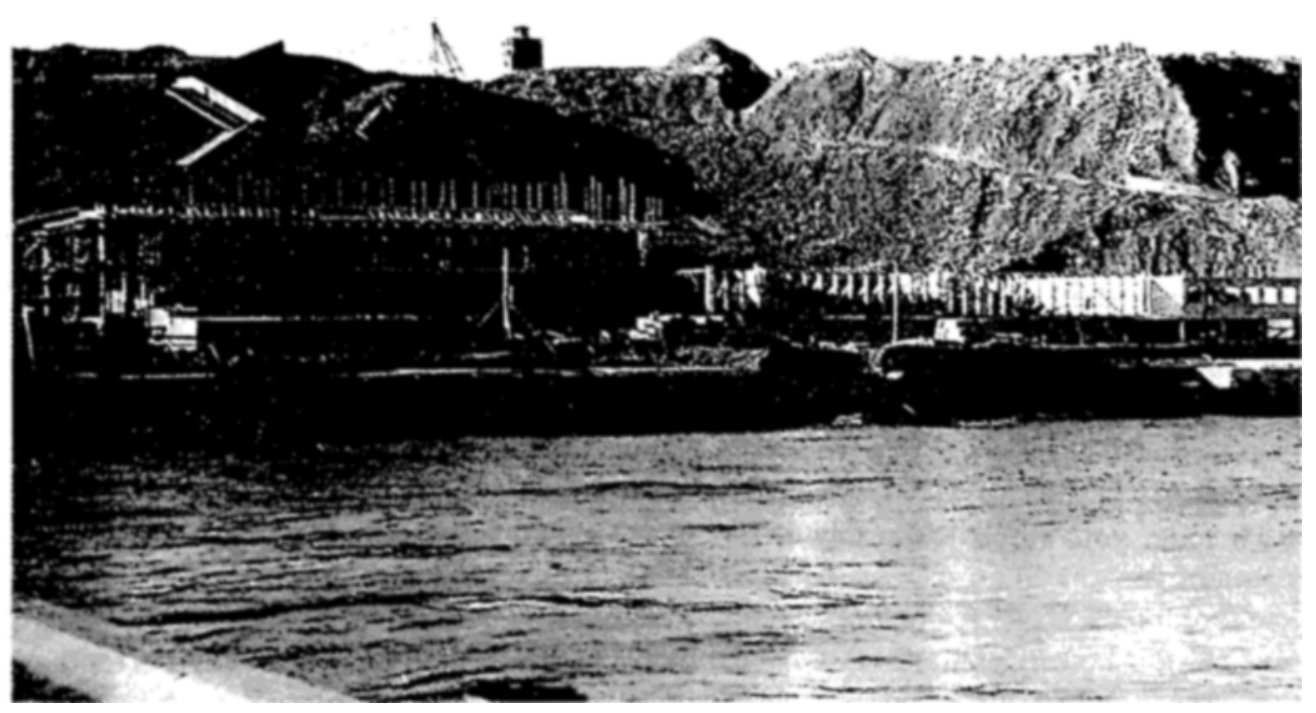

Abb. 12. Die neue BAH auf Helgoland 1956. Photo: Andreas Holtmann. 
schützt, in der Halde unter der Treppe zum Oberland. Im Keller befanden sich noch zahlreiche Aquarien für Versandmaterial und für die Versorgung von Gästen und Kursen sowie eine Reihe von Räumen zur Hälterung von Pflanzen und Tieren bei bestimmten Temperaturen.

Ein Flügel des Institutsgebäudes wurde für die auf Helgoland tätigen Mitarbeiter der BAH mit Laboratorien für Botanik, Zoologie, Physiologie, Mikrobiologie, Fischereibiologie und Planktonforschung gebaut. Im zweiten Teil des Gebäudes wurden Räume für Gastforscher sowie Kurse und im Keller Werkstätten sowie ein Isotopenlabor untergebracht. Zur Ausstattung der Gastforscherlabors stellte die Deutsche Forschungsgemeinschaft zahlreiche Geräte zur Verfügung. Für Lehrveranstaltungen waren ein Kurssaal für 30 Personen und ein „Exkursionssaal“ für 20 Personen vorgesehen, die bei Bedarf durch eine Schiebewand zu einem großen Raum verbunden werden konnten. Wegen zahlreicher Anmeldungen von Universitätskursen wurden die Räume aber meistens getrennt und parallel von Kursen genutzt. Eine Institutsbibliothek war zunächst nur in bescheidenem Maße auf Helgoland vorhanden.

Im Jahr 1959 gab es bei der BAH sechs Abteilungen und 12 Wissenschaftler: Zoologie: Prof. Bückmann, Dr. Werner, Dr. Ziegelmeier (Dr. Gotram Uhlig ab April 1960); Physiologie: Prof. Krüger, Dr. Henning Stieve; Botanik: Dr. Kornmann, Dr. Kesseler; Mikrobiologie: Dr. Gunkel; Planktonkunde: Dr. Aurich, Dr. Gillbricht; Fischereibiologie: Dr. Kotthaus, Dr. Hempel. Die BAH-Mitarbeiter Dr. Schmidt, S. Bick, R. Stündel und Räume der BAH in der Außenstelle am Fischmarkt in Bremerhaven wurden dort vom Institut für Seefischerei der Bundesforschungsanstalt für Fischerei (Hamburg) übernommen.

Anfang Mai 1959 waren Dr. Kornmann, Dr. Gillbricht, Dr. Kesseler, Erich-Hartwig Harms, John Herzog, Andreas Holtmann, Jacob Holtmann als Pensionär, Hinrich Kanje und Werner Krühs mit ihren Familien mit der "Uthörn" von List nach Helgoland gezogen. Nach der sehr bewegten Überfahrt erfolgte die Begrüßung der Umsiedler beim Einlaufen in den "Nord-Ost-Hafen" vor dem neuen Institutsgebäude durch Paul-Heinz Sahling und Dr. Gunkel.

Das Gästebuch hat die Besucher zur feierlichen Eröffnung der Station auf Helgoland am 19.6.1959 festgehalten. An erster Stelle steht Heinrich Lübke, Bundesminister für Ernährung, Landwirtschaft und Forsten, designierter Bundespräsident. Dr. Verwey, Direktor der Zoologischen Station in Den Helder und seit 1922 mit Helgoland verbunden, hielt den Festvortrag über Wanderungen von Meerestieren in der Nordsee, besonders über die Bewegungen und die Orientierung der Garnelen. Am Abend gab es ein frohes Fest an Bord der "Anton Dohrn" im Helgoländer Hafen.

Auch die Lehrlingsausbildung wurde in Helgoland fortgesetzt. Im noch unfertigen Aquarium begann Gerrit Sahling am 1.4.1959 als Lehrling und machte unter Andreas Holtmann und "Hinni" Kanje seine ersten Einrichtungsarbeiten.

Gastforscher sowie der erste Studentenkurs aus Tübingen folgten noch im gleichen Jahr, und alle waren beeindruckt von den neuen Labors. Auch das Gästehaus auf dem Oberland (Abb. 13) konnte schon im Herbst 1959 genutzt werden. Die erste Heimleiterin war Elisabeth Harms. Im Jahr 1960 gab es 11 Kurse oder Exkursionen mit 298 Teilnehmern, darunter zwei "anstaltseigene“ Kurse für Lehrer und Studenten im April sowie im August. Es kamen 32 Arbeitsplatzbeleger, darunter Gäste aus der Vorkriegszeit wie Prof. Kuhl vom Institut für Kinematische Zellforschung und junge Wissenschaftler, die später der BAH besonders nahe stehen sollten wie Dr. Jürgen Overbeck aus Potsdam und 


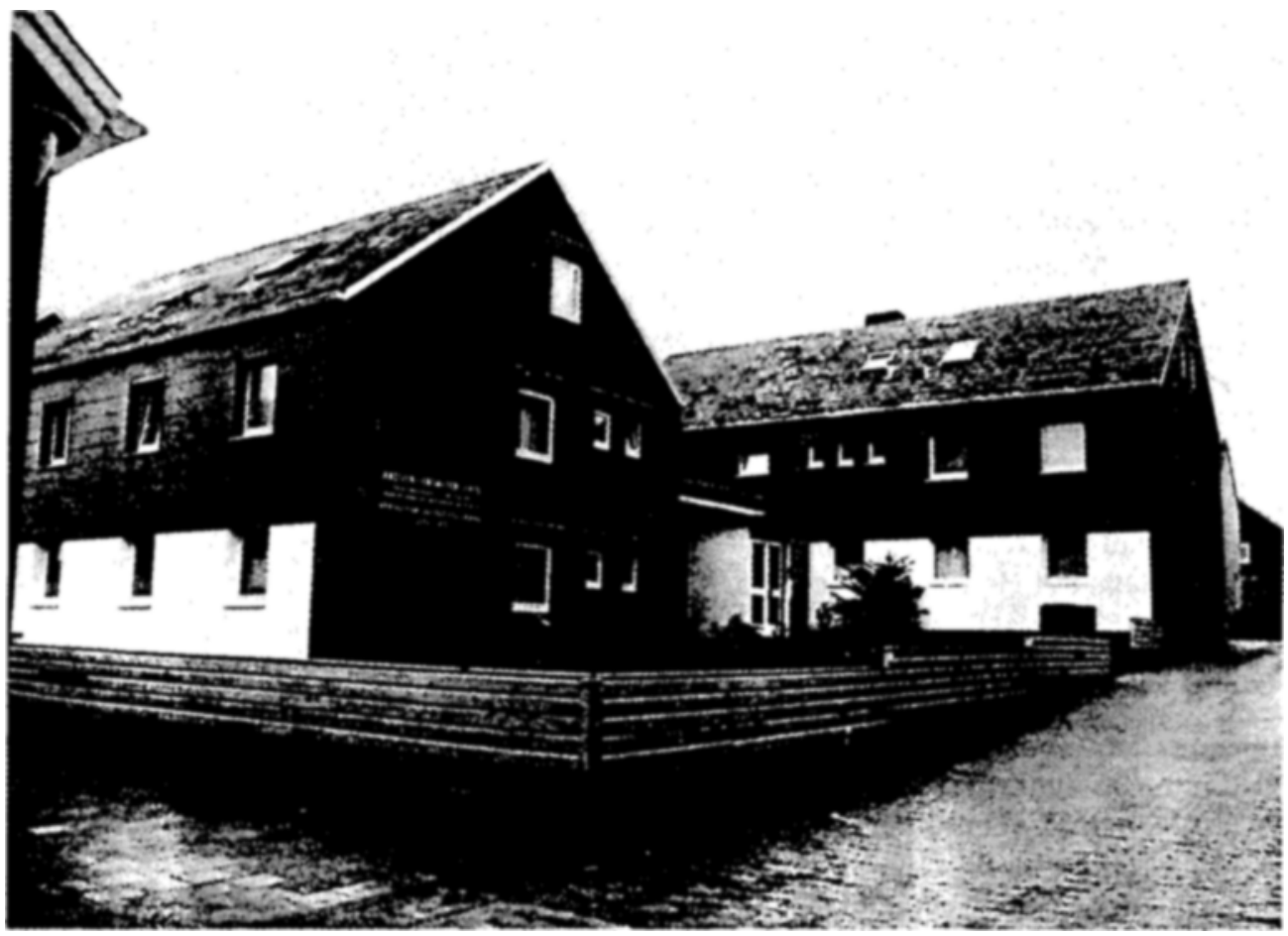

Abb. 13. Das Gästehaus der BAH auf Helgoland, Oberland. Erbaut 1959. Später „Arthur-HagmeierHaus". Photo: Paul-Heinz Sahling.

Dieter Mollenhauer, stud.rer.nat. aus Berlin, oder später dem BAH-Mitarbeiterstab angehörten wie Jürgen Flüchter, Diplombiologe aus Hamburg, weiter Klaus-Jürgen Götting, cand.rer.nat. aus Gießen und später Professor in Gießen. In der Liste des Verwaltungsleiters gibt es Vermerke zur Finanzierung der Gastforschungsaufenthalte. Man konnte über die Kultusverwaltungen der deutschen Länder, die Deutsche Forschungsgemeinschaft oder die Max-Planck-Gesellschaft kommen, die Arbeitsplätze pauschal gemietet hatten, oder man wurde als "Selbstzahler" geführt.

Im Juni 1960 gab es eine Tagung der Arbeitsgruppe für Benthos-Untersuchungen des Internationalen Rats für Meeresforschung unter dem Vorsitz von Prof. Gunnar Thorson (Kopenhagen), mit Prof. Otto Kinne als Gast aus Kanada, Dr. Harold Barnes von der Millport Marine Station, Dr. N. S. Jones aus Port Erin, Isle of Man, Dr. Frederik Beyer aus Oslo, Dr. Roland L. Wigley aus Woods Hole, Dr. Leon Birkett aus Lowestoft, Prof. J. M. Pérès aus Marseille, Dr. A. D. McIntyre sowie Dr. J. H. S. Blaxter aus Aberdeen, Dr. N. A. Holme aus Plymouth, Prof. den Hartog aus Leiden, Dr. Korringa aus Ijmuiden, Dr. A. R. Longhurst aus Freetown und Prof. Erik Dahl aus Lund. Die Gäste drückten ihre Begeisterung über Insel und Institut im Gästebuch aus.

Ebenfalls im Jahr 1960 fand auf Anregung von Prof. Schlieper (Kiel) das Erste Meeresbiologische Symposium auf Helgoland statt, zu dem Prof. Bückmann vorwiegend deutsche Kollegen in das neue Haus eingeladen hatte. Das primäre Ziel war eine ver- 
stärkte Zusammenarbeit der deutschen meeresbiologischen Institute in Kiel, Hamburg, Helgoland und Bremerhaven. Wieder findet sich im Gästebuch eine lange Liste bekannter Meereskundler, die gekommen waren, um über ihre Arbeiten zu berichten und Erfahrungen auszutauschen. Es ging vor allem um Tierwanderungen in der Nordsee und angrenzenden Meeresgebieten sowie um ökologische und physiologische Unterschiede innerhalb der in Nord- und Ostsee vorkommenden Arten. Die Gelegenheit zu Diskussionen, zu gegenseitiger Information und Absprache unter den Kollegen wurde dankbar begrüßt, und zum Schluß luden Prof. Dietrich und Prof. Schlieper zu einem nächsten Symposium 1961 nach Kiel ein. Vorträge und Diskussionen wurden noch nicht in einem Band gedruckt wie bei späteren Symposien, und in den "Helgoländer Wissenschaft. lichen Meeresuntersuchungen" erschien nur die auf dem Symposium vorgetragene Arbeit von Henning Stieve über die Orientierung der Meerestiere bei ihren Wanderungen.

Leider zeigte sich im Jahr 1960 auch der angegriffene Gesundheitszustand von Prof. Bückmann. Er trat vom Vorsitz der Deutschen Wissenschaftlichen Kommission für Meeresforschung zurück und gab die Leitung der BAH am 31.8. 1960 auf. Bis zur Ernennung eines Nachfolgers wurde Dr. Aurich mit der Wahrnehmung der Geschäfte des Direktors der BAH beauftragt. Nach der Übergabe der Dienstgeschäfte schrieb Prof. Bückmann an den zuständigen Ministerialdirigenten Dr. Meseck. „in diesem Augenblick empfinde ich das Bedürfnis, Ihnen noch einmal zu danken für Ihren Einsatz für die Interessen der Biologischen Anstalt Helgoland. ... Es war mir ein Vergnügen, daß Dr. Gunkel aus den USA schrieb, die Arbeitsmöglichkeiten an der Anstalt seien denen an den meisten dortigen Instituten überlegen. ... Ich empfehle nun die Biologischen Anstalt als unser gemeinsames Kind Ihrer weiteren Fürsorge, da ich ja leider nicht mehr die gesundheitliche Widerstandsfähigkeit habe, um für sie zu kämpfen. Dabei darf ich sicher noch einmal auf die Sorgen hinweisen, die mich in letzter Zeit bewegten. ... Nach ihrem ganzen Charakter und der Vielfalt ihrer Aufgaben gehört die Biologischen Anstalt Helgoland nicht in die Bundesforschungsanstalt für Fischerei. .. Für die Neubesetzung der Direktorstelle ist die Lösung von der BFA wesentlich; des Prestiges und der Möglichkeit einer angemessenen Dotierung wegen. Dagegen können und sollten alle Garantien gegeben werden für eine denkbar enge wissenschaftliche Zusammenarbeit mit den Instituten der Bundesforschungsanstalt für Fischerei...."

Prof. Bückmann war keine Zeit der Erholung vergönnt. Er hatte genügend Aufgaben an der Universität und blieb Direktor des Instituts für Fischereibiologie (IHF). Dieses war seit 1947 provisorisch im Dachgeschoß des Museums für Hamburgische Geschichte untergebracht, und im Jahr 1953 hatte Prof. Bückmann das IHF von Prof. Alfred Willer übernommen. Im Jahr 1964 konnte der seit 1956 geplante Neubau des IHF am Olbersweg in unmittelbarer Nachbarschaft der Bundesforschungsanstalt für Fischerei bezogen werden. Seit der Zeit der direktorialen Personalunion unter Prof. Bückmann werden von der BAH und dem IHF gemeinsame Kolloquien veranstaltet und auf diese Weise Gedankenaustausch und Zusammenarbeit zwischen den Mitarbeitern der beiden Institute gefördert. Prof. Max Gillbricht von der BAH war Dozent am IHF, und in jüngerer Zeit gilt dieses für Prof. Friedrich Buchholz (Helgoland) sowie für Prof. Karsten Reise (List). 


\section{Koordination der Meeresforschung im Nachkriegs-Deutschland}

Die traditionsreiche Arbeit der Deutschen Wissenschaftlichen Kommission für Meeresforschung betraf vor allem Untersuchungen über die Fischbestände, ihre Nutzung und über biologische sowie hydrographische Grundlagen. Zusätzlich wurden das Bundesministerium für Ernährung, Landwirtschaft und Forsten beraten, der Einsatz der Fischereiforschungsschiffe geplant und die Mitarbeit verschiedener Institute angeregt.

Ein Ersuchen des Haushaltsausschusses des Bundestages an die Bundesregierung führte dazu, daß der Bundesernährungsminister Ende 1955 die sogenannte MahlowKommission einsetzte. Ständige Mitglieder waren Ministerialrat a.D. Mahlow, Dr. Wilbrandt und Prof. Lembke. Die Mahlow-Kommission sollte zum einen alle vom Ministerium finanzierten Institutionen dahingehend prüfen, ob und wie sie die ihnen übertragenen Forschungsaufgaben erfüllten, zum anderen Empfehlungen für die zukünftige Arbeit geben. Nach etwas mehr als einjähriger Untersuchung lag ein über 80 Seiten langer Bericht vor (Anonymus, 1957), in dem auch die Institute der Bundesforschungsanstalt für Fischerei begutachtet wurden. Eine Übersicht und Abstimmung der Meeresforschung in der Bundesrepublik konnte dabei allerdings nicht erreicht werden, da die Länder wesentliche Teile an den Universitäten und in den Instituten der Max-Planck-Gesellschaft finanzierten, die nicht Gegenstand des Mahlow-Gutachtens waren.

Die BAH wurde im Mahlow-Gutachten als ein der Bundesforschungsanstalt für Fischerei angegliedertes Institut behandelt. Durch diese Zuordnung, so hieß es, wäre es möglich, die BAH von Forschungen mit rein praktischer Zielsetzung weitgehend zu entlasten. Die BAH wollte sich jetzt "ausschließlich der Grundlagenforschung an wissenschaftlich bedeutsamen Objekten mit zeitgemäßer Fragestellung zuwenden". Es wäre wünschenswert, wenn der geplante Beirat bald ernannt würde und seine Tätigkeit aufnehmen könnte. Die zur Zeit bestehende "Kopfstation" sollte auch künftig beibehalten werden. Durch sie könnten die Verbindungen zu anderen Forschungsinstituten, zur Universität und den Bibliotheken gepflegt werden. An ihr würden die Fachgebiete Tierphysiologie und Mikrobiologie aufgebaut. Die Stellungnahme der Kommission war positiv. Die Errichtung der in Hamburg und Helgoland geplanten Neubauten sollte so schnell wie möglich erfolgen. Das Bestreben der BAH, Dienstunterkünfte und Studentenheim vom Institutsgebäude auf Helgoland zu trennen, sollte eingehend geprüft werden, da "vieles für die Richtigkeit dieses Vorschlages spricht". Allgemein wurde in dem Kommissionsbericht dargelegt, daB volkswirtschaftlich wertvolle und vordringliche Forschungsaufgaben nicht mehr aus Geldmangel zurückgestellt werden sollten, da die Wirtschaftskraft der Bundesrepublik sich günstig entwickelt hätte. Es wären Ausweitung und bessere Ausrüstung der bestehenden Arbeitsgruppen notwendig, nicht aber neue Institute. Eine knappe Mittelbemessung wäre eine unproduktive Verwendung von Steuergeldern. Institutsleiter im Bundesdienst würden gegenüber Hochschulprofessoren relativ niedrig bezahlt, und auch eine Anhebung der Bezüge der anderen Wissenschaftler und Techniker wäre nötig, um leistungsfähige Mitarbeiter zu halten. Wichtig und dringlich wären flexiblere Haushaltsansätze, aber auch Koordination und Dokumentation.

Im Sinne des Zusammenwirkens von Bund und Ländern bei der Förderung von Wissenschaft und Forschung entstand schließlich das Verwaltungsabkommen über die Errichtung eines Wissenschaftsrates. Bundeskanzler Dr. Adenauer sagte anläßlich der Unterzeichnung am 5.9.1957, daß damit zum ersten Mal auf deutschem Raum eine Ein- 
richtung geschaffen würde, "die einen Gesamtüberblick über die wissenschaftliche Arbeit in der Bundesrepublik geben und den Regierungen von Bund und Ländern Vorschläge für die Förderung der Wissenschaft unterbreiten soll“. Am 6. 2. 1958 wurde der Wissenschaftsrat mit einer Ansprache des Bundespräsidenten Prof. Heuss im Festsaal der Bonner Universität feierlich konstituiert. Die ersten Stellungnahmen des Wissenschaftsrates ab 1960 bezüglich der Meereskunde betrafen die Hochschulen mit der Empfehlung, die Meeresforschung an den Universitäten Hamburg und Kiel zu pflegen und enthielten Vorschläge zum Ausbau der einzelnen Fachgebiete.

Eine kräftige Förderung erhielt die deutsche Meereskunde durch die Deutsche Forschungsgemeinschaft (DFG; Böhnecke \& Meyl, 1962). Die Anregung dazu erfolgte über den Internationalen Rat der wissenschaftlichen Unionen (ICSU), in dem die DFG die Bundesrepublik vertrat, mit der Aufforderung, einen deutschen Ausschuß für das Scientific Committee on Oceanic Research (SCOR) zu gründen und sich künftig an internationalen Expeditionen zu beteiligen. So bemühte sich die DFG, die dafür notwendige personelle und materielle Basis zu schaffen. Im Jahr 1960 wurde dazu ein Schwerpunktprogramm beschlossen, das die Aufgabe hatte, die Planung für ein den Grundlagenwissenschaften der Ozeanographie dienendes Forschungsschiff voranzutreiben: 1964 wurde die "Meteor" fertiggestellt.

\section{Professor Kinne und die BAH im Jahr 1962}

Nachfolger von Prof. Bückmann als Direktor der BAH wurde am 1.5.1962 Prof. Otto Kinne (Abb. 14) und damit ein junger Zoologe, der erst nach dem zweiten Weltkrieg in Tübingen und Kiel studiert hatte. Nach der Promotion 1952 in Kiel bei Prof. Adolf Remane und Prof. Hermann Friedrich über "Experimentelle Untersuchungen zur Biologie, Ökologie und Physiologie von Gammarus duebeni "arbeitete er weiter mit Crustaceen, Hydroidpolypen und Fischen auf dem Gebiet der physiologischen Ökologie am Zoologischen Institut in Kiel sowie als Forschungszoologe im Zoology Department der University of California in Los Angeles (Peters, 1984). Im Jahr 1958 ging er als Professor an die Universität Toronto in Kanada und wurde mehrfach vom Marine Biological Laboratory in Woods Hole als Instructor des Marine Ecology Course eingeladen. In Kiel habilitierte er sich für das Fach Zoologie mit der Arbeit "Über den Einfluß des Salzgehaltes und der Temperatur auf Wachstum, Form und Vermehrung bei dem Hydroidpolypen Cordylophora caspida (Palas), Thecata, Clavidae". Auch seine folgenden Arbeiten bemühten sich um ein Verständnis der Ökologie der Meeresbewohner, so wie es an der BAH eine gute Tradition ist.

Prof. Kinne konnte sein neues Institut gleich bei einem festlichen Akt vertreten. Am 1. 6. 1962 wurden durch den Bundesernährungsminister Schwarz die neuen Gebäude der Bundesforschungsanstalt für Fischerei in Hamburg-Altona feierlich eingeweiht (MeyerWaarden, 1962). Die BAH wurde dabei als "das älteste deutsche Institut für Meeres- und Fischereiforschung “ gewürdigt. Die Kopfstation der BAH bekam im Vergleich zu den anderen Instituten den weitaus größten Teil der Nutzfläche im "Haus Herwig“ an der Palmaille, und zwar die Labors und Büroräume des dritten und vierten Stockwerks, weiterhin Platz für die Bibliothek in der zweiten Etage sowie Kellerräume für Aquarien und Werkstätten (Abb. 15). Sehr günstig für die BAH war auch die Möglichkeit zur Nutzung des Hörsaals und der Gästezimmer im fünften Stock für Dienstaufenthalte der Mitarbei- 


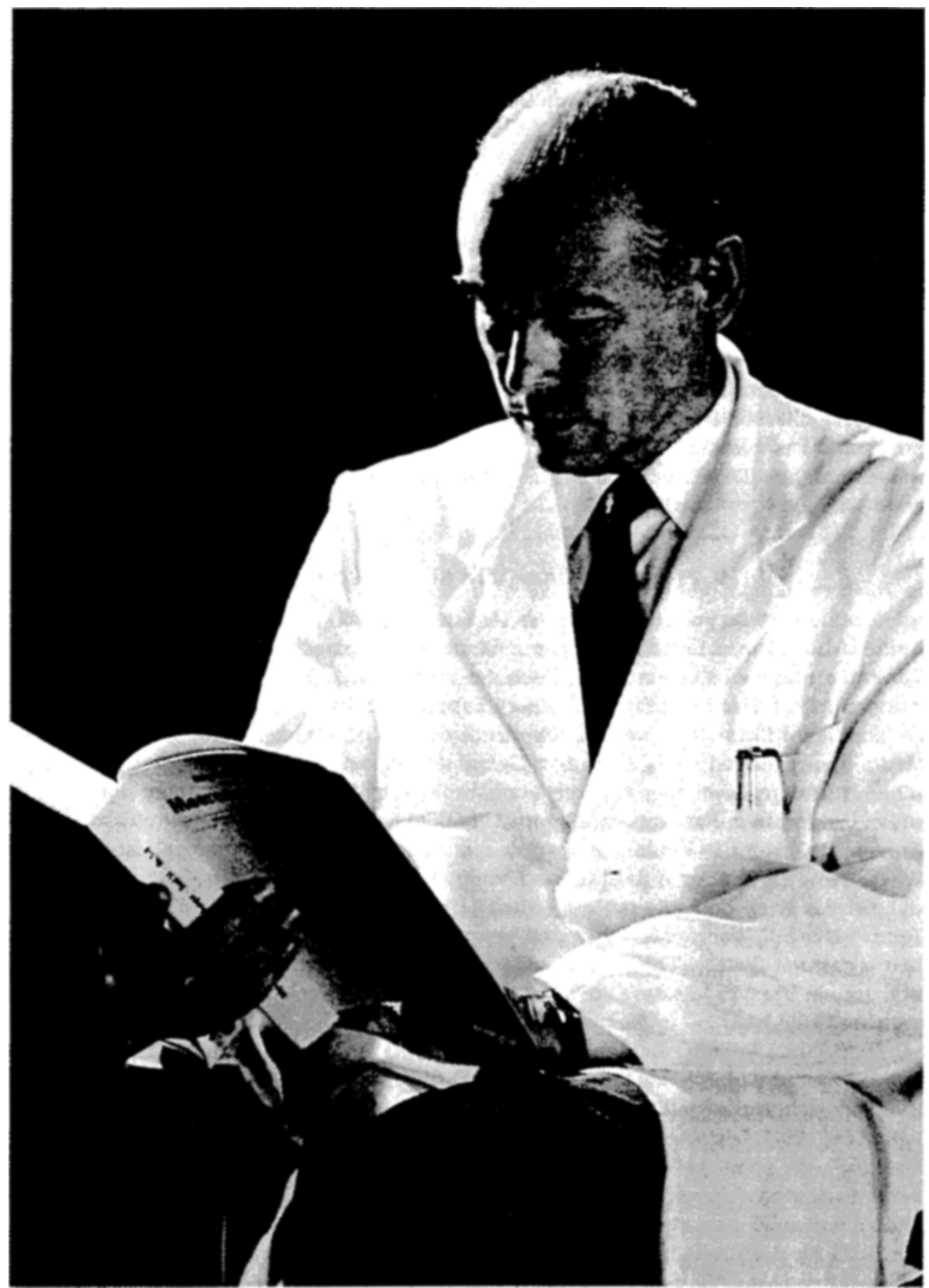

Abb. 14. Prof. Otto Kinne am 16. 8. 1967. Photo: Gerrit Sahling. 


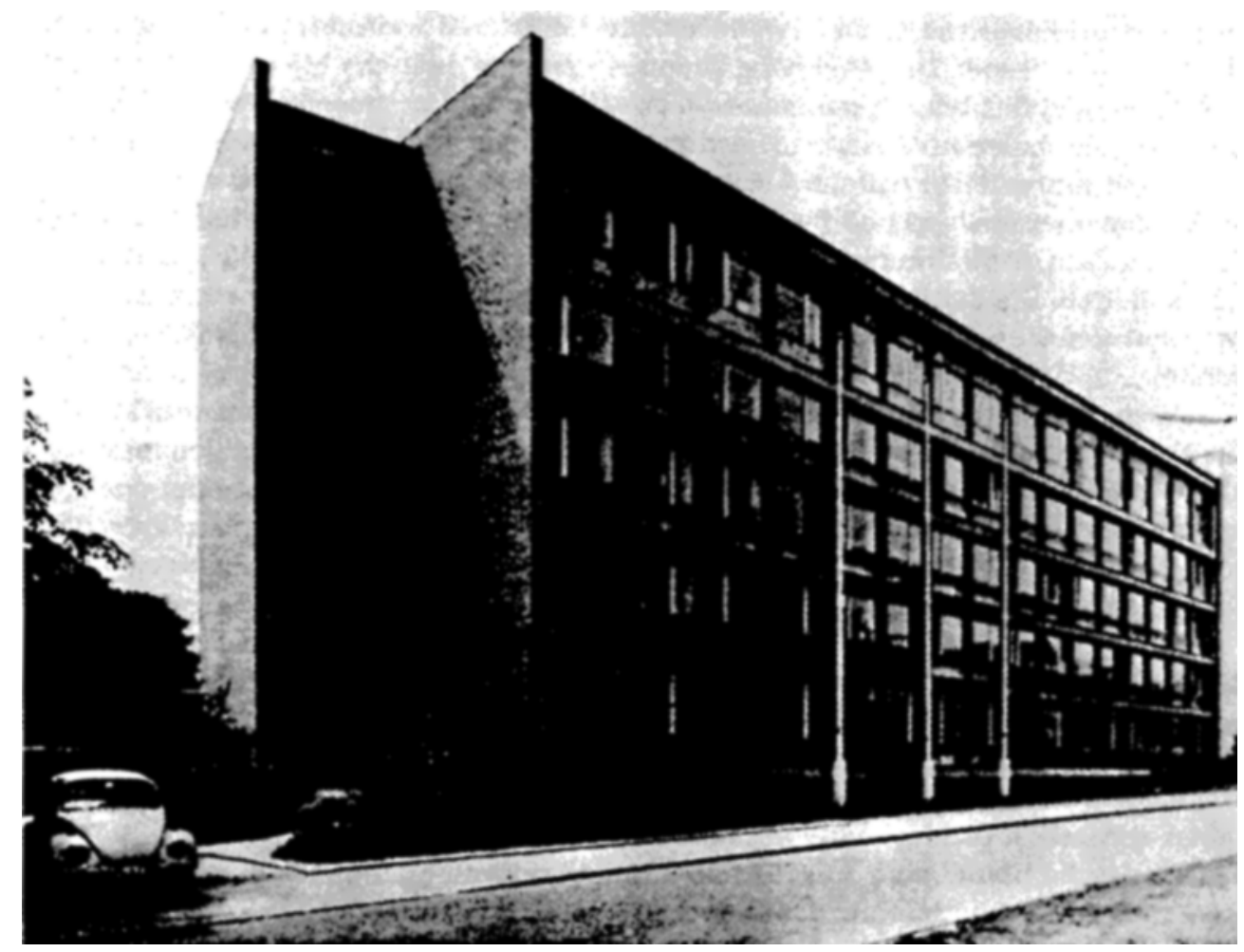

Abb. 15. Haus Herwig der Bundesforschungsanstalt für Fischerei an der Palmaille in HamburgAltona. Räume der BAH im 2., 3. und 4. Stock. Photo: Archiv der BAH.

ter der Inselstationen. Für den Umgang mit radioaktiven Stoffen und für strahlenbiologische Untersuchungen war ein besonderes Laborgebäude vorgesehen. Dieses wurde vom Deutschen Hydrographischen Institut gemeinsam mit der Bundesforschungsanstalt für Fischerei geplant und Ende 1969 in Hamburg-Sülldorf fertiggestellt.

Bald nach dem Amtsantritt von Prof. Kinne, am 2.6.1962, fand die konstituierende Sitzung des Kuratoriums der Bundesforschungsanstalt für Fischerei in Hamburg statt. Der Beirat der BAH sollte aufgelöst werden, und geplant war ein neuer Gesamtbeirat der Bundesforschungsanstalt für Fischerei und der BAH als 5. Institut (hier und im Folgenden nach Prof. Kinne, mdl. Mitteilung). An der Gründungsversammlung nahmen teil: Vertreter des BML (Abteilungsleiter I, MinDir Dr. Brettschneider, MinDirig Dr. Meseck und andere), die Mitglieder des neuzugründenden Beirats unter Vorsitz von Prof. Günter Dietrich (Kiel), die Direktoren der vier Fischereiforschungsinstitute der Bundesforschungsanstalt für Fischerei und Prof. Kinne. Außer der Gründung des neuen Beirats waren die Zusammenlegung der Verwaltungen, Bibliotheken und Schiffe vorgesehen. Prof. Kinne legte dar, daß bei seinen Berufungsverhandlungen von derartigen Vorhaben keinerlei Rede gewesen war und daß die Umsetzung seines Forschungsprogramms, das zu seiner Emennung zum Direktor geführt hatte, bei einer derartig engen Verklammerung von Fischereiforschung und Meeresbiologie nicht möglich sei. Fischereiforschung 
und meeresbiologische Grundlagenforschung hätten weltweit verschiedene fachliche Kontakte und Träger. Die BAH habe zudem als einzige deutsche Meeresstation traditionsreiche Verpflichtungen gegenüber Universitäten und anderen festländischen Forschungs- und Lehreinrichtungen (Gastforschung, Kurse, Materialversand). Diese Argumentation führte dazu, daß der Gesamtbeirat nicht berufen wurde. Die Auseinandersetzungen über Stellung und Einordnung der BAH begannen nun auch für Prof. Kinne, eben mit dem Zentralproblem, daß es damals keinen anderen Träger für die BAH als das BML gab. Die der BAH gestellten Aufgaben stimmten aber nicht in ausreichendem Maß mit den Zuständigkeiten dieses Trägers überein (Ende der mdl. Mitt. von Prof. Kinne).

Auch die am 14.6.1962 tagende Deutsche Zoologische Gesellschaft drückte ihre Sorge darüber aus, daß die BAH ihre Selbständigkeit verloren habe und von Instanzen abhängig sei, die der Grundlagenforschung fernstünden. Seit der Zuordnung der BAH zur "Verwaltung für Enährung, Landwirtschaft und Forsten des Vereinigten Wirtschaftgebietes“ ab 1.4.1948 paßten die traditionellen Aufgaben der BAH nicht zu den Ressortkompetenzen der Träger.

Prof. Kinne mußte also gleich nach seinem Amtsantritt um die Eigenständigkeit seines Instituts kämpfen. Sein Ansprechpartner im Bundesministerium für Ernährung, Landwirtschaft und Forsten (BML), Ministerialdirigent Dr. Meseck, hatte sehr viel für die Fischereiforschung getan und auch die BAH gefördert, wollte diese aber nicht aus dem Einflußbereich seines Ministeriums entlassen. Das lange und mühselige Tauziehen führte schließlich dazu, daß die BAH am 1. 1. 1971 durch Erlaß des damaligen Bundeskanzlers Willy Brandt vom 7.7.1970 dem Bundesministerium für Bildung und Wissenschaft unterstellt wurde. Kurz danach erhielt Prof. Kinne aus dem Hause des neuen Trägers einen Anruf. "Herr Kinne, haben Sie das Erdbeben in Hamburg gespürt?" "Nein" , sagte Prof. Kinne, "welches Erdbeben?" "Herr Meseck hat hier in Bonn so getobt, daß Sie das eigentlich in Hamburg hätten spüren müssen!“ Auch unter dem neuen Ministerium gab es jedoch Differenzen zwischen Trägerkompetenzen und Aufgaben der BAF, bis in die jüngste Gegenwart hinein.

Auf Helgoland konnte für die BAH im Jahr 1962 das Hafenlabor fertiggestellt werden, mit für die Ichthyologie wichtigen Becken. Hier züchtete Dr. Jürgen Flüchter, der vor seinem Einzug ins Hafenlabor als Bordbiologe auf der "Anton Dohrn" fuhr, Seezungen.

Von 1962 an wurden die Jahresberichte der BAH gedruckt und veröffentlicht. Für das Jahr 1962 wurden darin 7 Abteilungen und 13 Wissenschaftler aufgeführt. (1) Zoologie: Prof. Otto Kinne (Leiter, Hamburg), Dr. Bernhard Werner (Hamburg), Dr. Erich Ziegelmeier (List), Dr. Gotram Uhlig (Helgoland); (2) Physiologie: Prof. Friedrich Krüger (Leiter, Hamburg), Dr. Friedrich-Wilhelm Tesch (Hamburg); (3) Botanik: Dr. Peter Kornmann (Leiter, Helgoland), Dr. Hanswerner Kesseler (Helgoland); (4) Mikrobiologie: Dr. Wilfried Gunkel (Leiter, Helgoland); (5) Planktonforschung: Dr. Horst Aurich (Leiter, List), Dr. Max Gillbricht (Helgoland); (6) Fischbiologie: Dr. Adolf Kotthaus (Leiter, Hamburg), Dr. Jürgen Flüchter (Helgoland); (7) Strahlenbiologie: Dr. H. Aurich (Leiter, List), Dr. Klaus-Jürgen Götting (Helgoland). Dazu kamen 44 Mitarbeiter in Labors, Verwaltung, Bibliothek, Schiffsbetrieb, Aquarium und Versand, Werkstätten und Hausverwaltung, deren Namen ab 1964 auf den Heftumschlägen der "Helgoländer Wissenschaftlichen Meeresuntersuchungen“ (HWM) genannt wurden. 
Die laufenden Arbeiten der BAH wurden im Jahresbericht 1962 wie folgt beschrieben.

(1) Zoologie: Untersuchungen über den Einfluß von Temperatur und Salzgehalt auf Struktur und meristische Merkmale von eurysalinen Knochenfischen (Prof. Kinne); Studium von Ökologie, Biologie und Entwicklung von Hydrozoen mit Hilfe der Zuchtmethode (Dr. Werner); Auswertung von Bodenfaunaproben aus der Deutschen Bucht (Dr. Ziegelmeier); Kultivierung verschiedener Protozoen und Studien ihrer Entwicklungszyklen, Morphogenese und Cytologie (Dr. Uhlig).

(2) Physiologie: Untersuchungen zur physiologischen Ökologie von Arenicola; theoretisch-mathematische Bearbeitung von Wachstumskurven (Prof. Krüger).

(3) Botanik: Entwicklungsgeschichtlich-taxonomische Untersuchungen an Grünund Braunalgen (Dr. Kornmann); Untersuchungen über die Atmungsintensität mariner Algen (Dr. Kesseler).

(4) Mikrobiologie: Bakterienverbreitung und ihre Abhängigkeit von physikalischen und chemischen Umweltfaktoren an verschiedenen Stationen im Gebiet um Helgoland; Temperaturresistenz und Salzgehaltseinfluß bei marinen und limnischen Bakterienpopulationen (Dr. Gunkel).

(5) Planktonforschung: Dynamik und Ausbreitung des Zooplanktons im Gebiet der Polarfront (Dr. Aurich); methodische Vorarbeiten zur Analyse der gelösten organischen Verbindungen im Meerwasser; hydrographisch-planktologische Terminbeobachtungen um Helgoland (Dr. Gillbricht).

(6) Fischbiologie: Fischbestandsuntersuchungen in der Nordsee; Auswertung von Markierungs-Experimenten; Altersbestimmung und Züchtung von Seezungen (Dr. Kotthaus, Dr. Flüchter).

(7) Strahlenbiologie: Reinkultur einzelliger mariner Grünalgen (Dr. Götting).

Zur Förderung von Gedankenaustausch und Zusammenarbeit in der BAH begann Prof. Kinne mit den jährlich einmal in Hamburg stattfindenden Gesamtkonferenzen für die an den drei Standorten arbeitenden Wissenschaftler. Zu den Berichten und notwendigen Bekanntmachungen kamen Aussprachen über die Mittelverteilung in Haushalt und Bibliothek, meistens auch ein wissenschaftlicher Teil mit Vorträgen und Diskussionen sowie häufig ein gemütliches Zusammensein.

Die Organisation und die Personalaufstellung der BAH im Jahr 1964 sind aus Tabelle 1 ersichtlich.

\section{Das Schwerpunktprogramm ab 1963}

Im Jahr 1963 fand auf Einladung von Prof. Kinne das vierte Meeresbiologische Symposium in der Zentrale der BAH in Hamburg mit einer großen Zahl ausländischer Teilnehmer statt. Bei dieser Gelegenheit stellte Prof. Kinne sein neues Schwerpunktprogramm vor, nämlich Beobachtung und Analyse der "ökologischen Dynamik" in der Deutschen Bucht sowie die Untersuchung kausaler Wirkungszusammenhänge in Laborversuchen. Grundlage waren die Probenserien von Planktologen und Mikrobiologen auf Helgoland-Reede an der "Kabeltonne“. Die Kabeltonne liegt jetzt nicht mehr aus, sondern steht seit 1990 als Denkmal für die unermüdliche Arbeit in Vergangenheit und Gegenwart vor dem Helgoländer Aquarium (Abb. 16), die Probennahme an der Position $54^{\circ} 11,3^{\prime} \mathrm{N}, 7^{\circ} 54,0^{\prime} \mathrm{E}$ wird bis heute fortgesetzt. Dazu kamen die monatlichen Fahrten der "Uthörn" von Helgoland zur Elbe, gelegentlich auch zur Wesermündung, 


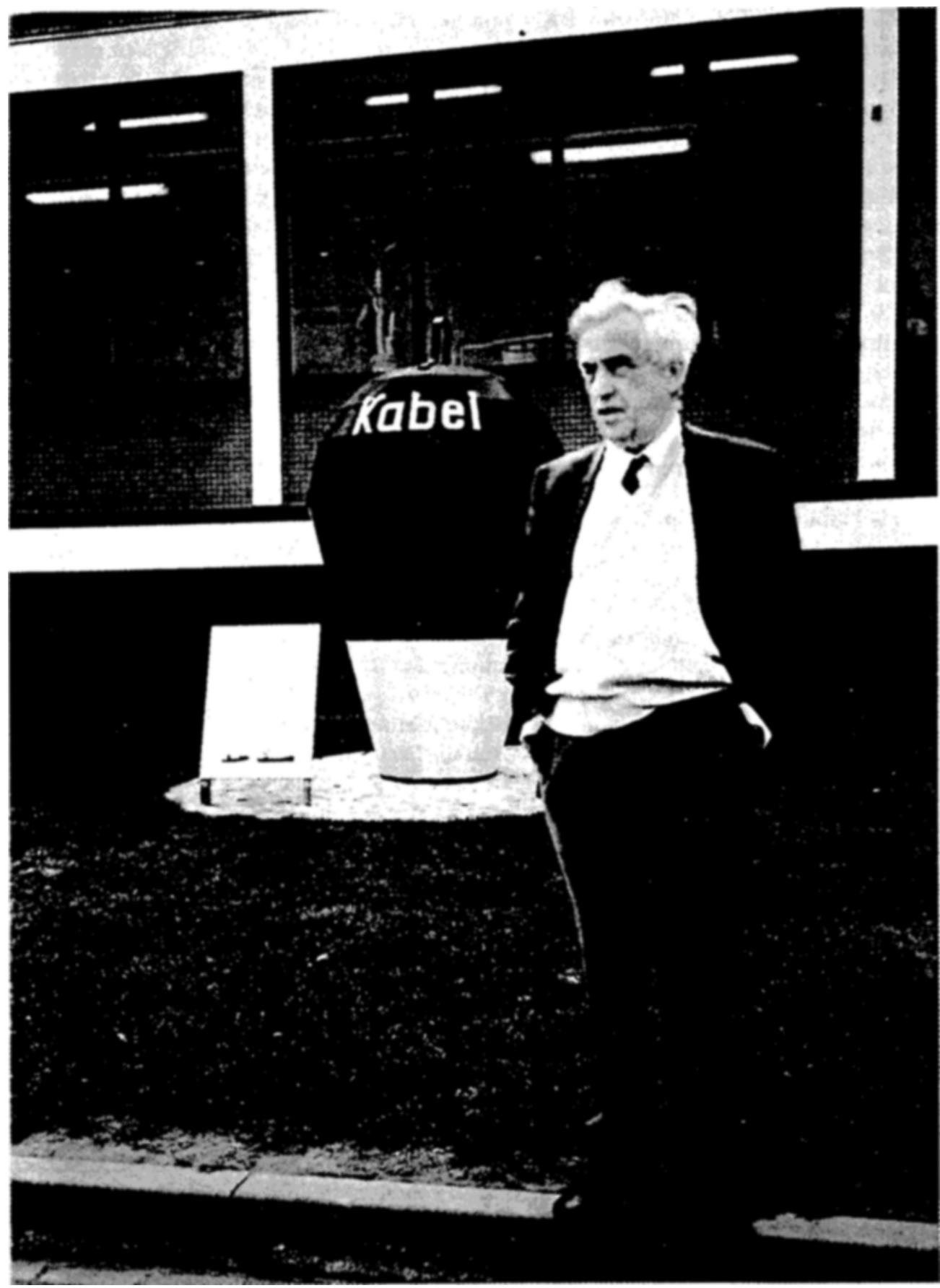

Abb. 16. Prof. Max Gillbricht, Mitinitiator der "Kabeltonnen-Proben”, mit der an Land vor dem Aquarium auf Helgoland zur Ruhe gegangenen Tonne (1991). Photo: Archiv der BAH. 
zur Hever und seewärts bis zum Feuerschiff "P8 " mit Probennahmen aus der Wassersäule und vom Meeresboden (Abb. 17).

Als Voraussetzungen zur Verwirklichung seines Schwerpunktprogramms nannte Prof. Kinne: (1) Die Indienststellung eines zweiten Forschungskutters (die "Friedrich Heincke" wurde 1968 fertig), (2) Errichtung eines experimentell-ökologischen Laboratoriums mit modernen Einrichtungen für Züchtung und Leistungsanalyse von Meeresorganismen (das neue Ökolabor auf Helgoland wurde 1976 der BAH übergeben) und (3) die Erweiterung des wissenschaftlichen und technischen Stabes, die in den folgenden Jahren erreicht wurde, aber nur zum geringen Teil dem ursprünglich konzipierten Schwerpunktprogramm zugute kam.

Veröffentlichungen aus dem Schwerpunktprogramm der BAH betrafen die Benthosproben und vor allem die "Helgoland-Reede-Serie", die auch im Hamburger Institut für Meereskunde bearbeitet wurde (Radach \& Berg, 1986, Hickel et al. 1993, Gillbricht 1994). Es wird gearbeitet an der ursprünglich beabsichtigten Darstellung der sehr komplexen und variablen "ökologischen Dynamik“ in der Deutschen Bucht. Die diesbezüglichen Bemühungen von Dr. Greve und die Zusammenarbeit mit den Physikern des Bun-

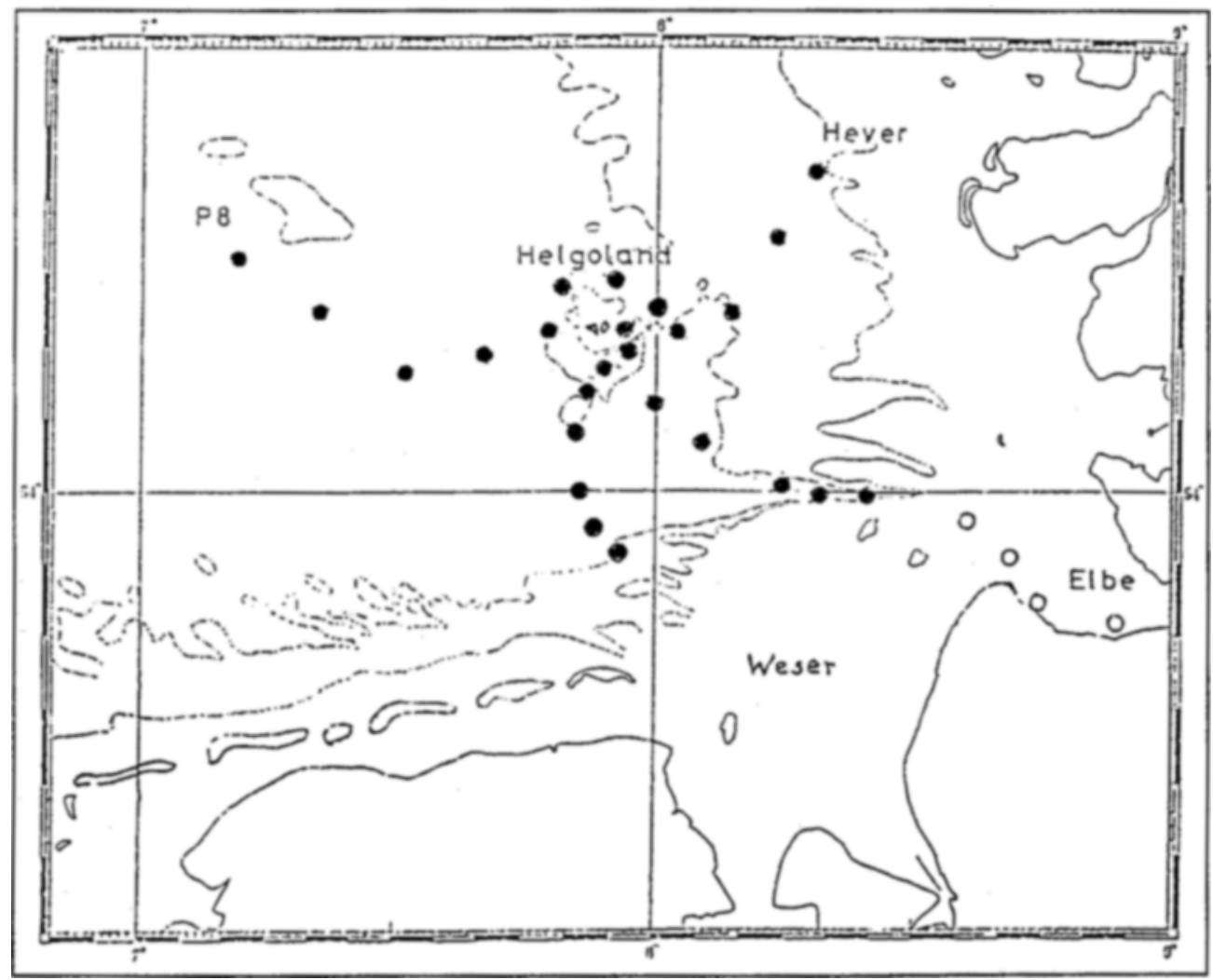

Abb. 17. Positionen der Probennahmen auf den Schnitten und der Inselrundfahrt des "Schwerpunktprogramms". 
desamtes für Seeschiffahrt und Hydrographie sowie die Modelle aus anderen Teilen der Nordsee (Fransz, Mommaerts \& Radach, 1991) lassen auf weitere Einsichten und Publikationen hoffen. Anfangs ahnte man nicht in vollem Umfang, daß die im Schwerpunktprogramm gesammelten Daten später eine große Bedeutung für die Erkennung langfristiger Veränderungen in der Deutschen Bucht bekommen würden.

\section{Symposien und Arbeitstreffen an der BAH bis 1983}

Die wiederbesiedelte Insel Helgoland mit dem neu erstandenen Institut erwies sich als günstiger Ort für wissenschaftliche Tagungen. Nach dem Ersten Deutschen Meeresbiologischen Symposium auf Helgoland 1960 gab es im September 1963, von Prof. Kinne, Prof. Krüger und Dr. Alfred Locker (Wien) organisiert, das "Erste internationale Symposium über Probleme der quantitativen Biologie des Stoffwechsels", das zugleich als 1. Internationales Helgoland Symposium (1. IHS) gezählt wurde (Übersicht der IHS bei Kinne, 1984, p. 2). Führende Wissenschaftler erörterten physiologische, biochemische, biophysikalische und mathematische Arbeiten, und 35 Referate wurden in den "Helgoländer wissenschaftlichen Meeresuntersuchungen" (HWM, Band 9, 1964) veröffentlicht. Noch im gleichen Jahr folgte das nach Helgoland (1960), Bremerhaven (1961) und Kiel (1962) "Vierte Meeresbiologische Symposium" mit dem Titel "Biology, Ecology and Physiology of Marine Organisms" in der Zentrale der BAH in Hamburg mit 130 Teilnehmern aus 14 Ländern (HWM, Band 10, 1964). Das Zweite Internationale Symposium über die quantitative Biologie des Stoffwechsels, zugleich als 3. Internationales Helgoland Symposium gezählt, fand 1965 wieder auf Helgoland statt, mit 57 Wissenschaftlern aus 16 Ländern. Die gedruckten Vorträge mit Diskussionen füllten Band 14 der HWM (1966).

Es war Prof. Kinnes Idee, die auf Helgoland 1960 begonnenen Treffen der deutschen Meereskundler auf einen europäischen Kreis von Meeresbiologen auszudehnen, mit Gästen aus anderen Erdteilen. Zum "Ersten Europäischen Symposium über Meeresbiologie" im Jahr 1966 lud Prof. Kinne nach Helgoland ein und stellte drei Themen in den Vordergrund: "Experimental Ecology - its Significance as a Marine Biological Tool “, "Subtidal Ecology - Particularly as Studied by Diving Techniques" und "The Food Web in the Sea". An diesem "First European Marine Biology Symposium (EMBS) " nahmen 236 Wissenschaftler aus 24 Ländern teil (Abb. 18), und es gab auch wesentliche Beiträge der BAH-Mitarbeiter. Die Meeresforschung, die durch die große Zahl von Institutionen und Forschern unübersichtlich und unpersönlich zu werden drohte, konnte wieder vom unmittelbaren Gedankenaustausch profitieren. Vorträge und Diskussionen dieses Symposiums, zugleich als 4. Internationales Helgoland Symposium gezählt, füllten mit 669 Seiten Band 15 der HWM. Während des 1. EMBS berief Prof. Kinne ein Komitee aus führenden Meeresbiologen und schuf damit eine koordinierende Instanz, die bis heute für die Kontinuität der EMBS sorgt. Das 2. EMBS fand 1967 auf Einladung von Prof. H. Brattström in Bergen (Norwegen) statt. Zum 3. EMBS im Jahr 1968 lud Prof. Robert Weill nach Arcachon (Frankreich) ein. Diese Symposien in jährlichem Abstand trugen maßgeblich dazu bei, die weltweiten Kontakte zwischen den Meeresbiologen zu verbessern und lieferten eine Basis für die Zusammenarbeit meeresbiologischer Forschungseinrichtungen in Europa. Das EMBS wurde in der Folge zu einer festen Institution, das 32. EMBS fand 1997 in Kristineberg (Schweden) statt. 


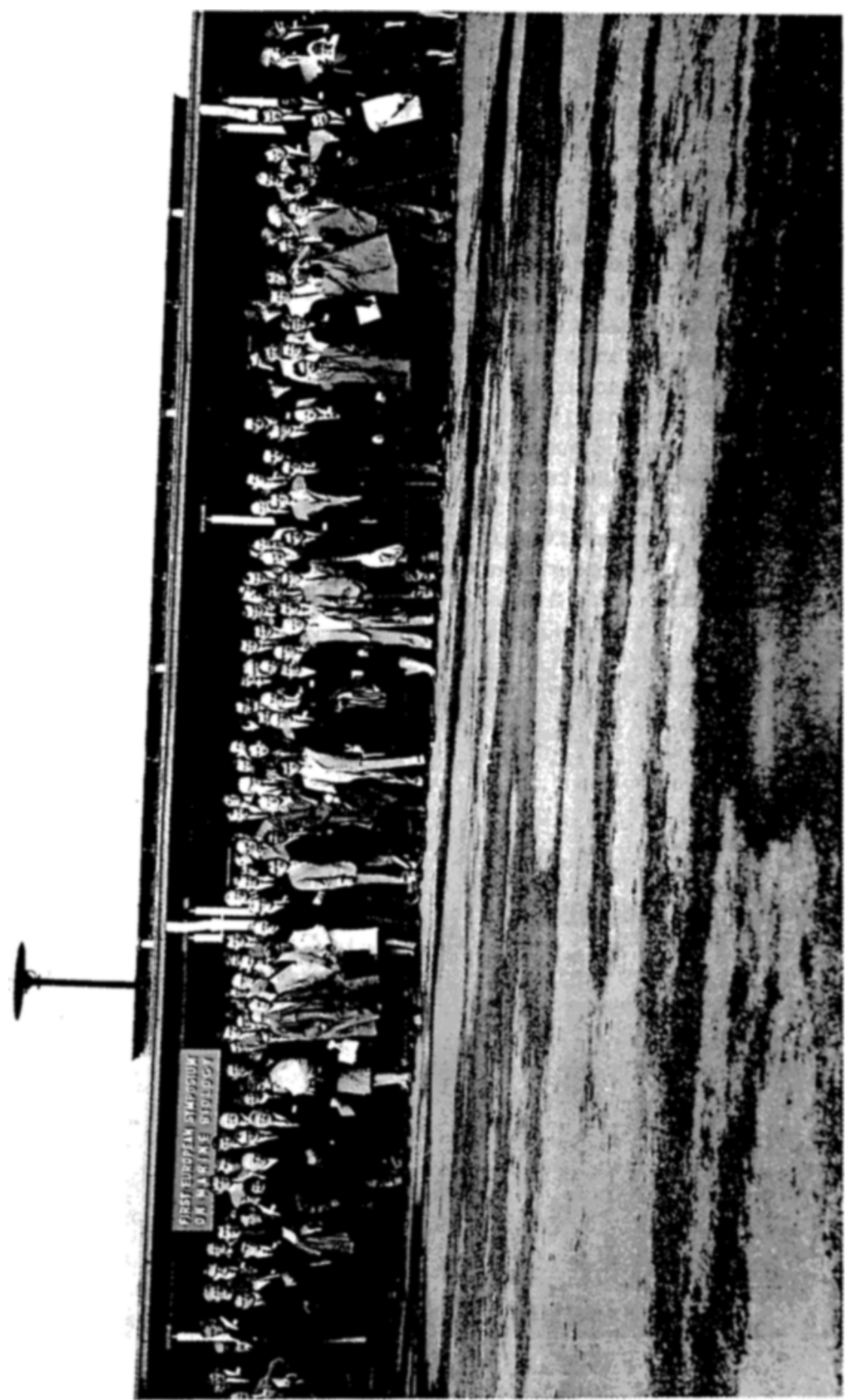

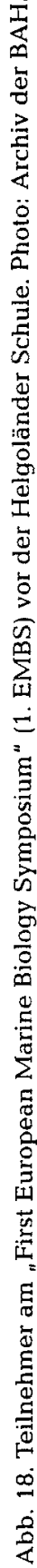


Im September 1967 feierte die BAH ihr 75jähriges Bestehen mit dem 5. Internationalen Helgoland Symposium. Es hatte ein Thema, das in den folgenden Jahren immer wichtiger werden sollte, "Biological and Hydrographical Problems of Water Pollution in the North Sea and Adjacent Waters". Auch aus der Arbeit der BAH wurden zahlreiche Ergebnisse vorgetragen. Die für 1969 angekündigte Verklappung saurer Industrieabwässer nordwestlich von Helgoland hatte eine Reihe von Untersuchungen über zu erwartende Auswirkungen auf die marine Flora und Fauna angeregt. Vorträge und Diskussionen wurden später in Band 17 der HWM gedruckt, mit abschließenden „Recommendations" zum Schutz der Nordsee und zur Intensivierung der Pollution-Forschung im marinen Bereich. Anschließend an das 5. IHS fand eine Arbeitssitzung über Gewässerverölung, Ölbekämpfung und Ölabbau statt.

Vorangegangen war im März 1967 ein durch die DFG im Rahmen ihres Schwerpunktprogramms "Litoralforschung" veranstaltetes Kolloquium "Abwassereinflüsse in Küstennähe" in Bremerhaven, an dem einige Wissenschaftler der BAH teilnahmen. Auch hier war die zunehmende Besorgnis über die Verschmutzung der See und die Notwendigkeit interdisziplinärer und zwischenstaatlicher Zusammenarbeit deutlich geworden.

Das 6. Internationale Helgoland Symposium wurde im September 1969 unter dem Motto "Cultivation of Marine Organisms and its Importance for Marine Biology “ veranstaltet. Es kamen 258 Wissenschaftler aus 30 Ländern in die Nordseehalle (Abb. 19), und es gab so viele Vortragsanmeldungen, daß 64 ausgesucht wurden, die zum großen Teil

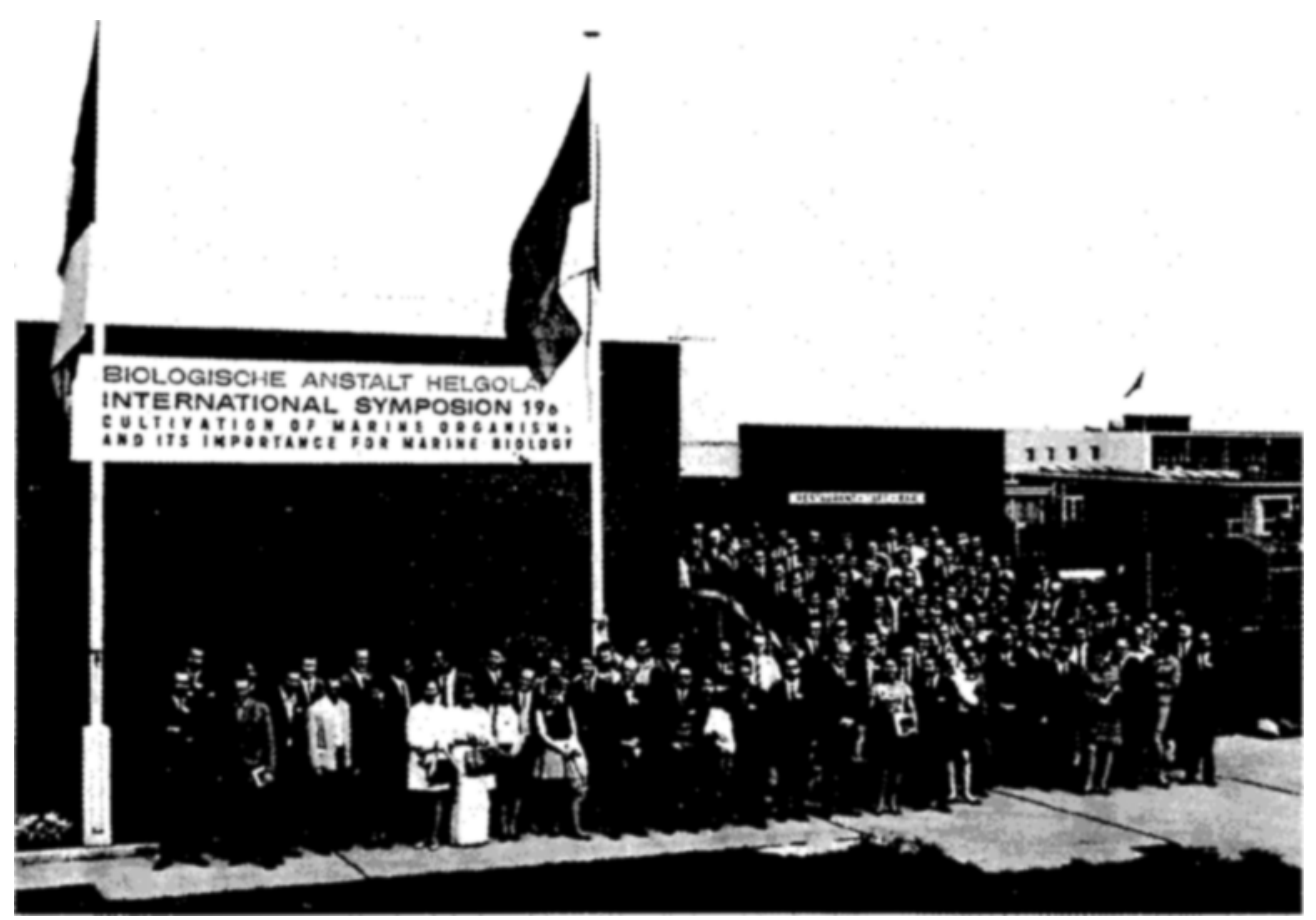

Abb. 19. Teilnehmer am Internationalen Helgoland Symposium 1969 vor der Nordseehalle. Photo: Höhler. 
in Parallelveranstaltungen stattfinden mußten (HWM, Band 20, 721 Seiten). Dazu wurden zwei fruchtbare "Informal Sessions" über "Cultivation of plankton organisms" und "Fish-farming, including farming of other organisms of economic importance" unter der Leitung von Dr. R. J. Conover und Dr. Gotthilf Hempel abgehalten (Heft 4 von Band 21 der HWM). Die Kultivierung von Meeresorganismen berührte einen Themenkreis, der an der BAH schon vor den beiden Weltkriegen bearbeitet wurde, etwa von Schreiber (1927), Hertling (1932), Hagmeier (1933) und auch von der jüngeren BAH-Generation, nämlich von Dr. Kornmann (Abb. 20), Dr. Drebes, Dr. Flüchter, Dr. Gunkel und Dr. Uhlig mit Beiträgen im Sammelwerk "Methoden der meeresbiologischen Forschung " (Schlieper, 1968). Die Züchtung mariner Organismen sollte in Zukunft durch neue Arbeitsmöglichkeiten im Experimentell-Ökologischen Labor besonders aktuell werden.

Im Jahr 1971 fand auf Einladung von Prof. Kinne die 65. Jahresversammlung der Zoologischen Gesellschaft (DZG) auf Helgoland statt (12.-18.9.). Die DZG hatte bei der Gründung der BAH Pate gestanden und die BAH beim Aufbau nach dem Zweiten Weltkrieg nachhaltig unterstützt (Geus \& Querner, 1990). Im Programmheft zu dieser Tagung stellte Prof. Kinne Gastforschung und Kursbetreuung an der BAH vor und teilte mit, daß die DFG zusätzliche Mittel für Personal, technische Neuanschaffungen, eine Handbibliothek und die Ausstattung von Speziallabors für Elektrophysiologie, Biochemie und Stoffwechselphysiologie, Photophysiologie sowie Histologie bewilligt hatte. Es wurde zu einem Empfang durch den Direktor der BAH im Kurhaus, zu organisierten Ausfahrten und Felswattexkursionen eingeladen. Dr. Werner schrieb zur Einstimmung der Helgoland-Besucher der DZG eine anschauliche Einführung mit Wissenswertem aus Geschichte und Topographie der Insel und sprach vom "geradezu magischen Zauber, den Helgoland auf die Menschen von nah und fern ausübt". Helgoland erwies sich immer wieder als gut geeigneter Ort, um Fachkollegen aus aller Welt in persönlichen Kontakt zu bringen und auch außerwissenschaftlich zu unterhalten, etwa mit Inselrundfahrten, Parties im Kurhaus, in der Netzhalle, am Dünenstrand oder im Schwimmbad.

Das 7. Internationale Helgoland Symposium (17.-22.9.1972) wurde unter das Thema "Man in the Sea - in situ Studies on Life in Oceans and Coastal waters" gestellt. In seiner Einführung gab Prof. Kinne eine geschichtliche Übersicht, angefangen bei Beebe und seiner Taucherkugel in den 30er Jahren sowie den Schwimmtauchern Cousteau, Gagnan und Hass in den 40er Jahren. Die Veröffentlichung der Beiträge in Band 24 der HWM repräsentierte eine Bilanz über Stand, Methoden, Probleme und Zukunft biologischer Unterwasserforschung von kalten bis zu tropischen Meeren.

Im Herbst 1976 fand das 8. Internationale Helgoland Symposium statt (26.9.-1.10.), bei dem das neue Experimentell-Ökologische Laboratorium vorgestellt werden konnte. Zum Thema "Ecosystem Research" wurden im Band 30 der HWM die Beiträge des Symposiums zusammengestellt. Unter "General aspects" findet sich ein plausibles, vereinfachtes Beispiel über trophische Beziehungen im Plankton zwischen Abwassereinfluß und der Entwicklung von Lachs oder Rippenquallen von Dr. Wulf Greve (BAH) und anschließend ein kritischer Artikel vom Altmeister Joel Hedgpeth unter dem Thema "Models and muddles". Nach Hedgpeth bringen uns vereinfachende Modelle nicht weiter, da sie die Natur nicht angemessen wiedergeben können. Dr. Malte Elbrächter (BAH) berichtete über Nährstoffkonkurrenz in Kulturen mit mehreren Planktonalgen, und unter dem Thema "Tidal ecosystems" schrieb Dr. Karsten Reise (BAH) über Versuche im Schlickwatt, bei denen Konsumenten ferngehalten wurden. Im Abschnitt "Benthic eco- 


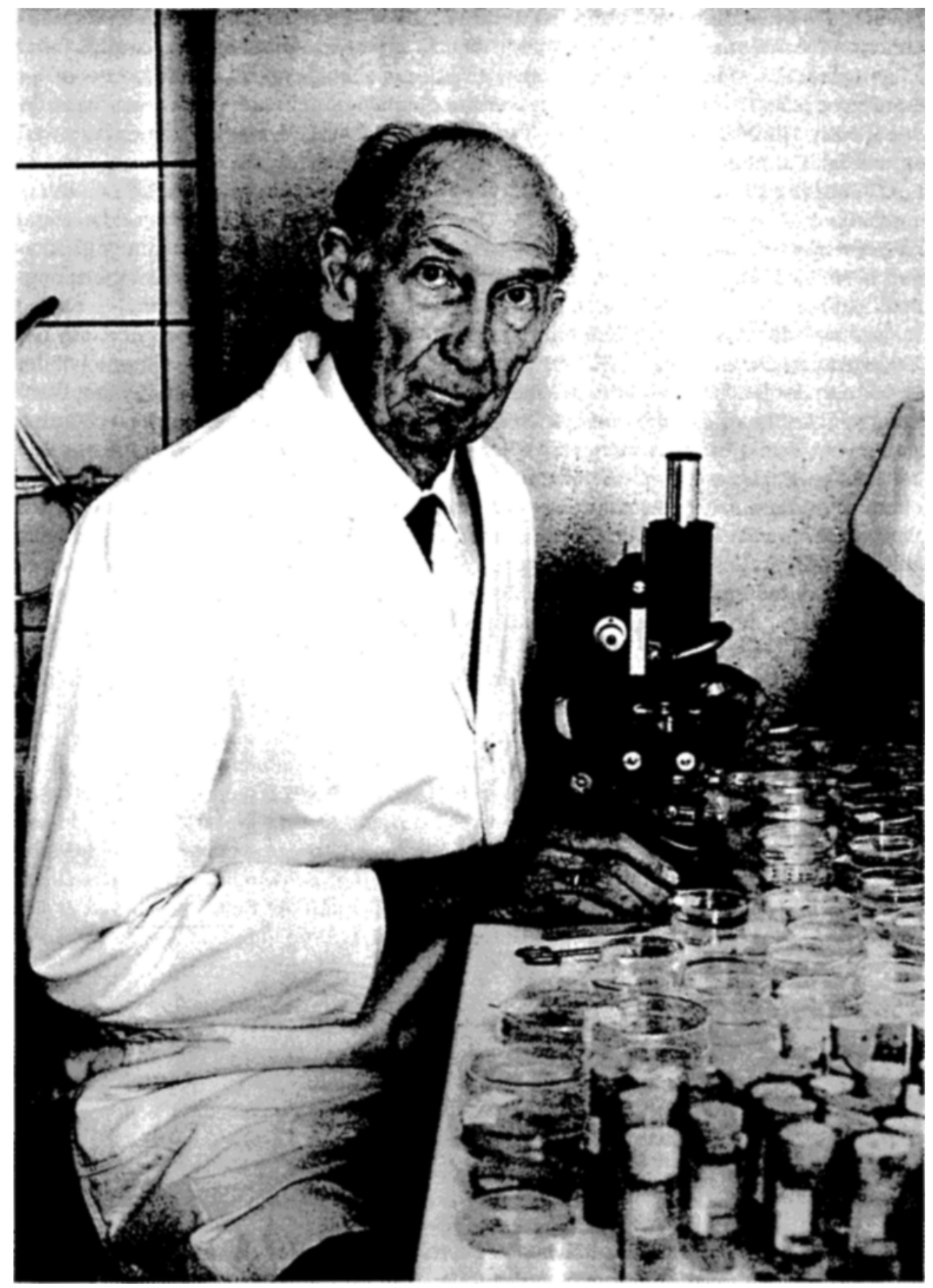

Abb. 20. Dr. Dr. h. c. Peter Kornmann, 1987. Photo: Gerrit Sahling. 
systems" berichtete Dr. B. Gulliksen (Trondheim) über das Makrobenthos in der Lübecker Bucht, das er bei zwei Aufenthalten im dort 1974 und 1975 stationierten Unterwasserlabor "UWL Helgoland" untersucht hatte. Unter dem Thema "Pollution effects on ecosystems" stand der Vortrag von Dr. Hermann Kayser (BAH) über die Wirkung von Zinksulfat auf die Vermehrung von einzelligen Algen in Mono- und Multispezies-Kulturen.

Im Jahr 1979 wurde wieder ein European Marine Biology Symposium, inzwischen das 14. EMBS, auf Helgoland veranstaltet (23.-29.9.), gleichzeitig als 9. Internationales Helgoland Symposium gezähit. Nach dem 1. EMBS auf Helgoland hatten Espegrend (Norwegen), Arcachon, Bangor (Wales), Venedig, Rovinj (Jugoslawien), Texel, Sorrento (bei Neapel), Oban (Schottland), Ostende, Galway (Irland), Stirling (Schottland) und Port Erin (Isle of Man) als weitere Tagungsorte des EMBS jährlich die Meeresbiologen zusammengeführt. "Schutz des Lebens im Meer" war das Thema 1979 auf Helgoland, und über 200 Wissenschaftler aus 25 Ländern kamen auf die Insel. Sie diskutierten über Wasserverunreinigungen sowie deren Wirkungen auf Meeresorganismen und Lebensgemeinschaften, weiter auch über die Möglichkeit, die Entwicklung von Gebieten, Arten und Ökosystemen zu "managen". Veröffentlicht wurden die Beiträge in Band 33 der "Helgoländer Meeresuntersuchungen " (HM), zuvor als "Helgoländer wissenschaftliche Meeresuntersuchungen" (HWM) bezeichnet, nun also im Zeitschriftentitel vereinfacht. Die Teilnehmer formulierten und verabschiedeten ein Memorandum, in dem sie ihre große Besorgnis ausdrückten „über die anhaltende Beeinträchtigung der marinen Umwelt und ihrer lebenden Naturschätze sowie über die Unzulänglichkeit der Gegenmaßnahmen ". Sie empfahlen wirksame, international vereinbarte Maßnahmen zum Schutz des Meeres.

Das 10. Internationale Helgoland Symposium im Jahr 1983 (11.-16.9.) war das letzte, zu dem Prof. Kinne einlud. Das Thema "Diseases of Marine Organisms" entsprach dem Titel des mehrbändigen Werkes, dessen Herausgabe er gerade organisierte (1980-1990) und das später vom Verlag Wiley \& Sons (Band 1), beziehungsweise der BAH (Bände 2-4), publiziert wurde. Band 37 der HM enthält 43 der vorgetragenen Arbeiten und den Bericht über die "Informal Session", die sich mit Fischkrankheiten befaßte. In seinem Schlußwort im Symposiumsband betonte Prof. Kinne, daß unser Wissen über Gesundheit und Krankheit im Ozean und in Küstengewässern noch sehr beschränkt ist, sich auf wenige Arten stützt und daß die Bedeutung von Krankheiten für ökologische Beziehungen und Theorien viel zu wenig berücksichtigt wird.

\section{Schiffe und Forschungsfahrten 1962-1983}

Im Jahr 1962 waren für die BAH folgende Schiffe von Helgoland aus in Fahrt:

FK "Uthörn” (Abb. 21), Kompositbau aus dem 2. Weltkrieg, $24 \mathrm{~m}$ lang, 6,40 m breit, 69 BRT; Kapitän Tönnies Hornsmann; Steuermann Jürgen Stoldt, Maschinist Peter Singer, biolog. Fischer und Netzmacher Nickels Wichers, Koch Günther Wolf.

MB "Ellenbogen" (Abb. 22), Stahlkonstruktion von 1949, 10,2 m lang, 3 m breit; Schiffsführer John Herzog, biolog. Fischer Werner Krühs.

Im Jahr 1966 wurde ein kleines, $6 \mathrm{~m}$ langes offenes früheres Helgoländer Fischerboot gekauft, das sich bei geringem Tiefgang von $0,45 \mathrm{~m}$ günstig für die Arbeiten im flachen Wasser im Felswatt einsetzen ließ. Mit dem Namen "Digger" (helgoländisch für 


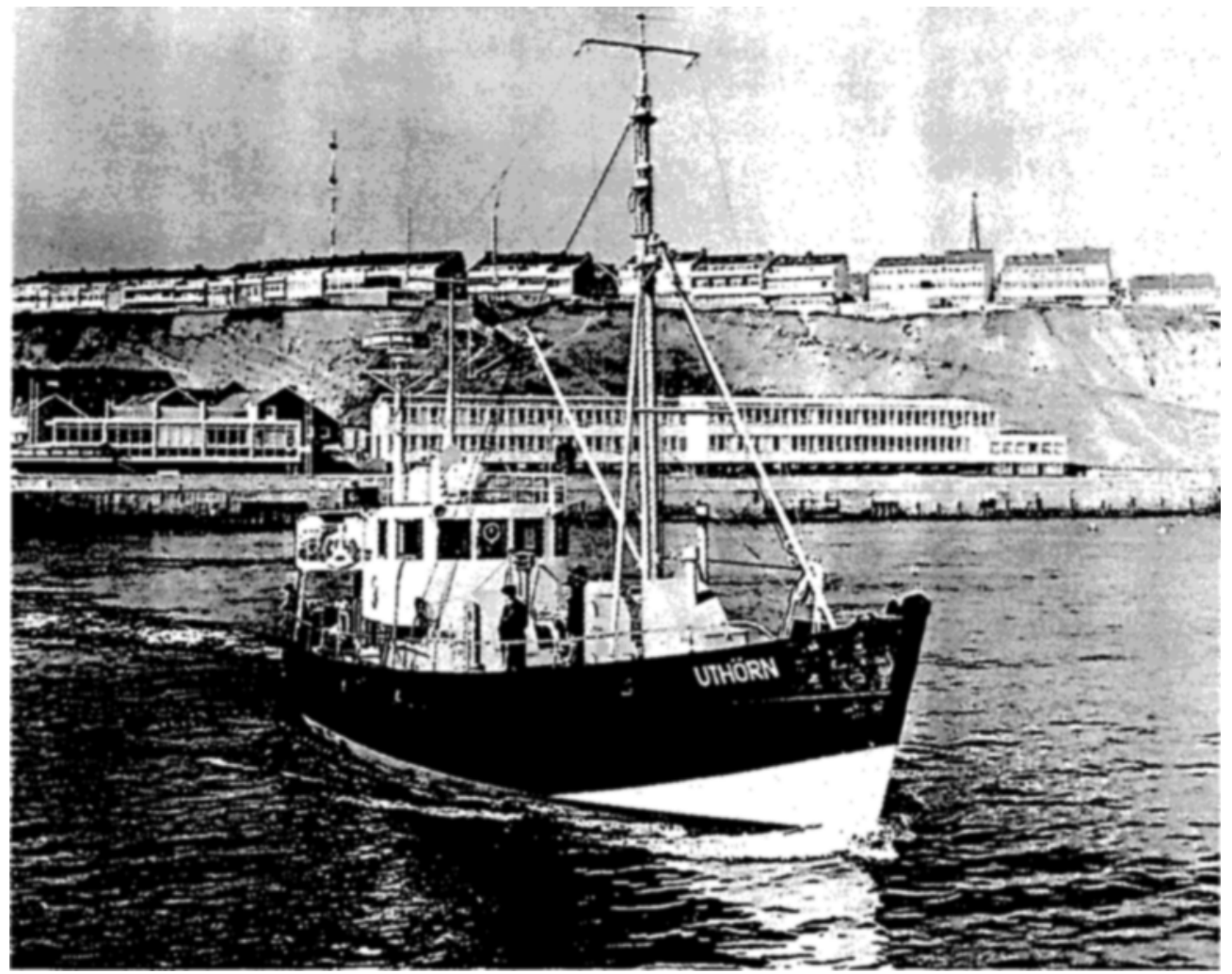

Abb. 21. FK "Uthörn", 1947-1982 im Dienste der BAH. Photo: Andreas Holtmann, 1971.

"Taucher") wurde die Zuordnung zur Tauchergruppe angezeigt. Erster Bootsführer war Werner Krühs.

In List wurde seit 1962 eingesetzt: MB "Gelk", Holzboot vom ehemaligen Seefliegerhorst, 8 m lang, 2,5 m breit. Bootsführer war Reimer Rickmers, später Adolf ("Addi “) Kruse.

Als eine der Voraussetzungen zur Verwirklichung des "Schwerpunktprogramms" zur Erforschung der Ökologie der Deutschen Bucht hatte Prof. Kinne einen zweiten Forschungskutter gefordert. Im Jahr 1966 konnte auf der Werft von August Pahl in Finkenwerder mit dem Bau begonnen werden. Auf dem Hauptdeck waren in günstiger Mittschiffslage 5 Arbeitsräume vorgesehen, ein Mehrzwecklabor, ein ichthyologisches Labor mit kardanisch aufgehängten Aquarien, ein chemisches, ein physikalisches und ein mikrobiologisches Labor mit Autoklavenraum. Am 31.10.1967 wurde das Schiff von Frau Maria Hagmeier, der Witwe des früheren Direktors Prof. Arthur Hagmeier, auf den Namen "Friedrich Heincke" getauft. Das Schiff war $38.2 \mathrm{~m}$ lang, $9 \mathrm{~m}$ breit und hatte einen Tiefgang von $3,5 \mathrm{~m}$. Ein 920 PS MWM-Diesel ermöglichte eine Geschwindigkeit von 12,5 Knoten. Am 10.6. 1968, einem sonnigen Tag, wurde die "Friedrich Heincke" (Abb. 23) von Hamburg nach Helgoland, zu ihrem Heimathafen, überführt und dort festlich emp- 


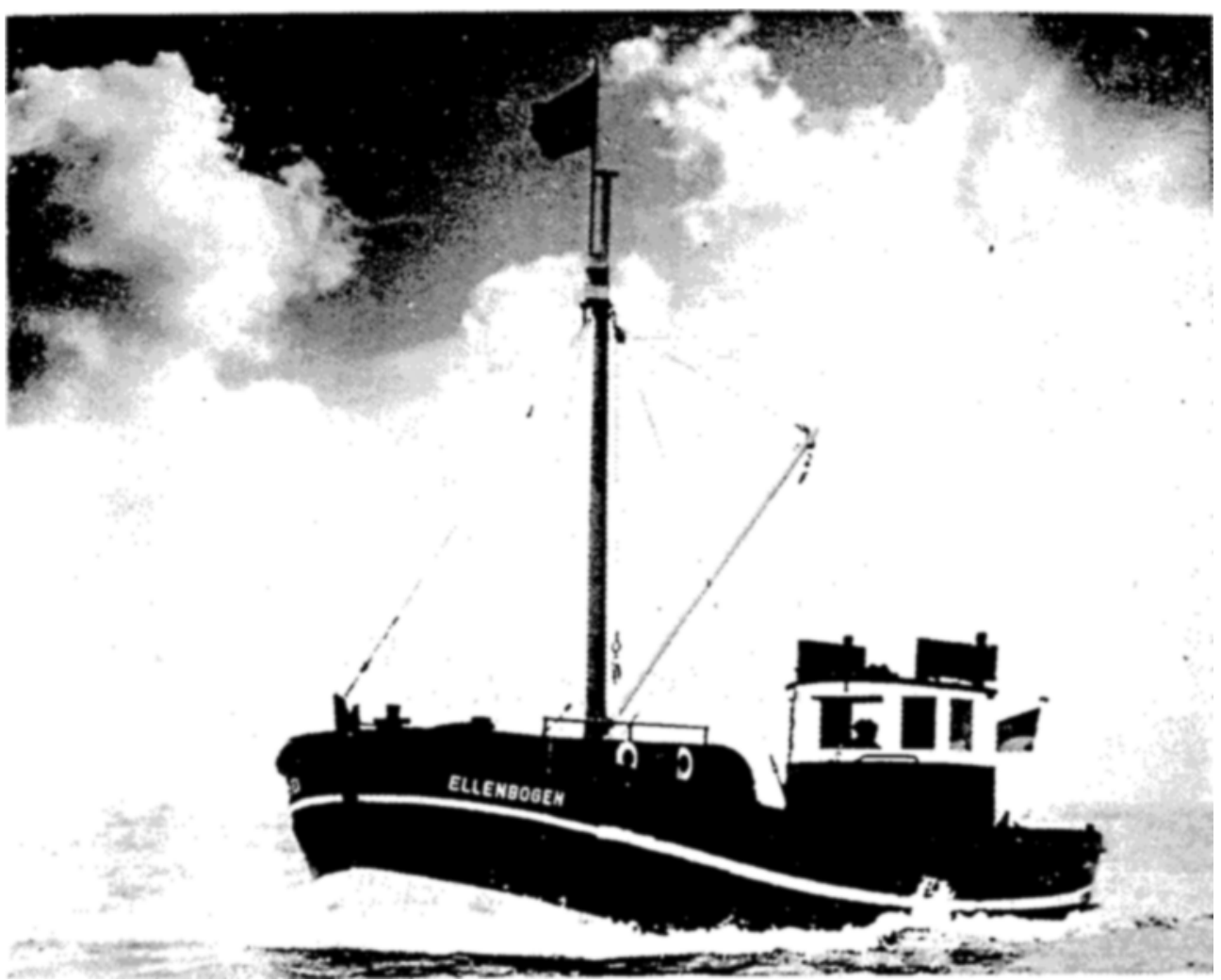

Abb. 22. MB “Ellenbogen”, 1949-1975 im Dienste der BAH. Photo: Jürgen Marschall, 1967.

fangen. Die Kinder bekamen an Bord ihre "Störkringel", die besonderen Brezeln für die Feier beim ersten Einlaufen eines neuen Schiffes im Heimathafen Helgoland. Die noch unvollständige Besatzung bei der Indienststellung bestand aus Kapitän Tönnies Hornsmann, Steuermann Hans Falke, Bootsmann und Netzmacher Nickels Wichers, 1. Maschinist Volker Oelrichs, 2. Maschinist Willi Lunter, Matrose Herbert Lanius und dem Koch Günther Wolf.

In den ersten Monaten des Jahres 1968 wurde ein umfangreiches Erprobungsprogramm durchgeführt, wobei die "Friedrich Heincke" sich als recht seetüchtig erwies. Bis zu Windstärke 8 konnte noch wissenschaftlich gearbeitet werden. Die erste Fernfahrt wurde von Prof. Kinne geleitet. Zum wissenschaftlich-technischen Stab gehörten Dr. Jürgen Flüchter, Dr. Wilfried Gunkel, Dr. Hanswerner Kesseler, Dr. Wolfgang Krumbein, Dr. Karlheinz Moebus, Dr. Friedrich-Wilhelm Tesch, Erich-Hartwig Harms und Hans-Hermann Trekel. Die Besatzung war verstärkt durch Karl-Heinz Hottendorf, Gerhard Jung und Günther Tadday. Untersuchungen im Ärmelkanal und in der Biskaya, ein Besuch beim 3. Europäischen Meeresbiologischen Symposium in Arcachon und Aaltransport sowie Aalverpflanzungen in Zusammenarbeit mit dem Rijksinstituut voor Visserijonderzoek in Ijmuiden wurden miteinander verbunden. Vom 19. September bis zum 4. Okto- 


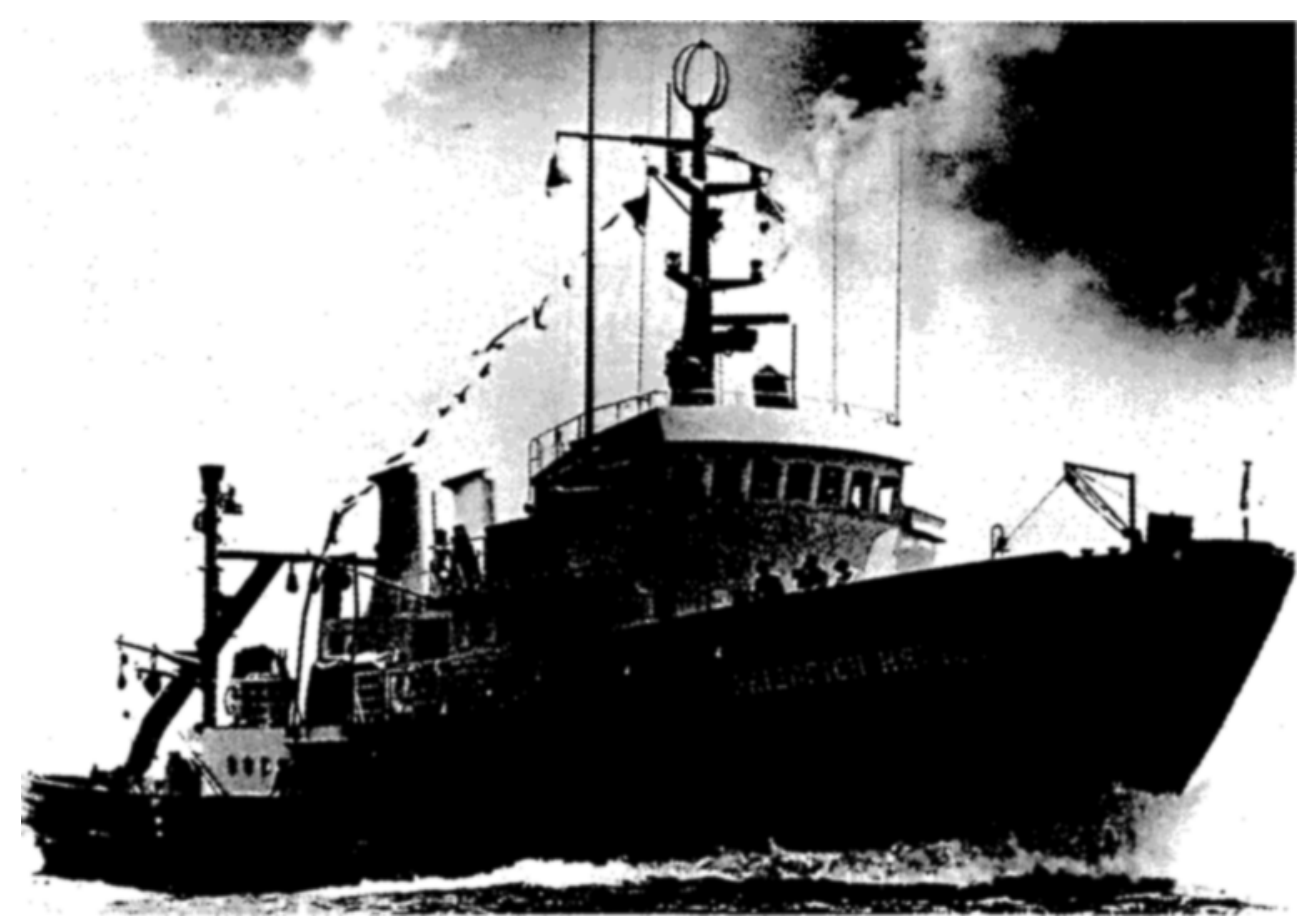

Abb. 23. FK „Friedrich Heincke“, 1968-1990 im Dienste der BAH. Photo: Gerrit Sahling.

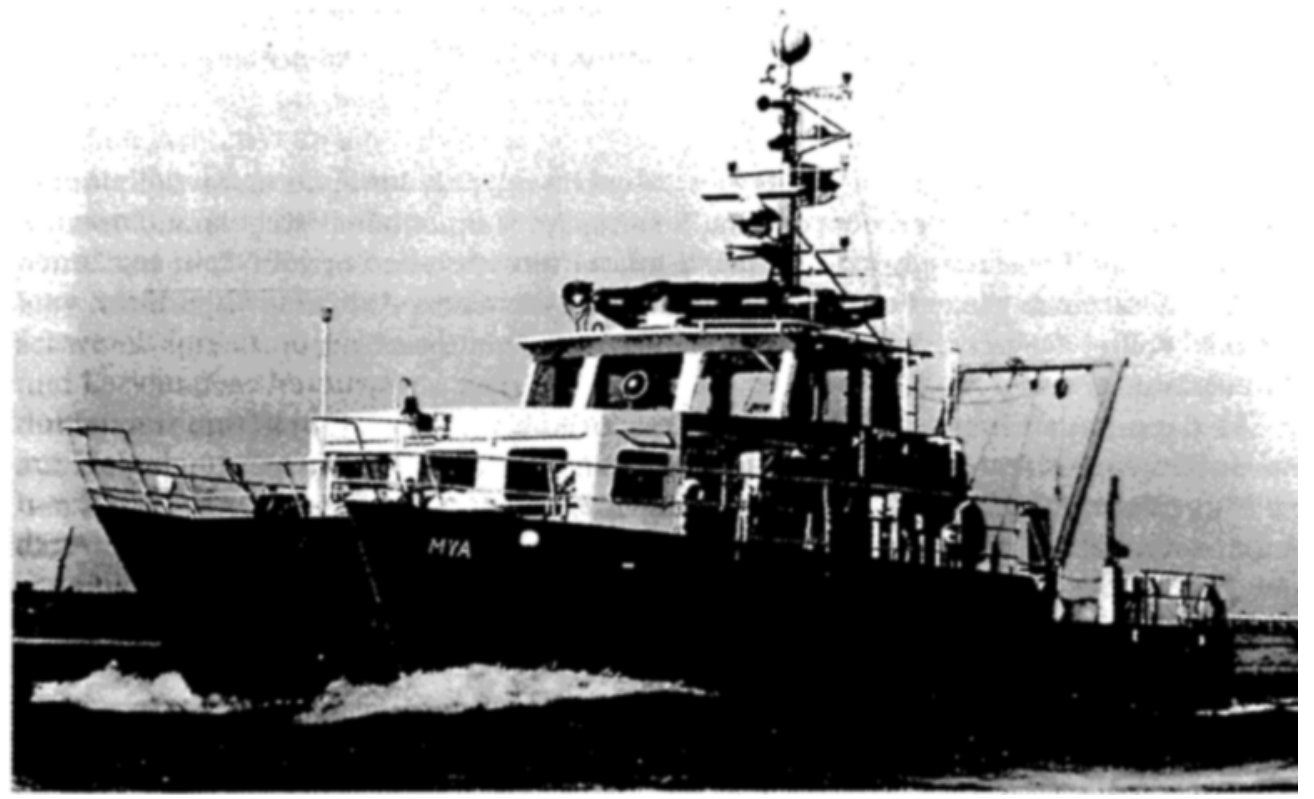

Abb. 24. Forschungskatamaran "Mya” der BAH (ab 1978). Photo: Gerrit Sahling. 
ber 1968 diente das Schiff als Überwasserbasis für die erste Unterwasserstation Deutschlands in der Flensburger Förde. Auf der dritten Fernfahrt wurden fischereiliche Bestandsuntersuchungen durch das Institut für Küsten- und Binnenfischerei in der Nordsee mit Dr. Gert Rauck (Fahrtleitung), Dr. Erdal Aker und Dr. Friedrich Lamp ausgeführt.

Im Bootspark der BAH gab es in den nächsten Jahren folgende Veränderungen: Im Frühjahr 1969 wurde zur Versorgung des "Unterwasserlabors (UWL) Helgoland" ein kräftiges, offenes Arbeitsboot zur Verfügung gestellt, das ursprünglich zur Ringwadenfischerei mit dem Fischereiforschungsschiff "Walter Herwig" bestimmt war. Es war ein 9,9 m langes Aluminiumboot mit einem 350-PS-Motor und, als Spezialausrüstung, KortDüsen-Ruder, Marco-Kran mit Powerblock, Spillkopf, Drahttrommel und Schlepphaken. Bootsführer wurde John ( "Jonny ") Hottendorf. Das Boot, das den Namen des in der Flensburger Förde verunglückten Taucherarztes Horst Hartmann erhielt, war bei der BAH bis 1972 im Dienst.

Ende April 1973 wurde MB "Aquamarin", das ehemalige Verkehrs- und Arbeitsboot des Forschungsschiffes "Valdivia “ neu ausgerüstet, um in List das alte MB "Gelk“ zu ersetzen. Die "Aquamarin“ (9,80 m lang, 3,20 m breit, 0,90 m Tiefgang, 210 PS, $17 \mathrm{kn}$ ), war mit Schiffsführer Adolf Kruse bis zum 18.8.1978 im Einsatz und wurde dann durch den Forschungskatamaran "Mya“ (Abb. 24) abgelöst, später mit den Schiffsführern Niels Kruse und Peter Elvert. Die “Mya" wurde von der Zentralstelle für Schiffs- und Maschinentechnik in Hamburg, die auch "Friedrich Heincke" und die neue "Uthörn" entwarf, nach den Vorstellungen von Dr. Hanswerner Kesseler als Katamaran entwickelt und für 1,5 Mio. DM von der Schiffswerft Evers in Niendorf/Ostsee gebaut. Bei 6,5 m Breite und $17,5 \mathrm{~m}$ Länge stehen ein Arbeitsdeck von $36 \mathrm{~m}^{2}$ und ein Deckslabor von $15 \mathrm{~m}^{2}$ zur Verfügung. Die Möglichkeit, mit dem Schiff auf ebenem Kiel trockenzufallen und der Tiefgang von $0,65 \mathrm{~m}$ sind für die Untersuchungen im Wattenmeer ideal. Durch zwei Dieselmotoren von je 68 PS mit Verstellpropellern kann die "Mya" 8,5 Knoten laufen. Sie hat einen Portalgalgen von $250 \mathrm{~kg}$ Tragfähigkeit und ist auch für Kursausfahrten geeignet.

Auf Helgoland wurde 1967 Werner Krühs, seit 1947 bei der BAH tätig, der Nachfolger von John Herzog auf MB "Ellenbogen". Im Jahr 1971 bekam die "Ellenbogen” ein neues Ruderhaus und eine neue Warmluftheizung. Ein Ersatzbau für das schon über 20 Jahre alte Boot wurde geplant. Nach den Erfahrungen im Inselgebiet wurde ein Holzboot in der bewährten Helgoländer Form auf der Hatecke-Werft in Freiburg/Elbe gebaut (Abb. 25). Es bekam den Namen einer Helgoländer Landmarke, der "Aade" (südöstlicher Dünenausläufer), und konnte am 28. 6.1974 auf Helgoland begrüßt werden. Wieder gab es die traditionellen Kringel für die Kinder. Die "Ellenbogen" wurde noch bis zu ihrer Übergabe an die GKSS Ende Juni 1975 bei Taucheinsätzen und als Vertretung der "Aade" hilfsweise eingesetzt. Am 4. August 1981 wurde für die Tauchgruppe der BAH MB "Diker" (Abb. 25) als neues Taucherarbeitsboot und als Ablösung von MB "Digger“ in Dienst gestellt. Der Name "Diker" stellt wiederum das helgoländische "Taucher" dar, nur in einer durch die Sprachwissenschaft empfohlenen, neuen Schreibweise. Es ist ein 9,2 $\mathrm{m}$ langes und 3,6 $\mathrm{m}$ breites Aluminiumboot mit einem Steuerhaus, in dem sich die Taucher windgeschützt aufhalten und aufwärmen können.

Die "Uthörn” wurde nach der Indienststellung der "Friedrich Heincke“ unter Kapitän Jürgen Stoldt für Tageseinsätze in Fahrt gehalten. In den ersten Jahren gab es allerdings häufig erzwungene Liegetage, weil Besatzungsmitglieder für das unterbesetzte neue Forschungsschiff abgezogen werden mußten. Erst ab 1975 war es nach einer Ver- 


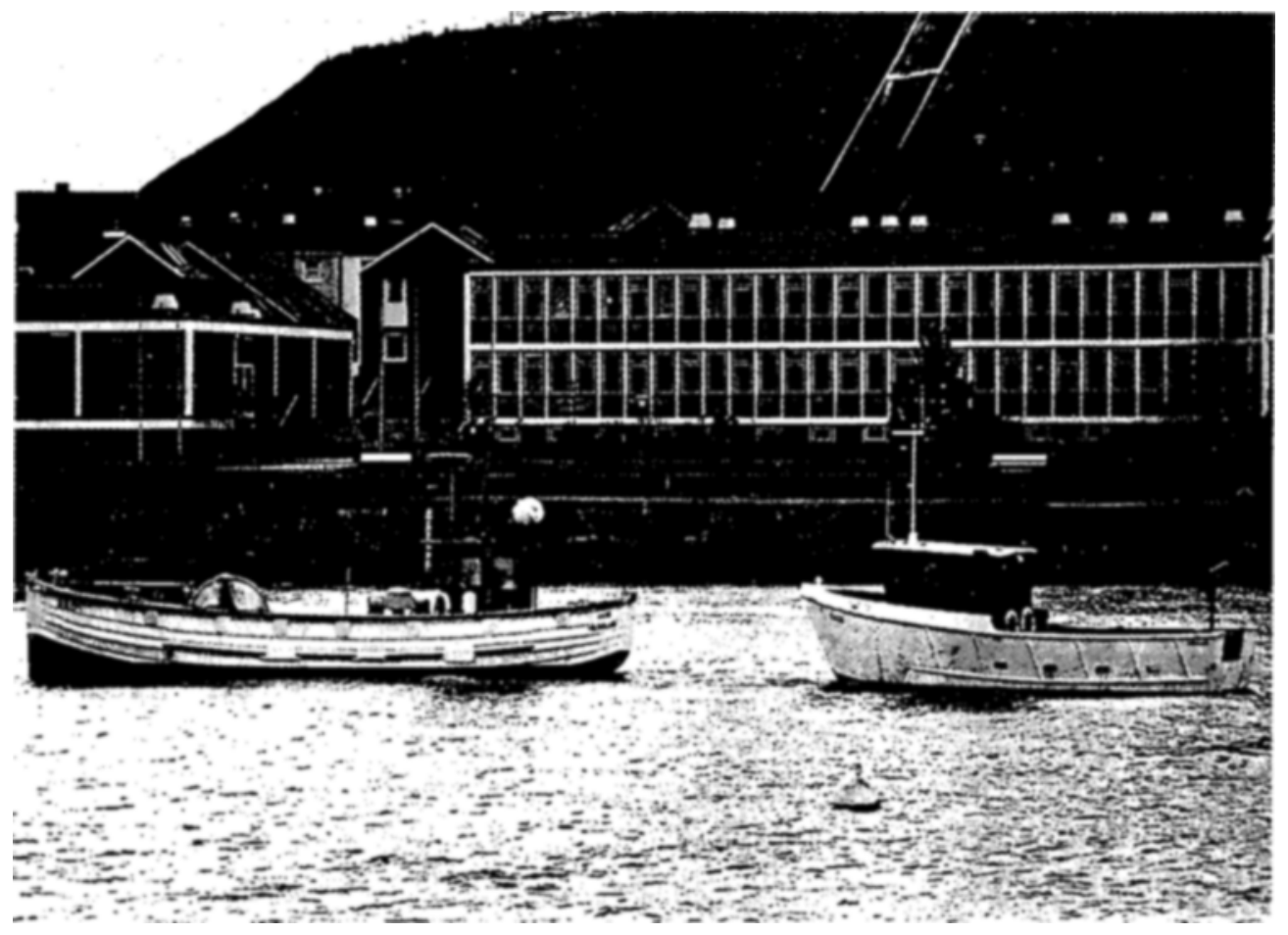

Abb. 25. MB "Aade“ (ab 1974) und MB „Diker” (ab 1981) der BAH. Photo: Traudel Hennemann.

einbarung zwischen der Unterweser-Reederei GmbH (URAG) und der BAH über die Bereitstellung von Seeleuten möglich, durch Personalmangel verursachte Liegezeiten der BAH-Schiffe erheblich abzukürzen. Im Jahr 1970 wurde der Maschinist Peter Singer nach 45jähriger treuer Mitarbeit bei der BAH in einer Feierstunde durch Prof. Kinne verabschiedet. Nachfolger von Peter Singer wurde Gerd Krüß. Die "Uthörn" erhielt Ende 1970 eine neue Antriebsmaschine von 350 PS, zwei neue Brennstofftanks, einen Frischwassertank, einen Schornstein anstelle des Auspuffmastes, eine neue Elektroinstallation und eine neuzeitliche Schallisolierung der Haupt- und Hilfsmaschine. Sie war nach der Modernisierung zeitweise schneller als die "Friedrich Heincke". Untersuchungen über die Aalwanderungen konnten 1973 bis in die Ostsee ausgedehnt werden. Am 1. 10.1975 übergab Kapitän Stoldt die "Uthörn" seinem Steuermann Rudolf Klings.

Am 8. September 1982 wurde ein neuer, auf den Namen "Uthörn“ getaufter Forschungskutter für die BAH in Dienst gestellt, gebaut von der Schiffswerft Gebr. Schlömer in Oldersum/Ems für 6 Mio. DM (Abb. 26). Das Schiff ist 30,5 m lang, 8,5 m breit und hat nur 2,50 m Tiefgang. Es genügt daher der Liegeplatz des alten Kutters im Scheibenhafen auf Helgoland, obwohl die neue "Uthörn" viel größer ist als die Vorgängerin. Zwei Maschinen von je $230 \mathrm{KW}$ treiben Verstellpropeller an, und auch ein Bugstrahlruder ist vorhanden. Es gibt mehrere Spezialwinden statt der einen, universell zu gebrauchenden auf dem alten Schiff. Die über das Heck geholten Netzfänge können auf dem Achterdeck sortiert werden, und zwei Laboratorien, ein Naß- und ein Trockenlabor, gestatten auch 


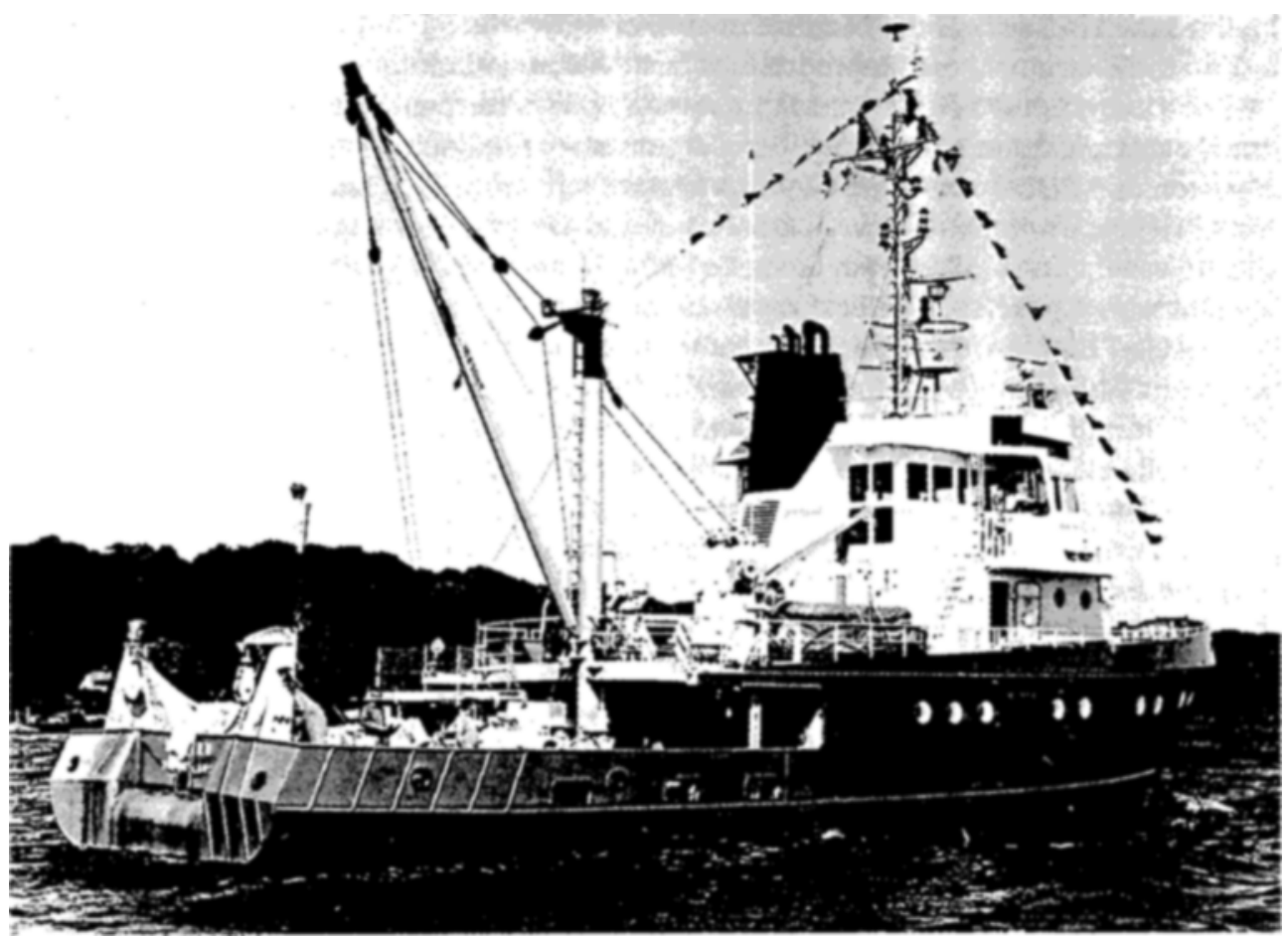

Abb. 26. FK „Uthörn“ der BAH (ab 1982). Photo: Archiv der BAH.

experimentelle Arbeiten an Bord. Bei leicht erhöhten jährlichen Fahrtstrecken blieben die Arbeiten ähnlich vielseitig.

Die „Friedrich Heincke“ absolvierte 1969 insgesamt 39 Fahrten, davon drei längere für die Untersuchung von Grundfisch- und Pandalus-Beständen der Nordsee durch das Institut für Küsten- und Binnenfischerei. Diese Nutzung erfolgte auch in den nächsten Jahren bis zur Indienststeliung des Forschungskutters "Solea" im Jahr 1974 durch die Bundesforschungsanstalt für Fischerei. Im Jahr 1969 wurden nur $9125 \mathrm{sm}$ gefahren, bedingt durch Werftaufenthalte und den Dienst beim Unterwasserlabor "Helgoland". In den nächsten Jahren waren Fahrtstrecken um 13000 sm die Regel. Auf weiteren Reisen in die Biskaya und die Gewässer westlich und südwestlich von Irland untersuchte Dr. Tesch in Zusammenarbeit mit niederländischen Kollegen die Verbreitung von Aallarven und verfolgte in den Atlantik abwandernde Aale.

Am 16. 8. 1974 wurde Kapitän Tönnies Hornsmann in den Ruhestand versetzt, und sein bisheriger Steuermann Hans Falke wurde als Nachfolger eingeführt. Im Jahr 1975 begannen Dr. Wilfried Gunkel und Dr. Günter Gassmann unter internationaler Beteiligung die „Erdölökologischen Nordseefahrten" für Messungen der vorhandenen rezenten und fossilen Kohlenwasserstoffe sowie des Umsatzes durch ölabbauende Organismen. Im Jahr 1977 wurden dabei die Folgen des "Bravo-Blowout" im Ekofisk-Feld untersucht. Im Jahr 1976 war die "Friedrich Heincke" bei den internationalen Untersuchungen der Planktonentwicklung im Fladengrund-Gebiet ( FLEX $76^{\prime \prime}$ ) beteiligt. 
Im Jahr 1979 gab es die bisher längste Fernfahrt von 74 Tagen Dauer in die Sargassosee zur Erkundung der Reproduktion des Aals im Atlantik, zusammen mit dem FFS "Anton Dohrn". Auf beiden Schiffen nahmen BAH-Mitarbeiter teil, wie Dr. Hans Georg Andres (Gammaridenbesiedlung der Sargassum-Vegetation), Dr. Hans-Christian John (Neuston und Fischlarven), Dr. Moebus (Bakteriophagen), Dr. Tesch (Larven und Adulte des Aals) sowie Diplomanden, Doktoranden und Gastforscher aus dem In- und Ausland. Im Jahr 1980 lag die "Friedrich Heincke“ vier Wochen in der Bucht von Galway (Irland) als schwimmendes Labor für Dr. Gassmann und Prof. Dieter G. Müller (Konstanz) für eine biologische und gaschromatographische Untersuchung des Sexual-Pheromons der Braunalge Himanthalia elongata. Sowohl auf der Hin- als auch auf der Rückfahrt fanden Untersuchungen der Sauerstoff-Verhältnisse in der Deutschen Bucht statt. Im Jahr 1981 erfolgte die zweite Fernfahrt zur Untersuchung der Biologie des europäischen Aals in die Sargassosee, und es ergab sich die bisher höchste jährliche Seemeilen-Zahl von 22160. Auch in den folgenden Jahren wurden Laichwanderungen und Orientierung des Aals in längeren Reisen im Atlantik und im westlichen Mittelmeer erforscht. Die Arbeiten über die Lockstoffe von Braunalgen wurden 1981 in der Bucht von Galway und 1983 bei der Isle of Wight mit der Untersuchung von Sargassum muticum fortgesetzt. Seit 1983 wurden von Dr. Hein v. Westernhagen, Dr. Volkert Dethlefsen und Frau Patricia Cameron Planktonfänge in Nord- und Ostsee zur Untersuchung der Mißbildungs- und Schlupfraten pelagischer Fischeier durchgeführt.

Mitarbeiter der BAH nahmen auch an vielen Reisen größerer Forschungsschiffe teil. Die Untersuchungen der Fischereibiologen und Planktologen mit der "Anton Dohrn" wurden schon erwähnt. An Bord der damals neuen „Meteor" arbeiteten während der internationalen Expedition im Indischen Ozean Dr. Gillbricht, Dr. Kotthaus, Dr. Gunkel und Dr. Werner. Fahrten der "Meteor " in den Atlantik wurden für planktologische und meiobenthische Untersuchungen durch Dr. Erik Hagmeier und Dr. Gotram Uhlig genutzt. Mitarbeiter der Taxonomischen Arbeitsgruppe nahmen an vielen Reisen der "Meteor" teil, der "Walter Herwig", der "Anton Dohrn" und später der "Polarstern" bis in die Antarktis. Prof. Gillbricht, Dr. Hagmeier, Dr. Peter Martens und Dr. Hans-Peter Weigel beteiligten sich an der internationalen Fladengrund-Expedition im Frühjahr 1976 (FLEX 76) an Bord von "Anton Dohrn" und "Meteor".

\section{Forschungstaucher, Unterwasserstationen}

Im Jahresbericht für 1965 wird die "Schwimmtauchgruppe" der BAH (Leiter: Dr. Uhlig) zum ersten Mal erwähnt: "Die Anwendung der Methode des wissenschaftlichen Schwimmtauchens hat sich im Rahmen unserer auf Helgoland durchgeführten Untersuchungen sehr bewährt. Die Bearbeitung wichtiger zoologischer und botanischer Fragestellungen ist in Angriff genommen worden."

Angeregt wurde das wissenschaftliche Tauchen durch Prof. Kinne. Die erste Folge war eine Arbeit, die Klaus Lüning als Doktorand des Kieler Meeresbotanikers Prof. Fritz Gessner über die Algenbesiedlung des Helgoländer Felssockels durchführte. Am Anfang schrieb Dr. Erik Hagmeier an Prof. Kinne dazu (14. 3. 1965): „Bei den ersten Tauchversuchen von Herrn Lüning, bei denen wir für seine Sicherheit sorgen sollten, war mir sehr unwohl. Im Ernstfall hätte ein Posten an Land nur wenig ausrichten können. Wir sollten erst weitermachen, wenn von uns noch jemand ausgerüstet und ausgebildet zur Verfü- 
gung steht." So einfach - und gefährlich - begann das Tauchen an der BAH damals. Bald jedoch wurden in Zusammenarbeit mit dem Vizepräsidenten des Verbandes Deutscher Sporttaucher, Karl-Heinz Kerll, mehrere Wissenschaftler und Techniker der BAH als Sporttaucher ausgebildet (Uhlig, 1966). In einem Sonderkurs erhielten Karl-Heinz Schumann und Gerrit Sahling die Abnahmeberechtigung des Tauchsportabzeichens und sorgten so für die Tauchausbildung des wissenschaftlichen Nachwuchses der BAH.

In den Jahresberichten wurden die Vegetationsprofile von Klaus Lüning erwähnt, für die er das Vorkommen von 50 wichtigen Algenarten kartierte. Versuchsalgen zur Registrierung des Wachstums der drei Laminaria-Arten wuchsen im Meer auf 10 „Zuchtstationen", wurden in regelmäßigen Zeitabständen ins Boot geholt, bei konstantem Kamera-Abstand photographiert und wieder ausgesetzt. Eindrucksvoll ist die so gewonnene Darstellung von Laubwechsel und Wachstum der Phylloide von Laminaria hyperborea. Lichtmessungen in situ und die Bestimmung von Assimilation und Atmung im Labor ermöglichten Schätzungen der Primärproduktion der Laminaria-Vegetation.

Die BAH und die Deutsche Forschungs- und Versuchsanstalt für Luft- und Raumfahrt (DFVLR), Institut für Flugmedizin, kamen 1966 überein, die Planung, Aufstellung und Inbetriebnahme eines Unterwasser-Laboratoriums bei Helgoland zu betreiben, das erste in Deutschland. Als erster Einsatzort wurde eine Position $2 \mathrm{sm}$ südöstlich der Helgoländer Düne in etwa $20 \mathrm{~m}$ Tiefe vorgesehen. Die Vorarbeiten umfaßten eine genaue Untersuchung des Standorts, Strömungsmessungen mit Unterstützung des Deutschen Hydrographischen Instituts, eine Ausarbeitung von Methoden zur Orientierung bei schlechten Sichtverhältnissen und die Erprobung eines oberflächen-unabhängigen "Air-Lifts" zur quantitativen Probennahme am Boden.

Im Jahr 1968 bestand die Tauchgruppe aus 14 ausgebildeten Schwimmtauchern, die von Mai bis Dezember 1968218 Tauchgänge bei Helgoland durchführten. Dazu gehörten Untersuchungen zur Ökologie und zum Verhalten des Einsiedlerkrebses (Doktorand Karl-Heinz Schumann) und Beobachtungen der Hummerpopulation (Doktorand Peter Jatzke). Eine lebendige und humorvolle Schilderung vom Leben der Taucher und einiger anderer Mitarbeiter der BAH in diesen Jahren stammt von Peter Lippens (1973).

Das von der BAH und der DFVLR geplante Unterwasserlabor war 1968 beim Drägerwerk in Lübeck unter der Aufsicht von Ing. Fust von der DFVLR im Bau. Die Baukosten von 961000 DM wurden vom Bundesminister für Bildung und Wissenschaft zur Verfügung gestellt. Erste Erfahrungen wurden inzwischen mit der sehr einfach konstruierten Unterwasserstation "BAH I" gewonnen, die Prof. Kinnes Doktorand Gerhard Lauckner konzipiert hatte und die in Zusammenarbeit mit Dr. Dorschel von der Firma Babcock gebaut und der BAH kostenlos zur Verfügung gestellt wurde, eine $6 \mathrm{~m}$ lange Stahlröhre von $2 \mathrm{~m}$ Durchmesser auf vier Spreizbeinen. „BAH I“ wurde als Erste Deutsche Unterwasserstation im Zeitraum 20.9.-1.10. 1968 in $10 \mathrm{~m}$ Wassertiefe in der Flensburger Förde unter der Leitung von Prof. Kinne erprobt, mit der "Friedrich Heincke" als daneben verankerter Versorgungsbasis. Die Hauptschwierigkeiten, die bei ungünstigen Wetterverhältnissen zu meistern waren, lagen an der Wasseroberfläche, durch Wind und Wellen bedingt. Dabei kam es bei einem Tauchgang zum Tod des medizinischen Betreuers der Taucher, Dr. med. Horst Hartmann (DFVLR).

Im Jahr 1969 wurde das größere und technisch ungleich kompliziertere Unterwasserlaboratorium "UWL Helgoland " (Abb. 27) östlich der Helgoländer Düne bei $23 \mathrm{~m}$ Wassertiefe eingesetzt. Die wissenschaftliche Taucheinsatzleitung lag in den Händen von Dr. 


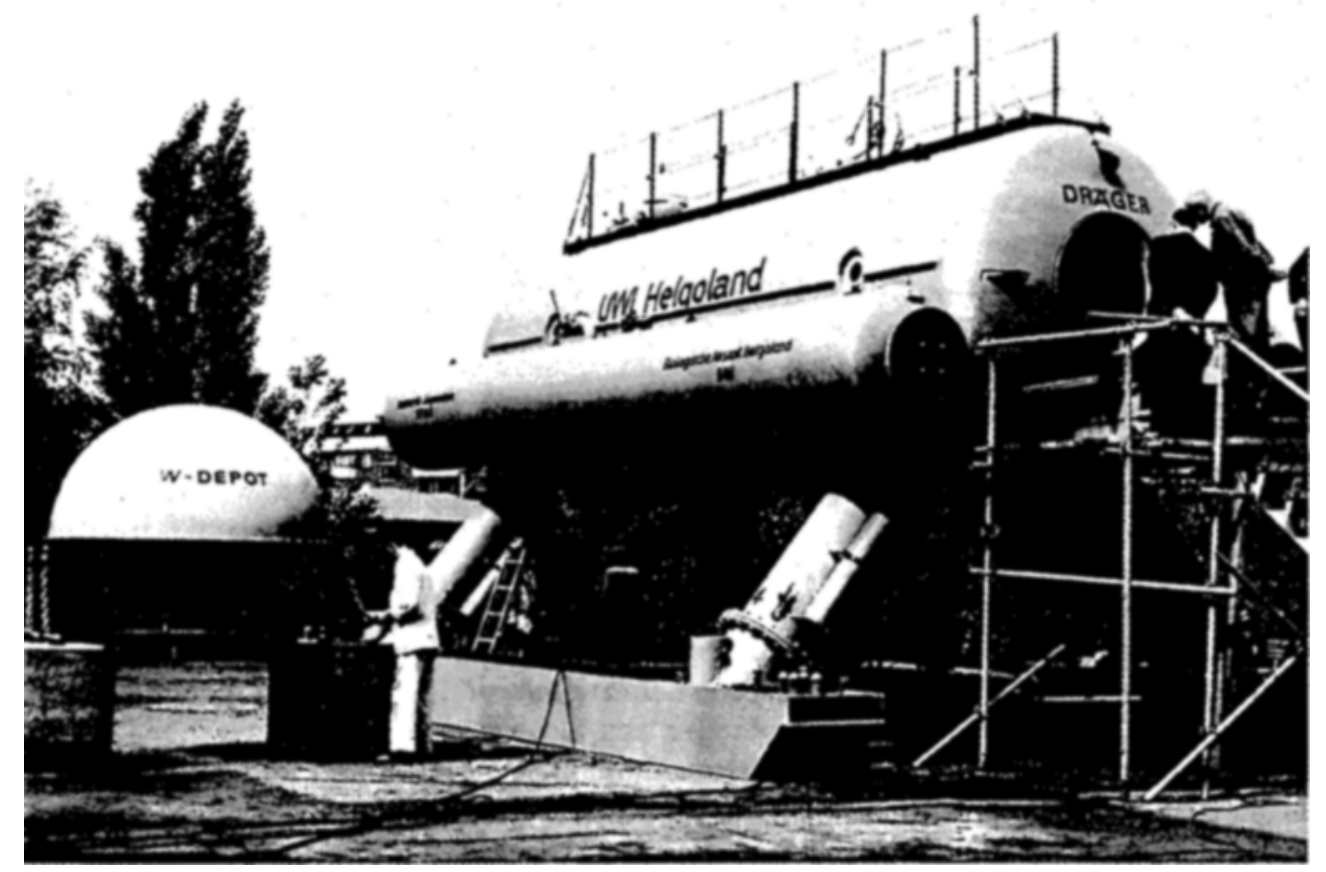

Abb. 27. UWL Helgoland vor dem Ersteinsatz. Links daneben das Unterwasserdepot. Photo: Archiv der BAH.

Uhlig, die technische Leitung bei Ing. Günther Luther. Die Energie- und Gasversorgung des UWL erfolgte von einer großen, an der Wasseroberfläche schwimmenden Tonne aus, die in der Nähe des UWL fest verankert war. Für zahlreiche Sicherheitsvorkehrungen war gesorgt, die medizinische Betreuung erfolgte durch die Tauchärzte Dr. Heinz Oser und Dr. Low (Institut für Flugmedizin). Während des dreiwöchigen Ersteinsatzes vom 28. Juli bis zum 19. August 1969 lebten und arbeiteten die Aquanauten (Abb. 28) Peter Jatzke, Günther Tadday, Dr. Heinz Oser, Dr. Gotram Uhlig, Roland v. Hentig, Karl-Heinz Schumann, Dr. Wolfgang Hickel und H. Platt zwischen 21 und 5 Tagen unterschiedlich lange im UWL. Neben der technischen Erprobung und der medizinischen Kontrolle der Taucher wurden vielerlei Arbeiten ausgeführt, wie Messungen der Temperaturgradienten im bodennahen Wasser sowie im Meeresboden, Messungen von Strömungen, Licht und Trübung in Abhängigkeit vom Tidenstrom, Probennahme für Plankton- und Partikelgehalt während verschiedener Tidenphasen und Auffangen von Sinkstoffen zur anschließenden Analyse. Weiter erfolgten mikrobiologische und mikrofaunistische Untersuchungen zur Ermittlung von Beziehungen zwischen Biota, Wasser und Sediment unter verschiedenen Bewegungsverhältnissen, verhaltensphysiologische Untersuchungen an Hummer und Einsiedlerkrebs sowie Untersuchungen der Möglichkeit, ökologisch und ökonomisch wichtige Meerestiere in Unterwasseranlagen zu züchten und zu mästen. Ausführliche Berichte sind im Jahresbericht der BAH für 1969 zu finden sowie bei Kinne (1970) und Uhlig (1971). 


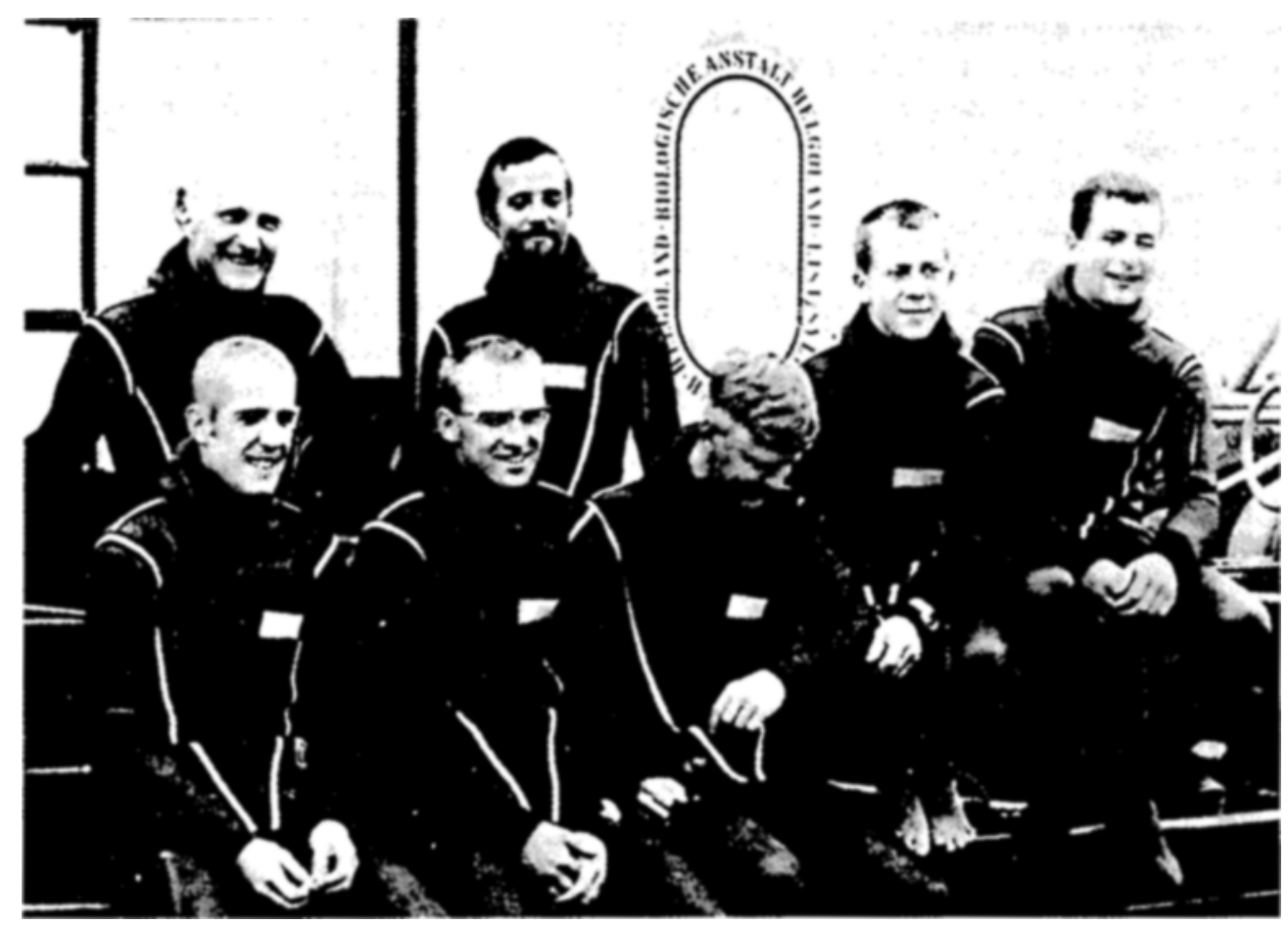

Abb. 28. Die Aquanauten der BAH vom Ersteinsatz des UWL 1969 (ohne H. Platt). Von links nach rechts, stehend. Dr. Gotram Uhlig, Dr. Wolfgang Hickel; sitzend. Dr. Heinz Oser, Karl-Heinz Schumann, Roland v. Hentig, Peter Jatzke, Günther Tadday. Photo: Archiv der BAH.

Nach dem ersten dreiwöchigen Einsatz im Sommer 1969 blieb das UWL am Boden. Damit sollte getestet werden, ob das UWL am Meeresgrund die Herbststürme unbeschadet überstehen konnte. Widrige Wetterbedingungen erlaubten erst im Dezember, eine Bergung des UWL in Angriff zu nehmen. Bei den Arbeiten zur Vorbereitung des Aufschwimmens, die mit Unterstützung einer Fremdfirma durchgeführt wurden, verunglückten die Schwimmtaucher Karl-Heinz Schumann (Doktorand, BAH) und Werner Kreytenberg (Student, Fa. Krause, Kiel) tödlich. Die Ursache des Unfalls konnte nie geklärt werden. Erst im April 1970 wurde das UWL gehoben, und es erfolgten Reparaturen und bauliche Erweiterungen. Für den Betrieb des UWL wurden ein Ingenieur, vier Berufstaucher, zwei Bootsleute, ein Werkstattleiter und ein Elektroniker eingestellt.

Im Jahr 1971 erfolgte eine erneute Erprobung des UWL, dieses Mal in der Eckernförder Bucht. Der große Aufwand für Betreuung und Instandhaltung des Unterwasserlabors führte schließlich im Jahr 1972 zur Überstellung an die Gesellschaft für Kernenergieverwertung in Schiffbau und Schiffahrt (GKSS). Die BAH behielt zwei Berufstaucher, die für die Beschaffung von Lebendmaterial und die Betreuung von Geräten unter Wasser notwendig waren. Im Jahr 1971 begann die Ausbildung von Forschungstauchern auf Helgoland für die BAH und andere Institute.

Das UWL "Helgoland" wurde in der Folge von Instituten der Universitäten Berlin, Clausthal, Göttingen, Hamburg, Kiel und München sowie von vielen ausländischen Wis- 
senschaftlern genutzt und sogar vor der Ostküste der USA eingesetzt. Wissenschaftler der BAH haben im Jahr 1973 bei Helgoland und im Zeitraum 1975-1977 in der Ostsee das Verhalten von Bodentieren und das Plankton im bodennahen Wasser untersucht. Der Schwerpunkt für die Nutzung des UWL verlagerte sich zunehmend in den technischen Bereich. Über einen Einsatz dieser Art in der Ostsee mit dem Mutterschiff "Tabasis" berichteten die "Kieler Nachrichten" am 15.7.1978.

Für die Tauchgruppe unter Tauchermeister Werner Schomburg, seit 1974 verstärkt durch einen Signalmann, gab es regelmäßige Einsätze zur Wartung und Kontrolle von Algenkulturen, von Lichtmeßgeräten und den Sonden zur Messung von Strömungen, Salzgehalt und Temperatur. Die GKSS wurde bei der Erprobung und Wartung der Radionuklidbatterie "Tristan" im UWL-Gebiet südlich der Düneninsel unterstützt. Es gab eine quantitative Bestandsaufnahme von Ascidien im Helgoländer Vor- und Südhafen, Einsätze zur Verfolgung von mit Ultraschall-Sendern markierten Aalen und Hummern sowie jedes Jahr ein umfangreiches Programm zur Beschaffung von biologischem Material für die Untersuchungen der BAH, für Gastforscher, Kurse und für den Versand.

Im Jahr 1981 zerstörte ein Feuer im Hafenlabor die Ausrüstung der Tauchgruppe sowie die Dekompressionskammer, und erst Mitte Dezember 1982 war die "Taucherbasis" im Hafenlabor wieder ganz hergestellt. Im Herbst 1984 fand der 15. Lehrgang für Forschungstaucher statt. Damit waren an der BAH 116 Forschungstaucher ausgebildet worden.

\section{Aquarium und Materialversand}

Aquarien sind wichtig für die Beobachtung der Wasserorganismen und für das Verständnis ihrer Bedürfnisse. Außerdem dienen sie dazu, Erkenntnisse der Meeresforschung einem größeren Kreis von Interessenten zu vermitteln. Das Helgoländer Meeresaquarium wird daher in den Jahresberichten der BAH als Schau-, Lehr- und Versuchsaquarium bezeichnet.

An dem 1901/02 erbauten Aquarium war ein Mäzen entscheidend beteiligt, Herr N. Oppenheim aus Frankfurt am Main. Als regelmäßiger Besucher von Helgoland hatte er gegen Ende der neunziger Jahre angeregt, in Verbindung mit der Biologischen Anstalt ein Schauaquarium zu errichten (Ehrenbaum, 1910). Da der seit der Gründung der BAH geplante Institutsneubau mit einer für die Forschung angemessenen Aquariumsanlage noch nicht abzusehen war, wurden in dem zu bauenden Aquarium auch einige Arbeitszimmer mit kleinen Becken mit besseren Möglichkeiten für Beobachtung. Aufzucht und Experimente als in den bisher verfügbaren Institutsräumen vorgesehen. Das Zentrum dieses ersten Aquariums blieb als "Sortier- und Versandhalle" unter dem neuen Aquarium von 1927 erhalten.

Das erste Helgoländer Aquarium wurde weit bekannt durch das großartige Tafelwerk der Aufnahmen des Photographen Franz Schensky (Königliche Biologische Anstalt auf Helgoland, 1914), das von den Wissenschaftlern der Station mit ausführlichen Kommentaren versehen war. Die Darstellung der Lebensgemeinschaft auf einer Austernbank wurde noch nach dem zweiten Weltkrieg im Museum in Charlottenlund gezeigt. Damals wie heute finden Führungen im Aquarium sowie Gespräche mit den Meeresbiologen "vor Ort" statt. Heute sind die Besucher ungleich umweltbewußter als früher und stellen viele Fragen über das Zusammenleben in der Natur und den Einfluß des Menschen darauf. Studenten, Lehrern und Gästen am Institut werden Beobachtungen und Erfahrun- 
gen bei Hälterung und Vermehrung von Pflanze und Tier vermittelt sowie Einblicke in die dazu benötigte Technik, die auch die Laboratorien versorgt.

Andreas Holtmann, der erste Aquariumsleiter im Neubau von 1959, schreibt von rund 1 Million Besuchern, die in den ersten 8 Jahren nach der Wiedereröffnung kamen (Holtmann, 1967). Bis 1977 lag die jährliche Besucherzahl noch über 100000 , mit einem Maximum im Jahr 1971 von 160024 Besuchern. Neben den bei Helgoland häufigen Tieren und Pflanzen wurden viele Besonderheiten gezeigt. So zog ein Heringsschwarm, der durch internationale Verbindungen zur BAH gekommen war, über drei Jahre im Aquarium seine Kreise. Während einer Untersuchung von Heringsbeständen in der Nordsee kam ein hierfür gechartertes "Markierungsschiff" mit großen Tanks für die vorübergehende Hälterung der Heringe unter Betreuung durch einen norwegischen Kollegen nach Helgoland. Dieses war die überraschende Folge eines wenige Monate zurückliegenden Besuchs von Andreas Holtmann im Saltvandsakwariet Esbjerg, der dort einen Heringsschwarm bewundert und gesagt hatte, wie gerne er so etwas auch in Helgoland zeigen würde. Die "Friedrich Heincke" brachte Krebse, Steinkorallen und Seefedern aus entfernteren Teilen der Nordsee. Ein großer Mondfisch wurde von einem Finkenwerder Kutter gefangen und lebte noch kurze Zeit im Aquarium. Die Dicklippige Meeräsche, sonst ein Fisch an den Küsten von Mittelmeer und Atlantik, erschien nach 61 Jahren wieder bei Helgoland sowie im ostfriesischen Wattenmeer und wurde im Aquarium gezeigt. Tintenfische konnten über ein Jahr gehalten werden, und es gab die Zuchten des Kleingefleckten Katzenhais und der Ohrenqualle.

Ende Mai 1977 ging Andreas Holtmann nach seinem 50jährigen Dienstjubiläum in den"Ruhestand. Sein Vater, der Fischmeister Jacob Holtmann, war über 50 Jahre an der BAH tätig gewesen. Andreas Holtmann wurde nach seinem Mittelschulabschluß 1927 auf Helgoland im Aquarium unter Peter Krüß ausgebildet und beschäftigt. Später arbeitete er mit Dr. Werner an den Versuchen zur Austernkultur in der neuen Station auf dem Ellenbogen. Im Jahr 1945 wurde er auf Betreiben von Dr. Bückmann und Prof. Hagmeier vorzeitig aus der Kriegsgefangenschaft entlassen, so daß er für die BAH am Seefischmarkt Cuxhaven arbeiten konnte. Im Jahr 1947 wurde er zur Kursbetreuung auf dem Ellenbogen abgeordnet und konnte 1948 mit seiner Familie nach List umziehen. Dort hat er u. a. das Motorboot "Ellenbogen“ gefahren, das er 1949 als Neubau von der Kremer-Werft in Elmshorn überführt hatte. Im Jahr 1952 gehörte er mit Paul-Heinz Sahling, Heinrich Herzog und John Herzog, Reimer Rickmers und Hinrich Kanje zu der Arbeitsgruppe der BAH auf Helgoland, die wieder regelmäßig Beobachtungen über Hydrographie und Plankton machte und die für Forschungen und Versand benötigten Pflanzen und Tiere sammelte. Ab 1954 war Andreas Holtmann mit der Planung und dem Bau des neuen Institutsgebäudes auf Helgoland befaßt. Seine Leistungen wurden durch die Verleihung des Bundesverdienstkreuzes gewürdigt.

Nachfolger von Andreas Holtmann als Leiter des Aquariums wurde Olaf Goemann, der Enkel des einstigen Aquariumsverwalters Peter Krüß. Im Krieg auf Helgoland geboren, mußte er 1945 mit der Familie die Insel verlassen und kam 1946 nach List. Dort wurde er durch Großvater und Mutter mit der BAH vertraut und von 1957 bis 1960 als Biologielaborant ausgebildet. Prof. Bückmann, Dr. Hempel, Dr. Ziegelmeier, Dr. Werner und Heinrich Herzog sorgten für eine gründliche zoologische Schulung an Land und auf See. Seine erste Anstellung erfolgte in der Abteilung Fischereibiologie, und nach der Wehrdienstzeit kam er zur Arbeitsgruppe Materialversorgung. Im Jahr 1974 wurde er als 
Nachfolger von Heinrich Herzog der Leiter der Materialversorgung und blieb es auch nach der Emennung zum Aquariumsleiter.

Der BAH-Jahresbericht für 1977 hielt fest: „Wieder wurde ein Seehund geboren. Bei einem Bestand von nunmehr acht Seehunden wird die Abgabe von einigen Tieren notwendig". In den folgenden Jahren wurden Schauraum und Eingangshalle mit Plakaten und Exponaten über die Arbeiten des Instituts informativer gestaltet. In zunehmendem Maße wurden Aufnahmen von Fernsehteams gemacht. Helgoländer Schüler kamen im Rahmen ihres "Berufspraktikums" bevorzugt in das Aquarium. Es gab erfreuliche Ergebnisse bei der Anzucht von Nagelrochen, Seehasen, Katzenhaien, Bastardmakrelen, Tintenfischen und Aalmuttern. Daneben wurde aber auch von langwierigen und umfangreichen Reparatur- sowie Umbaumaßnahmen berichtet. Im Jahr 1982 sah der Aquariumsbereich wie eine Großbaustelle aus, und das Aquarium mußte häufig geschlossen werden. Dennoch wurden im Jahr 1982 noch 60302 Besucher gezählt.

Olaf Goemann beschäftigte sich auch eingehend mit dem Hummer. Im Aquarium wurden zahlreiche Präparate und ein Modell der Fangmethode ausgestellt. In einem Büchlein (Goemann, 1990a) wurden Beobachtungen, Studien und Versuche zur Häutung, Vermehrung, Aufzucht und Orientierung des Hummers zusammengetragen. In einer weiteren Arbeit (Goemann, 1990b) wurden Hintergründe und Theorien zum Rückgang des Hummerbestandes bei Helgoland untersucht. Um den Nachwuchs zu erhalten, verkauften die Helgoländer Fischer seit 1983 eiertragende Hummerweibchen nicht mehr, sondern brachten sie zum Aquarium. Dort wurden die ersten Entwicklungsstadien versorgt, und jährlich wurden zwischen 10000 und 400000 Larven im Felssockelgebiet wieder ausgesetzt.

Eng mit dem Aquarium verbunden ist die "Versandstelle zur Versorgung von Lehrund Forschungseinrichtungen mit maritimem Untersuchungs- und Lehrmaterial ", die seit Bestehen der BAH intensiv genutzt wurde. Prof. Heincke schrieb in seinem Jahresbericht für 1893: „Der Versand von lebendem und konserviertem Untersuchungsmaterial für wissenschaftliche Zwecke ist eine der Hauptaufgaben der Anstalt " und zählte die Museen, zoologischen und botanischen Institute sowie die Aquarien im In- und Ausland auf, an die schon Material verschickt wurde (Heincke, 1894).

Nach dem Zweiten Weltkrieg mußte das Angebot reduziert werden, bis Helgoland wieder zugänglich war. Die von der BAH versorgten Universitätsinstitute wußten aber auch das noch Erreichbare sehr zu schätzen. Im neuen Gebäude auf Helgoland gab es wieder ausreichend Platz zum Sortieren, Präparieren und Aufbewahren. Im Jahresbericht für 1966 wurde erwähnt, daß 59 inländische und 12 ausländische Institutionen auf dem Festland versorgt wurden. Der Leiter des Materialversands, Heinrich Herzog, hatte dafür zwei Mitarbeiter, Gerda Duve und Olaf Goemann. In den folgenden Jahren stieg die Anzahl der Lieferungen, und es wurde von Engpässen bei der Beschaffung der gewünschten Tiere berichtet, von Sendungen bis in die USA und nach Australien, aber auch, daß der Lebendversand ins Ausland wegen zeitaufwendiger Zollbehandlung im Jahr 1972 noch nicht erfolgen konnte.

Am 30. April 1974 wurde Heinrich Herzog nach 50jähriger Tätigkeit bei der BAH wegen Erreichens der Altersgrenze feierlich aus dem Dienst verabschiedet, und Prof. Kinne überreichte ihm im Auftrage des Bundespräsidenten das Bundesverdienstkreuz. Olaf Goemann übernahm die Leitung der Materialversorgung. Im Jahr 1977 kam es zu einer Personalunion zwischen Aquarium und Versand bei Einsparung einer Stelle. Für 1980 
wurden auch im Jahresbericht Verzögerungen durch Personalmangel beklagt, zumal die Mitarbeiter zahlreiche Nebenaufgaben wie Transporte, Ausgabe von Chemikalien und die Versorgung von Gastforschern und Kursen übernehmen mußten. Noch unangenehmer war der starke Rückgang der Sandigel-(Psammechinus)-Bestände bei Helgoland und im Wattenmeer. Jahrzehntelang war der Sandigel ein zuverlässiges Objekt für die Beobachtung von Furchung und ersten Larvalstadien gewesen. Bei Lebendversand der geschlechtsreifen Tiere war dieses auch in Kursen auf dem Festland möglich. Es wurden auch konservierte Entwicklungsreihen verschickt. Eine Erklärung für den Rückgang der Sandigel-Bestände gibt es noch nicht, jedoch stehen in den letzten Jahren wieder einige Psammechinus-Exemplare zur Verfügung.

\section{6. Öffentlichkeitsarbeit}

Der Wunsch nach einem Platz zur Unterbringung einer Sammlung von Pflanzen und Tieren der Nordsee, die auch für das Publikum zugänglich sein sollte, und dessen Dringlichkeit wurde schon im Jahresbericht des ersten Direktors (Heincke, 1894) ausgedrückt. Hier erschien bereits der Begriff "Nordseemuseum". Im Sommer 1897 konnte das Museum im ehemaligen Konversationshaus der Gemeinde Helgoland eröffnet werden, wobei die Einrichtung durch eine großherzige Spende der Erben Nathanael Pringsheims ermöglicht wurde (Heincke, 1897).

Die erste größere Darstellung der Biologischen Anstalt auf dem Festland gab es anläßlich der deutschen Seefischerei-Ausstellung in Berlin 1896. Dabei leitete Dr. Ehrenbaum als Kommissar der Ausstellung Organisation und Aufstellung des Teiles, der die Vorführung des praktischen Betriebes der Seefischerei und die Fischkosthalle umfaßte. Prof. Heincke organisierte die wissenschaftliche Abteilung mit Beiträgen verschiedener Museen und der Sammlung nutzbarer Seetiere des Seefischerei-Vereins. Die BAH war dabei am stärksten vertreten. Prof. Heincke (1897) schrieb dazu: „Besonders erwähnenswert waren aus dieser unserer Sammlung die Präparate zur Naturgeschichte des Hummers, die Entwicklung und Wachstum desselben vom Ei bis zum erwachsenen Tiere veranschaulichten, ferner schöne Reihen ganz junger Plattfische, die die Entwicklung derselben von der symmetrischen Larve bis zur Ausbildung der Asymmetrie und das weitere Wachstum bis zur Geschlechtsreife vorführten, endlich ausgesucht schöne Exemplare zahlreicher Nutzfische der Nordsee, wie Kabeljaue, Schellfische, Schollen, Seezungen u.a., die fast alle auf eine neue Art mit ausgebreiteten Flossen konserviert und in natürlicher Stellung, zum Teil auf natürlichem Grunde, aufgestellt waren. Von manchen der wichtigsten Nutzfische, z. B. dem Schellfisch und der Scholle, wurden von uns auch Grundproben von den wichtigsten Nährgründen derselben, der Mageninhalt und die vornehmsten Nährtiere in übersichtlicher und belehrender Weise ausgestellt.“

Ein Beitrag für die große Zahl der Gäste an unseren Küsten gelang Prof. Kuckuck, dem damaligen Botaniker der BAH, mit dem "Strandwanderer" (Kuckuck, 1905; 1. Auflage), einem mit farbigen Tafeln des Porzellanmalers Julius Braune (1879-1903) ausgestatteten Büchlein für die erste Bekanntschaft mit den Pflanzen und Tieren und ihrer Lebensweise in und am Meer. Nach dem Tode Prof. Kuckucks wurde der Strandwanderer seit der 3. Auflage durch Mitarbeiter der BAH herausgegeben, auf den neuesten Wissensstand gebracht und ergänzt, zum Beispiel um die Vögel unserer Küsten. Nach der 10. Auflage wurde die weitere Bearbeitung durch die BAH abgelehnt, da man an die 
Herausgabe eines mehrbändigen Werkes über Flora und Fauna der Deutschen Bucht dachte. Berliner Kollegen besorgten eine 11. Auflage (Kuckuck, 1974), da sie die Konzeption des "Strandwanderer" auch weiterhin für Studenten und Biologielehrer als nützlich empfanden. Die vertrauten Tafeln mußten dabei ersetzt werden, und der Text wurde erweitert.

Eine informative, vielseitige und humorvolle Darstellung von Mensch, Natur und Schiffahrt an der Deutschen Bucht brachte Kuckuck mit dem "Nordseelotsen“ (Kuckuck, 1908) heraus, illustriert durch Scherenschnitte und Skizzen von Helene Varges und Margarete Boie. Eine zweite, stark erweiterte Auflage (Kuckuck, 1924) wurde von der BAH besorgt. Beide Ausgaben sind wertvolle Zeugen der früheren, jetzt stark veränderten Verhältnisse. Man findet im "Nordseelotsen" zum Beispiel eingehende Beschreibungen der Becken im alten Aquarium und der Ausstellung im Nordseemuseum. Eine erneute Bearbeitung, auf die Vergangenheit bezogen und die Gegenwart beschreibend, würde auch heute noch einen dankbaren Leserkreis finden.

"Dieser Reichtum unseres Aquariums an schönen und seltsamen Formen und an interessanten Lebens-Momenten aus der Tier- und Pflanzenwelt hatte schon lange den Wunsch in uns erweckt, einzelne dieser Formen und Momente im Bilde festzuhalten und sie dadurch auch weiteren Kreisen anschaulich vorzuführen. Daß dieser Wunsch jetzt zur Wirklichkeit geworden ist, verdanken wir dem glücklichen Umstande, daß wir in Herrn Hofphotographen Schensky in Helgoland einen begeisterten und technisch wie künstlerisch gleich hervorragenden Helfer gefunden haben". So schrieb Prof. Heincke im Vorwort zum umfangreichen Tafelwerk "Tier- und Pflanzenleben der Nordsee", das in 1. Auflage im Jahr 1914 erschien. Texte der Mitarbeiter der BAH ergänzten die Aufnahmen mit für Laien gut verständlichen, auch heute noch aktuellen Beschreibungen und Beobachtungen von Leben und Zusammenleben.

Noch bevor im Jahr 1920 geeignete Räume die Unterrichtung von Studierenden und Lehrern möglich machten, „hat die Anstalt regelmäßig kleine Kurse für Ärzte und Offiziere der Fischerei-Aufsichts- und der Vermessungsfahrzeuge der Kaiserlichen Marine und gelegentlich auch für Fischereibeamte abgehalten" (Heincke, 1918). Mittelpunkte zur Ansprache eines größeren Publikums blieben zwischen den beiden Kriegen Museum und Aquarium, in dem von den Wissenschaftlern der BAH viele Führungen veranstaltet wurden. Dazu kamen allgemein verständliche, meistens mit den einzigartigen SchenskyPhotos illustrierte Aufsätze, die zum Teil eine weite Verbreitung fanden, wie "Meeresleben. Aus dem Aquarium der Biologischen Anstalt auf Helgoland " (Hagmeier, 1934) oder "Helgoland und die Biologische Anstalt" (Hagmeier, 1952). Prof. Kinne setzte diese Tradition mit ähnlichen Beiträgen fort (Kinne, 1965, 1967a, 1967b, 1980). Ab Mitte der 60er Jahre wurden aufgrund einer Vereinbarung zwischen Prof. Kinne und dem Helgoländer Bürgermeister und Kurdirektor Henry Peter Rickmers für Gäste und Einheimische regelmäßig Lichtbilder-Vorträge und Diskussionen zum Thema "Das Meer bei Helgoland und seine Bewohner" von einem Wissenschaftler der BAH gehalten, im Zeitraum 1967-1987 meistens von Dr. Erik Hagmeier.

Bei der "Interocean '70", Internationaler Kongress und Ausstellung für Meeresforschung und Meeresnutzung in Düsseldorf 1970, war die Biologische Anstalt vertreten durch eine Darstellung der ökologischen Beziehungen zwischen den bei uns häufigen Arten (Dr. Greve) und durch ein großes, 16000 Liter fassendes Aquarium (Konstruktion J. K. Holtmann, Sohn von Andreas Holtmann), in dem sich Dorsche, Schollen, Steinbutt, 
Rochen und Helgoländer Hummer wohlfühlten. Dieses Becken wurde übrigens noch bis 1992 im Ökolabor auf Helgoland zu vielen Versuchen und Zuchten ( BAHMAPLEX”) gebraucht.

Wie oben erwähnt, wurden ab 1978 Schauraum und Eingangshalle des Aquariums mit Plakaten und Exponaten über die Arbeiten der BAH informativer gestaltet. In zunehmendem Maße kamen Fernsehteams, und Helgoländer Schüler arbeiteten im Rahmen des Berufspraktikums bevorzugt im Aquarium. Seit 1982 gibt es die Rubrik "Öffentlichkeitsarbeit" in den Jahresberichten der BAH. Im Jahr 1983 wurden von Olaf Goemann Schülerarbeitsbögen mit dem Thema "Unterricht im Aquarium Helgoland" entwickelt , die von Lehrkräften und Biologiekursen intensiv genutzt wurden. Auf dieser Grundlage entstand fünf Jahre später in der Zeitschrift "Seevögel “ das Heft "Helgoland - Eine Arbeitshilfe zum Kennenlernen eines einzigartigen Naturraumes" von Eckhard Bockwoldt und Olaf Goemann.

\section{Gastforscher, Kurse und Studenten}

Die BAH verdankt den Gastforschern wertvolle Beiträge zur Kenntnis der Fauna und Flora bei Helgoland und Sylt. So erschien in Band 1 der Wissenschaftlichen Meeresuntersuchungen, Abteilung Helgoland, eine Arbeit von R. Timm aus Hamburg über die Ruderfußkrebse des Helgoländer Planktons (Timm, 1894). R. Lauterborn aus Ludwigshafen beschrieb die Protozoen und Rotatorien (Lauterborn, 1894), und A. Scherffel gab den im Mai bei Helgoland in Massen vorkommenden Algenkolonien 1899 den Namen Phaeocystis globosa (Scherffel, 1899). Diese Alge färbt in jedem Jahr an den Küsten von Belgien bis Norwegen das Wasser braun, und wenn sie am Strand zersetzt wird, berichtet die Presse von ihr als "Schaumalge". Hubert Caspers beschrieb die Lebensgemeinschaften am Boden der Helgoländer Tiefen Rinne (Caspers, 1938) und der früheren Austernbank östlich der Insel (Caspers, 1950). Die Auswirkungen des kalten Winters 1962/63 auf die Helgoländer Branchiostoma-Population wurden von W. A. M. Courtney und J. E. Webb (1964) untersucht. Die Polychaetenfauna des Sylter Litorals bearbeitete Wilfried Westheide (1966), die des Helgoländer Felswatts Lisel Gillandt (1979). Klaus Janke schrieb seine Diplomarbeit über die Makrofauna und ihre Verteilung im Felswatt von Helgoland (Janke, 1986) und promovierte 1989 über die dort gefundenen biologischen Wechselbeziehungen. Zusammen mit dem Kölner Botaniker B. P. Kremer (Gastforscher seit 1970 und später auch Kursleiter auf Helgoland) gab er für naturwissenschaftlich interessierte Besucher eine sehr anschauliche Beschreibung Helgolands (2. Auflage 1990). Schließlich hat der auf Helgoland geborene Andreas Krüß als Gastforscher an der Biologischen Anstalt eine gründliche Diplomarbeit über die Fische des Helgoländer Felssockels verfaßt (1988).

Für Gastforscher waren 1959 an der Meeresstation Helgoland im Neubauplan für Gäste acht allgemein-biologische und drei spezielle Labors für Mikrobiologie, Chemie und Enzymchemie ausgewiesen. Im Jahresbericht für 1968 war zu lesen: „Die Anzahl der jährlich durch die BAH betreuten Gastforscher, Kursleiter und Studenten wächst. Immer häufiger können Anmeldungen nicht mehr berücksichtigt oder Gäste auf Helgoland und Sylt nicht angemessen betreut werden." Nach zahlreichen Beratungen mit Vertretern der Ministerien, der DFG und mit Wissenschaftlern der interessierten Hochschul- und MaxPlanck-Institute sprach sich 1970 der Senat der DFG für eine Förderung der Gastforschung an der BAH aus. Mit den 1971 bewilligten DFG-Mitteln wurden Speziallabors für 
Biochemie und Stoffwechselphysiologie (Federführung: Prof. Karlson, Marburg), Elektrophysiologie (Prof. Hensel, Marburg), Photobiologie (Prof. Nultsch, Marburg) sowie Histologie (Prof. Siewing, Erlangen) eingerichtet. Zum DFG-finanzierten Betreuungspersonal unter Leitung von Dr. Wulf Greve gehörten eine technische Assistentin, eine Schreibkraft, ein Ingenieur mit Elektronikwerkstatt sowie ein Feinmechaniker zur Wartung der wissenschaftlichen Geräte, weiter ein Bootsführer, ein Maschinist und ein Matrose zur Materialversorgung.

Eine Broschüre mit einem Titelblatt mit Inselmotiv (Abb. 29) stellte den Gastforschern Einrichtungen und Arbeiten an der Meeresstation und die typischen Vertreter der verfügbaren Meeresflora und -fauna vor und informierte über die technischen Einzelheiten eines Inselaufenthalts.

Im Jahresbericht für 1972 wurden die Räume für Photobiologie und für Biochemie mit ihren Apparaten beschrieben und die Nutzung der 10 Gastforscherlabors an der Meeresstation dargestellt, die schwerpunktmäßig in den Monaten Juni bis September stattfand. Einer dieser Räume konnte für kinematische Forschungsarbeiten ausgerüstet werden und wurde "Willi-Kuhl-Laboratorium" benannt. Professor Wilhelm Kuhl aus Frankfurt hatte schon seit 1922 und ab 1932 mit seiner Ehefrau Gertrud (1908-1967) zusammen fast alljährlich an der BAH gearbeitet, Entwicklung, Wachstum und Bewegung mariner Organismen untersucht und in Filmdokumenten festgehalten. Die Kinematographie wurde von den beiden Wissenschaftlern dabei gezielt eingesetzt und entwickelt, um für normale Beobachtungen zu schnell oder zu langsam ablaufende Vorgänge zu registrieren und zu analysieren. Vor seinem Tode übereignete Prof. Kuhl seine Geräte der BAH in freundschaftlicher Verbundenheit. Er starb Ende 1972.

Ein weiteres Beispiel für einen treuen Gastforscher ist Dr. Karl Herrmann. Er begann 1972 als Student aus Erlangen mit Untersuchungen über eine bizarre Larve, die schon 1845 den berühmten Berliner Zoologen und Mediziner Johannes Müller im Helgoländer Sommerplankton begeistert hatte. Sie wird nach ihrer planktischen Zeit zu dem unscheinbaren, in weichem Boden stellenweise sehr häufigen wurmartigen Tentakulaten Phoronis. Karl Herrmann promovierte über die Metamorphose von Phoronis und entdeckte, daß Mikroorganismen im Meeresboden den Larven die richtige Stelle zum Siedeln signalisieren. In der Folge kam Dr. Herrmann fast in jedem Sommer zu Untersuchungen der Entwicklungsgeschichte planktischer Larven nach Helgoland.

Für Kurse gibt es in der Wattenmeerstation List einen großen Arbeitsraum mit 20 Plätzen und in der Meeresstation Helgoland zwei Kursräume, mit 25 oder 20 Plätzen, jeweils mit den nötigen Mikroskopen, Glasgeräten und Aquarien. In jedem Jahr kommen nach Helgoland oder List Kurse mit Studenten und Kursleitern von fast allen deutschen und einigen ausländischen Universitäten, viele über eine lange Reihe von Jahren. Die Kursleiter sind dann mit Flora und Fauna bei Helgoland oder Sylt sowie der Umwelt der Inseln vertraut, und oft entstehen gehaltvolle Protokolle mit Berichten, Fundlisten und Ausarbeitungen.

Beispiele für besonders langjährige Kursleiter von Kursen auf Helgoland sind die Tübinger Botaniker Prof. Berthold Schwemmle von 1964 bis 1988 und Dr. Klaus Drumm von 1964 bis 1990 sowie Dr. Erich Zimmermann vom Botanischen Institut der Universität Marburg, der schon 1968 mit Prof. Nultsch nach Helgoland kam und seit 1971 die Marburger Kurse leitet, wobei die Botanik durch einen zoologischen Mitarbeiter ergänzt und ein ökologischer Einblick angestrebt wird. Weitere Beispiele sind Dr. Manfred Kraml vom 


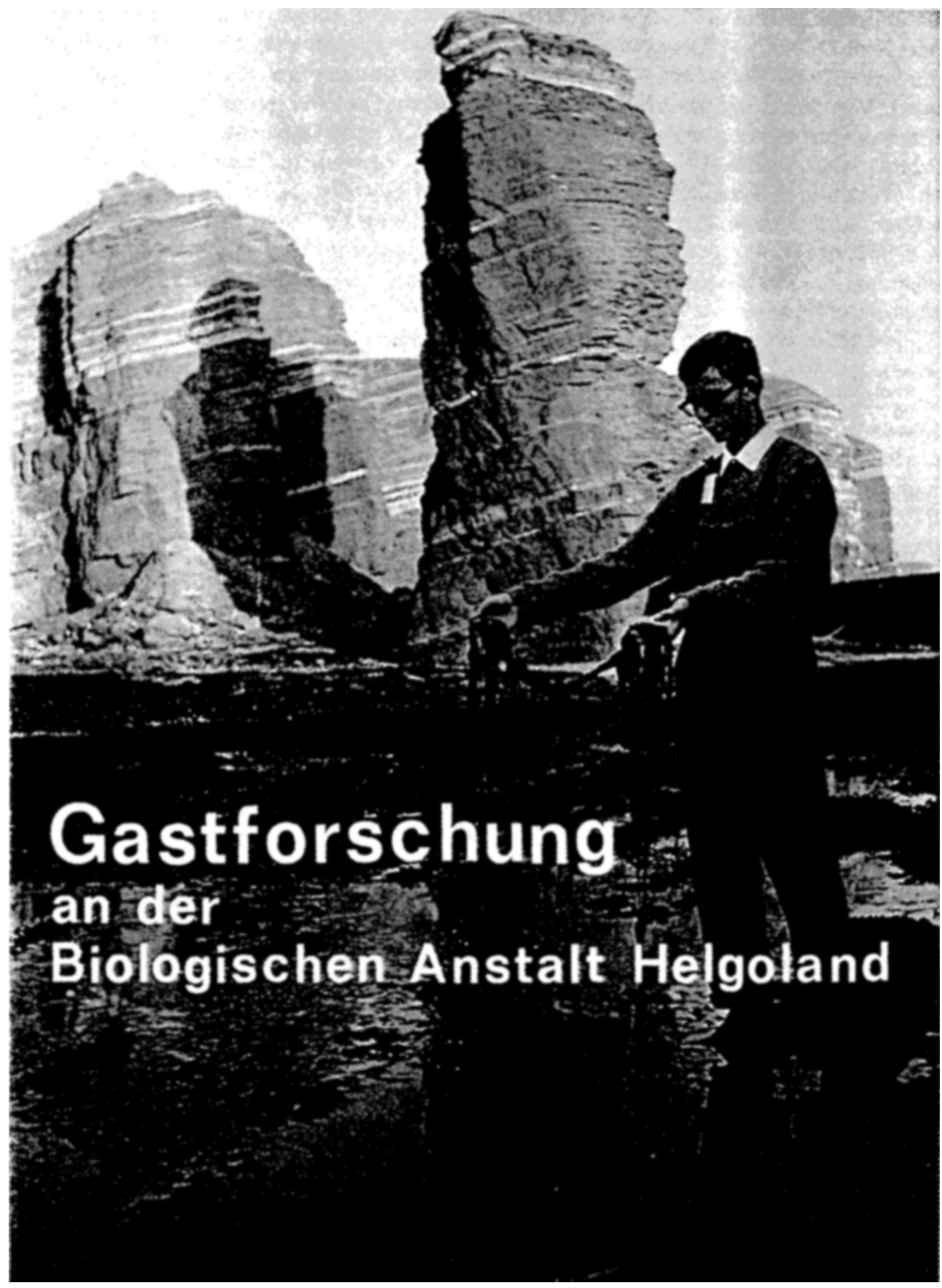

Abb. 29. Titelseite der ersten Broschüre für Gastforscher (etwa 1971), mit Techn. Ass. Rainer Lüdtke im Felswatt vor der "Langen Anna”. Photo: Paul-Heinz Sahling. 
Botanischen Institut der Universität Erlangen-Nürnberg, ebenso mit Zoologen, seit 1969, oder Prof. Dr. Ludwig Kies vom Institut für Allgemeine Botanik der Universität Hamburg. der seit 1972 mit Studenten nach Helgoland kommt und mit Prof. Klaus Lüning den Kurs leitet. Von den Zoologen kommen wohl am längsten mit Kursen an die BAH: Prof. Dr. Klaus-Jürgen Götting (früherer Mitarbeiter der BAH, dann Universität Gießen) seit 1968, Prof. Dr. Otto Larink vom Zoologischen Institut der Universität Braunschweig seit 1971, Dr. Wolfgang Weidemann von der Tierärztlichen Hochschule Hannover seit 1972, Dr. Rainer Bergfeld vom Zoologischen Institut der Universität Freiburg von 1972 bis 1991, seit 1977 mit Dr. Dieter Zissler, der seit 1992 die Freiburger Kurse leitet und ein Mitherausgeber des Buches "Meeresbiologische Exkursion" ist, das viele Beiträge von BAHMitarbeitern enthält (Emschermann et. al., 1992).

Vor 1968 war die Unterbringung der Kursteilnehmer in der Jugendherberge und den wenigen freien Zimmern des Gästehauses nicht ideal. Es war eine große Erleichterung, als 1968 das neue "Wilhelm-Mielck-Haus“ bezogen werden konnte, ein moderner Atrium-Bau auf dem „Nordost-Gelände“ (Abb. 30). Eine Bewilligung der Mittel durch das Ministerium wäre nicht möglich gewesen, denn für größere Aufwendungen im Rahmen des Stationsbetriebes war der Träger der BAH nicht zuständig. Prof. Kinne berichtete darüber in der Ansprache bei seiner Ernennung zum Präsidenten der NathanaelPringsheim-Gesellschaft (Kinne, 1992): „Nachdem durch Absprachen mit der Gemeinde Helgoland und der zuständigen Bundesinstanz ein Grundstück bereitgestellt werden konnte, warb ich um die Unterstützung namhafter Zoologen und Botaniker. Das Ergebnis war ein Antrag an die Stiftung Volkswagenwerk zur Baufinanzierung. Die Stiftung war meinem Antrag gegenüber aufgeschlossen. Wer aber sollte für die Folgekosten aufkommen? Die VW-Stiftung hatte da gerade böse Erfahrungen gemacht und bestand daher auf einer Vorabklärung dieser Frage. Aber der damals zuständige Träger (das BML) konnte beim besten Willen die Folgekosten für ein Studentenheim nicht übernehmen. Schließlich gelang es mir, einen schleswig-holsteinischen Verein als Betreiber des Studentenheimes zu gewinnen. Verständlicherweise wollte der Verein das Heim auch selber für die von ihm betreuten Jugendlichen nutzen. Ein entsprechendes Abkommen wurde unterzeichnet. Das alles lief gut, bis der Bau fertig war. Dann aber lagen mir bereits so viele Kursanmeldungen vor, daß für die Nutzung durch den Verein praktisch keine Zeit mehr übrig blieb, außer im tiefen Winter - und wer will dann schon nach Helgoland? Ich teilte diese Entwicklung dem Verein mit. Es gab Enttäuschung und Verärgerung. Der Verein zog sich zurück. Nun aber stand der Übergabetermin des Neubaus vor der Tür und kein Mensch wußte, wem denn nun eigentlich das "Mielck-Haus“ übergeben werden sollte. So kam was kommen mußte: Ich war Bauherr, und so wurde das Heim mir persönlich übergeben. Formal war ich Eigentümer und blieb es eine ganze Weile. Verschlungene Wege in der Tat und bemerkenswerte dazu!" Am 3. August 1968 wurde das "Wilhelm Mielck Haus" eingeweiht. Es wird seitdem vom Heimleiterehepaar Dieter und Bärbel Pflaumbaum versorgt. In den ersten 10 Jahren wohnten darin 8174 Gäste aus dem In- und Ausland mit 64905 Übernachtungen.

Mitarbeiter der BAH unterrichten jedes Jahr in einem allgemeinen meeresbiologischen Kurs in List und auf Helgoland für Studenten und Lehrer. Als langjährige Gesamtleiter der BAH-Kurse fungierten in List Dr. Erich Ziegelmeier, Dr. Gerhard Drebes, Dr. Hermann Kayser und auf Helgoland Dr. Klaus Lüning sowie Dr. Klaus Anger. Die technische Betreuung von Gastforschern und Kursen leitete auf Helgoland Günther Tad- 


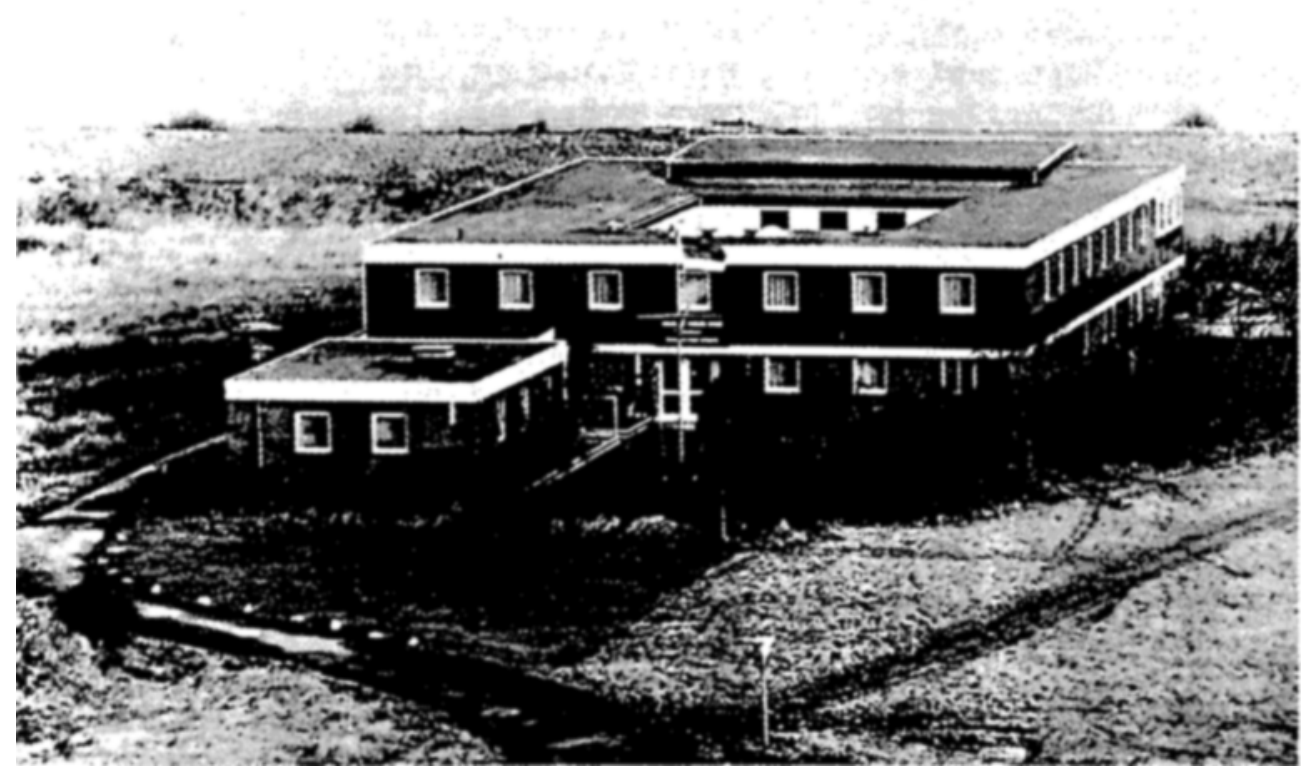

Abb. 30. Das "Wilhelm-Mielck-Haus" auf Helgoland, erbaut 1968. Photo: Archiv der BAH.

day (Abb. 28) mit langjährigen Mitarbeitern wie Brigitte Rauch. In List war Manfred Sturm für Kurse und Materialversand zuständig.

Die BAH bot und bietet auch Spezialkurse an, etwa über Protisten, Bakterien oder Crustaceen. Die Möglichkeiten Helgolands und der BAH für Lehre und Forschung wurden auch durch Prof. Kinne und seine Mitarbeiter intensiv genutzt. So fanden im Jahr 1964 gleich drei Kurse unter Prof. Kinnes Leitung hintereinander statt, ein Einführungskurs (4.-15. 8.), dessen Programm in Tabelle 6 im Anhang zu finden ist, ein Meeresbiologisches Praktikum für fortgeschrittene Studierende (17.-22. 8.) und ein zweiter Allgemeiner Kurs $(25.8,-4.9$.$) .$

Studenten sind ein belebendes Element in der BAH. Prof. Kinne berichtete darüber in der obenerwähnten Ansprache im Jahr 1992: „Nachteilig für die BAH als Bundesforschungsanstalt war das Fehlen des belebenden Elementes der Jugend. Diplomanden und Doktoranden stellen oft unbequeme Fragen und Forderungen. Das hält auf Trab und fördert die Beweglichkeit. Und diese jungen Mitarbeiter erbringen hohe Arbeitsleistungen. Das erweitert und intensiviert das Forschungspotential. Davon profitieren die Universitäten mehr als gemeinhin bekannt ist - oder zugegeben wird. In den sechziger Jahren war ich der einzige in der BAH, der habilitiert war, und zwar an der Universität Kiel. So begann ich, Kieler Studenten als Doktoranden auf Helgoland anzusiedeln. “ In vielen Fällen gibt es einen engen Zusammenhang zwischen der Teilnahme an Kursen in der BAH auf Helgoland oder in List, einer Rückkehr als studentische Hilfskraft, der Arbeit an einem Examensthema und der weiteren Verbindung als Gastforscher oder sogar als Mitarbeiter im Institut. 


\section{Die BAH 1972: Abteilungen, interdisziplinäre Forschung, Kollegium und Wissenschaftlicher Beirat}

Der Jahresbericht 1971 hat ein Vorwort des Bundesministers für Bildung und Wissenschaft, Dr. Klaus von Dohnanyi, in dessen Zuständigkeit die BAH am 1. 1. 1971 überging. Durch die Trennung vom Ministerium für Ernährung, Landwirtschaft und Forsten und von der Bundesforschungsanstalt für Fischerei wurden eine größere Selbständigkeit und ein engerer Bezug zur Grundlagenforschung hergestellt und die Bildungsaufgabe betont.

Unter dem neuen Minister wurde mit Wirkung vom 1.5.1972 die Arbeit an der BAH neu organisiert: Ichthyologie, Physiologie und Strahlenbiologie wurden als Abteilungen aufgegeben und dafür die Abteilung Experimentelle Ökologie neu eingerichtet; die Abteilung Planktologie wurde fortan "Biologische Ozeanographie“ genannt. Tabelle 2 enthält diese neue Gliederung und die Mitarbeiter der BAH, Abb. 31 zeigt das Organisationsschema. Mit Wirkung vom 15.12. 1972 wurde die BAH schließlich dem Geschäftsbereich des Bundesministers für Forschung und Technologie zugeordnet.

Der Leitende Direktor der BAH, Prof. Kinne, war im Nebenamt Professor an der Universität Kiel, führte als solcher Kurse für fortgeschrittene Studierende an der BAH durch und gab Doktoranden Anleitungen für ihre Arbeiten. Zugleich sorgte er mit dem mehrbändigen Werk "Marine Ecology" als Gründer, Herausgeber und Verfasser mehrerer Kapitel für die Grundlage der ökologischen Arbeiten an der BAH. In weltweiter Zusammenarbeit namhafter Fachgelehrter entstand die erste umfassende Darstellung unseres Wissens über mikrobielles, pflanzliches und tierisches Leben im Meer und in küstennahen Gewässern

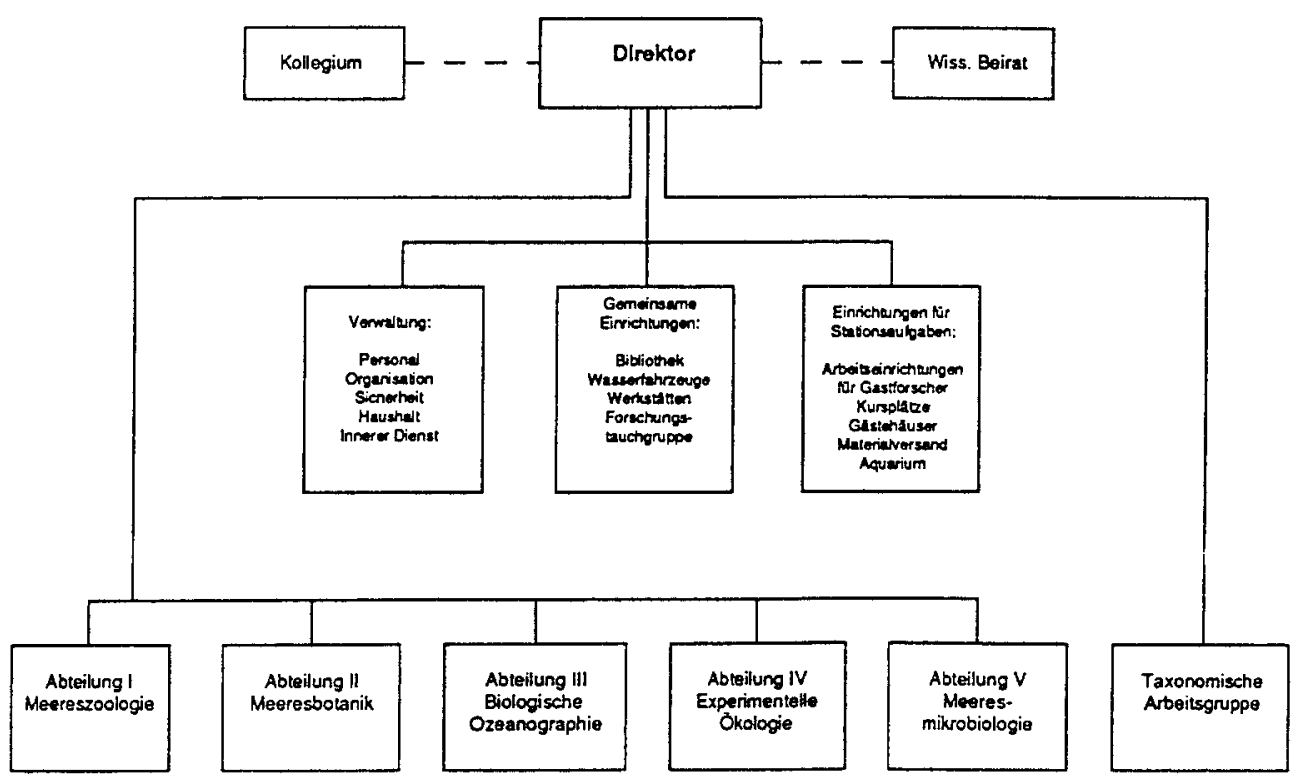

Abb. 31. Organisationsplan der BAH (Jahresbericht 1989). 
Verwaltungsleiter der BAH war ab 1971 Uwe Kersten. Seine Vorgänger waren bis 1964 Erich Windt, ab 1965 Heinrich Strehlow, ab 1966 Wilhelm Vollmer und ab 1967 Peter Bradhering. Frau Agnes Heinemann blieb von 1952 bis 1981 eine zentrale Verwaltungskraft; als Beispiele für weitere langjährige bewährte Mitarbeiterinnen der Verwaltung seien hier genannt: Frau Marie-Luise Bertram im Bereich der Gastforscher- und Kursbetreuung (seit 1968) und Frau Ursel Heitmann (seit 1981).

Die Leitung der Abteilung Meereszoologie hatte Prof. Kinne auf Dr. Hans-Peter Bulnheim übertragen, der sich mit Atmungsphysiologie und Geschlechtsbestimmung euryhaliner Gammariden beschäftigte. Dr. Dietrich Siebers arbeitete über Mechanismen der Osmoregulation bei der Strandkrabbe Carcinus maenas, Dr. Tesch über die Biologie des Aals, mit Fahrten in deutsch-niederländischer Zusammenarbeit zur Untersuchung des Aallarvenbestandes im Ostatlantik und mit Versuchen zur Wanderung und Orientierung von Aalen, die mit Ultraschallsendern markiert waren. Das Buch "Der Aal“ erschien 1973 in 1. Auflage und in der Folge auch als englische Übersetzung und als 2. deutsche Auflage (Tesch, 1973, 1977, 1983). Dr. Werner führte in Hamburg seine Untersuchungen über Lebensgeschichte, Systematik und Evolution der Nesseltiere weiter und konnte erstmals den vollständigen Lebenszyklus einer Cubomeduse aufklären. Dr. Ziegelmeier untersuchte seit 1949 Artenzusammensetzung und Bestandsdichte des Makrobenthos im Ostteil der Deutschen Bucht. Dabei war der strenge Winter 1962/63 von entscheidender Bedeutung für die Fluktuationen der Bestände. Eine interessante Filmdokumentation betraf den Nahrungserwerb von Schlangensternen.

In der Abteilung Meeresbotanik arbeitete der Abteilungsleiter Dr. Kornmann weiter auf Helgoland über Entwicklung und Taxonomie der Benthosalgen (Details bei Mollenhauer \& Lüning, 1988) und bereitete mit seinem Mitarbeiter Paul-Heinz Sahling eine zusammenfassende Darstellung der Helgoländer Benthosalgen vor (Kornmann und Sahling, 1977). Auch nach seiner Emeritierung 1972 bildete er bis zu seinem Tode 1993 mit P. H. Sahling eine unermüdliche und sehr erfolgreiche Arbeitsgruppe an der BAH. Dr. Lüning, seit 1974 mit der Leitung der Abteilung beauftragt, untersuchte auf Helgoland den Stofftransport bei Laminaria-Arten und begann mit Experimenten über das Licht als Steuerungsfaktor bei der Algenentwicklung. Dr. Drebes wechselte 1972 von Helgoland nach List, begann dort mit Bestandsaufnahme und Kultivierung des Phytoplanktons für taxonomisch-ontogenetische Untersuchungen, und 1974 erschien sein Einführungsbuch in die Taxonomie und Entwicklung des marinen Phytoplanktons (Drebes, 1974). Dr. Kesseler setzte in List seine zellphysiologischen Untersuchungen an Planktondiatomeen fort.

Die Abteilung Biologische Ozeanographie wurde bis zur Berufung von Prof. Gillbricht im Jahr 1973 von Prof. Kotthaus in seinem letzten Dienstjahr geleitet. Prof. Kotthaus arbeitete in der Zentrale Hamburg an der Taxonomie der Fische des Indischen Ozeans anhand von Material der "Meteor"-Expedition 1964/65. Dr. Hagmeier untersuchte Hydrographie, Chemie und Phytoplanktongehalt des Wassers bei Helgoland und führte die "Kabeltonnendaten " fort, ergänzt durch Wasserproben aus der Deutschen Bucht. Die technischen Mitarbeiter Klaus Treutner und Peter Mangelsdorf entwickelten die chemische Methodik weiter. Dr. Hickel wertete die von den Feuerschiffen der südlichen Deutschen Bucht genommenen Planktonproben aus und begann mit Dr. Drebes und Dr. Gustav-Adolf Paffenhöfer eine Studie der Ökologie des Nordsylter Wattenmeeres. Dr. Paffenhöfer gelang die kontinuierliche Kultur von planktischen Copepoden und erstmals die Kultur der Appendicularie Oikopleura dioica. 
Dr. Uhlig, der Leiter der Abteilung Experimentelle Ökologie, setzte auf Helgoland die langfristigen, experimentell-ökologischen Untersuchungen an Noctiluca auf See und im Labor fort und entwickelte Methoden zur Erfassung der marinen Bodenmikrofauna. Dr. Wulf Greve untersuchte Zooplankton im Auftriebsgebiet der westafrikanischen Küste und testete auf der "Meteor" verschiedene, von ihm entworfene Hälterungssysteme. Dr. Manfred Hoppenheit untersuchte in Hamburg das Verhalten von Populationen des Copepoden Tisbe holothuriae bei verschieden hohen wöchentlichen Entnahmeraten. Dr. Harald Rosenthal führte strahlenbiologische Untersuchungen an Keimzellen und Embryonen des Herings aus.

In der Abteilung Meeresmikrobiologie untersuchte der Leiter Dr. Gunkel Bakteriengehalt, Sauerstoffzehrung und Oberflächenspannung des Helgoland-Reede-Wassers. Zusammen mit Dr. Horst Altmann wurden die langjährigen Arbeiten über den mikrobiellen Abbau von Mineralölen weitergeführt. Dr. Wolfgang Krumbein erfaßte die Bakterienzahlen an der Grenzfläche Sediment-Wasser und im Sediment in der Helgoländer Bucht, der weiteren Nordsee und der Biskaya. Er führte sediment-mikrobiologische Untersuchungen im Golf von Aqaba aus, und auch die Geologie Helgolands über und unter Wasser wurde von ihm zusammen mit Gastforschern untersucht (Krumbein \& van der Pers, 1974; Krumbein, 1975). Diese Arbeiten wurden später bei Diskussionen um Naturschutz und Küstenschutz an der Nordostseite der Insel wichtig. Dr. Karlheinz Moebus arbeitete über die antibakterielle Aktivität des Meerwassers und ging 1972 für ein Jahr als Stipendiat zum Mikrobiologen Prof. Sieburth in die USA.

Die Interdisziplinäre Arbeitsgruppe zur Erkennung, Bekämpfung und Verhütung der Meeresverschmutzung untersuchte Auswirkungen einer Entsorgung von Rotschlamm in die Nordsee, der bei der Gewinnung von Aluminium aus Bauxit anfällt. Bei einer Probeverklappung im Jahr 1971 hatte sich gezeigt, daß der feine Schlamm auch bei geringen Strömungen über große Flächen ausgebreitet wird. Wegen der dadurch entstehenden Schäden an den Bodentieren wurde von einer Verbringung in die See abgesehen. Bereits im Jahr 1967 waren BAH-Mitarbeiter mit verschiedenen umweltbezogenen Arbeiten an die Öffentlichkeit getreten. Dr. Gunkel sprach über seine experimentellen Untersuchungen über ölabbauende Bakterien im Meer bereits auf dem Kolloquium der Deutschen Forschungsgemeinschaft "Abwassereinflüsse in Küstennähe" am 18.3.1967 in Bremerhaven. Im ersten Heft des ersten Bandes von Marine Biology veröffentlichen Prof. Kinne und Dr. Rosenthal ihre Versuche über die Wirkung schwefelsäure- und eisenhaltiger Abwässer auf Befruchtung, Embryonalentwicklung und Larven des Herings, veranlaßt durch die beabsichtigte Verklappung von Abfallprodukten aus der Titangewinnung (Kinne \& Rosenthal, 1967). Dr. Hermann Kayser testete auf Helgoland den Einfluß verschiedener Konzentrationen von Rotschlamm, Quecksilber, Zink und Cadmium auf die Vermehrung von Flagellaten. Außerdem untersuchte er die Anreicherung von Cadmium in Planktonalgen und in Tunikaten, Bryozoen, Kamptozoen und Folliculiniden, die mit Cadmium-kontaminierten Planktonalgen gefüttert waren. Dr. Kayser übersiedelte 1975 von Helgoland nach List.

Dr. Hoppenheit in Hamburg fungierte als Ansprechpartner der BAH in Fragen der Meeresverschmutzung, der damit verbundenen Gesetzesfragen, nahm an vielen einschlägigen Sitzungen teil und bearbeitete für die BAH den entsprechenden Schriftverkehr. Dr. Klaus-Richard Sperling begann in Hamburg im Jahr 1972 seine Tätigkeit mit der Entwicklung von Bestimmungsmethoden für giftige Schwermetallionen und sam- 
melte erste Erfahrungen mit Cadmium und Zink. Zusammen mit Dr. Rosenthal arbeitete er über die konzentrationsabhängige Aufnahme von Schwermetallionen durch Fischembryonen und mit Dr. James Markham sowie Dr. Lüning über die Wirkung von Schwermetallionen auf das Wachstum von Benthosalgen. Dr. Hoppenheit, Dr. Karl Klöckner, Dr. Siebers, Dr. Sperling sowie Dr. Hein v. Westernhagen beobachteten Überleben, Stoffwechsel und Populationsdynamik von Garnelen, Polychaeten und Tisbe unter dem Einfluß von Schwermetallen sowie die Aufnahme von Transuranen durch Amphipoden. Viele Untersuchungen von Dr. Rosenthal und Dr. Hein v. Westernhagen befaßten sich mit der Wirkung von Abwasser-Inhaltsstoffen auf die Entwicklung unserer Nutzfische Hering, Hornhecht, Scholle, Flunder und Kliesche, wobei auch der Einfluß der PCB-Belastung (polychlorierte Biphenyle) der Elterntiere auf die Überlebensrate der Nachkommen geprüft wurde (Dr. Rosenthal, Dr. Sperling, Dr. Hein v. Westernhagen, später auch Patricia Cameron). In Zusammenarbeit mit Dr. Volkert Dethlefsen (BFAFi) wurde die Wirkung von PCBs auf die Enzymaktivität des Kabeljaus geprüft (Dr. Hansen). Dr. Klöckner und später Thomas Kaufmann führten analoge Versuche mit Seeigeln, Austern, Polychaeten und Ascidien durch.

Für Untersuchungen der Arbeitsgruppe Meeresverschmutzung nutzt die BAH zusammen mit dem Deutschen Hydrographischen Institut (seit dem 1.7. 1990 Bundesamt für Seeschiffahrt und Hydrographie) und der Bundesforschungsanstalt für Fischerei das Isotopenlaboratorium in Hamburg-Sülldorf. Prof. Bückmann hatte schon eine Abteilung "Strahlungsbiologie" an der BAH eingerichtet, deren Leiter erst Dr. Aurich und später Dr. Hoppenheit wurden: dieser war dann maßgeblich an Planung und Arbeiten im Isotopenlabor beteiligt. Auf dem BAH-Symposium 1967 wurde über das in Bau befindliche Labor gesprochen, Ende 1969 wurde es fertig. Es geht darin um die Untersuchung der Radioaktivität im Meer und seinen Organismen und besonders um die aus dem Meer gewonnene Nahrung für den Menschen: Durch die Nutzbarmachung der Kernenergie besteht die Gefahr der Verbreitung von radioaktiven Abfällen. Kontrollmessungen in Wasserproben, Pflanzen, Tieren und Sedimenten sind genau so nötig wie Laborversuche über die Aufnahme, Weitergabe und Schädlichkeit verschiedener durch den Menschen eingetragener Stoffe. Neben den Untersuchungen mit Radioisotopen werden in Sülldorf in zunehmendem Maße spurenanalytische Verfahren entwickelt und angewandt (Nies et al., 1994).

Die Interdisziplinäre Arbeitsgruppe zur Forschung über Grundlagen der marinen Aquakultur verfolgte die schwierige Aufgabe der Brut- und Besatzerzeugung von Nutzfischen. Dr. Jürgen Flüchter hatte zu diesem Thema bereits seit 1962 im Hafenlabor auf Helgoland wertvolle Erfahrungen gesammelt. In Bewältigung technischer Probleme entwickelte er einen wirksamen und später von Dr. Wulf Greve in seinem „Planktonkreisel” gebrauchten Innenfilter (Greve, 1968). Dr. Flüchter bearbeitete auch den Plan "für den Bau eines zur Zucht von Meeresorganismen und für experimentell-ökologische Untersuchungen geeigneten Laboratoriums" (Jahresbericht 1972). Im Jahr 1972 wurden von Dr. Flüchter auf Helgoland gezüchtete junge Seezungen von 15-20 mm Länge nach Hamburg überführt und dort von Dr. Rosenthal in einem geschlossenen Meerwasserkreislauf mit biologischer Aufbereitung und Bypass-Ozonisierung erfolgreich weiter aufgezogen. In diese interdisziplinäre Arbeitsgruppe gehörten auch die fischpathologischen Untersuchungen von Dr. Gerhard Lauckner in List.

Mit der 1972 erfolgten Einrichtung einer Abteilung für experimentelle Ökologie 
wurde ein Anliegen verwirklicht, das schon Prof. Arthur Hagmeier verfolgt hatte. In seinem Schreiben an den Reichsminister für Wissenschaft, Erziehung und Volksbildung am 18. 7. 1941 "Betr.: 50jähriges Bestehen der Biologischen Anstalt Helgoland im Jahre 1942 und Anmeldung zum preußischen Haushaltsplan für 1942" hieß es: "Bei den Besprechungen 1934 über den äußeren Aufbau der Anstalt wurde mir zugesagt, daß 1942, im Jubiläumsjahr, eine Neuordnung des Personaletats stattfinden könnte... Die wichtigsten neu einzurichtenden Planstellen sind: 1. Die Stelle eines Kustos für experimentelle Ökologie ... Auf die verschiedenen Aufgaben der Biologischen Anstalt Helgoland und auf die Notwendigkeit, diese Arbeiten gerade auf Helgoland auszuführen, sei nochmals kurz hingewiesen. Bei den Forschungsaufgaben handelt es sich zur Hauptsache um ökologische Forschungen. Diese sollen eine zusammenfassende Übersicht schaffen über die Gesetzmäßigkeiten im Lebensraum und die damit eng verknüpften Erscheinungen im Lebenskreislauf und in der Bestandsveränderung der Organismen, namentlich auch der nutzbaren Meerestiere und Meerespflanzen. Bei den ökologischen Forschungen handelt es sich nicht um einmalige Feststellungen, sondern um fortlaufende terminmäßige Untersuchungen und Experimente. ... Die Beobachtungen in der Natur müssen durch Experimente in Aquariums- und Züchtungsanlagen ergänzt werden ... ." Zusätzlich zur Kustodenstelle forderte Prof. Hagmeier "zwei Stellen für technische Inspektoren im Innen- und Außendienst, und zwar je eine für die Zoologie und für die Botanik als Aufrückungsstellen für männliche technische Assistenten", sowie die Umwandlungen vorhandener Planstellen, damit die "wirtschaftliche Lage auch bei den Helgoländer Verhältnissen gesichert ist". Schon Prof. Heincke kannte das Problem, bei teurer Lebenshaltung auf einer Insel Mitarbeiter zu gewinnen und zu halten. Das ist bis heute so geblieben.

Mit dem Organisationserlaß über die BAH vom 11.1.1971 wurden auch Aufgaben und Rechte des Kollegiums festgelegt, eines neuen Gremiums zur Mitwirkung bei der Gestaltung des Arbeitsprogramms der BAH. Das Kollegium besteht aus dem Direktor, den Abteilungsleitern sowie je zwei aus dem Kreise der Wissenschaftler und Techniker gewählten Mitgliedern.

Ein zweites Gremium zur Beratung und Unterstützung der wissenschaftlichen Arbeit an der BAH ist der Wissenschaftliche Beirat. Schon während der Trägerschaft der BAH durch das Bundesministerium für Ernährung, Landwirtschaft und Forsten (BML) existierte ein Wissenschaftlicher Beirat (vgl. Kapitel 10). Für 1968 werden auf dem Deckblatt der Helgoländer wissenschaftlichen Meeresuntersuchungen, Band 17, folgende $10 \mathrm{Mit}$ glieder genannt: Prof. Wolfgang Bargmann (Kiel), Vorsitzender, Prof. Peter Ax (Göttingen), Prof. R. W. Kaplan (Frankfurt/Main), Prof. Kurt Lillelund (Hamburg), Prof. Rolf Siewing (Erlangen-Nürnberg) und je ein Vertreter der Freien und Hansestadt Hamburg, der Freien und Hansestadt Bremen, des Ministers für Ernährung, Landwirtschaft und Forsten des Landes Schleswig-Holstein, des Ministers für Ernährung, Landwirtschaft und Forsten des Landes Niedersachsen und der Ständigen Konferenz der Kultusminister der Länder. Der Wissenschaftliche Beirat im Auftrag des Bundesministers für Forschung und Technologie (BMFT) konstituierte sich am 5. 5. 1982 neu mit folgenden in- und ausländischen Wissenschaftlern, die vom BMFT für die Dauer von 5 Jahren als Mitglieder berufen wurden: Prof. Peter Ax (Göttingen), Prof. Wolfgang Ernst (Bremerhaven), Prof. Bengt-Owe Jansson (Askö, Schweden), Prof. Wilhelm Nultsch (Marburg, stellvertretender Vorsitzender), Prof. Jürgen Overbeck (Plön), Prof. H. Postma (Texel, Niederlande; Vorsitzender) und Prof. Bernt Zeitzschel (Kiel). Themen der Sitzungen des Beirats waren u. a. die Be- 
teiligung der $\mathrm{BAH}$ in der marinen Umweltschutzforschung, die wissenschaftliche Nutzung des Ökolabors auf Helgoland, der Naturschutz im Sylter Raum, die rechnergestützte Ökosystemanalyse, die Neuordnung der Meeresforschung im Hamburger Raum, die Wiederbesetzung der Direktorenstelle und die Zukunft der BAH.

\section{Taxonomische Arbeitsgruppe}

Am 1. 1. 1972 übernahm die BAH als Rechtsträger die "Taxonomische Arbeitsgruppe zur Bearbeitung des biologischen Materials aus Expeditionen deutscher Forschungsschiffe". Die Taxonomische Arbeitsgruppe (TAG) wurde 1969 auf Anregung von Prof. Sebastian Gerlach gegründet und zunächst durch die DFG finanziert. Im Jahr 1972 bestand sie aus 10 Biologen, die an verschiedenen Instituten untergebracht waren. Dr. Hans Georg Andres arbeitete am Zoologischen Institut und Museum in Hamburg (ZIM) über die Amphipoden der Kaltwasserströme von Südafrika und Südamerika. Dr. Ilse Bartsch (ZIM) untersuchte neue Halacariden von der Großen Meteor-Bank, der Josephine-Bank und dem Eulitoral von Südafrika sowie Ophiuriden, die vor allem von der "Walther Herwig " aus dem Südatlantik stammten. K.-H. Becker arbeitete am Zoologischen Institut der Universität Kiel im Rahmen seiner Dissertation über Tiefsee-Harpacticiden aus dem Peru-Graben und Becken des Atlantik. Ingo Freudenhammer bearbeitete am Institut für Meeresforschung (IfM) Bremerhaven freilebende Tiefsee-Nematoden, die von der "Meteor" und der "Anton Bruun" (Peru-Graben) stammten. Dr. habil. Gesa Hartmann-Schröder (ZIM) untersuchte Polychaeten aus der Nordsee und dem Benguela-Strom-Gebiet, Helga Kapp (ZIM) Chaetognathen von der Indian-Ocean-Expedition der "Meteor" sowie aus dem Atlantik und, zusammen mit Prof. Bückmann, Appendikularien. Dr. Anton Lelek arbeitete an der BAH in Hamburg über die Fischbrut aus Fängen der "Friedrich Heincke" nach Einarbeitung durch Dr. Walter Nellen vom Institut für Meereskunde Kiel. Dr. Karin Riemann-Zürneck untersuchte im IfM Bremerhaven Aktinien von drei Südatlantik-Expeditionen der "Walther Herwig" sowie Vergleichsmaterial aus dem Nordatlantik. Dr. Hartmut Schinkowski arbeitete am IfM Kiel über Decapoden aus RingtrawlFängen aus dem Auftriebsgebiet vor NW-Afrika sowie über Cephalopoden, und Dr. Renate Weigmann untersuchte am IfM Kiel Euphausiden von der Großen Meteor-Bank und der Josephine-Bank.

Die Tätigkeit der Taxonomen umfaßt nicht nur vergleichende und beschreibende Studien an konserviertem Material, sondern auch die Teilnahme an Reisen, Auslandsaufenthalte sowie Beobachtungen am lebenden Objekt in situ und im Labor. Durch die genaue Bestimmung und Benennung der Organismen sowie die Erstellung von Bestimmungsliteratur liefern die Taxonomen wichtige Grundlagen für die Arbeiten der Ökologen.

Zu den genannten 10 Mitgliedern der Taxonomischen Arbeitsgruppe kamen im Jahr 1972 Dr. Knud Schulz (ZIM) für die Bearbeitung calanider Copepoden hinzu, 1973 Dr. Malte Elbrächter als Dinoflagellaten-Spezialist (BAH, List), 1974 Dr. Hans-Christian John zur Bestimmung von Fischlarven und 1977 Dr. Kunigunde Hülsemann (BAH, Hamburg) für calanide Copepoden. Auf diese Weise bildete sich im Laufe der Jahre ein Stamm von Taxonomen, verstärkt durch einzelne von der DFG befristet angestellte Mitarbeiter. Es wurde ein Koordinierungs-Ausschuß gebildet, dem im Jahr 1973 Prof. Sebastian Gerlach, Prof. Gotthilf Hempel, Prof. Adolf Kotthaus, Prof. Kurt Lillelund und Prof. Gerhard Hart- 


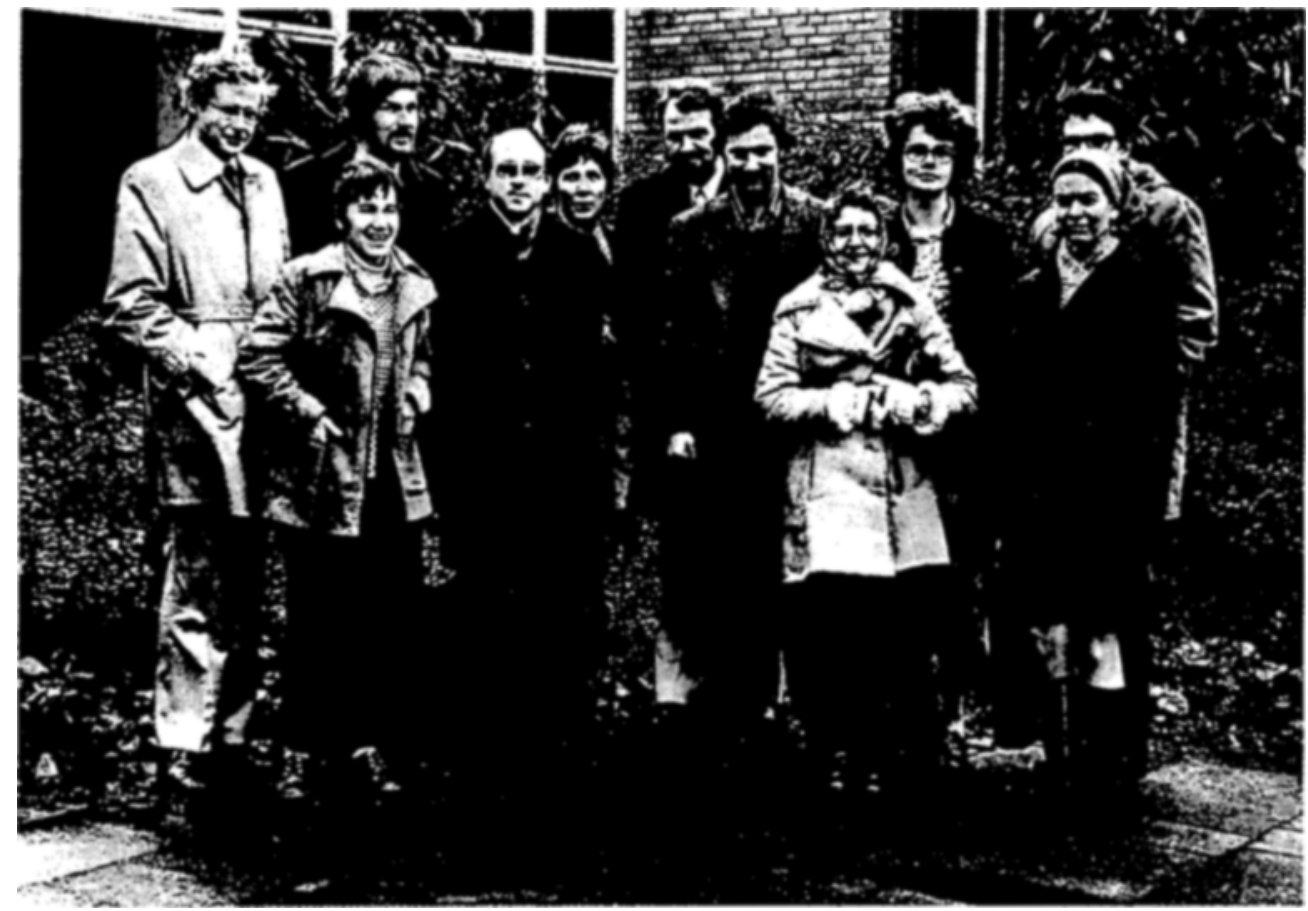

Abb. 32. Mitglieder der Taxonomischen Arbeitsgruppe 1974 vor der BAH-Zentrale in der Bundesforschungsanstalt für Fischerei. Von links nach rechts, hintere Reihe. Dr. K.-H. Becker, Dr. Knud Schulz. Dr. Renate Haass, Dr. Malte Elbrächter, Dr. Karin Riemann-Zürneck, Dr. Hans Georg Andres; vordere Reihe. Dr. Ilse Bartsch, Dr. Hans-Christian John, Dr. Hartmut Schinkowski، Dipl.-Biol.

Helga Kapp, Dr. Gesa Hartmann-Schröder. Photo: Archiv der BAH.

mann angehörten. Die Stellung des Leitenden Taxonomen wurde nacheinander von Prof. Kotthaus, Dr. Hartmann-Schröder, Dr. Hülsemann, Dr. Hartmann-Schröder und Dr. Andres eingenommen. Die regionalen Schwerpunkte der Untersuchungen werden durch die großen nationalen und internationalen Programme der deutschen Meeresforschung bestimmt. Sie liegen in jüngster Zeit speziell in den polaren Regionen, u. a. mit den Programmen European Polarstern Study (EPOS), Biological Investigations of Marine Antarctic Systems and Stocks und BIOMASS. Die Gruppe der Taxonomen im Jahr 1974 ist in $\mathrm{Abb} .32$ vorgestellt.

\section{Neubau in List auf Sylt}

Beim Amtsantritt von Prof. Kinne im Jahr 1962 wurde durch das Bundesministerium für Ernährung, Landwirtschaft und Forsten (BML) zunächst festgelegt, daß die Liegenschaft der BAH auf Sylt aufgelöst und das Sylter BAH-Personal nach Hamburg oder Helgoland versetzt werden sollte (Kinne, mdl. Mitteilung). Es gelang Prof. Kinne nur mit Schwierigkeiten, das BML von diesem Vorsatz abzubringen und die Litoralstation List zu erhalten und auszubauen. Im Jahr 1972 wurde ein neues Laborgebäude in List fertiggestellt (A.bb. 33). Die Bemühungen darum begannen im November 1964 mit einem Schreiben von Prof. Kinne an den Wissenschaftsrat, in dem zunächst von Instandsetzung und 


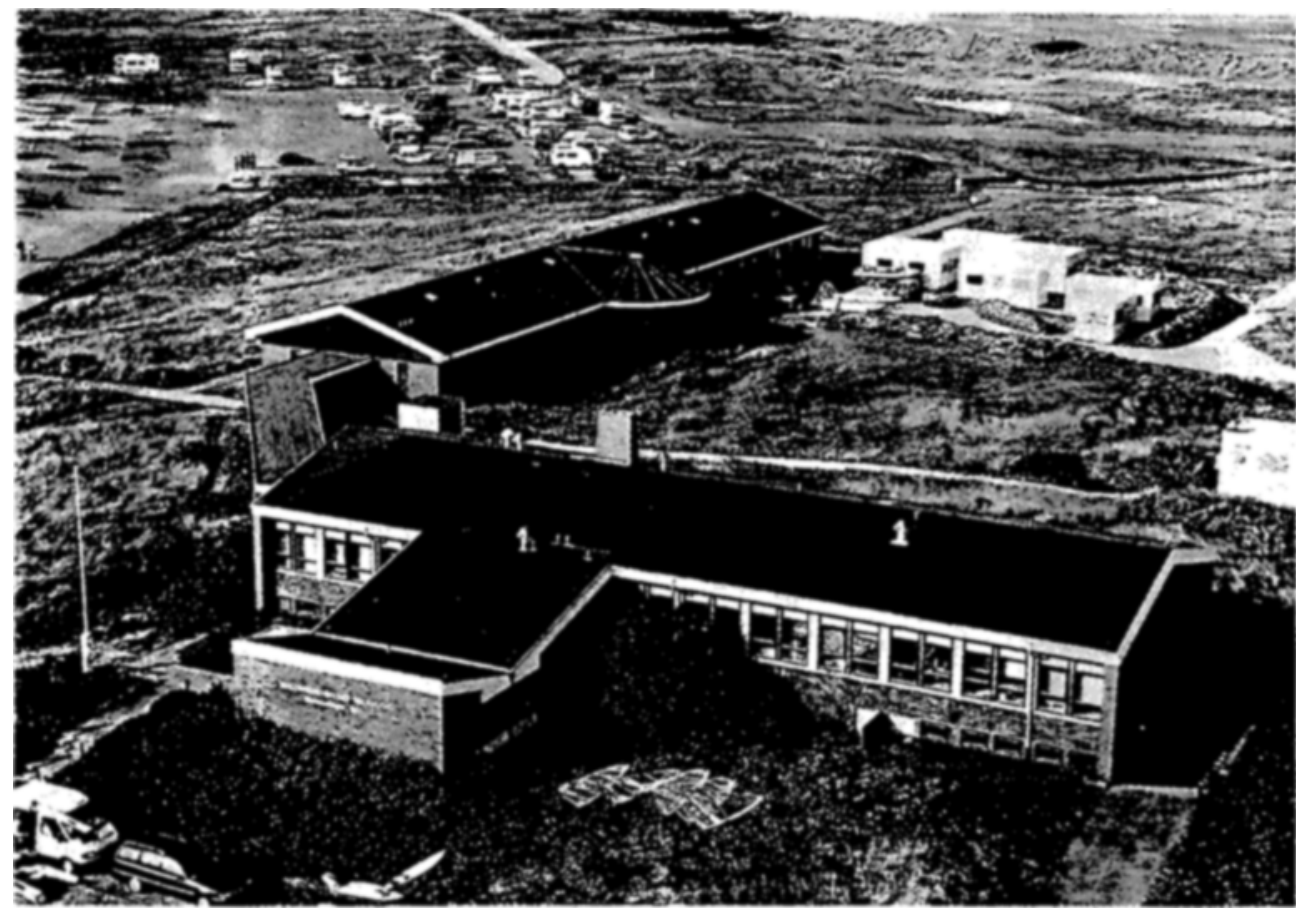

Abb. 33. BAH-Neubauten in List 1993. Im Vordergrund: Laborgebäude von 1972. Durch einen Gang verbunden, dahinter: Gastforscherlabor und Bibliothek, genutzt seit 1979. Photo: V. Frenzel.

Aufstockung des vorhandenen Labors und von einer Renovierung des Gästehauses die Rede war. Das Landesbauamt Flensburg wurde zur Beratung hinzugezogen. Es stellte sich heraus, daß eine Vergrößerung der alten "Bombenbäckerei" nicht zweckmäßig und relativ teuer wäre. Eine Beratung von Prof. Kinne im April 1966 mit Vertretern von Bundesschatzministerium, Bundesvermögensstelle, Verteidigungsministerium und Prof. Ax (Göttingen), einem Hauptnutzer der Gastforschermöglichkeiten in List, ergab, daß ein Neubau in der Nähe des Hotels "Mövenflug" an der Nordwestecke des Flugplatzgeländes große Vorteile hätte. Zunächst mußten jedoch die schwierigen Grundstücksvermögensverhältnisse geklärt werden.

Zwei Monate später kam es in der Station zu einem abendlichen Besuch des Schatzministers Dr. Dollinger, als Entscheidungsträger für die Vergabe des Baulandes. Dr. Aurich als Leiter der Station konnte einen Eindruck interessanter Arbeit in betriebsamer Enge vermitteln. Die Studenten des Kurses von Prof. Luther aus Darmstadt kamen gerade mit Pflanzen und Tieren aus dem Watt zurück, und auch die Doktoranden aus Göttingen waren mit ihren Meiofauna-Proben beschäftigt. Die planmäßige Belegschaft der BAH war gegenüber der Gästezahl recht klein und bestand aus Dr. Aurich, Dr. Kesseler, Dr. Ziegelmeier und 7 Mitarbeitern für Labortechnik sowie für den Betrieb von Schiffen und Gebäuden.

Ende 1966 wurde vom Ernährungsminister der Stellen- und Raumbedarfsplan für die Wattenmeerstation genehmigt. Unter Beibehaltung des vorhandenen Altbaus sollte der 


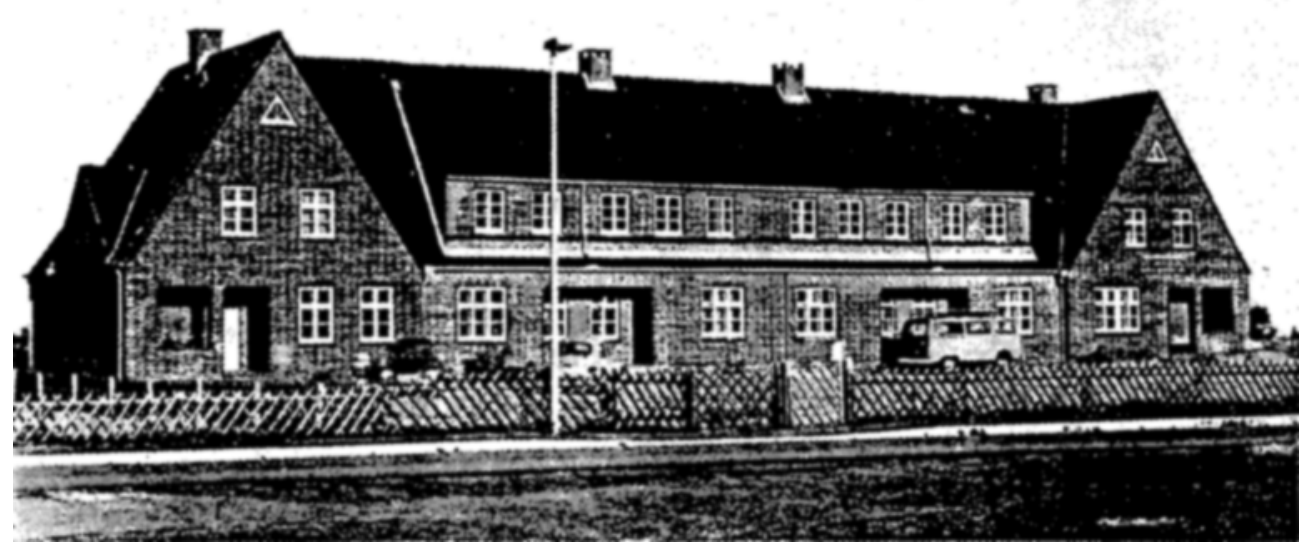

Abb. 34. "Adolf-Bückmann-Haus" (ehemaliges „Strohdachhaus") in List 1976. Photo: Manfred Sturm.

Neubau so geplant werden, daß eine Erweiterung möglich war. Im Jahr 1967 konnte das Baugrundstück gesichert werden, nachdem die Bundeswehr auf die militärische Nutzung verzichtet hatte. Der Vorentwurf des Landesbauamtes Flensburg, nach einer Vorgabe von Dr. Kesseler, wurde vom Bundesschatzminister genehmigt, die Kosten wurden auf 964000 DM festgesetzt und in den Entwurf des Bundeshaushalts für 1968 aufgenommen.

Neben der genauen Planung des Laborneubaus liefen seit 1968 die Bemühungen um bessere Räume für Kurse und Gäste. Die Beiratssitzungen im Februar und Juni 1968 befaßten sich damit. Außerdem fanden Besprechungen in List unter Beteiligung der Bauabteilung der Oberfinanzdirektion Kiel statt. Dabei wurde ein flaches Gebäude der ehemaligen Wehrmacht، später Gaststätte „Mövenflug”, für Umbau und Nutzung vorgesehen. Die daneben stehenden Wohnhäuser waren jedoch nicht mehr zu gebrauchen und wurden später abgerissen. Die Stiftung Volkswagenwerk wurde um die Finanzierung gebeten. Es kam zwar zu einem alle Aufgaben der BAH umfassenden Entwurf, aber zunächst zu keiner Lösung für die Bezahlung von Ausführung und Folgekosten. Am 1.3. 1969 erfolgte die Ausschreibung der Arbeiten für den Neubau des Laborgebäudes, das bis zur Fertigstellung noch viel Zeit brauchen und dem besonders engagierten Dr. Kesseler viel Mühe machen sollte. Auch die Versorgung mit Meerwasser aus dem an Trübstoffen reichen Wattenmeer gehörte zu den schwierigen, aber schließlich gut gelösten Problemen (Kesseler, 1981).

Die Festrede zur Einweihung des Neubaus in List am 16. Juni 1972 hielt Prof. Ankel, der als früherer Mitarbeiter und Gastforscher der Lister Station eng verbunden war und die Veränderungen im Wattenmeer über 50 Jahre erlebt hatte. Ein ohne den Menschen entstandenes Existenzgefüge, so sagte er, wurde hier durch den Menschen zerstört. Was 
in kleinem Umfang geschehen wäre, drohe jetzt dem Lebendigen auf der ganzen Welt. Prof. Ankel sprach weiter über den Unterschied zwischen Wissen und Verstehen. Es brauche viel Zeit, um von einem Organismus die Antwort zu erhalten, die einem weiterhilft, den Organismus zu verstehen. Hier in List gäbe es die Möglichkeit zu solcher Forschung und Einsicht, abseits von den „überfüllten Belehrungsmaschinen", zu denen die Universitäten geworden wären. Erst am 20.12.1979 konnte schlieBlich das ehemalige Restaurant "Mövenflug “ als Gastforscherlabor und Bibliotheksgebäude der BAH übergeben werden, nach einer Bauzeit von zweieinhalb Jahren für Umgestaltung und Verbindung mit dem 1972 eingeweihten Neubau. Das ehemalige "Strohdachhaus" konnte in voller Länge übernommen und renoviert werden und dient seitdem Gastforschern und Kursen als Unterkunft (Abb. 34).

\section{Das Ökolabor auf Helgoland}

Die Errichtung eines experimentell-ökologischen Labors mit modernen Einrichtungen für die Züchtung und Leistungsanalyse von Meeresorganismen war eine der Voraussetzungen für die Verwirklichung des Schwerpunktprogramms der Biologischen Anstalt. Die Bemühungen darum reichen bis 1963 zurück. Prof. Kinne schrieb im Jahresbericht für 1966: "Die Zucht mariner Tiere, Pflanzen und Mikroorganismen unter kontrollierbaren Umweltbedingungen (insbesondere Lichtklima, Temperatur, Salzgehalt, Wasserbewegung und hydrostatischer Wasserdruck) ist heute zum Schlüssel für das Verständnis der Dynamik des Lebens im Meer geworden. Neben den USA und der UdSSR schicken sich gegenwärtig auch Großbritannien, Frankreich, Holland, Japan und viele andere Kulturnationen an, moderne Züchtungsanlagen für Meeresorganismen in Betrieb zu nehmen. Es gilt, mit Hilfe umfangreicher technischer Einrichtungen eine maximale Manipulierbarkeit der wichtigsten Umweltfaktoren zu erreichen und genügend Gewächshausräumlichkeiten für eine Massenzucht der wichtigsten planktischen Futtertiere und -pflanzen zu schaffen. Innerhalb der Bundesrepublik bietet die Lage der Insel Helgoland die günstigsten Voraussetzungen für den Betrieb eines Züchtungslaboratoriums für Meeresorganismen. Ein geeignetes Grundstück, auf welchem auch die erforderlichen Außenanlagen (Versuchsteiche, Gräben, Becken) Platz finden können, ist vorhanden. Angesichts der gegenwärtigen angespannten Haushaltslage des Bundes wurde der ursprüngliche Raumbedarfsplan für das experimentell-ökologische Laboratorium seitens der Biologischen Anstalt Helgoland drastisch gekürzt. Es steht zu hoffen, daß der erneut vorgelegte, reduzierte Raumbedarf nunmehr anerkannt wird und im Haushaltsjahr 1969 mit den Bauarbeiten begonnen werden kann."

Nach einer gründlichen Planung des Laborteils mit verbundenem Bibliotheksteil wurde der Entwurf im Jahresbericht für 1970 vorgestellt. Gleichzeitig wurde berichtet, daß im März 1970 mit der vorgeschriebenen Tiefenräumung des Baugeländes angefangen werden konnte. Im Jahr 1971 wurden die Tiefenräumung abgeschlossen und die Ausschachtungsarbeiten begonnen. Dabei mußte nach einem Meerwassereinbruch im Bereich der Tief- und Filterbehälter das Grundwasser abgesenkt werden. Abbildung 35 zeigt die Ausdehnung der jetzt unter der Erde verborgenen Bauteile. Ende 1973 waren Laborgebäude und Züchtungshalle im Rohbau fertiggestellt. Jacob K. Holtmann, damals technischer Assistent von Prof. Kinne, erwarb sich große Verdienste bei der Detailplanung der Anlagen und bei der Bauaufsicht. Am 14. Mai 1974 fand das Richtfest für La- 


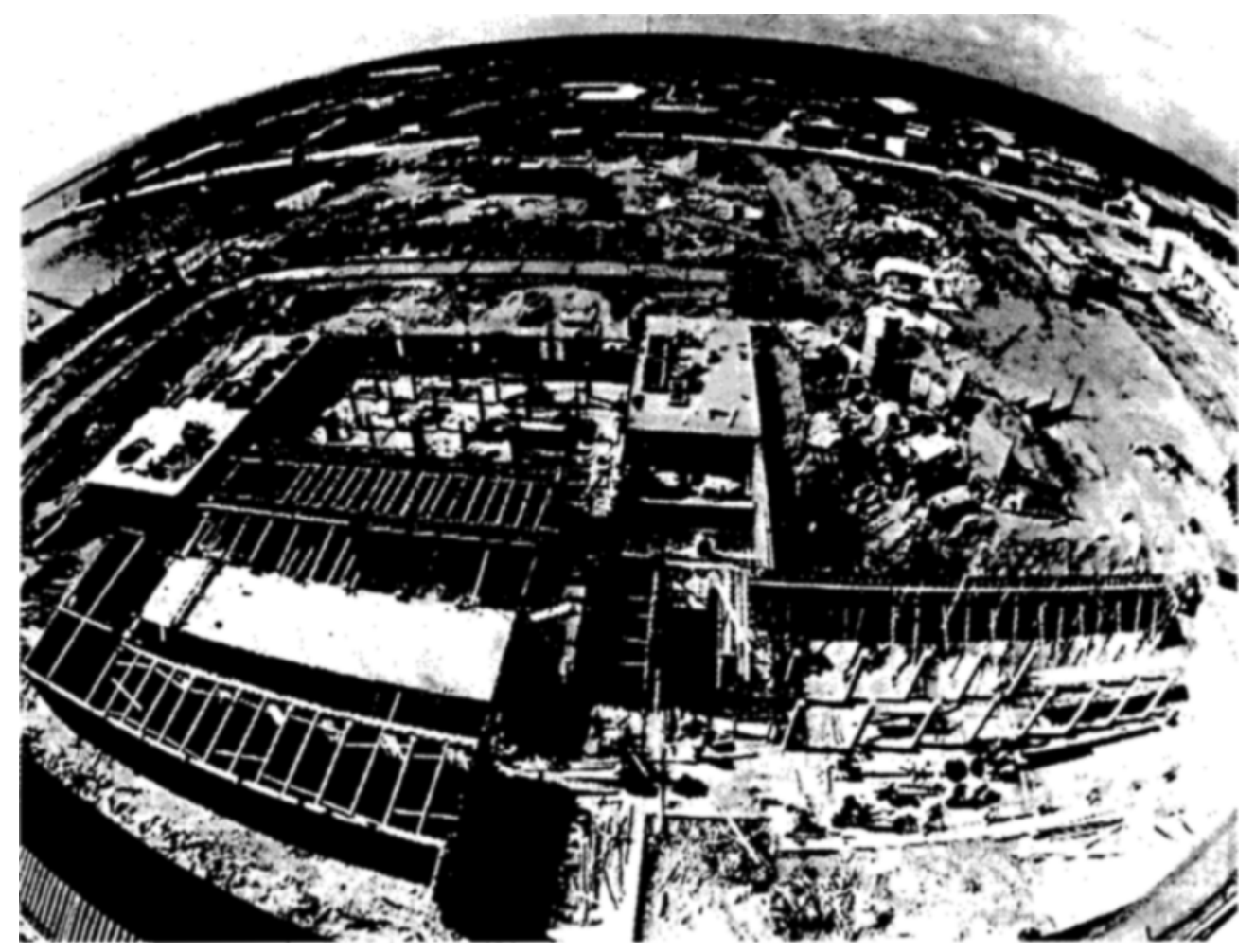

Abb. 35. Das Ökolabor der BAH auf Helgoland im Bau (1973). Photo: O. Cohrs. Aufnahme vom Baukran aus durch ein Fischaugen-Objektiv.

bor- und Bibliotheksgebäude statt. Trotz schlechter Wetterlage im Herbst wurden die Dach- und Verglasungsarbeiten so weit fertiggestellt, daß mit dem inneren Ausbau begonnen werden konnte. Die Installation des umfangreichen Röhrensystems für die verschiedenen Kreisläufe, die von den Tiefbehältern über die Kammern des Hochbehälters zur Züchtungshalle und zu den Zapfstellen der Freiterrasse führen, wurde 1975 abgeschlossen, ebenso die Elektroinstallation einschließlich der Montage der zahlreichen Meerwasserpumpen, Kühlaggregate, Luftkompressoren und des Lüftungssystems.

In Anwesenheit eines Vertreters des Bundesministers für Forschung und Technologie wurden am 8. 6. 1976 nach vierjähriger Bauzeit das Experimentell-ökologische Laboratorium und die Bibliothek an die BAH übergeben (Abb. 36). Die Baukosten in Höhe von rund 13 Mio. DM stellte der BMFT zur Verfügung. Außer dem Landesbauamt Itzehoe hatte das Architektenbüro Marlow und Partner an der Fertigstellung mitgewirkt. Eine Beschreibung des Hauses findet sich im Jahresbericht der BAH für 1976.

Mit dem Internationalen Symposium „Ecosystem Research“ vom 26.9. bis zum 1. 10. 1976 auf Helgoland wurde das Ökolabor einer großen Zahl von Wissenschaftlern aus 30 Ländern vorgestellt. $\mathrm{Zu}$ einem geselligen Abend im neuen Haus hatten die Schiffsbesatzungen der BAH ein reichhaltiges, marines und buntes Buffet zubereitet. Die 


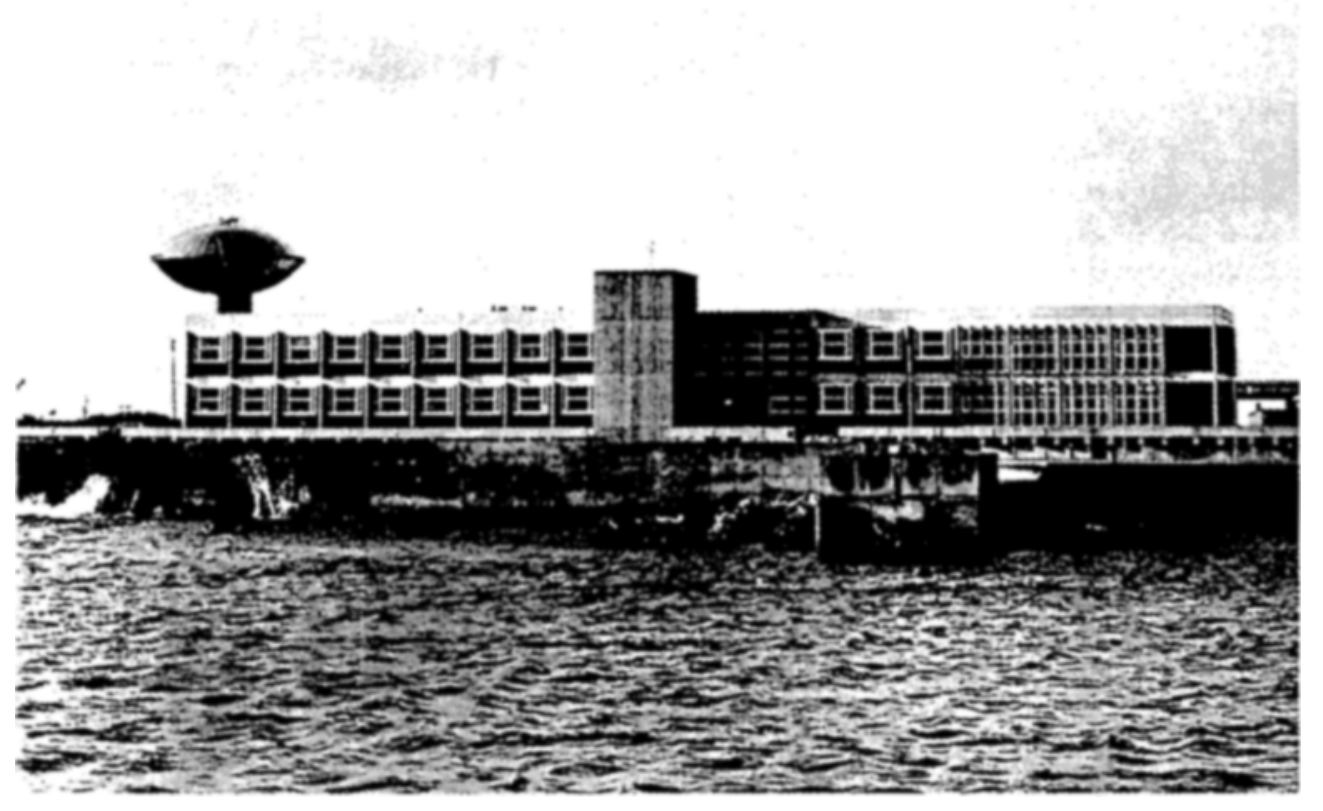

Abb. 36. Das fertige Ökolabor (1976). Photo: Archiv der BAH.

Helgoländer "Karkfinken" sangen Seemannslieder, und die Helgoländer "Skiirskotten " spielten zum Tanz in der Züchtungshalle auf. Die Symposiumsvorträge und -diskussionen, besonders zum Unterthema "Experimental Ecosystems" hatten wie die Vorträge beim Internationalen Symposium 1969 auf Helgoland zum Thema "Cultivation of Marine Organisms and its Importance for Marine Biology " einen direkten Bezug zu den im Ökolabor geplanten Arbeiten, ebenso wie das von Prof. Kinne herausgegebene und zum Teil selbst verfaßte vielbändige Werk "Marine Ecology", die erste umfassende Darstellung über das Leben im Meer.

Eine Gruppe von fünf Wissenschaftlern begann mit ihren Technikern im Ökolabor mit der Arbeit an den im Frühjahr bei Helgoland typischen Planktern: Dr. Ellen Wahl (Ichthyologie), Dr. Klaus Anger (Makrobenthos), Dr. Gotram Uhlig (Mikrofauna), Dr. Wulf Greve (Zooplankton) und Dr. Erik Hagmeier (Phytoplankton). Vom BMFT bewilligte Projektmittel ermöglichten die erforderliche Erweiterung um den Informatiker Dr. Volker Kartheus, einen Verfahrensingenieur, einen Elektroniker und zwei technische Assistentinnen.

Es gab zwei Teilprojekte, (A) Kultivierung ökosystemtypischer Nordseeorganismen (Leiter: Dr. Uhlig) und (B) Analyse populationsregelnder Prozesse durch adaptive Versuchsplanung (Leiter: Dr. Greve), mit jeweils 4 Programmpunkten, die teilweise gleichzeitig bearbeitet wurden. 
Zu (A1) gehörte die Kontrolle von Phosphor-, Stickstoff- und Salzgehalt und des pH der Kulturmedien und der Kreislaufsysteme zur Hälterung von Dorsch, Steinbutt, Scholle und Streifenbarbe zur Laichgewinnung. (A2) umfaßte die Anlage von Futterkulturen aus Flagellaten, Diatomeen, Ciliaten (Fabraea, Eufolliculina), Rädertieren (Brachionus plicatilis) und Muschellarven. Zur Massenproduktion verschiedener Entwicklungsstadien harpacticider Copepoden (Tisbe) entwickelte Dr. Uhlig ein Schwimmsiebverfahren. Die tägliche Produktionsrate von fertilen Weibchen war in Schwimmsieben fast doppelt so hoch und die Mortalität wesentlich geringer als in Standkulturen. Die Anwendung zur Futtergewinnung wurde in deutsch-israelischer Zusammenarbeit geprüft.

In (A3) wurden von Dr. Greve Kulturmethoden für Rippenquallen und Ruderfußkrebse entwickelt. Mit den für die Vermehrung von Flagellaten bewährten Plastikschläuchen von etwa 50 Liter Inhalt wurden Durchflußsysteme zur Naupliengewinnung konstruiert. Polychaeten (Spioniden) wurden über viele Generationen im Labor gehalten (Vera Anger), und es wurde eine Futterautomatik für die Aufzucht von Miesmuschelarven entwickelt (Martin Sprung). Die Aufzucht von Larven der Dekapoden Hyas araneus, Pagurus bernhardus und Carcinus maenas wird seitdem weitgehend beherrscht.

Unter (A4) wurden autökologische Daten über Vermehrungs- und Wachstumsraten bei unterschiedlichen Temperaturen, Lichtverhältnissen, Ernährungsbedingungen und Wasserbewegungen gewonnen. Dabei wurden die Generationszeiten für zahlreiche einzellige Algen des Helgoländer Planktons bestimmt. Ein Vergleich der Optima mit dem Auftreten größerer Zahlen dieser Arten in situ ergab, daß Maxima und Sukzessionen meistens durch den Einfluß von Licht und Temperatur zu deuten sind (Dr. Hagmeier). Umfangreiche Untersuchungen galten dem Meerleuchttierchen Noctiluca scintillans, seiner Reproduktionsrate und der Dauer der Zellteilung in Abhängigkeit von Temperatur und Salzgehalt, verbunden mit Beobachtungen über die Populationsdynamik in der Deutschen Bucht (Dr. Uhlig, Gerrit Sahling). Bei den Decapoden und Cirripediern konnten nach der Vervollkommnung der Hälterungstechnik Energiebudgets für verschiedene Larvenstadien erstellt und der Ablauf der Häutungszyklen untersucht werden (Dr. Anger, Joachim Harms). Es ergaben sich zahlreiche Themen für Diplomarbeiten und Dissertationen über die Autökologie meroplanktischer Organismen und die intensive Zusammenarbeit der BAH mit dem Centro de Biologia Marinha, Curitiba (Brasilien).

Zu den Arbeiten im Rahmen des zweiten Teilprojekts gehörten (B1) die Einrichtung einer Datenbank und die Erstellung eines Simulationsmodells, (B2) die Ermittlung von wichtig erscheinenden Parametern aus diesem Modell sowie eine entsprechend orientierte adaptive Versuchsplanung, (B3) kurzzeitige Versuche über Wechselwirkungen zwischen Organismen und Populationen und längere Zeitreihen über Massenverschiebungen beim Zusammenleben verschiedener Arten sowie ein Bezug zu den Verhältnissen in der Deutschen Bucht durch "systemökologische Meßfahrten". (B4) betraf schließlich die Verarbeitung der Daten zu einem besser angepaßten Modell und einer erneuten adaptiven Versuchsplanung. So konnten zum Beispiel die Vorstellungen über die Wechselwirkungen zwischen dem Ruderfußkrebs Calanus helgolandicus und der Rippenqualle Pleurobrachia pileus und zwischen den Rippenquallen Pleurobrachia und Beroe gracilis durch Versuche im Labor und wiederholte Beobachtungen auf See optimiert werden.

Im Jahr 1980 besuchte Bundespräsident Dr. Carstens in Begleitung von Ministerpräsident Stoltenberg (Abb. 37) die BAH und insbesondere das Ökolabor. Für Information 


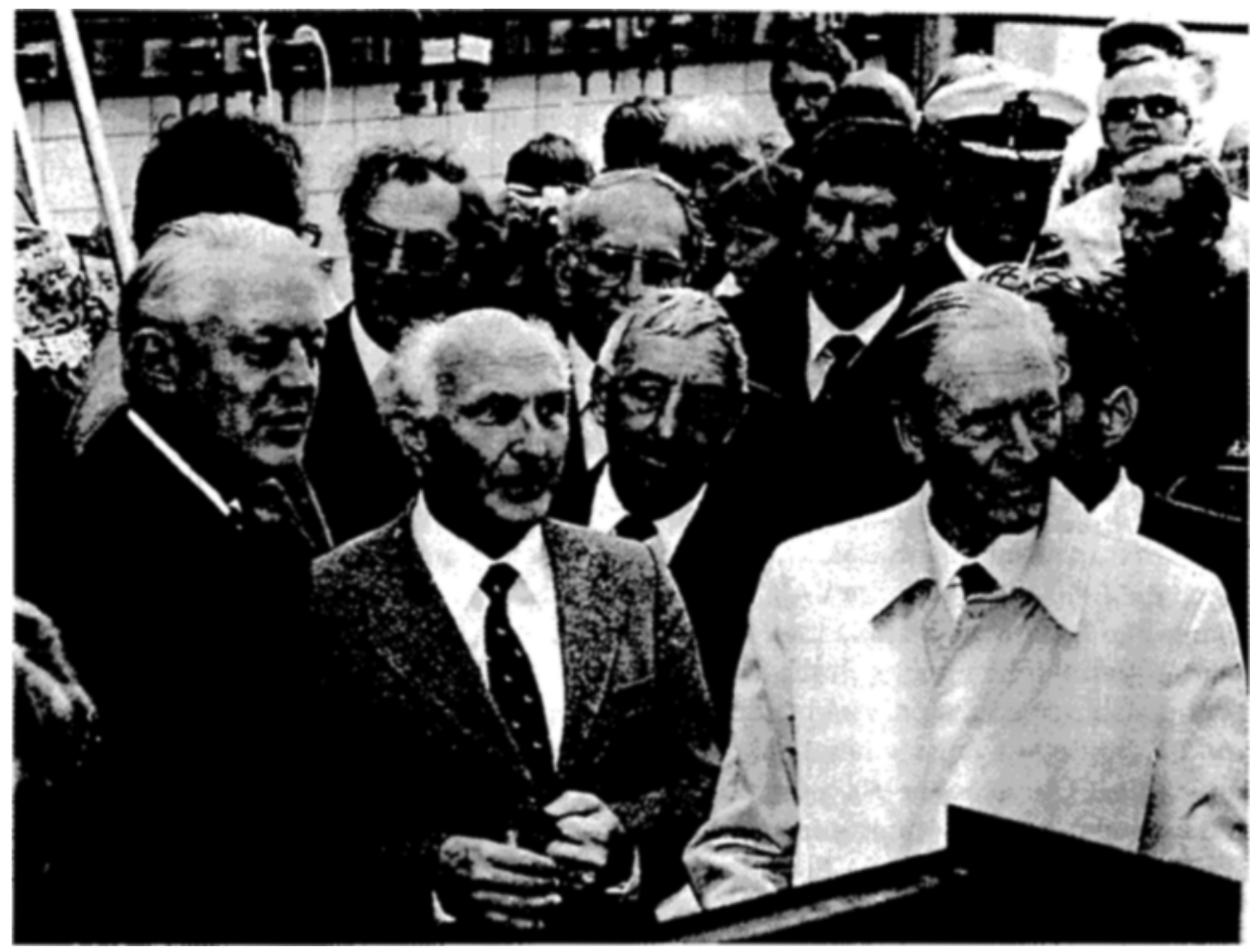

Abb. 37. Besuch im Ökolabor im Jahr 1980. Vordere Reihe von links nach rechts: Ministerpräsident Stoltenberg, Dr. Uhlig, Bürgermeister Rickmers, Bundespräsident Dr. Carstens. Photo: Gerrit Sahling.

und Verbindung an der Meeresstation sorgte von 1978 bis 1981 das "Ökojournal“ . Es enthielt "Kulturnachrichten“ über die jeweils verfügbaren Stämme von Einzellern, Beobachtungen, etwa über Dorsche, die beim Ablaichen über das Wasser springen, Saat- und Kanadagänse auf dem Ökolabor-Rasen im Januar 1979 und Seehundsnachwuchs. Methoden für chemische Analysen, Filtration, Zählungen und Lichtmessungen wurden beschrieben, Ergebnisse über Seespinnen- und Miesmuschelzucht mitgeteilt. Außerdem wurden neue Mitarbeiter, Kurse und Gastforscher vorgestellt. Es gab Berichte von Konferenzen und Reisen, etwa über die Mesokosmos-Untersuchungen mit der "Friedrich Heincke" im Loch Ewe oder die Züchtung von Warmwasser-Decapoden in North Carolina. Marktberichte deuteten das Rufzeichen der „Friedrich Heincke“, nämlich DBFK = Die Bio Fischte Kaum, oder das Rufzeichen der "Uthörn", DBFN = Die Bio Fischte Nichts, und schließlich gab es auch meeresbezogene Poesie von Eugen Roth und Franz Dingelstedt, etwa über das Meeresleuchten.

Nach Auslaufen des durch das BMFT über knapp fünf Jahre geförderten ÖkolaborProjektes wurden die einzigartigen Einrichtungen des Ökolabors vor allem für Arbeiten mit Decapoden und Amphipoden von Dr. Klaus Anger, Dr. Hans-Dieter Franke und Dr. Joachim Harms mit zahlreichen Examenskandidaten genutzt. 


\section{Bibliothek und Veröffentlichungen der BAH}

Mit dem Ökolabor entstand auf Helgoland auch ein zweckmäßiger und repräsentativer Raum für die wieder umfangreich gewordene Bibliothek. In Hamburg bekam die Bibliothek in Neubau der Zentrale einige Jahre später ebenso ein angemessenes Zuhause. Durch den Zweiten Weltkrieg und seine Folgen war der Bestand der BAH an Büchern, Zeitschriften und Separaten, damals vergleichbar mit der Bibliothek der Zoologischen Station in Neapel, fast vollständig verloren gegangen. Die geborgenen Reste wurden in der Station in Sylt auf dem Ellenbogen aufgestellt.

Im Jahr 1950 konnte die Reihe der "Helgoländer Wissenschaftlichen Meeresuntersuchungen" (HWM) mit Band 3 fortgesetzt werden. Es wurden Arbeiten veröffentlicht, zu denen das Material vor dem Zweiten Weltkrieg gesammelt worden war. Die nächsten, von 1952 bis 1956 erscheinenden Hefte der Bände 4 und 5 wurden zugleich als "Beihefte 1-6“ der Berichte der Deutschen Wissenschaftlichen Kommission für Meeresforschung bezeichnet. Die nächsten Bände erschienen wieder in schnellerer Folge. Sie wurden durch die Beiträge der an der BAH veranstalteten Symposien bereichert. Mit den HWM konnte die BAH etwa zwei Drittel der in der Bibliothek gehaltenen periodisch erscheinenden Veröffentlichungen durch Tausch erwerben (Renckhoff, 1992).

Im Institutsneubau auf Helgoland war zunächst ein Raum über dem großen Kurssaal für die Bibliothek ausreichend. In Hamburg wurde der durch Zeitschriften-Tausch (gegen die "Helgoländer Wissenschaftlichen Meeresuntersuchungen") und durch Sonderzuwendungen des Ministeriums wachsende Bestand getrennt von den Büchern der Bundesforschungsanstalt für Fischerei aufgestellt. Erst 1966 bekam der Bibliothekar Werner Meiss eine Hilfskraft. Im Jahr 1969 wurde die Bibliothek erstmals in einem besonderen Abschnitt im Jahresbericht der BAH erwähnt. Der Bestand war auf 2800 Bücher, 11000 Zeitschriftenhefte und 15500 Sonderdrucke gestiegen.

Der weitere Ausbau der Bibliothek auf Helgoland wurde angestrebt. Der Nachfolgerin von Dipl.-Bibl. Werner Meiss, Dipl.-Bibl. Inka Schritt (später Renckhoff) blieb es vorbehalten, diesen Ausbau ab 1971 zu gestalten. Es war das Ziel von Prof. Kinne, die Möglichkeit zu konzentrierter Arbeit auf der Insel auch für Literaturstudien durch eigene Wissenschaftler und Gastforscher in einer gut ausgestatteten Präsenz-Bibliothek zu nutzen, so wie er es in Woods Hole kennengelernt hatte. Raum und Bestand im Ökolabor boten nun dafür die besten Voraussetzungen.

Prof. Kinne beschaffte nicht nur die Mittel zum Ausbau der Bibliothek. Durch die Begründung und Herausgabe von englischsprachigen Zeitschriften und Buchreihen sorgte er dafür, daß die Ergebnisse meeresbiologischer Arbeit in internationalem Rahmen veröffentlicht werden konnten. So enstand zunächst die Zeitschrift Marine Biology (International Journal on Life in Oceans and Coastal Waters). Sie wurde von Prof. Kinne im Jahr 1969 in Zusammenarbeit mit dem Springer-Verlag gegründet, schon im September 1994 wurde Band 120 publiziert. Marine Biology entwickelte sich rasch zu einer führenden Zeitschrift der meeresbiologischen Forschung in aller Welt. Als Buchreihe enstand Marine Ecology (A Comprehensive, Integrated Treatise on Life in Oceans and Coastal Waters) verlegt von John Wiley \& Sons, Chichester. Dieses von Prof. Kinne 1970-1984 herausgegebene Handbuch behandelt die fünf Teile Environmental Factors, Physiological Mechanisms, Cultivation, Dynamics und Ocean Management und vermittelte auf zusammen 7031 Seiten einen zusammenfassenden und kritischen Überblick 
über den Stand unserer Kenntnisse von Organismen und ökologischen Beziehungen im Meer und in den Küstengewässern. Ab 1979 folgte die Zeitschrift Marine Ecology Progress Series (MEPS), verlegt vom Verlag Inter Research, Oldendorf/Luhe, mit über 100 Beratern der Herausgeber sowie 400 Referenten und einer Produktion von 115 Bänden bis Dezember 1994. Schließlich erschien im Zeitraum 1980-1990 die Buchreihe "Diseases of Marine Animals", verlegt von John Wiley \& Sons und zum Teil von der BAH. Es handelt sich um eine Zusammenstellung der Krankheiten und Abnormitäten von marinen Einzellern bis zu marinen Säugetieren, mit vier Bänden von zusammen 2618 Seiten.

Ende 1977, so hieß es im Jahresbericht, verfügte die Bibliothek über 5761 Monographien und 24992 gebundene Zeitschriftenbände. Mit den Sonderdrucken zusammen waren das 56664 bibliographische Einheiten. Es wurden 720 Periodica gehalten, wovon 210 durch Kauf erworben waren, der Rest durch Tausch gegen die "Helgoländer wissenschaftlichen Meeresuntersuchungen“. Im Jahr 1977 gab es 5850 Literaturanforderungen aus dem Kreis von Institutsmitarbeitern, Gastforschern und Benutzern des Leihverkehrs. Davon konnten aus eigenem Literaturbestand $63 \%$ gedeckt werden. Die umfangreichen Bibliotheksarbeiten an den drei Standorten mußten von Frau Schritt mit vier Mitarbeitern bewältigt werden. Seit 1980 redigiert Frau Carol Berger die „Helgoländer Meeresuntersuchungen" (HM), die bis 1978 "Helgoländer wissenschaftliche Meeresuntersuchungen" hießen. Im Jahr 1981 bekam auch die Bibliothek in Hamburg größere Räume im Neubau der Zentrale. Die Zahl der zu betreuenden Einheiten stieg 1982 auf 82946 und die der Literaturanforderungen auf 11 012, ohne daß zusätzliche Mitarbeiter eingestellt werden konnten. Es wurden 715 Periodica gehalten, wobei die wegen beschränkter Finanzmittel notwendige Auswahl wegen der weit gefächerten Arbeiten der Wissenschaftler an der BAH auf den Gesamtkonferenzen oft zum heiß diskutierten Thema gerieten.

Im Jahr 1986 wurde eine neue Veröffentlichungsreihe begonnen, die "Berichte der Biologischen Anstalt Helgoland ". In dieser Reihe sollten in Manuskriptform datenreiche Arbeiten aus dem Institut rasch verbreitet werden. Die ersten beiden Hefte enthielten eine quantitative Abschätzung des partikulär und gelöst vorhandenen Stickstoffs in der Deutschen Bucht sowie eine Darstellung der Helgoland-Reede-Daten von 1962 bis 1984 über Nährstoffe und Phytoplankton.

Die Leiterin der BAH-Bibliothek, Frau Inka Renckhoff, gründete im Jahr 1990 die "Arbeitsgemeinschaft meereskundlicher Bibliotheken", die sich zweimal im Jahr trifft. Die BAH-Bibliothek ist Mitglied in nationalen und internationalen Arbeitsgemeinschaften, so zum Beispiel bei EURASLIC (European Association of Aquatic Science Libraries and Information Centres) und IAMSLIC (International Association of Aquatic and Marine Science Libraries and Information Centres). Die BAH weist nach dem Bundesamt für Seeschiffahrt und Hydrographie den größten Bestand an mariner Literatur auf, mit 120239 bibliographischen Einheiten Ende 1991, von denen sich der überwiegende Teil in der Hamburger Zentrale befindet. Auf Helgoland und in List wurde zusätzlich Literatur für Gastforscher und Kurse bereitgestellt. Als eine der wenigen Hamburger Bibliotheken nimmt die Bibliothek der BAH am überregionalen Leihverkehr der Bibliotheken teil.

"Aus der kleinen beschaulichen Bücherei der Königlichen Biologischen Anstalt auf Helgoland " so schrieb die Leiterin der Bibliothek zum Institutsjubiläum im Jahr 1992, "ist ein Glied eines modernen meeresbiologischen Informationssystems geworden " (Renck- 
hoff, 1992). Und doch spürt man noch etwas von vergangenen Zeiten in der BAH-Bibliothek. Einige der von Prof. Heincke in seinem ersten Jahresbericht erwähnten Bände sind noch erhalten, viele Zeitschriftenreihen reichen bis in das 19. Jahrhundert zurück, und an den Bibliotheksstempeln mit wechselnden Insignien läßt sich der Weg des Instituts von der Königlichen Biologischen Anstalt bis zur Biologischen Anstalt Helgoland in Helgoland, List und Hamburg verfolgen.

\section{Neubau der Zentrale Hamburg und Verabschiedung von Prof. Kinne}

Nach der Übernahme durch den Bundesminister für Bildung und Wissenschaft im Jahr 1971 war die BAH bestrebt, sich auch räumlich von der Bundesforschungsanstalt für Fischerei zu trennen, um auch in Hamburg mehr Platz für Forschung und Verwaltung zu gewinnen. Prof. Kinnes langjährige Bemühungen um ein Grundstück für den Neubau hatten im Jahr 1974 Erfolg. An der Notkestraße in Hamburg-Groß-Flottbek wurde auf dem Gelände des Deutsches Elektronen-Synchrotons (DESY) Baugrund zur Verfügung gestellt. Die Baupläne wurden nach dem Bedarf der BAH von der Abteilung Universitätsbauten der Baubehörde der Freien und Hansestadt Hamburg erarbeitet. "Bei einer Nettogrundrißfläche von $6195 \mathrm{~m}^{2}$ wurde ein dreigeschossiges Gebäude mit einem Bibliothekstrakt und einer Hausmeisterwohnung konzipiert. Im Spätsommer 1978 wurden die ersten vorbereitenden Baumaßnahmen in Angriff genommen. Die Erstellung des Rohbaus ging zügig voran, so daß am 18. Dezember 1979 das Richtfest stattfinden konnte“. So wurde die neue Zentrale im Jahresbericht der BAH für 1979 vorgestellt.

Der Neubau (Abb. 38) wurde am 29.10.1981 der BAH zum Einzug übergeben und am 6. 5. 1982 feierlich in Dienst gestellt. Prof. Sebastian Gerlach (IfM Kiel) hielt den Festvortrag über das Thema: „Verändern sich die Lebensbedingungen in deutschen Küstenmeeren?" Er machte deutlich, in welchem Maße natürliche Veränderungen stattfinden und betonte, wie schwierig und langwierig es ist, die Wirkung von menschlichen Einflüssen dabei zu erkennen. Die Mittel für das Bauvorhaben von rund $20 \mathrm{Mio.} \mathrm{DM} \mathrm{kamen}$ vom Bundesminister für Forschung und Technologie (Bulnheim, 1983; Kurzweg, 1984).

Das Erdgeschoß des Neubaus enthält die Diensträume des Direktors und der Verwaltung, den Sitzungs- und Aufenthaltsraum, die Bibliothek, Werkstätten und Garagen. Im Keller sind die Betriebstechnik, Kultur-, Lager- und Schutzräume untergebracht. Drei Seewasser-Kreislaufsysteme basierten auf den Ergebnissen langjähriger Untersuchungen zur Betriebsoptimierung von Kreislaufanlagen mit nahezu vollständiger Wasseraufbereitung. Im Jahresbericht für 1982 wurden Details erklärt. Zu jedem Kreislauf gehörten Einrichtungen für Sedimentation von Schmutzstoffen, biologische Aufbereitung, Belüftung sowie Temperaturregelung, zur Ozonisierung, zum Ausgleich von Verdunstungsverlusten sowie Hoch- und Tiefbehälter. Für Versuche in größerem Maßstab mit biologisch-chemischen Aufbereitungsverfahren zur Aufzucht von marinen Nutztieren wurde 1984-1985 noch eine Halle mit $260 \mathrm{~m}^{2}$ Grundfläche hinter dem Institut errichtet.

In den über dem Erdgeschoß liegenden Stockwerken der Zentrale Hamburg der BAH wurden die Laboratorien und Diensträume der Wissenschaftler mit verschiedenen Spezialeinrichtungen wie Isotopenlabor, Massenspektrometer und Elektronen-Mikroskop untergebracht. Außerdem gab es hier Laboratorien für Gastforscher und bis 1992 auch Büroräume für die Stiftung "Jugend forscht e.V.". 


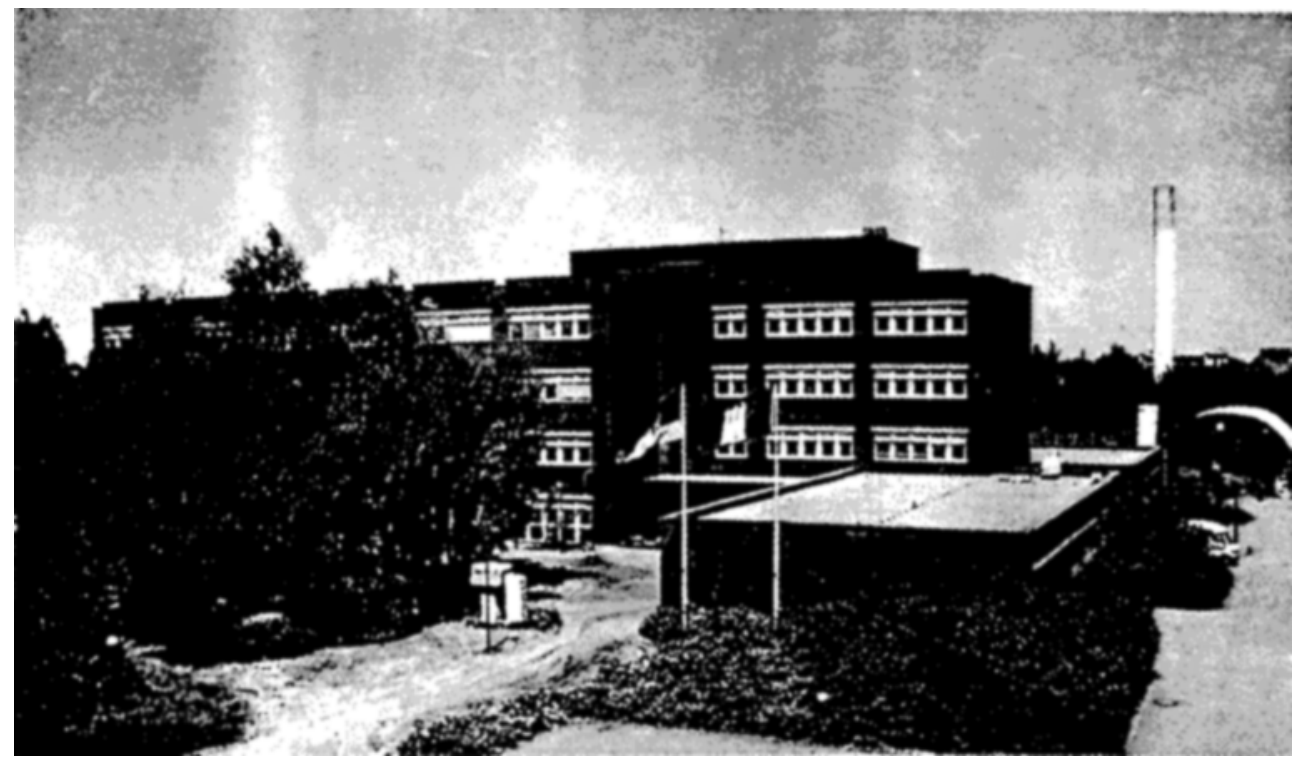

Abb. 38. Die Zentrale der BAH, Notkestraße 31, in Hamburg. Photo: Archiv der BAH.

Die Organisation und die Personalaufstellung der BAH für das Jahr 1982 werden auszugsweise in Tabelle 3 gezeigt.

Ende Juni 1984 trat Prof. Kinne nach 22jähriger Tätigkeit als Direktor der BAH wegen einer zunehmenden Schwerhörigkeit in den Ruhestand. Die Deutsche Zoologische Gesellschaft verlieh ihm die Karl-Ritter-von-Frisch-Medaille und den Wissenschaftspreis der Gesellschaft für seine umfassenden Untersuchungen und Veröffentlichungen auf dem Gebiet der Meeresökologie. Die BAH wurde unter seiner Führung zu einem der wichtigsten meeresbiologischen Forschungsinstitute der Welt ausgebaut (Verleihungsurkunde).

\section{Kurzüberblick 1984-1994}

Ab Juli 1984 übernahm Prof. Bulnheim (Abb. 39) als Vertreter des Direktors die Leitung der BAH. Im Mai 1988 erkrankte Prof. Bulnheim, und die stellvertretende Leitung ging auf Prof. Lüning (Abb. 40) über, ab 1.7.1989 auf Prof. Gunkel (Abb. 41), der am 11.9. 1990 zum Direktor der BAH ernannt wurde und am 30.9.1993 in den Ruhestand trat. Dr. Hoppenheit übernahm dann die Vertretung des Direktors, und am 1.4.1994 wurde Wilhelm Nultsch (Abb. 40), Professor der Universität Marburg und langjähriger BAH-Gastforscher, zum kommissarischen Direktor der BAH ernannt.

Nachfolger von Prof. Bulnheim (Hamburg) als Leiter der Abteilung Meereszoologie wurde am 1.4.1992 Prof. Friedrich Buchholz (Helgoland), Nachfolger von Prof. Gillbricht (Hamburg) als Leiter der Abteilung Biologische Ozeanographie am 26. 9. 1991 Prof. Karsten Reise (List). Die Leitung der Abteilung Experimentelle Ökologie, bis zum 30.4. 1992 Dr. Uhlig (Helgoland), wurde anschließend von Dr. Hoppenheit und später von Prof. Nultsch wahrgenommen. Nachfolger von Prof. Gunkel (Helgoland/Hamburg) als Leiter 


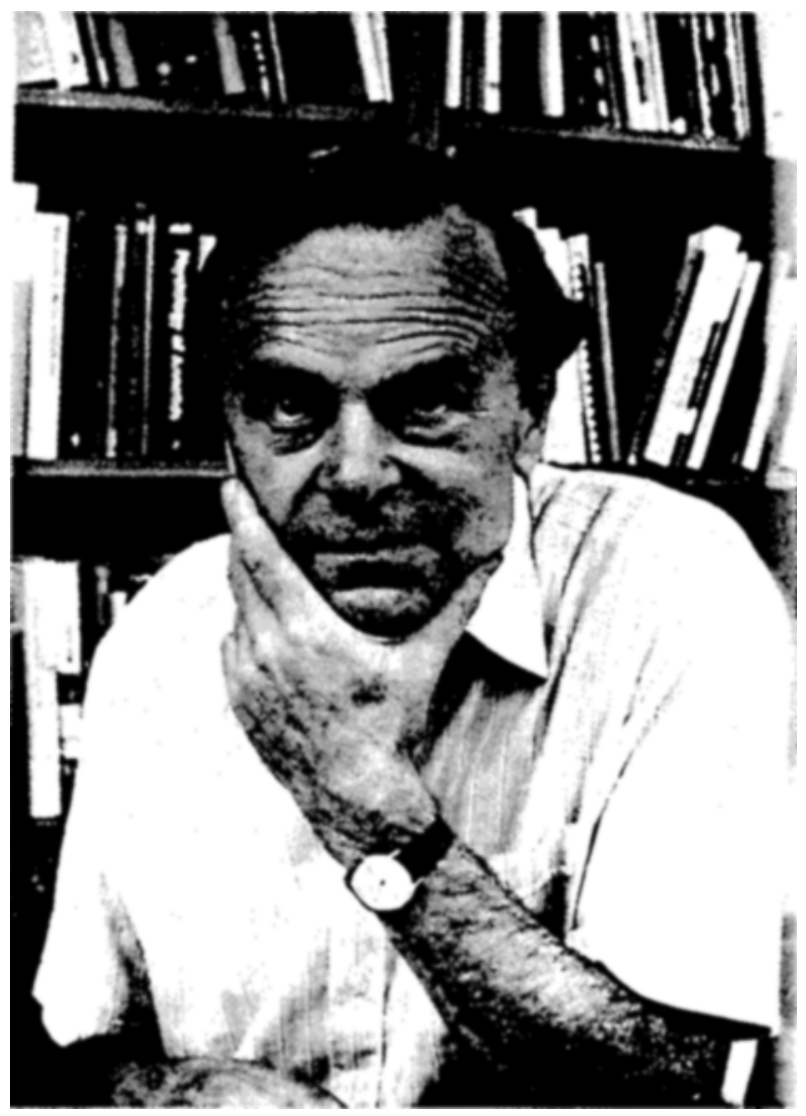

Abb. 39. Prof. Dr. Hans-Peter Bulnheim, 1989. Photo: Archiv der BAH.

der Abteilung Meeresmikrobiologie wurde am 1.7.1992 Dr. Christian Schütt (Helgoland).

Im Beirat der BAH übernahm Prof. Wilhelm Nultsch (Marburg) im Jahr 1987 den Vorsitz, Prof. Ax (Göttingen) wurde sein Stellvertreter, und Prof. Dietrich Neumann (Köln) wurde als neues Mitglied berufen. Ab 1.4.1991 wurden Prof. Gotthilf Hempel (Bremerhaven; Vorsitzender), Prof. Anthony Chapman (Kiel) und Prof. Gerd Hubold (Hamburg) als neue Mitglieder ernannt, ab 1.7.1992 Prof. Detlef Bückmann (Ulm; ein Sohn von Prof. Adolf Bückmann), Dr. Kurt Fedra (Laxenburg, Österreich), Prof. Albrecht Fischer (Mainz; Vorsitzender), Prof. Peter Fortnagel (Hamburg), Prof. Winfried Gieskes (Groningen) und Prof. Dietwart Nehring (Rostock-Warnemünde).

Nach dem Auftreten von sauerstoffarmem Bodenwasser in der Deutschen Bucht und Schäden an der Bodenfauna 1981-1983 begannen 1984 vom BMFT geförderte Untersuchungen der möglichen Ursachen durch die Arbeitsgruppe Dr. Eduard Bauerfeind, Dr. Hickel, Ulrich Niermann und Dr. von Westernhagen. Auf einem Stationsnetz in der Deutschen Bucht und nördlich davon bis zur Kleinen Fischerbank wurden Wasser-, Planktonund Benthosproben genommen; eine erneute gravierende Sauerstoffarmut war zunächst 


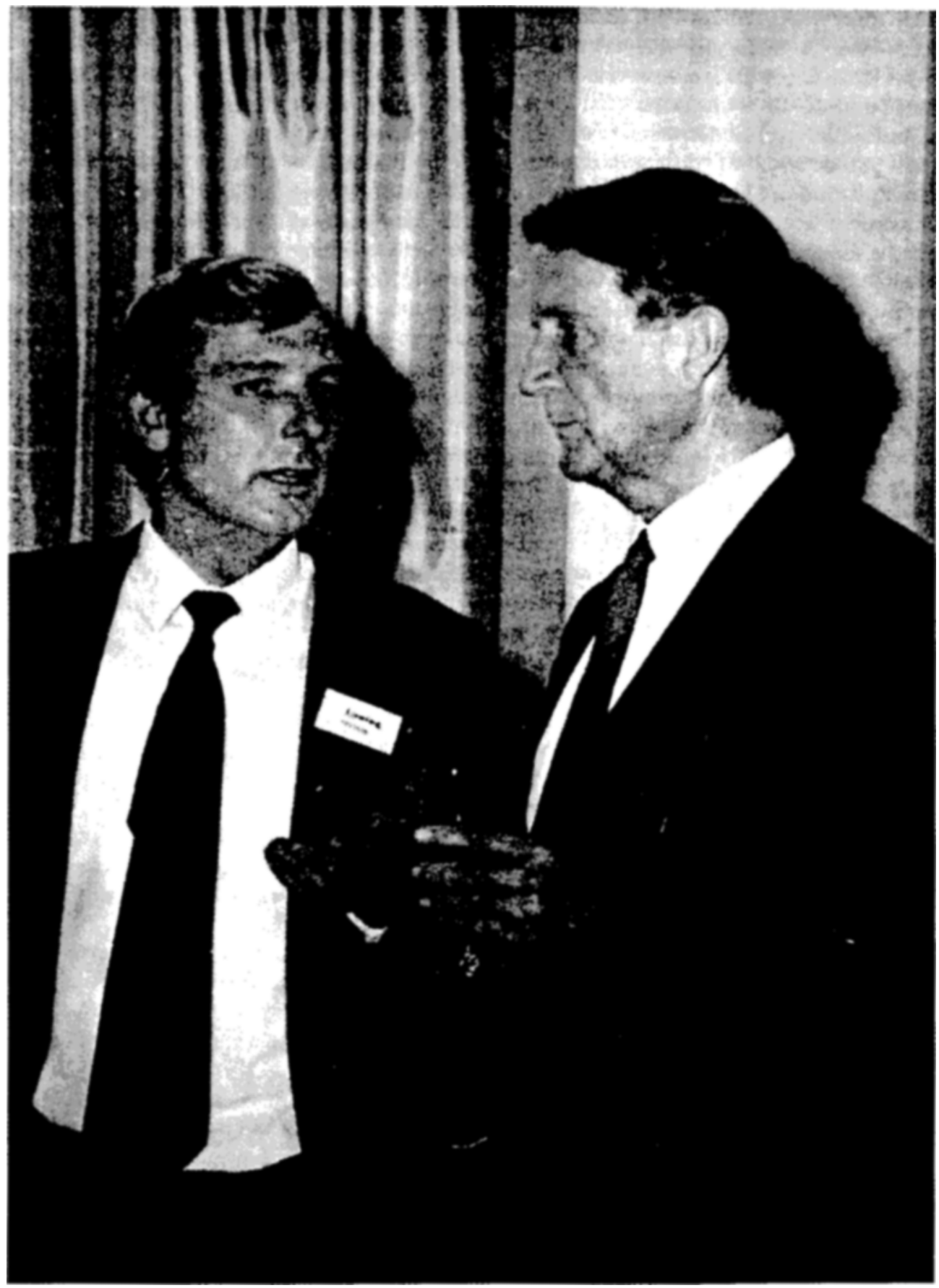

Abb. 40. Botaniker im Gespräch, 1987. Prof. Klaus Lüning (links) und Prof. Wilhelm Nultsch (Marburg), der spätere Direktor der BAH. Photo: Archiv der BAH. 
nicht zu messen, sondern ein Ansteigen von Arten- und Individuenzahlen im vorher geschädigten Benthos. Es wurden das Entstehen, Verdriften und Absinken von Phytoplankton-Konzentrationen beobachtet (es gab "Blüten" in den Frühjahren 1985 und 1986) und die Bedeutung der Konvergenzzonen (Fronten) für diese Vorgänge; der Sauerstoffbedarf von Bodenwasser und Sediment wurden gemessen. Erst im Spätsommer 1989 gab es wieder kritische Sauerstoff-Werte im Bodenwasser, deren Verlauf mit Forschungsschiffen dänischer Institute, des DHI, des Alfred-Wegener-Instituts und der BAH untersucht werden konnten: die sauerstoffarme Zone erstreckte sich in Nord-Süd-Richtung von Höhe Ringkøbing Fjord bis zur Höhe von Sylt entlang der 40-m-Tiefenlinie. Die Fische wanderten ab, mit der Dredge wurden rund 10mal mehr Herzigel gefangen als in vergleichbaren Gebieten ohne Sauerstoffmangel.

Seit 1983 werden zusammen mit der Bundesforschungsanstalt für Fischerei Mißbildungs- und Schlupfraten pelagischer Eier von Fischen in der Ostsee und in der südlichen Nordsee untersucht, 1991 in einem bis in die zentrale Nordsee erweiterten Stationsnetz. Dabei gibt es Unterschiede von Art zu Art, aber für alle ein Ansteigen der Störungen während der letzten Jahre und Häufungen vor der Rheinmündung und im Osten der Deutschen Bucht (Patricia Cameron, Dr. von Westernhagen, Joachim Berg mit Dr. Dethlefsen von der BFAFi).

In die Untersuchungen über Einflüsse der Meeresverschmutzung wurden die langjährigen Benthosuntersuchungen der BAH einbezogen, die Dr. Ziegelmeier nach dem 2. Weltkrieg weiterführte. In neuerer Zeit gibt es eine Studie der Fluktuationen im Makrobenthos der südöstlichen Nordsee 1983-88 (Niermann, 1997). Darin zeigt ein Langzeitvergleich die Tendenz eines Anstiegs der Gesamtbiomasse seit den Erhebungen am Beginn und in der Mitte dieses Jahrhunderts westlich von Amrum und Sylt um den Faktor 1,4 bis 2. Die seit 1924 laufenden Arbeiten der BAH über Lebensgemeinschaften und Lebensbedingungen im Wattenmeer wurden 1986 unter der Leitung von Prof. Karsten Reise in der interdisziplinären Projektgruppe "Wattenmeerökologie" intensiviert, nach der Veröffentlichung seines Buches "Tidal Flat Ecology" (Reise, 1985). Bei einem Bestandsvergleich im Benthos der Norderaue (zwischen Föhr und Langeneß) zwischen 1923-1926 und 1985 zeigte sich, daß die damaligen Austernbänke verschwunden und die Sabellaria-Riffe zerstört sind, die Miesmuschelbänke hatten sich ausgedehnt und die Endofauna hatte zugenommen. An einer Stelle bei List im Nordsylter Wattenmeer waren früher ebenfalls die meisten sessilen Arten der Epifauna signifikant häufiger (Zerstörungen durch Bodennetze werden als Ursache angenommen), einige fehlen heute ganz (Ostrea), bei den mobilen Formen gibt es nur wenig Veränderungen, der Rotalgenbestand hat im Sublitoral abgenommen. Endo- und Epibenthos im Verklappungsgebiet der Titanindustrie wurden noch vor dem Einbringungsstopp 1989 untersucht: Es ist nicht sicher nachzuweisen, daß Artenvielfalt und Abundanz generell beeinträchtigt wurden.

Im September 1988 fand das 6. Internationale Wattenmeersymposium "Monitoring the Wadden Sea" auf Sylt statt; die Vorträge der Teilnehmer aus den drei Wattenmeerländern wurden in den Helgoländer Meeresuntersuchungen, Band 43 (1989) veröffentlicht. Seit 1989 gehört das Projekt "Wattenmeerökologie“ zu dem Großvorhaben "Ökosystemforschung Wattenmeer, Schleswig-Holstein “. Es besteht aus (A) einem geographisch-historischem Ansatz unter Betonung von Umweltproblemen: "Strukturelle Ökosystemforschung, ökologische Umweltbeobachtung " und (B) einem prozeßorientier- 
ten Ansatz: "Sylter Wattenmeer Austauschprozesse" (SWAP). Gemeint ist das Wattenmeer zwischen dem Nordteil der Insel Sylt, dem Hindenburgdamm, dem Festland, dem Römödamm und dem Südteil der Insel Römö. In beiden Teilen ist die Projektgruppe der Biologischen Anstalt tätig und fungiert außerdem für (B) als organisatorisches Zentrum (Boley et al., 1992). Schließlich wurden seit 1994 im Rahmen des BMBF-Projekts TRANSWATT (Transport und Transformation von Biomasse-Elementen in Wattgewässern) Phasenübergänge der Elemente $\mathrm{P}, \mathrm{N}$ und $\mathrm{C}$ in der Wassersäule untersucht, besonders die Bindungsformen und Verfügbarkeit des Phosphats (Dr. Hickel).

Wissenschaftlerinnen und Wissenschaftler der BAH wirken auf Ersuchen der Bundesminister für Forschung und Technologie, für Verkehr wie für Umwelt, Naturschutz und Reaktorsicherheit, des Bundesamtes für Seeschiffahrt und Hydrographie, des Umweltbundesamtes, des Bergamtes Meppen und der Bund/Länder-Arbeitsgemeinschaft Nordsee-Küstengewässer durch ihren fachlichen Rat und ihre Tätigkeit mit bei der Umsetzung und Fortentwicklung der Konventionen zum Schutz des Meeres. Schließlich müssen an dieser Stelle die zahlreichen und umfangreichen Beiträge und die redaktionelle Beteiligung von Mitarbeiterinnen und Mitarbeitern der BAH in den von Dr. José Lozán initierten "Warnsignale"-Büchern erwähnt werden (Lozán et al., 1990; Lozán et al., 1994; Lozán \& Kausch, 1996; Lozán et al., 1996).

Im August 1989 besuchten als prominente und interessierte Gäste Bundespräsident Richard von Weizsäcker und seine Gattin die Meeresstation Helgoland (Abb. 41 und 42).

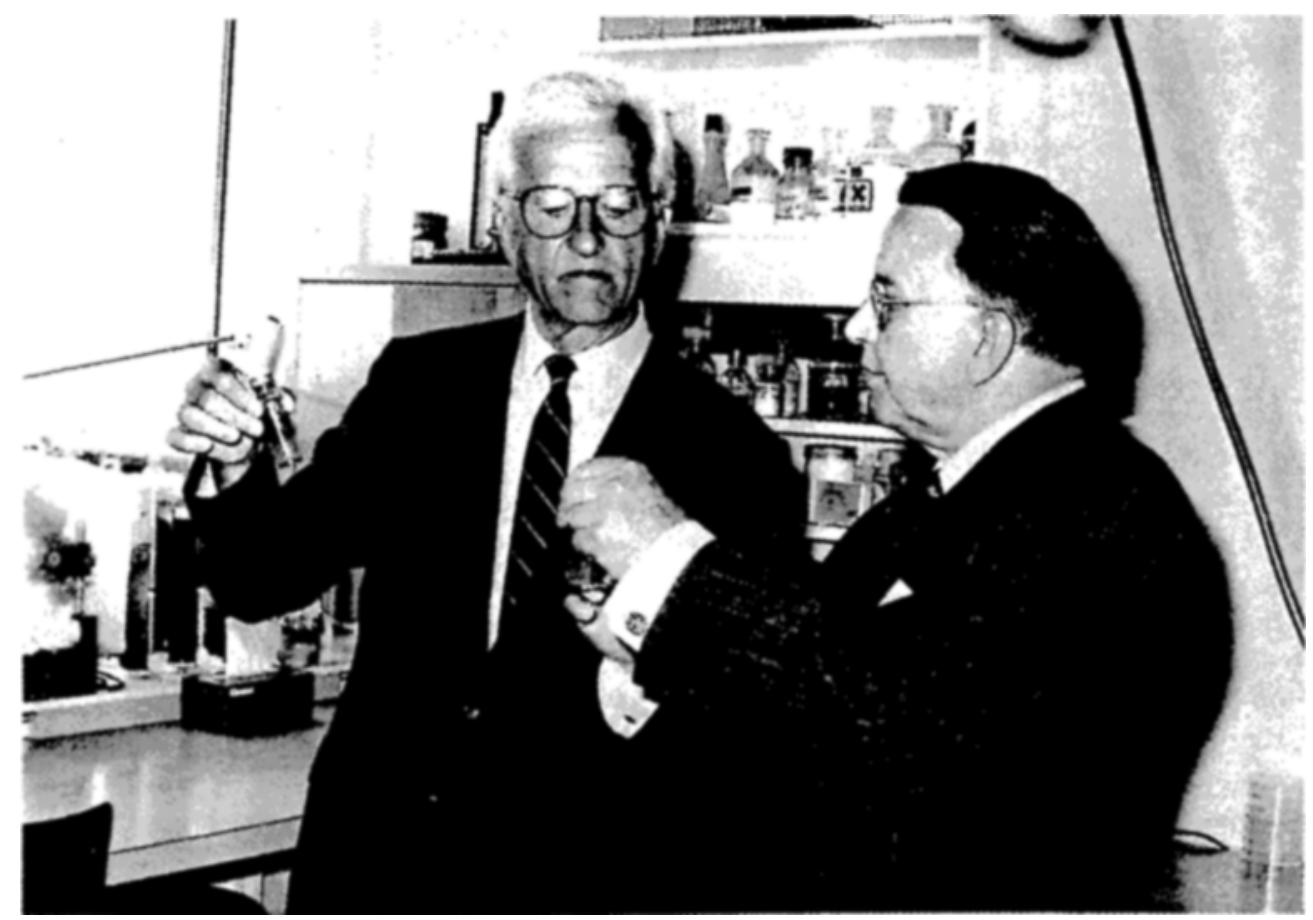

Abb, 41. Prof. Wilfried Gunkel erläutert dem Bundespräsidenten Dr. Richard von Weizsäcker (links) den mikrobiellen Ölabbau (15.8. 1989). Photo: Gerrit Sahling. 


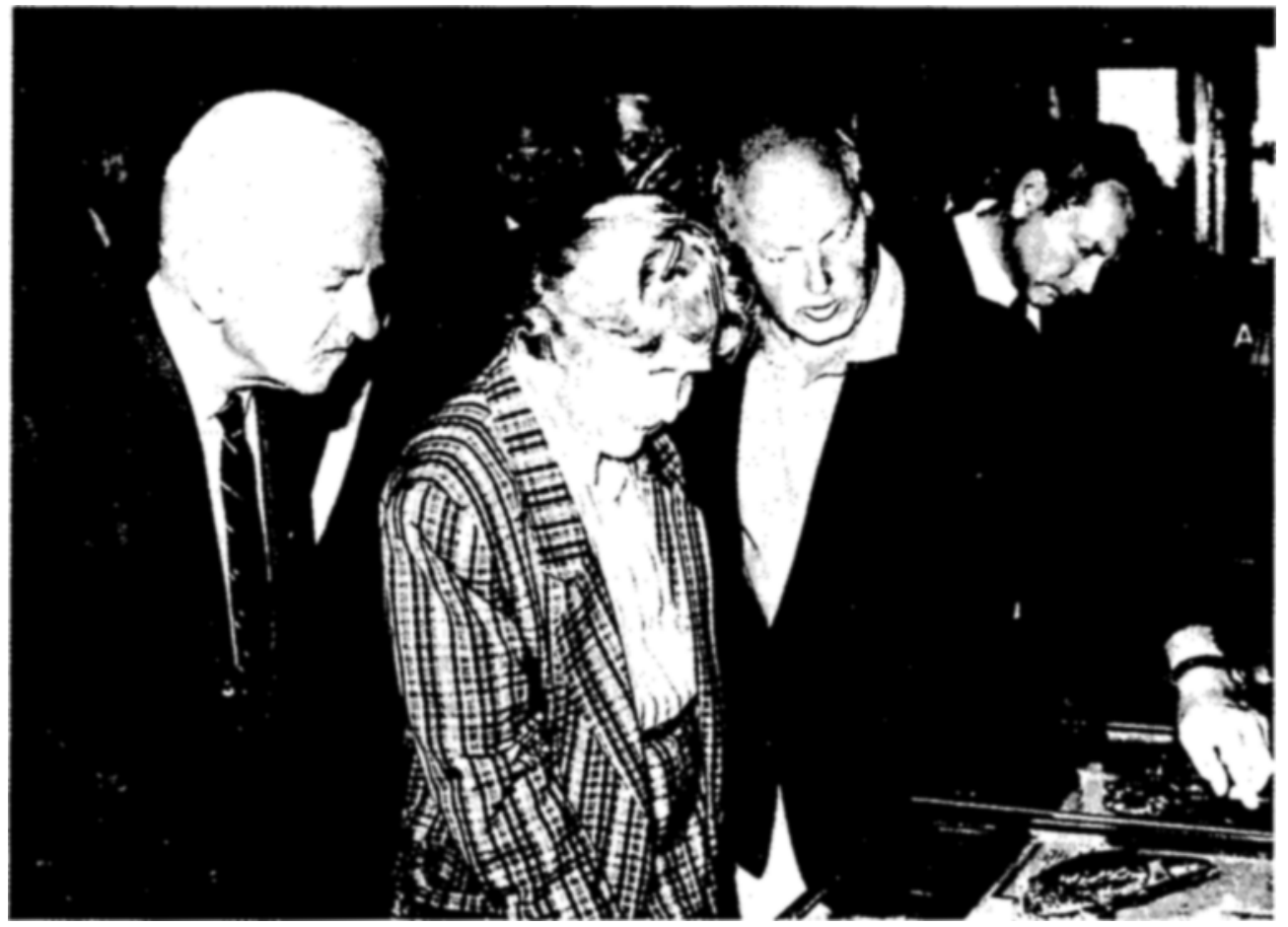

Abb. 42. Besuch im Aquarium im Jahr 1989. Olaf Goemann erzählt Frau Marianne von Weizsäcker und dem Bundespräsidenten Dr. Richard von Weizsäcker etwas vom Helgoländer Hummer. Photo: Gerrit Sahling.

Am 8.6. 1990 wurde das bisher größte Forschungsschiff, die "Heincke“ (Abb. 43), auf einer Weserfahrt an den Bundesforschungsminister und von diesem an den Direktor der $\mathrm{BAH}$ übergeben. Das Vorgängerschiff "Friedrich Heincke" wurde verkauft und verließ am 26. 5. 1990 den Helgoländer Hafen, um von ihrer Besatzung in Stavanger dem neuen Eigentümer, Herrn Simon Mokster, übergeben zu werden. Sie soll dort den Namen „Stril Explorer " bekommen haben. Im Jahr 1991 schied der langjährige Kapitän der "Uthörn", Rudolf Klings, aus dem Dienst der BAH. Sein Nachfolger wurde der Helgoländer HansCarl Lührs. Hans Falke, der Kapitän der "Heincke" und seit 1968 im Dienst der BAH, ging im Dezember 1992 in den Ruhestand.

Im Jahr 1990 übernahm Dipl.-Biol. Hanns-Joachim Neubert das Amt des PR-Sprechers in der Zentrale Hamburg von Dr. Greve. Auf Anregung von Dr. Greve entstand 1991 die "Nathanael-Pringsheim-Gesellschaft zur Förderung der Biologischen Anstalt Helgoland e. V.". Sie soll die Aufgaben der BAH und ihre Mitarbeiter unbürokratisch unterstützen (so wurde als eine der ersten Hilfen eine bessere Absicherung der Studenten und Wissenschaftler auf See ermöglicht), und die Arbeiten an der BAH einem weiteren Personenkreis bekannt und verständlich machen. „Die Gesellschaft trägt den Namen von Nathanael Pringsheim (1823-1894), einem der Hauptinitiatoren zur Gründung der vormals Königlich Biologischen Anstalt Helgoland. Seine Person vereinigte aufs glück- 
lichste in sich den kreativen Wissenschaftler, den großherzigen Mäzen und nicht zuletzt auch den kultivierten Humanisten. Die Leitung der Biologischen Anstalt Helgoland wünscht der Nathanael-Pringsheim-Gesellschaft eine hohe Akzeptanz sowie eine gute Kommunikation mit Menschen sowohl im wissenschaftlichen als auch im außerwissenschaftlichen Feld zum Wohle und zur Steigerung der Leistungsfähigkeit der Biologischen Anstalt Helgoland im zweiten Jahrhundert ihres Bestehens", so sagte Prof. Gunkel in seinem Grußwort am 10.6.1991. Ende 1994 hatte die Pringsheim-Gesellschaft 50 Mitglieder; 1994 wurden 12290 DM ausgegeben mit den größten Posten für PR-Material, Öffentlichkeitsarbeit zur Unterstützung der BAH und Versicherungen von Expeditionsteilnehmern.

1992 feierte die Biologische Anstalt Helgoland ihr 100jähriges Bestehen. Das Festprogramm begann mit einer Ausstellung im Museum Alexander Koenig in Bonn vom 22. 5. bis 19.7. 1992 mit dem Titel "Herausforderung Meer". Studenten des Fachbereichs Gestaltung der Fachhochschule Hamburg der Professoren Flurschütz (Illustrationen) und Putz (Typographie) stellten in 19 farbigen Postern die Arbeitsgebiete und Themen der biologischen Meereskunde dar, in denen die Anstalt tätig ist; von Organismen und deren Funktionen in Ökosystemen und schließlich auch von Schädigungen und deren Ursachen. Ein Aquarium aus fünf Becken brachte das Meeresleben selbst den Besuchern nahe. Der sonst vor Haus C auf Helgoland stehende Meeresglobus, Schollen- und Planktonnet $z$, Dredge, Bagger und Siebe, Taucherausrüstung und Helgoland-Modell sorgten für eine Umrahmung "zum Begreifen ". Der Glückstädter Maler Hans-Peter Wirsing fer-

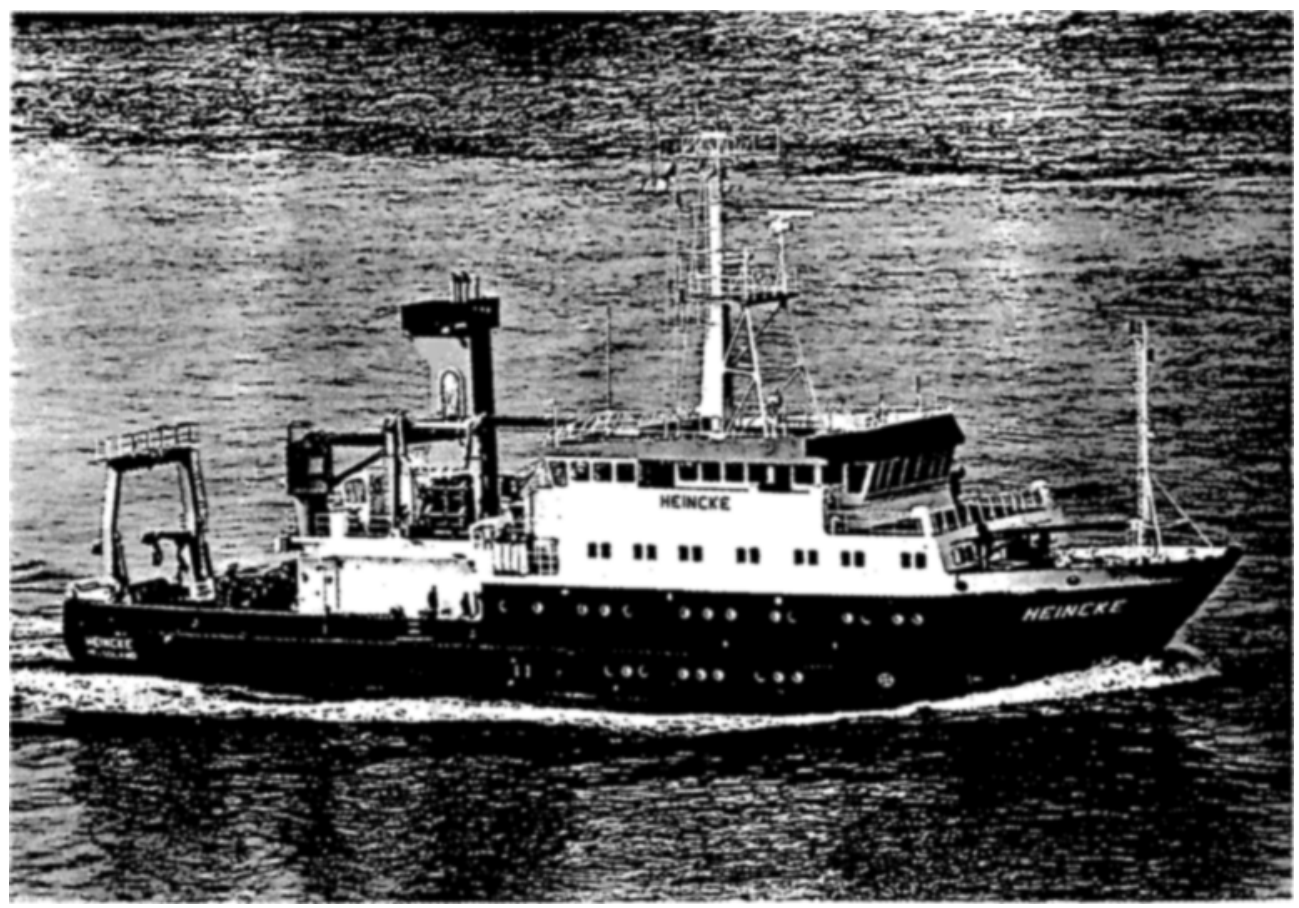

Abb. 43. FS „Heincke“ 1990. Photo: Archiv der BAH. 
tigte das Plakat zur Ausstellung, das zugleich für die Ankündigung des Jubiläumssymposiums auf Helgoland warb (Wiedergabe als Titelfoto dieser Arbeit).

Eine zweite reichhaltige Ausstellung fand vom 1. bis zum 20. September $1992 \mathrm{im} \mathrm{Hel-}$ goländer Rathaus statt mit vielen Bildern, Postern, historischem und neuerem Arbeitsgerät und dem Modell der "Heincke".

Der Höhepunkt des Jubiläums war das wissenschaftliche Symposium vom 13. bis zum 18. September unter dem Motto: "The Challenge to Marine Biology in a Changing World". Auf Helgoland wurde die von der Gemeinde dem Verfall preisgegebene Nordseehalle nach der Prüfung durch das Bauaufsichtsamt durch BAH-Mitarbeiter und Studenten unter der fachmännischen Leitung von Jacob-Karl Holtmann, Olaf Goemann und Dieter Pflaumbaum saniert. In Hamburg wurde das wissenschaftliche Programm durch einen Mitarbeiterstab unter Dr. Siebers und Dr. Sperling vorbereitet.

Zum Symposium kamen dann 450 Teilnehmer aus 36 Ländern. Es stand unter der Schirmherrschaft des Bundespräsidenten Dr. Richard von Weizsäcker; der Bundesminister für Forschung und Technologie, Dr. H. Riesenhuber, und der Planktologe Prof. Karl Banse aus Seattle hielten die Festvorträge. Es folgten, auf $4 \frac{1}{2}$ Tage verteilt, 121 Vorträge, die Vorstellung von 133 Postern, 11 Abendvorträge und Filme, eine Abschlußrunde zum zentralen Thema mit dem Hamburger Botaniker Prof. A. Weber als Leiter, drei gesellige Veranstaltungen, der Besuch von neun Forschungsschiffen, Institutsbesichtigungen und Exkursionen! Die Vorträge füllen den 930 Seiten starken Band 49 der "Helgoländer Meeresuntersuchungen“" (1995).

Organisation und Personal der BAH im Jahr 1992 sind aus Tabelle 4 ersichtlich. Von großer Bedeutung für verschiedene Arbeitsgruppen an der BAH ist die Untersuchung von Enzymen geworden, die sich bei Krebsen und Fischen als entscheidend bei Temperaturanpassung und Biotransformation von organischen Schadstoffen erwiesen haben (Prof. Buchholz, Dr. Angela Köhler-Günther, Dr. v. Westernhagen). In der Abteilung Meeresbotanik hatte Prof. Lüning sein Buch „Meeresbotanik“ bzw. „Seaweeds“ über die Biogeographie und Ökophysiologie der marinen Makroalgen veröffentlicht (Lüning, 1985, 1990), und es gab in den letzten Jahren eingehende Untersuchungen über die Wirkung ultravioletter Strahlung auf Algen aus verschiedenen Tiefen (Dr. M. J. Dring, Prof. Lüning). In Zusammenarbeit mit dem Botanischen Institut und dem Institut für Meereskunde der Universität Hamburg war die Freiland-Kultur von Laminaria-Arten bei Helgoland erfolgreich (Prof. Lüning, Dr. Cornelia Buchholz).

Seit der Stipendiatenzeit an der BAH (1987/88) des heutigen Abteilungsleiters Dr. Christian Schütt gibt es in zunehmendem Maße molekulargenetische Verfahren an der BAH: In Untersuchungen über die Rolle der Plasmide bei umweltrelevanten Aktivitäten der Bakterien, über Endosymbiosen und Interaktionen zwischen Organismen (Viren Prokaryonten - Eukaryonten), und über die Beschreibung von Arten und stammesgeschichtlichen Verwandtschaften. Es wurden in den letzten Jahren u. a. folgende Themen bearbeitet: Die ökologische Bedeutung der Phagen (aufbauend auf den langjährigen Untersuchungen von Dr. Moebus), genetische Anpassung von Bakterienpopulationen an Schwermetalle, Wechselbeziehungen zwischen Noctiluca und Bakterien und die Rolle der Bakterien und Protisten bei der Produktion von Toxinen im Meer. Die molekulare marine Naturstoff-Forschung an der BAH mit der Möglichkeit einer wirtschaftlichen Nutzbarmachung wird vorbereitet.

In der Meeresstation Helgoland begannen im Jahr 1993 Bauarbeiten am Aquarium 
mit umfassender Erneuerung der Becken, da sich durch Seewasser verursachte schwere Schäden an Betonteilen gezeigt hatten. Die Wattenmeerstation Sylt erhielt im Jahr 1996 einen Konferenz- und Seminarraum als Neubau.

Am 5. 8. 1993 wurde dem Kollegium der BAH durch Ministerialdirektor Dr. Mennicken, Abteilungsleiter im Bundesministerium für Forschung und Technologie, mitgeteilt, daß nunmehr der Status der BAH als unselbständige Bundesanstalt definitiv aufgegeben und eine neue Konzeption gefunden werden müsse. Die bisherige Struktur, die sich historisch entwickelt habe, entspräche nicht mehr den Bedürfnissen einer zeitgemäßen Forschungsaktivität. Eine Arbeitsgruppe des Wissenschaftsrates solle unter Einbeziehung des Wissenschaftlichen Beirats der BAH überlegen, wie der Forschungsauftrag der BAH weitergeführt werden könne. Im Frühjahr 1994 wurden die drei BAHStationen durch Mitglieder des Wissenschaftsrates besichtigt, der in seiner "Stellungnahme zur Biologischen Anstalt" vom 20.5. 1994 empfahl, "die Biologische Anstalt Helgoland unter ihrem jetzigen traditionsreichen Namen als ein selbständiges Institut mit zwei Forschungsstandorten auf Helgoland und Sylt weiterzuführen und zu stärken “. Des weiteren heißt es in der Stellungnahme des Wissenschaftsrates: "Die überregionale Bedeutung der BAH und das gesamtstaatliche Interesse an ihrer Forschung und ihren Serviceleistungen auf dem Gebiet der Meeresbiologie stehen nach Ansicht des Wissenschaftsrates außer Frage."

\section{Schlußwort}

Im vorangehenden Kapitel konnte nur sehr wenig der Vielfalt von Arbeiten und Ergebnissen der letzten Jahre untergebracht werden. Die Jahresberichte der BAH zeigen ein ausführlicheres Bild von Personen, Aktivitäten und Arbeitsmitteln. Die Darstellung der Forschungen erfolgt seit 1993 nicht mehr abteilungsweise, sondern nach den Kategorien des Forschungsprogramms. Dieses Programm geht auf den Forschungsplan zurück, den Prof. Gunkel mit den Wissenschaftlern der BAH seit 1990 auf Ersuchen des Ministeriums neu erarbeitet hat. Es soll darin deutlich werden, daß es an der BAH eigenständige Leitlinien für den Forschungsansatz gibt und daß weitgehend interdisziplinär gearbeitet wird. Die Forschungsvorhaben sind in folgenden thematischen Schwerpunkten untergebracht:

- Biologische Dynamik in der Deutschen Bucht,

- Austauschprozesse im Wattenmeer.

- Adaptive Strategien mariner Organismen,

- Taxonomie mariner Organismen,

- Spezielle Projekte.

Die ursprünglich vorgeschlagene Kategorie "Erforschung der Meeresverschmutzung" wurde aufgegeben; die damit befaßten Arbeiten wurden im Zusammenhang mit den übrigen Schwerpunkten gesehen und dort eingeordnet.

In der Fassung des Forschungsprogramms vom Dezember 1991 heißt es in der Präambel:

"Mit diesem Programm verfolgt die BAH eine Doppelstrategie: Das Wissen über die Lebensvorgänge im Meer, besonders in der Nordsee, wird vertieft. Gleichzeitig tragen diese Forschungsarbeiten dazu bei, der Politik vorausschauend geeignete Maßnahmen für den Schutz und die ausgewogene Nutzung des Meeres zu empfehlen." 
So sagte auch Prof. Kinne schon auf dem Symposium zum 75jährigen Bestehen der BAH:

"Wer die Schätze des Meeres nutzt, der muß auch die Bürde der Verantwortlichkeit für dessen Gesunderhaltung tragen. Die Prämissen für die Ausübung dieser Verantwortlichkeit sind wissenschaftliche Erkenntnisse" (Kinne, 1967b).

\section{Literaturverzeichnis}

Anonymus, 1951. Ideen-Wettbewerb für den Wiederaufbau der Insel Helgoland. Kreisverwaltung Pinneberg, 39 pp.

Anonymus, 1957. Bericht der im Dezember 1955 von dem Bundesminister für Ernährung, Landwirtschaft und Forsten eingesetzten Kommission zur Koordinierung der Forschungseinrichtungen seines Geschäftsbereichs. - Manuskript (unpubl.).

Bahr, K., 1962. Rückblick auf die Entwicklung der Deutschen Wissenschaftlichen Kommission für Meeresforschung (DWK) in sechs Jahrzehnten. - Ber. dt. wiss. Kommn Meeresforsch. 17, 34-44.

Biologische Anstalt Helgoland, 1963-1993. Jahresberichte 1962-1992. Boyens, Heide.

Bockwoldt, E. \& Goemann, O., 1988. Helgoland - Eine Arbeitshilfe zum Kennenlernen eines einzigartigen Naturraumes. - Seevögel 9 (Sonderh.), 1-48.

Boley, K., Borchard, T. \& Schrey, E. (Hrsg.), 1992. Ökosystemforschung Wattenmeer. Landesamt für den Nationalpark Schleswig-Holsteinisches Wattenmeer, Tönning. $116 \mathrm{pp}$.

Böhnecke,G. \& Meyl, A.H., 1962. Denkschrift zur Lage der Meeresforschung. Steiner, Wiesbaden, $131 \mathrm{pp}$.

Bückmann, A., 1948. Die Fischerei der Finkenwerder und Schulauer Hochseekutter im Sommer/ Herbst 1945. - Arch. FischWiss. 1, 36-63.

Bückmann, A., 1952. Clemens Künne. - Ber. dt. wiss. Kommn Meeresforsch. 12, 512-515.

Bückmann, A. (Hrsg.), 1959. Die Wiedereröffnung der Biologischen Anstalt Helgoland auf der Insel Helgoland 1959. - Helgoländer wiss. Meeresunters. 7, 1-50.

Bulnheim, H.-P., 1983. Biologische Anstalt Helgoland: Neubau der Zentrale. - GIT Fachz. Lab. 27, 405-414.

Caspers, H., 1938. Die Bodenfauna der Helgoländer Tiefen Rinne. - Helgoländer wiss. Meeresunters. $2,1-162$.

Caspers, H., 1950. Die Lebensgemeinschaft der Helgoländer Austernbank. - Helgoländer wiss. Meeresunters. 3, 119-169.

Courtney, W. A. M. \& Webb, J. E., 1964. The effects of the cold winter of $1962 / 63$ on the Helgoland population of Branchiostoma lanceolatum (Pallas). - Helgoländer wiss. Meeresunters. 10, 301-312.

Drebes, G., 1974. Marines Phytoplankton. Thieme, Stuttgart, 186 pp.

Ehrenbaum, E., 1910. Das Aquarium der Biologischen Anstalt auf Helgoland. - Int. Revue ges. Hydrobiol. Hydrogr. 3, 418-446.

Emschermann, P., Hoffrichter, O., Körner, K. \& Zissler, D. (Hrsg.), 1992. Meeresbiologische Exkursion. Fischer, Stuttgart, $257 \mathrm{pp}$.

Fransz, H. G., Mommaerts, J. P. \& Radach, G., 1991. Ecological modelling of the North Sea. - Neth. J. Sea Res. $28,67-140$.

Geus, A. \& Querner, H., 1990. Deutsche Zoologische Gesellschaft 1890-1990, Dokumentation und Geschichte. Fischer, Stuttgart, 187 pp.

Gillandt, L., 1979. Zur Ökologie der Polychaeten des Helgoländer Felslitorals. - Helgoländer wiss. Meeresunters. 32, 1-35.

Gillbricht, M. 1994. Phytoplankton and the physical environment in the Helgoland region. - Helgoländer Meeresunters. 48, 431-443.

Goemann, O., 1990a. "Echt" Helgoländer Hummer. Kohlrenken, Oldenburg, 88 pp.

Goemann, O., 1990b. Zum Rückgang des Hummerbestandes bei Helgoland. In: Warnsignale aus der Nordsee. Hrsg. von J. L. Lozán, W. Lenz, E. Rachor, B. Watermann \& H. v. Westernhagen. Parey, Berlin, 183-188.

Greve, W., 1968. The "planktonkreisel", a new device for culturing zooplankton. - Mar. Biol. 1, 201-203. 
Hagmeier, A., 1933. Die Züchtung verschiedener wirbelloser Meerestiere. In: Handbuch der Biologischen Arbeitsmethoden. Hrsg. von E. Abderhalden. Urban \& Schwarzenberg, Berlin, Abt. 9, T. 5, 465-598.

Hagmeier, A., 1934. Meeresleben. Aus dem Aquarium der Biologischen Anstalt auf Helgoland. Westermanns Mh. 79, 513-520.

Hagmeier, A., 1951. Notiz über die Biologische Anstalt Helgoland nach dem Kriege, - Zool. Anz. 146, 130-133.

Hagmeier, A., 1952. Helgoland und die Biologische Anstalt. In: Helgoland ruft. Hrsg. von J. Packroß \& P. Rickmers. Schultheis, Hamburg, 9 pp.

Heincke, F., 1894. Die Biologische Anstalt auf Helgoland und ihre Thätigkeit im Jahre 1893. - Wiss. Meeresunters. (Abt. Helgoland) 1, 1-33.

Heincke, E., 1897. Die Thätigkeit der Kgl. Biologischen Anstalt auf Helgoland in den Jahren 1894 bis 1896. - Wiss. Meeresunters. (Abt. Helgoland) 2, 537-579.

Heincke, F., 1918. Die Biologische Anstalt auf Helgoland 1892-1917. - Naturwissenschaften 6 , 569-573.

Henschel, J., 1952. Die Nährstoffgehalte der Miesmuschel (Mytilus edulis) und der Klaffmuschel (Mya arenaria) in ihren jahreszeitlichen Schwankungen. - Helgoländer wiss. Meeresunters. 4, 88-99.

Hertling, H., 1932. Die Züchtung von Meeresfischen für wissenschaftliche und praktische Zwecke. In: Handbuch der Biologischen Arbeitsmethoden. Hrsg. von E. Abderhalden. Urban \& Schwarzenberg, Berlin, Abt. 9, T. 6, 195-366.

Hickel, W., Mangelsdort, P. \& Berg, J. 1993. The human impact in the German Bight: Eutrophication during three decades (1962-1991). - Helgoländer Meeresunters. 47, 243-263.

Holtmann, A., 1967. Seit 65 Jahren ein Schaufenster des Meeres. - Helgoländer 37, 1-3.

Janke, K., 1986. Die Makrofauna und ihre Verteilung im Nordost-Felswatt von Helgoland. Helgoländer Meeresunters. 40, 1-55.

Kesseler, H., 1981. Die Seewasserversorgungsanlage der Litoralstation List/Sylt. - Meerestechnik $12,157-162$.

Kinne, O., 1965. Helgoland und die Meeresbiologie. - Helgoländer 12, 1-4.

Kinne, O., 1967 a. Die Biologische Anstalt Helgoland. Ihre Entstehung und ihre Aufgaben. - Nordfriesland $6,1-14$.

Kinne, O., $1967 \mathrm{~b}$. Die Biologische Anstalt Helgoland. Gegenwart und Zukunft. - Helgoländer wiss. Meeresunters. 16, 419-438.

Kinne, O., 1970. Unterwasserlaboratorium "Helgoland". Ein naturwissenschaftlich-technisches Pionierunternehmen. - Naturwissenschaften 57, 480-487.

Kinne, O., 1980. Die Biologische Anstalt Helgoland. In: Helgoland. Hrsg. von H. P. Rickmers. Dingwort, Hamburg, 75-86.

Kinne, O., 1984. International Helgoland Symposium "Diseases of marine organisms “: Opening address. - Helgoländer Meeresunters. 37, 2-4.

Kinne, O., 1992. Bemerkenswertes aus der jüngeren Geschichte der BAH. - Vortrag, gehalten vor der Nathanael-Pringsheim-Gesellschaft. Manuskript, unpubl.

Kinne, O. \& Rosenthal, H., 1967. Effects of sulfuric water pollutants on fertilization, embryonic development and larvae of the herring, Clupea harengus. - Mar. Biol. 1, 65-83.

Königliche Biologische Anstalt auf Helgoland (Hrsg.), 1914. Tier- und Pflanzenleben der Nordsee. Nach Aquarium-Aufnahmen von F. Schensky. Klinkhardt, Leipzig, 30 Taf.

Kornmann, P., 1952. Die Algenvegetation von List auf Sylt. - Helgoländer wiss. Meeresunters. 4, 55-61.

Kornmann, P. \& Sahling, P.-H., 1977. Meeresalgen von Helgoland. Benthische Grün-, Braun- und Rotalgen. - Helgoländer wiss. Meeresunters. 29, 1-289.

Kremer, B. P. \& Janke, K., 1990. Die Insel Helgoland. Geologische und biologische Anmerkungen zu einem einzigartigen Lebensraum. - Seevögel 11 (Sonderh.) 2, 1-25.

Krumbein, W. E., 1975. Verwitterung, Abtragung und Küstenschutz auf der Insel Helgoland. - Abh. Verh. naturwiss. Ver. Hamburg 18/19, 5-31.

Krumbein, W. E. \& van der Pers, J. N. C., 1974. Diving investigations on biodeterioration by seaurchins in the rocky sublittoral of Helgoland. - Helgoländer wiss. Meeresunters. 26, 1-17. 
Krüß, A., 1988. Die benthische Fischfauna des Helgoländer Felssockels - ein Beitrag zur Biologie und Ökologie der häufigsten Arten. Dipl. Arb., Karlsruhe, 172pp.

Kuckuck, P., 1905. Der Strandwanderer. Lehmanns, München.

Kuckuck, P., 1908. Der Nordseelotse. Meißners, Hamburg, 239 pp.

Kuckuck, P., 1924. Der Nordseelotse. Meißners, Hamburg, 299 pp.

Kuckuck, P., 1974. Der Strandwanderer, 11. Auflage. Hrsg. von J. Gerloff. Lehmanns, München, $264 \mathrm{pp}$.

Kühl, H., 1950. Studien über die Sandklaffmuschel Mya arenaria. - Arch. FischWiss. 2, 25-39.

Kurzweg, C., 1984. BAH. Neubau der Zentrale. - Bauverwaltung 9/84, 376-378.

Lauterborn, R., 1894. Beiträge zur Meeresfauna von Helgoland. V. Die pelagischen Protozoen und Rotatorien Helgolands. - Wiss. Meeresunters. (Abt. Helgoland) 1, 207-213.

Leudesdorff, R., 1987. Wir befreiten Helgoland. Die friedliche Invasion 1950/51. Cobra, Husum, $320 \mathrm{pp}$.

Lippens, P., 1973. Station Helgoland. Müller, Rüschlikon-Zürich, 188 pp.

Lozán, J. L., Lenz, W., Rachor, E., Watermann, B. \& v. Westernhagen, H. (Hrsg.), 1990. Warnsignale aus der Nordsee. Paul Parey, Berlin. $431 \mathrm{pp.}$

Lozán, J. L., Rachor, E., Reise, K., v. Westernhagen, H. \& Lenz, W. (Hrsg.), 1994. Warnsignale aus dem Wattenmeer. Blackwell, Berlin, 387 pp.

Lozán, J. L. \& Kausch, H. (Hrsg.), 1996. Warnsignale aus Flüssen und Ästuaren. Parey, Berlin. 389 pp.

Lozán, J. L., Lampe, R., Matthäus, W., Rachor, E., Rumohr, H. \& v. Westernhagen, H. (Hrsg.), 1996. Warnsignale aus der Ostsee. Parey, Berlin. 385 pp.

Lüning, K., 1985. Meeresbotanik. Verbreitung, Ökophysiologie und Nutzung der marinen Makroalgen. Thieme, Stuttgart, $375 \mathrm{pp}$.

Lüning, K., 1990. Seaweeds. Their environment, biogeography, and ecophysiology. Wiley, New York, $527 \mathrm{pp}$.

Lundbeck, J., 1949. Statistisch-biologischer Bericht über die Anlandungen deutscher Seefischereifahrzeuge an den Fischmärkten Bremerhaven, Cuxhaven und Hamburg-Altona im April 1949. Fischereiwelt 1, 12-13.

Meyer-Waarden, P. F. (Hrsg.), 1962. Festschrift zur Einweihung der Bundesforschungsanstalt für Fischerei. - Arch. FischWiss. 13 (Beih. 1), 1-156.

Mollenhauer, D. \& Lüning, K., 1988. Helgoland und die Erforschung der marinen Benthosalgen. Helgoländer Meeresunters. 42, 385-425.

Niermann, U., 1997. Macrobenthos of the south-eastern North Sea during 1983-1988. - Ber. Biol. Anst. Helgoland 13,1-144

Nies, H., Jenke, H.-S. \& Sperling, K.-R. 1994. Das Laboratorium Sülldorf - Entwicklung und Chancen. - Mitt. dt. Ges. Meeresforsch. 1/1994, 23-26.

Peters, G. (Hrsg.), 1984. Karl Ritter von Frisch-Medaille. Wissenschaftspreis 1984 der Deutschen Zoologischen Gesellschaft. - Verh. dt. Zool. Ges. 77 (Beih.).

Radach, G. \& Berg, J., 1986. Trends in den Konzentrationen der Nährstoffe und des Phytoplanktons in der Helgoländer Bucht (Helgoland Reede Daten). - Ber. Biol. Anst. Helgoland 2, 1-63.

Ramster, J., 1975. Dr. J. N. Carruthers, 24 November 1885 - 8 March 1973. - J. Cons. int. Explor. Mer $36,101-105$.

Reise, K., 1985. Tidal Flat Ecology. An experimental approach to species interactions. Springer Berlin, $191 \mathrm{pp}$.

Renckhoff, I., 1992. Die "Bio“ und ihre große Bibliothek. - Helgoländer 393, 23-24.

Scherffel, A., 1899. Phaeocystis globosa n. sp. (Vorl. Mitt.). - Ber. dt. bot. Ges. 17, 317-318.

Schlieper, K. (Hrsg.), 1968. Methoden der meeresbiologischen Forschung. Fischer, Jena, 322 pp.

Schmidt, U., 1970. Professor Dr. Adolf Bückmann zum siebzigsten Geburtstag. - Ber. dt. wiss. Kommn Meeresforsch. 21, 3-15.

Schreiber, E., 1927. Die Reinkultur von marinem Phytoplankton und deren Bedeutung für die Erforschung der Produktionsfähigkeit des Meerwassers. - Wiss. Meeresunters. (Abt. Helgoland) 16 , $1-34$.

Tesch, F.-W., 1973. Der Aal. Biologie und Fischerei. Parey, Hamburg, 306 pp. 
Tesch, F.-W., 1977. The eel. Biology and management of anguillid eels. Chapman \& Hall, London, $434 \mathrm{pp}$

Tesch, F.-W., 1983. Der Aal. Biologie und Fischerei. Parey, Hamburg, 340 pp.

Timm, R., 1894. Beiträge zur Fauna der südöstlichen und östlichen Nordsee. IV. Copepoden und Cladoceren. - Wiss. Meeresunters. (Abt. Helgoland) 1, 363-402.

Uhlig, G., 1966. Tauchgruppe im Dienste meeresbiologischer Forschung. - Helgoländer 28, 1.

Uhlig, G., 1971. Ersterfahrungen mit dem UWL „Helgoland“. - Dräger Tauchtechnik 5, 35-45.

Vauk, G., 1977. Geschichte der Vogelwarte und der Vogelforschung auf Helgoland. Niederelbe Druck, Otterndorf, $160 \mathrm{pp}$.

Werner, P., 1993. Die Gründung der Königlichen Biologischen Anstalt auf Helgoland und ihre Geschichte bis 1945. - Helgoländer Meeresunters. 47 (Suppl.), 1-182.

Westheide, W., 1966. Zur Polychaetenfauna des Eulitorals der Nordseeinsel Sylt. - Helgoländer wiss. Meeresunters. 13, 203-209. 


\section{Tabellenanhang}

Tabelle 1. BAH 1964, Organisation und Personal

Leitung: Direktor: Prof. Dr. Otto Kinne; Stellvertreter: Dr. Horst Aurich

Abteilung Zoologie: Prof. Dr. Otto Kinne (Leiter), Dr. Bernhard Werner, Dr. Erich Ziegelmeier, Dr. Gotram Uhlig

Abteilung Physiologie: Prof. Dr. Friedrich Krüger (Leiter), Dr. Hans-Peter Bulnheim, Dr. Heinz Schaefer

Abteilung Botanik: Dr. Peter Kornmann (Leiter), Dr. Hanswerner Kesseler

Abteilung Mikrobiologie: Dr. Wilfried Gunkel (Leiter)

Abteilung Planktologie: Dr. Horst Aurich (Leiter), Dr. Max Gillbricht, Dr. Erik Hagmeier

Abteilung Ichthyologie: Dr. Adolf Kotthaus (Leiter), Dr. Jürgen Flüchter, Dipl-Biol. Harro Haß (Bordbiologe)

Abteilung Strahlenbiologie: Dr. Horst Aurich (Leiter), Dr. Manfred Hoppenheit

Verwaltung: Erich Windt (Leiter), Agnes Heinemann, Ingeborg Heikenwälder, Agnes Libor, Gertrud Wurl (Direktor-Vorzimmer)

Aquarium: Andreas Holtmann (Leiter), Henry Dörner, Jack Friedrichs. Hinrich Kanje

Materialversand: Heinrich Herzog (Leiter), Elke Giersch, Inge Schmolke

Bibliothek: Dipl.-Bibl. Werner Meiss

Technische Assistenten und Laboranten: Francis Crouse, Helmut Eggers, Olaf Goemann, ErichHartwig Harms, Jacob Karl Holtmann. Johann Kahlcke, H. Krohn, Eva-Maria Krüss, Jürgen Marschall, Margarete Mühlenkamp, P. Pflaum, Gerrit Sabling, Paul-Heinz Sahling, Hanna Schmidt, He. Schmidt, Martin Söhl, Hans-Hermann Trekel

Schiffspersonal: Tönnies Hornsmann (Kapitän der „Uthörn“), John Herzog (Schiffsführer der „Ellenbogen“), Werner Krühs, Peter Singer, Jürgen Stoldt, Nickels Wichers, Günter Wolf

Fahrer: Hans Wiegleb

Werkstätten: Charles Lührs, Max Simonsen

Hauspersonal: L. Bredau, Emil Classen, Petrine Gosda, Johanna Klaar, Erna Ralfs, Franz Ralfs, Dorothea Ried

Tabelle 2. BAH 1972, Organisation und Personal

Leitung: Direktor: Prof. Dr. Otto Kinne, Stellvertreter: Prof. Dr. Adolf Kotthaus

Abteilung Meereszoologie: Dr. Hans-Peter Bulnheim (Leiter), Dr. Gerhard Lauckner, Dr. Gustav-Adolf Paffenhöfer, Dr. Dietrich Siebers, Dr. Friedrich-Wilhelm Tesch, Dr. Bernhard Werner, Dr. Erich Ziegelmeier

Abteilung Meeresbotanik: Dr. Dr. h.c. Peter Kornmann (Leiter), Dr. Gerhard Drebes, Dr. Hanswerner Kesseler, Dr. Klaus Lüning

Abteilung Biologische Ozeanographie: Prof. Dr. Adolf Kotthaus (Leiter), Dr. Erik Hagmeier, Dr. Wolfgang Hickel 
Tabelle 2. (Fortsetzung)

\begin{abstract}
Abteilung Experimentelle Ökologie: Dr. Gotram Uhlig (Leiter), Dr. Jürgen Flüchter, Dr. Wulf Greve, Dr. Manfred Hoppenheit, Dr. Hermann Kayser, Dr. Harald Rosenthal, Dr. Klaus-Richard Sperling
\end{abstract}

Abteilung Meeresmikrobiologie: Dr. Wilfried Gunkel (Leiter), Dr. Wolfgang Krumbein, Dr. Karlheinz Moebus

Taxonomische Arbeitsgruppe: Dipl.-Biol. Hans Georg Andres, Dr. Ilse Bartsch, cand.rer.nat. K.-H. Becker, Dipl.-Biol. Ingo Freudenhammer, Dr. habil. Gesa Hartmann-Schröder, Dipl.-Biol. Helga Kapp, Dr. Anton Lelek, Dr. Karin Riemann-Zürneck, Dr. Hartmut Schinkowski, Dr. Renate Weigmann-Haass

Emeriti: Dr. Horst Aurich, Prof. Dr. Friedrich Krüger

Doktoranden: Peter Jatzke, Dipl.-Biol. Karl Klöckner, Gertraud Luther, J. Raff

Verwaltung: Uwe Kersten (Leiter), Ilse Gätjens, Agnes Heinemann, Agnes Libor, Anneliese Lorenz, Marie-Louise Schröder, Gertrud Wurl (Direktor-Vorzimmer)

Aquarium: Andreas Holtmann (Leiter), Josef Baumann, Helgo Block, Nikolaus Loch, Carl Rickmers

Materialversand: Heinrich Herzog (Leiter), Gerda Duve, Olaf Goemann

Bibliothek: Dipl.-Bibl. Inka Schritt, Dipl.-Bibl. Beate Sysoew, Johanna Klaar

Technische Assistenten und Laboranten: I. Bacvarov, Marianne Bartel, Rainer Benesch, Valerie Clark, Werner Dittrich, Brigitte Freier, Gudrun Fürstenberg, Marianne Greve, Elisabeth Grönstad, Hannelore Harbst, Erich-Hartwig Harms, Sieglinde Hogreve, Jacob K. Holtmann, Petra Hosumbek, Jutta Klinckmann, Karl-Walter Klings, Margot Koschorrek, Käthe Krüss, R. Kruse, D. Lemcke, Rainer Lüdtke, Jürgen Marschall, Dorothed Martin, B. Metscher, U. Michels, Alfred Mielke, Sybille Mielke, Margarete Mühlenkamp, K. Neubauer, Sigrid Ohlsen, H. Rade, A. Reiners, Inge Reyelts, Ute Rieper, Gerrit Sahling, Paul-Heinz Sahling, Martin Söhl, Manfred Sturm, Günther Tadday, Klaus Treutner, A. Winter, Helga Witt.

Schiffspersonal: Hans Falke, James Friedrichs, Jens-Uwe Helmke, Tönnies Hornsmann (Kapitän der "Friedrich Heincke"), John Hottendorf, Klaus Ivens, Gerhard Jung, Rudolf Klings, Gerd Krüss, Werner Krühs (Schiffsführer der „Ellenbogen“), Adolf Kruse, W. Leiser, Hans Ernst Lorenzen, G. Mensing, Volker Oelrichs, G. Scholz, Wolfgang Springer, Jürgen Stoldt (Kapitän der "Uthörn"), R. Thoede, Henry Tönnies, Sibo Wessels, Nickels Wichers, Günter Wolf

Tauchergruppe: Werner Schomburg (Leiter), Hans Belau, Dipl.-Ing. Günther Luther, N. Neukirch, Manfred Pose

Fahrer: Erich Koal

Werkstätten: Ernst Ahrens, Richard Gebhardt, H. Handrek, Bernhard Ipsen, Charles Lührs, Erk Lützen, Jürgen Meyer, Martin Wichers

Hauspersonal: Inge Ahrens (Heimleiterin "Arthur-Hagmeier-Haus “), Erika Asmussen (Heimleiterin "Adolf-Bückmann-Haus"), Petrine Gosda, Heidemarie Ipsen, M. Jacob, Bärbel Pflaumbaum, Dieter Pflaumbaum (Heimleiter „Wilhelm-Mielck-Haus“), Erna Ralfs, Franz Ralfs, Dorothea Ried 
Tabelle 3. BAH 1982, Organisation und Personal (auszugsweise)

Leitung: Direktor: Prof. Dr. Otto Kinne; Stellvertreter: Prof. Dr. Hans-Peter Bulnheim

Abteilung Meereszoologie: Prof. Dr. Hans-Peter Bulnheim (Leiter), Dr. Klaus Anger, Dr. Gerhard Lauckner, Dr. Dietrich Siebers, Dr. Friedrich-Wilheim Tesch, Dipl.-Biol. Ruth Böttger (bis 31. 8.), Dipl.-Biol, Rüdiger Kracht (bis 31.8.), Dr. Hein von Westernhagen, Dipl.-Biol. Monika Schoth, Dipl.-Biol. Andreas Winkler, Dipl.-Biol. Ulrich Niermann

Abteilung Meeresbotanik: Dr. Klaus Lüning (Leiter), Dr. Gerhard Drebes, Dr. Erik Hagmeier, Dr. Hanswerner Kesseler.

Abteilung Biologische Ozeanographie: Prof. Dr. Max Gillbricht (Leiter), Dr. Wolfgang Hickel, Dr. Peter Martens

Abteilung Experimentelle Ökologie: Dr. Gotram Uhlig (Leiter), Dr. Wulf Greve, Dr. Manfred Hoppenheit, Dr. Volker Kartheus (bis 30.6.), Dr. Hermann Kayser, Dr. Karl Klöckner, Dr. Harald Rosenthal, Dr. Klaus-Richard Sperling, Dr. Ellen Wahl, Dipl.-Biol. Andreas Dietrich (bis 30.6.), Elke Hilpert (bis 30.6.), Dipl.-Biol. Norbert Laasch (bis 30.6.)

Abteilung Meeresmikrobiologie: Dr. Wilfried Gunkel (Leiter), Dr. Günter Gassmann, Dr. Karlheinz Moebus، Dr. Marianna Rieper

Taxonomische Arbeitsgruppe: Dr. Kunigunde Hülsemann (Leiterin), Dr. Hans Georg Andres, Dr. Ilse Bartsch, Dr. Malte Elbrächter, Dr. Renate Haaß, Dr. Gesa Hartmann-Schröder, Dr. HansChristian John, Dipl.-Biol. Helga Kapp, Dr. Karin Riemann-Zürneck, Dr. Knud Schulz, Dr. Christine Karrer (ab 1.5.)

Verwaltung: Uwe Kersten (Leiter)

Direktor-Vorzimmer: Rosemarie Weidel

Aquarium und Materialversand: Olaf Goemann (Leiter)

Bibliothek: Dipl,-Bibl. Inka Schritt (Leiterin), Dipl,-Bibl. Beate Sysoew, Johanna Klaar, Hartmut Hasselberg

Schiffsführung: Hans Falke (Kapitän der „Friedrich Heincke“), Rudolf Klings (Kapitän der "Uthörn")

Tauchgruppenleiter: Werner Schomburg

Hauspersonal (Heimleitung): Waltraud Lorenzen (Heimleiterin „Arthur-Hagmeier-Haus“), Dieter Pflaumbaum (Heimleiter "Wilhelm Mielck-Haus"), Ingrid Weise (Heimleiterin "Adolf-Bückmann-Haus")

Tabelle 4. BAH 1992, Organisation und Personal

Leitung: Prof. Dr. Wilfried Gunkel; Vertreter: Dr. Hein v. Westernhagen (bis 30.9.), Dr. Manfred Hoppenheit (ab 1.10.)

Abteilung Meereszoologie: Prof. Dr. Friedrich Buchholz (ab 1.4., Leiter), Dr. Klaus Anger, Dr. Joachim Harms (ab 15.4.), Dr. Angela Köhler-Günther, Dr. Gerhard Lauckner, Dr. Dietrich Siebers, Dr. Hein von Westernhagen. Auf Projektstellen: Dr. Kirsten Böttcher (DFG), Dipl.-Biol. Patricia Cameron (UBA), Dipl.-Biol. Sven Riestenpatt (ab 1.7., DFG)

Abteilung Meeresbotanik: Prof. Dr. Klaus Lüning (Leiter), Dr. Inka Bartsch, Dr. Gerhard Drebes. Auf Projektstellen: Dipl.-Biol. Petra Leukart, Dipl.-Biol. Britta Schaffelke 
Tabelle 4. (Fortsetzung)

Abteilung Biologische Ozeanographie: Prof. Dr. Karsten Reise (Leiter), Dr. Werner Armonies, Dr. Harald Asmus, Dr, Ragnhild Asmus, Dr. Erik Hagmeier, Dr. Wolfgang Hickel, Dr. Peter Martens. Auf Projektstellen: Dipl.-Biol. Annelise Albrecht (BMFT), Joachim Berg (Umwelt-Bundesamt Berlin, UBA), Dipl.-Biol. Insa Bock (bis 31.7., BMFT), Dipl.-Biol. Frank Buhs (ab 1. 2., Landesamt für den Nationalpark Schleswig-Holsteinisches Wattenmeer, Tönning, NPA), Dipl.-Biol. Ute Kopacz (BMFT), Dr. Dagmar Lackschewitz (ab 1.2.), Dipl.-Biol. Erwin Perlich (BMFT), Dr. Jens Prena (bis 31. 1., UBA), Dipl.-Chem. Thomas Raabe (UBA), Dr. Gerald Schneider (BMFT), Dipl.-Biol. Dirk Schories (BMFT), Dipl--Biol. Ina Siebert (UBA), Dipl.-Biol. Michael Simon (ab 1. 2., NPA), Dipl.-Biol. Martin Thiel (ab 1.10., NPA), Dipl.-Biol. Anke Wille (BMFT)

Abteilung Experimentelle Ökologie: Dr. Gotram Uhlig (bis 30.4., Leiter), Dr. Heinz-Dieter Franke, Dr. Wulf Greve, Dr. Manfred Hoppenheit, Dr. Klaus-Richard Sperling, Dr. Ellen Wahl

Abteilung Meeresmikrobiologie: Dr. Christian Schütt (ab 1.7., Leiter), Dr. Günter Gassmann, Dr. Marianna Kirchner, Dr. Karlheinz Moebus. Auf einer Projektstelle: Dr. Dietmar Glindemann (bis 31.5.)

Taxonomische Arbeitsgruppe: Dr. Hans Georg Andres (Leiter), Dr. Ilse Bartsch, Dr. Malte Elbrächter, Dr. Renate Haass, Dr. Gesa Hartmann-Schröder, Dr. Kunigunde Hülsemann (bis 31. 10.), Dr. Hans-Christian John, Dipl.-Biol. Helga Kapp, Dr. Karin Riemann-Zürneck, Dr. Knud Schulz. Auf einer Projektstelle: Dipl.-Biol. Clementine Zelck

Verwaltung: Uwe Kersten (Leiter), Gerda Bardt, Marie-Luise Bertram, Renate Bötjer, Gerda Duve, Maike Gläsener, Ursel Heitmann, Gertraude Hennemann, Manfred Jung, Veronika Kaufmann, Petra Mönch, Ralf Napirata, Jörg Palenczat, Brigitte Rauch, Renate Schenk, Elke Schmidt, Horst Schröder, Cornelia Seidel, Rosemarie Weidel (Direktor-Vorzimmer), Gaby Zawal

Pressesprecher: Dipl.-Biol. Hanns-Joachim Neubert

Aquarium und Materialversand: Olaf Goemann (Leiter), Ulrich Alexander, Helgo Block, Franz Hennemann, Peter Klose, Alfred Mielke

Bibliothek: Dipl.Bibl. Inka Renckhoff (Leiterin), Ute Alexander, Werner Sisolewsky, Stephani Soll, Dipl.-Bibl. Beate Sysoew

Technische Assistenten und Laboranten: Sieglinde Bahns, Barbara Bahr, Carol Berger, Gertrud Delfs, Brigitte Dethlefs, Gudrun Fürstenberg, Hannelore Halliger, Erich-Hartwig Harms, Elisabeth Herre, Nicole Hildebrandt, Jacob Karl Holtmann, Michael Janke, Dietrich Janssen, Petra Kadel, Renate Kleinfeld, Karl-Walter Klings, Günter Krüner, Bjarne Lauritzen, Peter Mangelsdorf, Cornelia Püschel, Hans Reichenberger, Frank Reiners, Gerrit Sahling, Franziska Schorn, Manfred Sturm, Ute Süsens, Günther Tadday, Klaus Treutner, Ulrike Wiege, Jutta Willführ-Nast

Schiffspersonal: Klaus Bartsch, Peter Botter, Peter Elvert, Hans Falke (Kapitän der "Heincke“), Ernst-Dieter Hansen, Klaus Ivens, Dieter Klings (Schiffsführer der "Diker“), Niels Kruse (Schiffsführer der "Mya”), Gerhard Krüss, Hans-Carl Lührs (Kapitän der "Uthörn“), Lajos Miszler, Hermann Mohr, Volker Oelrichs, Walter Röw (Schiffsführer der "Aade“), Kurt Schmidt, Henry Tönnies, Niels Tönnies, Björn Venske, Robert Voss

Tauchergruppe: Udo Schilling (Leiter), Hans-Hermann Lösche

Fahrer: Hans Geisler

Werkstätten: Karl-Heinz Boysen, Helgo Denker, Karl Gigla, Bernhard Ipsen, Hans-Jürgen Lorenzen, Erk Lützen, Reimer Magens, Manfred Pieper, Walter Reck, Martin Schelp

Hauspersonal: Erika Broders, Waltraut Lorenzen (Heimleiterin "Arthur-Hagmeier-I Taus"), Werner Lorenzen, Cornelia Mumm, Bärbel Pflaumbaum, Dieter Pflaumbaum (Heimleiter „WilhelmMielck-Haus“), Johann Piorek, Regina Piorek, Christiana Reichenberger, Gudrun Schulze, Gabriele Thedens, Ingrid Weise (Heimleiterin "Adolf-Bückmann-Haus") 
Tabelle 5. Kurs der BAH für Biologielehrer 1950 (Ellenbogen/Sylt)

Do. 13. 7 .

Einführung (Prof. Hagmeier)

Dredgefahrt Austernbank, Dredgefahrt vor List, Demonstration des gefischten

Materials (Dr. Werner, Dr. Ziegelmeier, Dr. Kornmann)

Fr. 14. 7 .

Strandwanderung (Dr. Kornmann, Dr. Werner, Dr. Ziegelmeier)

Mollusken, künstliche Befruchtung bei Sabellaria, Barnea candida (Dr. Werner)

Sa. 15. 7 .

Wattexkursion, Demonstration (Dr. Kornmann, Dr. Werner, Dr. Ziegelmeier)

So. 16. 7 .

Inselexkursion, Vogelkoje, Rotes Kliff, Keitum, Morsumkliff, Rantumbecken

(Dr. Kornmann, Dr. Werner)

Mo. 17. 7 .

Algenexkursion, Algenauflegen, Algenmikroskopie (Dr. Kornmann)

Di. 18.7.

Plankton, Hydrographie (Dr. Aurich)

Mi. 19. 7 .

Krebse (Dr. Ziegelmeier)

Echinodermen, künstliche Befruchtung (Dr. Aurich)

Do. 20. 7.

Landexkursion (Dr. Kornmann)

Muschelbank (Dr. Ziegelmeier)

Plankton (Dr. Aurich)

Fr. 21. 7.

Ausfahrt mit der "Uthörn" (Dr. Lundbeck)

Sa. 22.7.

Fischereiwissenschaft (Dr. Lundbeck)

Mo. 24. 7 .

Coelenteraten, Muschelfischerei (Dr. Werner)

Di. 25. 7.

Plankton (Dr. Aurich)

Kleinere Tiergruppen (Dr. Werner)

Ökologie, Lebensweise der Wattenmeertierwelt (Prof. Hagmeier) 
Tabelle 6. Meeresbiologischer Kurs der BAH 1964 (Helgoland) - NW = Niedrigwasser

Di. 4. 8. NW 14.40

8.30 Begrüßung. Grundsätzliches zur Problematik der marinen Biologie (Prof. Kinne)

9.30 Kurze Einführung in die Geologie Helgolands (Dr. Werner), Inselrundgang gemeinsam mit Dr. Kesseler (Landpflanzen) und Dr. Vauk (Vogelwelt)

14.30-18.00 Planktonuntersuchungen. Einführung in die marine Planktonkunde. Übersicht über die wichtigsten Formen anhand von lebendem Material (Dr. Aurich, Dr. Gillbricht)

Mi. 5. 8. NW 15.59

8.30-12.00 Dünenexkursion, Strandwanderung, Untersuchung der Sandlückenfauna (Dr. Uhlig, Dr. Werner, Dr. Ziegelmeier)

14.30-18.00 Bearbeitung des Meiobenthosmaterials der Dünenexkursion. Aufgabe und Bedeutung der marinen Protozoologie. Demonstration einiger marinen Protozooentypen (Dr. Uhlig)

Do. 6. 8. NW 17.08

8.00 Ausfahrt mit F.K. "Uthörn“: Hydrographie, Plankton-, Dredsch- und Bodengreiferfänge, Grundschleppnetzzug

15.00-18.00 Demonstration des gesammelten Materials, Lebendbeobachtungen (Dr. Ziegelmeier)

Fr. 7. 8. NW 18.08

8.30-12.00 Seeigelentwicklung nach künstlicher Befruchtung bei Psammechinus miliaris (Dr. Werner, Dr. Ziegelmeier, A. Holtmann)

14.30-18.00 Beobachtungen und physiologische Versuche an lebendem Material (Prof. Schlieper)

Sa. 8. 8. NW 6.30

8.30-12.00 Fischbiologie, Präparationsübungen an Fischen (Dr. Kotthaus)

Mo. 10.8. NW 8.06

7.00 Exkursion zurn Nordost-Felswatt (Dr. Werner, Dr. Ziegelmeier)

14.30-16.00 Demonstration des gesammelten Materials (Dr. Werner, Dr. Ziegelmeier)

16.00-18.00 Lebendbeobachtungen und physiogische Versuche an marinen Wirbellosen (Prof. Schlieper)

Di. 11. 8. NW 8.48

7.30 Algenexkursion, anschließend Anlegen eines Algenherbars. Mikroskopische Übungen zur Morphologie und Entwicklung von Meeresalgen (Dr. Kornmann, Dr. Kesseler, P. H. Sahling)

Mi. 12. 8. NW 9.26

8.30- 9.45 Stoffwechselphysiologische Probleme in der marinen Botanik (Dr. Kesseler)

10.00-12.00 Bestimmungsübungen an Muscheln (Dr. Ziegelmeier)

nachmittags frei für selbständiges Aufarbeiten des gesammelten Materiais

Do. 13.8. NW 10.02

8.30-12.00 Vorkommen, Verbreitung und Aktivitäten von marinen Mikroorganismen, mit Demonstrationen (Dr. Gunkel)

14.30-18.00 Planktonuntersuchungen, Studium pelagischer Entwicklungsstadien (Dr. Aurich, Dr. Gillbricht)

Fr. 14. 8. NW 10.38

8.30-12.00 Präparation von Arenicola marina, Demonstration zur Biologie von Arenicola (Prof. Krüger)

14.30-18.00 Einführung in die Arbeiten der Biologischen Anstalt Helgoland. Rundgang durch Meeresstation und Aquarium (Prof. Kinne)

Sa. 15. 8. NW 11.17

8.30-11.15 Einrichtung von Seewasseraquarien, Haltung von Meerestieren (A. Holtmann) 11.30 Schlußbesprechung (Prof. Kinne)

Vorgesehen ist eine Besichtigung der Vogelwarte Helgoland, Termin nach Vereinbarung 